\title{
A Bioinspired Cyclization Sequence Enables the Asymmetric Total Synthesis of Dictyoxetane
}

\author{
Cedric L. Hugelshofer and Thomas Magauer \\ Department of Chemistry and Pharmacy, Ludwig-Maximilians-University Munich, \\ Butenandtstrasse 5-13, 81377, Munich, Germany
}

\section{Supporting Information}




\section{Table of Contents}

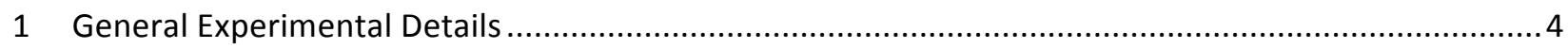

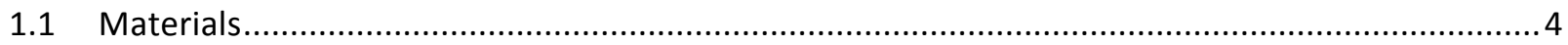

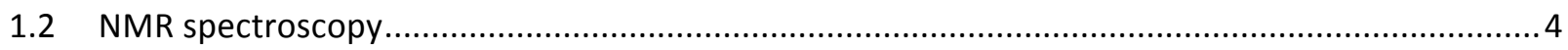

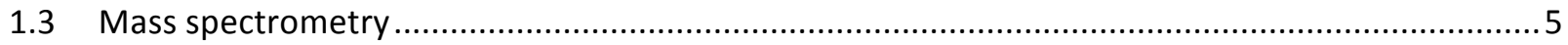

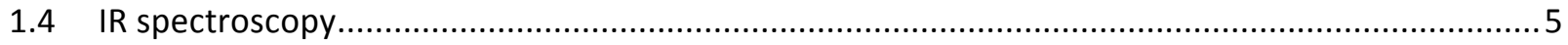

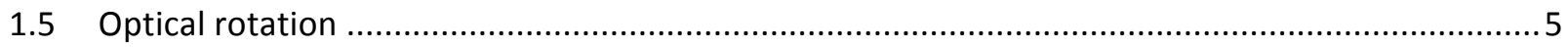

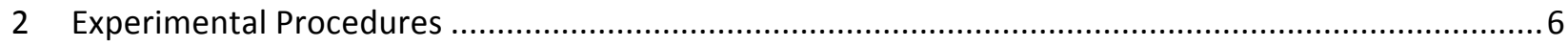

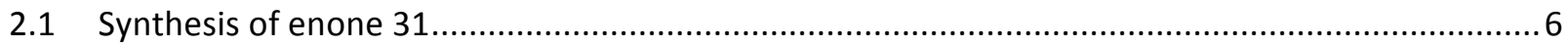

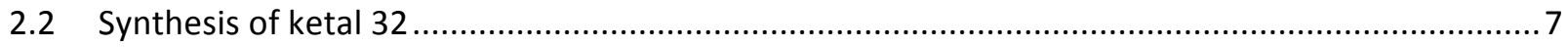

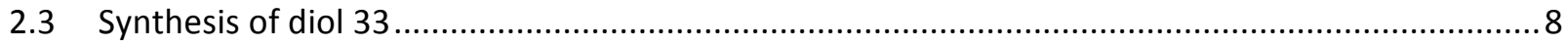

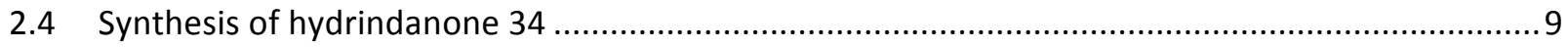

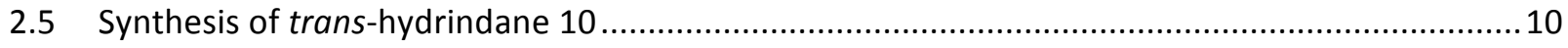

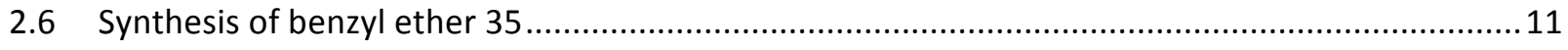

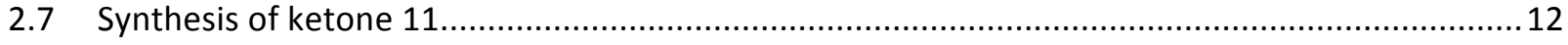

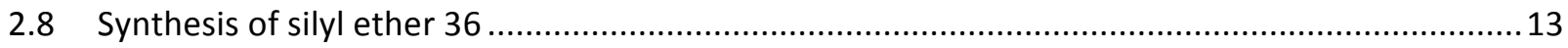

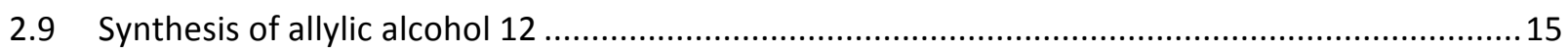

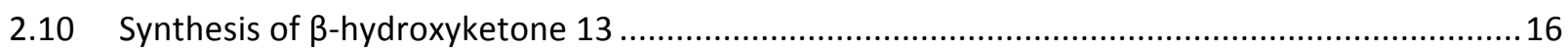

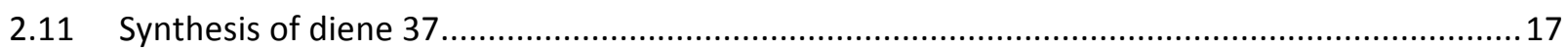

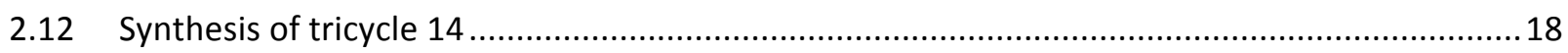

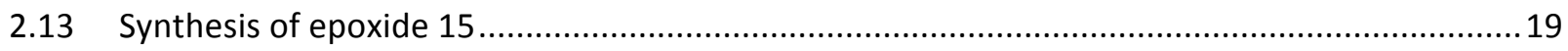

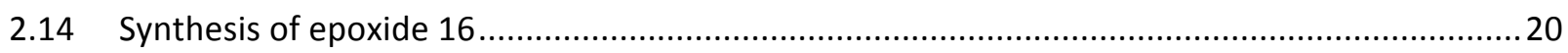

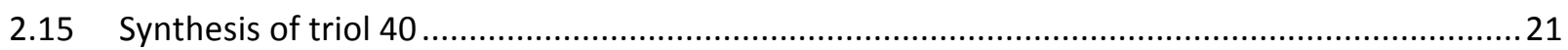

2.16 Synthesis of $1,10,13,14$-tetra-epi dictyoxetane (tetra-epi-1) ................................................2

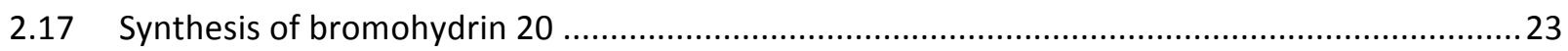

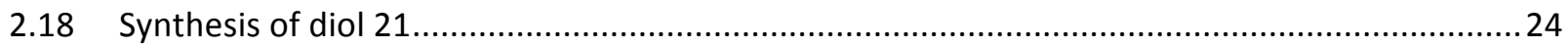

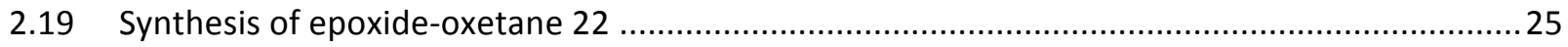

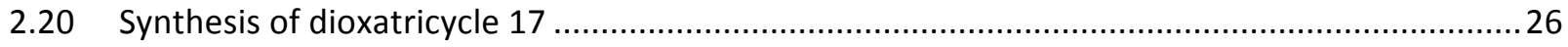

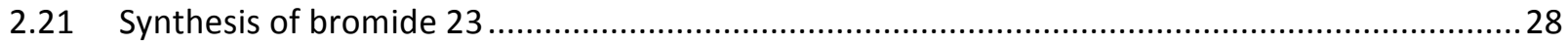

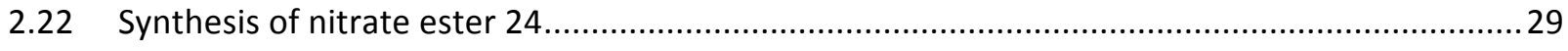

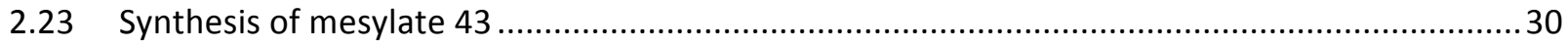

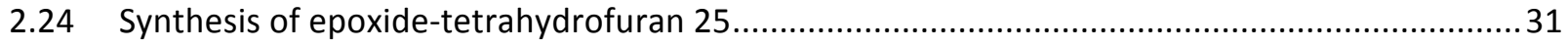

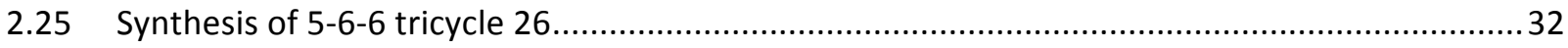

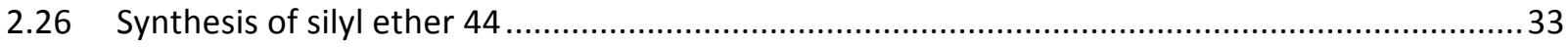

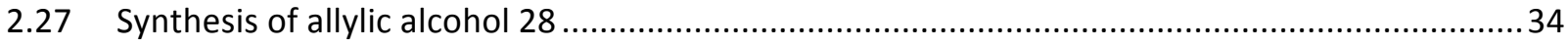




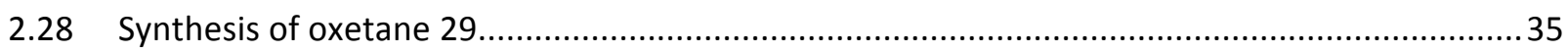

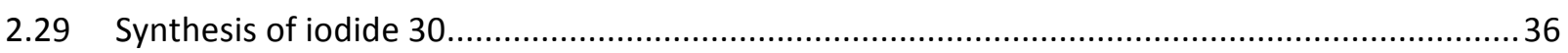

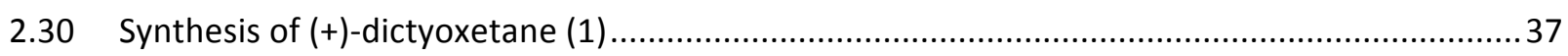

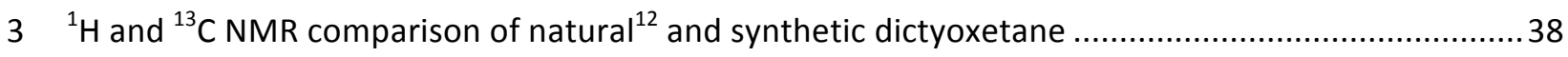

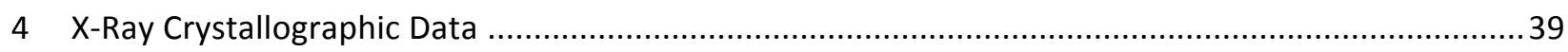

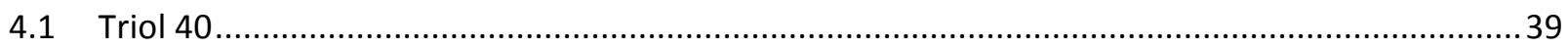

4.2 1,10,13,14-tetra-epi dictyoxetane (tetra-epi-1) ................................................................ 40

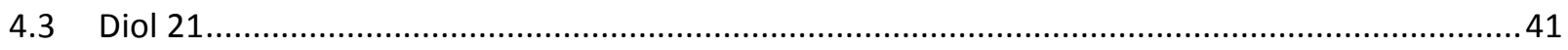

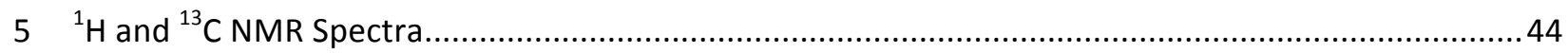




\section{General Experimental Details}

All reactions were performed in flame-dried glassware fitted with rubber septa under a positive pressure of argon, unless otherwise noted. Air- and moisture-sensitive liquids were transferred via syringe or stainless steel cannula through rubber septa. Solids were added under inert gas or were dissolved in appropriate solvents. Low temperature-reactions were carried out in a Dewar vessel filled with a cooling agent: acetone/dry ice $\left(-78{ }^{\circ} \mathrm{C}\right)$, $\mathrm{H}_{2} \mathrm{O} /$ ice $\left(0{ }^{\circ} \mathrm{C}\right)$. Reaction temperatures above $23{ }^{\circ} \mathrm{C}$ were conducted in a heated oil bath. The reactions were magnetically stirred and monitored by NMR spectroscopy or analytical thin-layer chromatography (TLC), using glass plates precoated with silica gel $(0.25 \mathrm{~mm}, 60-\AA ̊ \AA$ pore size, Merck) impregnated with a fluorescent indicator (254 $\mathrm{nm}$ ). TLC plates were visualized by exposure to ultraviolet light (UV), were stained by submersion in aqueous potassium permanganate solution $\left(\mathrm{KMnO}_{4}\right)$ or ceric ammonium molybdate solution (CAM), and were developed by heating with a heat gun. Flash-column chromatography on silica gel was performed as described by Still et al., ${ }^{1}$ employing silica gel ( $60 \AA, 40-63 \mu \mathrm{m}$, Merck KGaA). Flash-column chromatography on silica gel using triethylamine pretreated silica gel was performed by preparing the silica gel slurry with triethylamine $(10 \% \mathrm{v} / \mathrm{v}$ in corresponding eluent mixture) and flushing the column with the eluent prior to loading the compound on the column. The yields refer to chromatographically and spectroscopically $\left({ }^{1} \mathrm{H}\right.$ and $\left.{ }^{13} \mathrm{C} N \mathrm{NMR}\right)$ pure material.

\subsection{Materials}

Tetrahydrofuran (THF) and diethyl ether $\left(\mathrm{Et}_{2} \mathrm{O}\right)$ were distilled from $\mathrm{Na} /$ benzophenone prior to use. Dichloromethane $\left(\mathrm{CH}_{2} \mathrm{Cl}_{2}\right)$, triethylamine $\left(\mathrm{Et}_{3} \mathrm{~N}\right)$, diisopropylamine (DIPA) and $\mathrm{N}, \mathrm{N}$-diisopropylethylamine (DIPEA) were distilled under nitrogen atmosphere from $\mathrm{CaH}_{2}$ prior to use. Benzene, dimethylformamide (DMF), dimethylacetamide (DMA), dimethyl sulfoxide (DMSO), acetonitrile (MeCN), toluene and methanol $(\mathrm{MeOH})$ were purchased from Acros Organics as 'extra dry' reagents and used as received. All other reagents and solvents were purchased from chemical suppliers (Sigma-Aldrich, Acros Organics, Alfa Aesar, Strem Chemicals, ABCR) and were used as received. Solvents for extraction, crystallization and flash-column chromatography on silica gel were purchased in technical grade and distilled under reduced pressure prior to use. $4 \AA$ molecular sieves were washed (methanol, acetone, dichloromethane) and then dried at $100{ }^{\circ} \mathrm{C}$ under vacuum $(0.1 \mathrm{mmHg})$ for $12 \mathrm{~h}$ and stored in a drying oven at $150{ }^{\circ} \mathrm{C}(760 \mathrm{mmHg})$; the molecular sieves were flame dried under vacuum $(0.1 \mathrm{mmHg})$ for $4-5 \mathrm{~min}$ immediately prior to use. The molarity of $n$-butyllithium solutions was determined by titration against diphenylacetic acid as an indicator (average of three determinations). ${ }^{2}$ The concentration of freshly prepared dimethyldioxirane solutions ${ }^{3}$ was determined by iodometric titration as follows: $\mathrm{A} 0.02 \mathrm{M}$ aqueous stock solution of sodium thiosulfate pentahydrate $\left(124 \mathrm{mg} \mathrm{Na} \mathrm{S}_{2} \mathrm{O}_{3} .5 \mathrm{H}_{2} \mathrm{O}\right.$ in $25 \mathrm{~mL} \mathrm{H} \mathrm{H}_{2} \mathrm{O}$ ) was prepared in a $25 \mathrm{~mL}$ graduated cylinder. A $100 \mathrm{~mL}$ flask was charged with water $(30 \mathrm{~mL})$, sodium iodide $(2.00 \mathrm{~g})$ and glacial acetic acid $(1 \mathrm{~mL})$, whereupon the dimethyldioxirane solution $(2 \mathrm{~mL})$ was added. The resulting brown mixture was rapidly titrated with the sodium thiosulfate stock solution until disappearance of the yellow iodine colour occurred. The concentration of the dimethyldioxirane solution was calculated according to the following equation:

$$
c(D M D O)=\frac{M(\text { titrant }) \times V(\text { titrant })}{V(D M D O) \times 2}
$$

and was generally in the range of $0.04 \mathrm{M}$ to $0.07 \mathrm{M}$.

\section{$1.2 \quad$ NMR spectroscopy}

NMR spectra were measured on a Bruker Avance III HD $800 \mathrm{MHz}$ spectrometer equipped with a CryoProbe $^{\mathrm{TM}}$, Bruker Avance III HD $400 \mathrm{MHz}$ spectrometer equipped with a CryoProbe ${ }^{\mathrm{TM}}$, Bruker AXR300, Varian VXR400 S and Bruker AMX600 spectrometers operating at $800 \mathrm{MHz}, 400 \mathrm{MHz}, 300 \mathrm{MHz}, 400 \mathrm{MHz}$ and $600 \mathrm{MHz}$ for proton nuclei $(200 \mathrm{MHz}, 100 \mathrm{MHz}, 75 \mathrm{MHz}, 100 \mathrm{MHz}, 150 \mathrm{MHz}$ for carbon nuclei), 
respectively. Proton chemical shifts are expressed in parts per million (ppm, $\delta$ scale) and are referenced to residual protium in the NMR solvent $\left(\mathrm{CHCl}_{3}: \delta 7.26, \mathrm{CDHCl}_{2}: \delta 5.32, \mathrm{C}_{6} \mathrm{HD}_{5}: 7.16\right)$. Carbon chemical shifts are expressed in parts per million ( $\delta$ scale, assigned carbon atom) and are referenced to the carbon resonance of the NMR solvent $\left(\mathrm{CDCl}_{3}: \delta 77.16, \mathrm{CD}_{2} \mathrm{Cl}_{2}: \delta 54.00, \mathrm{C}_{6} \mathrm{D}_{6}: 128.06\right) .{ }^{1} \mathrm{H}$ NMR spectroscopic data are reported as follows: Chemical shift in ppm (multiplicity, coupling constants $J(\mathrm{~Hz})$, integration intensity, assigned proton) (e.g. " $5.21\left(\mathrm{t},{ }^{3} \mathrm{~J}_{9 / 8}=7.3 \mathrm{~Hz}, 1 \mathrm{H}, 9-\mathrm{H}\right)$ "). The multiplicities are abbreviated with $\mathrm{s}$ (singlet), br s (broad singlet), d (doublet), $\mathrm{t}$ (triplet), q (quartet), $\mathrm{p}$ (pentet), se (sextet), $\mathrm{h}$ (heptet) and $\mathrm{m}$ (multiplet). In case of combined multiplicities, the multiplicity with the larger coupling constant is stated first. Except for multiplets, the chemical shift of all signals, as well for centrosymmetric multiplets, is reported as the center of the resonance range. Protons of diastereotopic methylene groups are reported as $\mathrm{X}-\mathrm{H}_{A}$ and $\mathrm{X}-\mathrm{H}_{B}$, where $\mathrm{X}-\mathrm{H}_{A}$ is the more downfield shifted proton. ${ }^{13} \mathrm{C}$ NMR spectroscopic data are reported as follows: Chemical shift in ppm (assigned carbon) (e.g. "159.22 (C-21)"). In cases were resonances overlap or cannot be unambiguously assigned to a single proton or carbon atom, multiple assignments are listed (e.g. the ${ }^{13} \mathrm{C}$ NMR assignment "18.29 (C-16, C-17), 17.84 (C-16, C-17)" indicates that the resonance at 18.29 is either $\mathrm{C}-16$ or $\mathrm{C}-17)$. Additionally to ${ }^{1} \mathrm{H}$ and ${ }^{13} \mathrm{C}$ NMR measurements, 2D NMR techniques such as homonuclear correlation spectroscopy (COSY), heteronuclear single quantum coherence (HSQC) and heteronuclear multiple bond coherence (HMBC) were used to assist signal assignment. For further elucidation of 3D structures of the products, nuclear Overhauser enhancement spectroscopy (NOESY) was conducted. All raw FID files were processed and the spectra analyzed using the program MestReNOVA 9.0 from Mestrelab Research S. L.

\subsection{Mass spectrometry}

All mass spectra were measured by the analytic section of the Department of Chemistry, LudwigMaximilians-Universität München. Mass spectra were recorded on the following spectrometers (ionisation mode in brackets): MAT 95 (EI) and MAT 90 (ESI) from Thermo Finnigan GmbH. Mass spectra were recorded in high-resolution. The method used is reported at the relevant section of the experimental section.

\section{$1.4 \quad$ IR spectroscopy}

IR spectra were recorded on a PerkinElmer Spectrum BX II FT-IR system. If required, substances were dissolved in dichloromethane prior to direct application on the ATR unit. Data are represented as follows: frequency of absorption $\left(\mathrm{cm}^{-1}\right)$, and intensity of absorption ( $\mathrm{s}=$ strong, $\mathrm{m}=$ medium, $\mathrm{w}=$ weak, $\mathrm{br}=$ broad).

\subsection{Optical rotation}

Optical rotation values were recorded on a PerkinElmer 241 or Anton Paar MCP 200 polarimeter. The specific rotation is calculated as follows:

$$
[\alpha]_{\lambda}^{\varphi}=\frac{[\alpha] \cdot 100}{c \cdot d}
$$

Thereby, the wavelength $\lambda$ is reported in $\mathrm{nm}$ and the measuring temperature $\varphi$ in ${ }^{\circ} \mathrm{C}$. $\alpha$ represents the recorded optical rotation, $c$ the concentration of the analyte in $10 \mathrm{mg} / \mathrm{mL}$ and $d$ the length of the cuvette in $\mathrm{dm}$. Thus, the specific rotation is given in $10^{-1} \cdot \mathrm{deg} \cdot \mathrm{cm}^{2} \cdot \mathrm{g}^{-1}$. Usage of the sodium $D$ line $(\lambda=589 \mathrm{~nm})$ is indicated by $D$ instead of the wavelength in $\mathrm{nm}$. The respective concentration as well as the solvent is reported at the relevant section of the experimental section. 


\section{Experimental Procedures}

\subsection{Synthesis of enone 31}

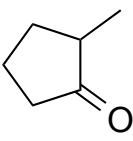

9 i. (S)-(-)- $\alpha$-methylbenzylamine, p-TsOH, toluene, $111^{\circ} \mathrm{C}, 5 \mathrm{~h}$

ii. MVK, $40^{\circ} \mathrm{C}, 4$ days

iii. $\mathrm{AcOH}, \mathrm{H}_{2} \mathrm{O}, 23^{\circ} \mathrm{C}, 2 \mathrm{~h}$

iv. $\mathrm{KOH}, \mathrm{EtOH}, 78^{\circ} \mathrm{C}, 2 \mathrm{~h}$

$(49 \%)$

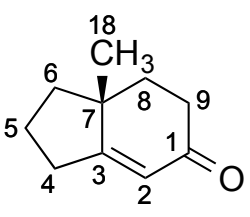

31

Based on the known preparation of enone $31,{ }^{4}$ a round-bottomed flask was charged with 2-methylcyclopentanone (9) $(28.0 \mathrm{~mL}, 260 \mathrm{mmol}, 1$ equiv), (S)-(-)- $\alpha$-methylbenzylamine (33.5 $\mathrm{mL}$, $260 \mathrm{mmol}, 1.00$ equiv), $p$-toluenesulfonic acid monohydrate (100 mg, $0.52 \mathrm{mmol}, 0.002$ equiv) and toluene $(50 \mathrm{~mL})$. The flask was then equipped with a Dean-Stark apparatus and the mixture was heated to $111^{\circ} \mathrm{C}$. After $5 \mathrm{~h}$, the removal of water was judged to be complete (approximately $4.3 \mathrm{~mL}$ water collected) and the mixture was cooled to $0{ }^{\circ} \mathrm{C}$. Methyl vinyl ketone $(25.3 \mathrm{~mL}, 312 \mathrm{mmol}, 1.20$ equiv) was added, and after $1 \mathrm{~h}$, the mixture was warmed to $40{ }^{\circ} \mathrm{C}$. After 2 days, a further portion of methyl vinyl ketone $\left(21.1 \mathrm{~mL}, 260 \mathrm{mmol}, 1.00\right.$ equiv) was added and stirring was continued at $40{ }^{\circ} \mathrm{C}$. After further 2 days, the mixture was cooled to $0{ }^{\circ} \mathrm{C}$, and a solution of acetic acid ( $22.3 \mathrm{~mL}, 389 \mathrm{mmol}, 1.50$ equiv) in water $(30 \mathrm{~mL})$ was added. The resulting biphasic mixture was warmed to $23{ }^{\circ} \mathrm{C}$ and stirred vigorously. After $2 \mathrm{~h}$, the mixture was diluted with saturated aqueous sodium chloride solution $(50 \mathrm{~mL})$, water $(50 \mathrm{~mL})$ and diethyl ether $(100 \mathrm{~mL})$. The layers were separated, the aqueous layer was extracted with diethyl ether $(5 \times 70 \mathrm{~mL})$ and the combined organic extracts were washed sequentially with aqueous $1 \mathrm{M}$ hydrogen chloride solution $(50 \mathrm{~mL})$ and saturated aqueous sodium chloride solution $(60 \mathrm{~mL})$. The washed solution was dried over sodium sulfate. The dried solution was filtered and the filtrate was concentrated to provide the diketone which was used in the next step without further purification.

A solution of the crude diketone (assuming $260 \mathrm{mmol}$ ) in ethanol $(280 \mathrm{~mL})$ was treated with potassium hydroxide ( $24.8 \mathrm{~g}, 442 \mathrm{mmol}, 1.70$ equiv) at $23^{\circ} \mathrm{C}$, and the resulting mixture was heated to $78{ }^{\circ} \mathrm{C}$. After $2 \mathrm{~h}$, the mixture was cooled to $0{ }^{\circ} \mathrm{C}$ and was acidified by addition of acetic acid (ca. $25 \mathrm{~mL}, \mathrm{pH} \sim 6$ ). The resulting mixture was concentrated until a thick paste resulted. Water $(150 \mathrm{~mL})$ and a mixture of diethyl ether-pentane $(1: 1 \mathrm{v} / \mathrm{v}, 100 \mathrm{~mL})$ was added. The layers were separated, the aqueous layer was extracted with diethyl ether-pentane $(1: 1 \mathrm{v} / \mathrm{v}, 4 \times 70 \mathrm{~mL})$ and the combined organic extracts were washed sequentially with water $(50 \mathrm{~mL})$ and saturated aqueous sodium chloride solution $(50 \mathrm{~mL})$. The washed solution was dried over sodium sulfate. The dried solution was filtered and the filtrate was concentrated. The residue was purified by flash-column chromatography on silica gel $(20 \% \rightarrow 30 \%$ ethyl acetate in hexanes) to provide enone $\mathbf{3 1}(19.0 \mathrm{~g}, 49 \%)$ as an orange oil. The characterization data for $\mathbf{3 1}$ were in agreement with values previously reported. ${ }^{4 a, 5}$ The absolute configuration $(R)$ of enone $\mathbf{3 1}$ was derived from the sign of the optical rotation, and $\mathbf{3 1}$ was estimated to be of $\geq 80 \%$ ee by comparison with the literature values (Lit.: ${ }^{4 a}[\alpha]_{D}^{20}=-108^{\circ}\left(\mathrm{c}=3.50\right.$, EtOH); Lit.: ${ }^{5}[\alpha]_{D}^{20}=-94^{\circ}(\mathrm{c}=1.00, \mathrm{EtOH})$ ).

TLC (20\% ethyl acetate in hexanes): $\mathrm{R}_{f}=0.32$ (UV/CAM). ${ }^{1} \mathrm{H}$ NMR $\left(400 \mathrm{MHz}, \mathrm{CDCl}_{3}\right) \delta 5.78-5.76(\mathrm{~m}, 1 \mathrm{H}$, 2-H), 2.76-2.64 (m, $\left.1 \mathrm{H}, 4-\mathrm{H}_{\mathrm{A}}\right), 2.61-2.42\left(\mathrm{~m}, 2 \mathrm{H}, 4-\mathrm{H}_{\mathrm{B}}, 9-\mathrm{H}_{\mathrm{A}}\right), 2.39-2.31\left(\mathrm{~m}, 1 \mathrm{H}, 9-\mathrm{H}_{\mathrm{B}}\right), 2.03\left(\mathrm{ddd},{ }^{2} J_{8 \mathrm{~A} / 8 \mathrm{~B}}=\right.$ $\left.13.0 \mathrm{~Hz},{ }^{3} \mathrm{~J}_{8 \mathrm{~A} / 9 \mathrm{~B}}=5.3 \mathrm{~Hz},{ }^{3} \mathrm{~J}_{8 \mathrm{~A} / 9 \mathrm{~A}}=2.1 \mathrm{~Hz}, 1 \mathrm{H}, 8-\mathrm{H}_{\mathrm{A}}\right), 1.97-1.75\left(\mathrm{~m}, 4 \mathrm{H}, 5-\mathrm{H}, 6-\mathrm{H}_{\mathrm{A}}, 8-\mathrm{H}_{\mathrm{B}}\right), 1.53-1.43(\mathrm{~m}, 1 \mathrm{H}$, 6- $\left.\mathrm{H}_{\mathrm{B}}\right), 1.16(\mathrm{~s}, 3 \mathrm{H}, 18-\mathrm{H}) .{ }^{13} \mathrm{C} \mathrm{NMR}\left(100 \mathrm{MHz}, \mathrm{CDCl}_{3}\right) \delta 199.79$ (C-1), 178.76 (C-3), 121.42 (C-2), 42.82 (C-7), 40.94 (C-6), 36.17 (C-8), 33.93 (C-9), 30.84 (C-4), 22.49 (C-18), 21.26 (C-5). IR (Diamond-ATR, neat) $\tilde{v}_{\max }$ : $2960(\mathrm{~m}), 2928(\mathrm{~m}), 2861(\mathrm{~m}), 1667$ (s), $1454(\mathrm{w}), 1421(\mathrm{w}), 1296(\mathrm{w}), 1222(\mathrm{w}), 1202(\mathrm{w}) \mathrm{cm}^{-1}$. HRMS (EI): calcd for ([M], $\left.\mathrm{C}_{10} \mathrm{H}_{14} \mathrm{O}\right)^{+}: 150.1045$, found: 150.1044 . $[\alpha]_{D}^{20}=-94.8^{\circ}(\mathrm{c}=2.00$, EtOH). 


\subsection{Synthesis of ketal 32}

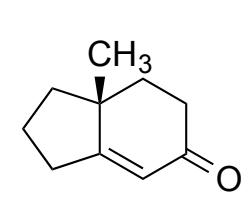

31

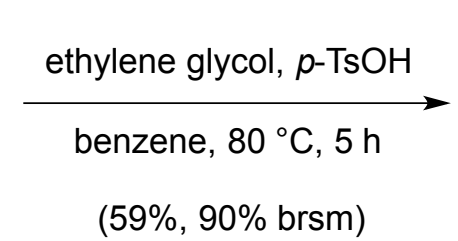

(59\%, 90\% brsm)

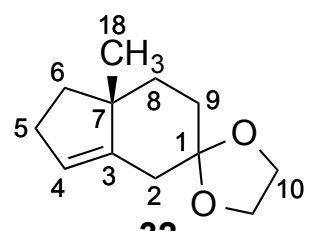

32

Based on the known preparation of ketal $32,{ }^{6}$ a round-bottomed flask was charged with enone 31 (9.21 g, $61.3 \mathrm{mmol}, 1$ equiv), ethylene glycol ( $20.5 \mathrm{~mL}, 368 \mathrm{mmol}, 6.00$ equiv), $p$-toluenesulfonic acid monohydrate $(1.17 \mathrm{~g}, 6.13 \mathrm{mmol}, 0.10$ equiv) and benzene $(150 \mathrm{~mL})$. The flask was equipped with a Dean-Stark apparatus and the mixture was heated to $80^{\circ} \mathrm{C}$. After $5 \mathrm{~h}$, the removal of water was judged to be complete and the mixture was cooled to $23^{\circ} \mathrm{C}$. The mixture was diluted with saturated aqueous sodium hydrogen carbonate solution $(150 \mathrm{~mL})$. The layers were separated, the aqueous layer was extracted with diethyl ether $(3 \times 70 \mathrm{~mL})$ and the combined organic extracts were washed with saturated aqueous sodium chloride solution $(100 \mathrm{~mL})$. The washed solution was dried over sodium sulfate. The dried solution was filtered and the filtrate was concentrated. The residue was purified by flash-column chromatography on silica gel (15\% $\rightarrow 30 \%$ ethyl acetate in hexanes) to provide recovered enone $\mathbf{3 1}$ $(3.19 \mathrm{~g}, 35 \%)$ and ketal $32(7.00 \mathrm{~g}, 59 \%, 90 \% \mathrm{brsm})$ as a yellow oil. The ${ }^{1} \mathrm{H} N M R,{ }^{13} \mathrm{C} N M R$, IR and HMRS data for ketal $\mathbf{3 2}$ were in full agreement with values previously reported for the racemic compound. ${ }^{7}$

$\operatorname{TLC}\left(20 \%\right.$ ethyl acetate in hexanes): $\mathrm{R}_{f}=0.53$ (CAM). ${ }^{1} \mathrm{H}$ NMR $\left(400 \mathrm{MHz}, \mathrm{CDCl}_{3}\right) \delta 5.32-5.28(\mathrm{~m}, 1 \mathrm{H}, 4-\mathrm{H})$, 4.05-3.88 (m, 4H, 10-H), 2.48-2.19 (m, 4H, 2-H, 5-H), 1.91-1.74 (m, 2H, 6- $\left.\mathrm{H}_{\mathrm{A}}, 9-\mathrm{H}_{\mathrm{A}}\right), 1.75-1.61(\mathrm{~m}, 3 \mathrm{H}$, 6- $\left.\mathrm{H}_{\mathrm{B}}, 8-\mathrm{H}_{\mathrm{A}}, 9-\mathrm{H}_{\mathrm{B}}\right), 1.59-1.49\left(\mathrm{~m}, 1 \mathrm{H}, 8-\mathrm{H}_{\mathrm{B}}\right), 1.06(\mathrm{~s}, 3 \mathrm{H}, 18-\mathrm{H}) .{ }^{13} \mathrm{C} \mathrm{NMR}\left(100 \mathrm{MHz}, \mathrm{CDCl}_{3}\right) \delta 146.40(\mathrm{C}-3)$, 122.63 (C-4), 109.87 (C-1), 64.65 (C-10), 64.51 (C-10'), 45.14 (C-7), 40.31 (C-6), 37.72 (C-8), 36.25 (C-2), 31.86 (C-9), 30.51 (C-5), 22.36 (C-18). IR (Diamond-ATR, neat) $\tilde{v}_{\max }: 2944$ (s), 2880 (m), 1455 (w), $1353(w), 1306(w), 1257(w), 1177(w), 1115(\mathrm{~m}), 1090(\mathrm{~s}), 1020(\mathrm{~m}) \mathrm{cm}^{-1}$. HRMS (EI): calcd for ([M], $\left.\mathrm{C}_{12} \mathrm{H}_{18} \mathrm{O}_{2}\right)^{+}:$194.1307, found: 194.1293. $[\alpha]_{D}^{20}=-18.7^{\circ}\left(\mathrm{c}=0.83, \mathrm{CH}_{2} \mathrm{Cl}_{2}\right.$ ). 


\subsection{Synthesis of diol 33}

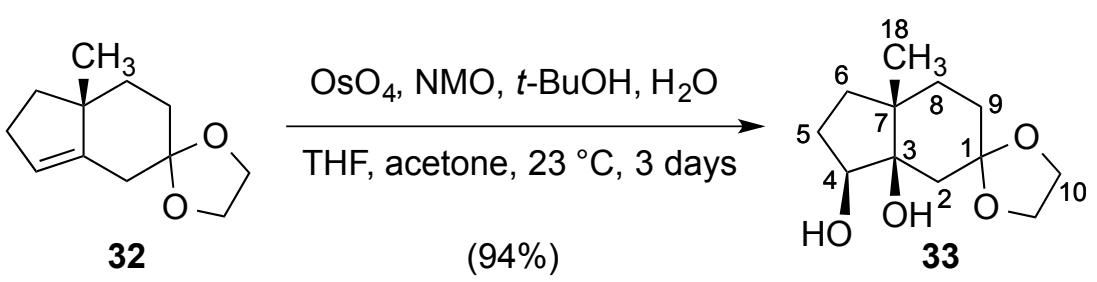

Based on a slightly modified literature procedure, ${ }^{7}$ a solution of ketal 32 ( $8.10 \mathrm{~g}, 41.7 \mathrm{mmol}, 1$ equiv) in tetrahydrofuran $(24 \mathrm{~mL})$, tert-butanol $(70 \mathrm{~mL})$, water $(12 \mathrm{~mL})$ and acetone $(12 \mathrm{~mL})$ was treated sequentially with 4-methylmorpholine $N$-oxide $(5.86 \mathrm{~g}, 50.0 \mathrm{mmol}, 1.20$ equiv) and osmium tetroxide solution ( 2.5 wt.\% in $t$ - $\mathrm{BuOH}, 10.2 \mathrm{~mL}, 1.04 \mathrm{mmol}, 0.03$ equiv) at $23{ }^{\circ} \mathrm{C}$. After 3 days, sodium sulfite $(15.8 \mathrm{~g}, 125 \mathrm{mmol}, 3.00$ equiv) and water $(20 \mathrm{~mL})$ were added. After $15 \mathrm{~min}$, the mixture was diluted with more water $(100 \mathrm{~mL})$ and ethyl acetate $(100 \mathrm{~mL})$. The layers were separated, the aqueous layer was extracted with ethyl acetate $(6 \times 100 \mathrm{~mL})$ and the combined organic extracts were washed with saturated aqueous sodium chloride solution $(100 \mathrm{~mL})$. The washed solution was dried over sodium sulfate. The dried solution was filtered and the filtrate was concentrated. The residue was purified by flash-column chromatography on silica gel (7\% methanol in dichloromethane) to provide diol $\mathbf{3 3}$ (8.94 g, $94 \%$ ) as a brownish oil which solidified upon standing. The ${ }^{1} \mathrm{H} N M R,{ }^{13} \mathrm{C} N M R$, IR and HMRS data for diol 33 were in full agreement with values previously reported for the racemic compound. ${ }^{7}$

TLC (5\% methanol in dichloromethane): $\mathrm{R}_{f}=0.24$ (CAM). ${ }^{1} \mathrm{H}$ NMR $\left(400 \mathrm{MHz}, \mathrm{CDCl}_{3}\right) \delta 4.22-4.15(\mathrm{~m}, 1 \mathrm{H}$, 4-H), 4.00-3.89 (m, 4H, 10-H), $2.96(\mathrm{br} \mathrm{s}, 1 \mathrm{H}, 3-\mathrm{OH}), 2.49\left(\mathrm{~d},{ }^{3} J_{4 \mathrm{OH} / 4}=3.9 \mathrm{~Hz}, 1 \mathrm{H}, 4-\mathrm{OH}\right), 2.13-2.02(\mathrm{~m}, 1 \mathrm{H}$, $\left.5-\mathrm{H}_{\mathrm{A}}\right), 1.76-1.42\left(\mathrm{~m}, 9 \mathrm{H}, 2-\mathrm{H}, 5-\mathrm{H}_{\mathrm{B}}, 6-\mathrm{H}, 8-\mathrm{H}, 9-\mathrm{H}\right), 1.07(\mathrm{~s}, 3 \mathrm{H}, 18-\mathrm{H}) .{ }^{13} \mathrm{C} \mathrm{NMR}\left(100 \mathrm{MHz}, \mathrm{CDCl}_{3}\right)$ $\delta 109.24$ (C-1), 80.23 (C-3), 76.67 (C-4), 64.50 (C-10), 64.27 (C-10'), 42.45 (C-7), 40.25 (C-2), 34.02 (C-6), 32.54 (C-8), 30.45 (C-9), 28.82 (C-5), 21.58 (C-18). IR (Diamond-ATR, neat) $\tilde{v}_{\max }: 3460$ (br), 2954 (m), $1465(\mathrm{~m}), 1428(\mathrm{~m}), 1341(\mathrm{~m}), 1265(\mathrm{~s}), 1122(\mathrm{~m}), 1091(\mathrm{~s}), 1069(\mathrm{~s}), 1032(\mathrm{~s}), 1012(\mathrm{~m}) \mathrm{cm}^{-1}$. HRMS (ESI): calcd for $\left([\mathrm{M}+\mathrm{Na}], \mathrm{C}_{12} \mathrm{H}_{20} \mathrm{O}_{4} \mathrm{Na}\right)^{+}: 251.12593$, found: $251.12550 .[\alpha]_{D}^{20}=-61.8^{\circ}\left(\mathrm{c}=0.33, \mathrm{CH}_{2} \mathrm{Cl}_{2}\right.$ ). 


\subsection{Synthesis of hydrindanone 34}

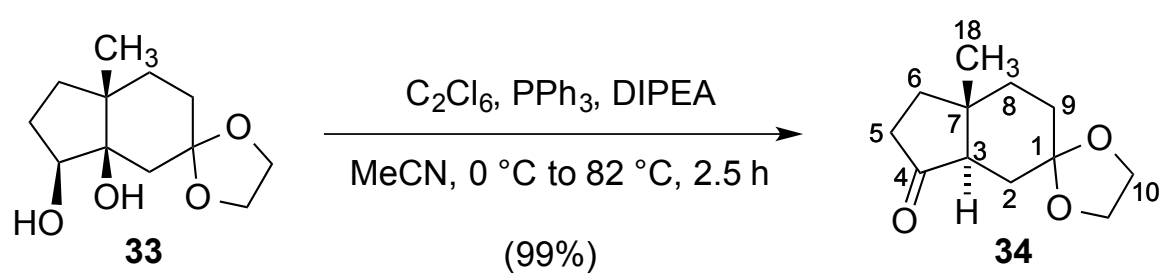

Based on the literature procedure, ${ }^{7}$ a suspension of triphenylphosphine (17.3 g, $66.1 \mathrm{mmol}, 2.20$ equiv) in acetonitrile $(200 \mathrm{~mL})$ was treated with hexachloroethane $\left(15.7 \mathrm{~g}, 66.1 \mathrm{mmol}, 2.20\right.$ equiv) at $0{ }^{\circ} \mathrm{C}$. After $5 \mathrm{~min}$, the cooling bath was removed and the mixture was warmed to $23^{\circ} \mathrm{C}$. After $30 \mathrm{~min}$, the mixture was cooled to $0{ }^{\circ} \mathrm{C}$, whereupon $\mathrm{N}, \mathrm{N}$-diisopropylethylamine $(22.9 \mathrm{~mL}, 132 \mathrm{mmol}, 4.40$ equiv), followed by a solution of diol 33 (6.86 g, $30 \mathrm{mmol}, 1$ equiv) in acetonitrile $(120 \mathrm{~mL})$ was added dropwise over a period of $10 \mathrm{~min}$. After $1 \mathrm{~h}$, the reaction flask was placed in a preheated $\left(82^{\circ} \mathrm{C}\right)$ oil bath. After $1.5 \mathrm{~h}$, the mixture was allowed to cool to $23{ }^{\circ} \mathrm{C}$, and then was diluted with diethyl ether $(300 \mathrm{~mL})$. The organic layer was washed with water $(2 \times 100 \mathrm{~mL})$. The washed solution was dried over sodium sulfate. The dried solution was filtered and the filtrate was concentrated. The residue was purified by flash-column chromatography on silica gel (30\% ethyl acetate in hexanes) to provide hydrindanone $\mathbf{3 4}(6.32 \mathrm{~g}, \geq 99 \%)$ as a yellowish oil which solidified upon standing. The ${ }^{1} \mathrm{H} N M R,{ }^{13} \mathrm{C} N M R, I R$ and HMRS data for hydrindanone $\mathbf{3 4}$ were in full agreement with values previously reported for the racemic compound. ${ }^{7}$

TLC (30\% ethyl acetate in hexanes): $\mathrm{R}_{f}=0.38$ (CAM). ${ }^{1} \mathrm{H}$ NMR $\left(400 \mathrm{MHz}, \mathrm{CDCl}_{3}\right) \delta 4.02-3.85(\mathrm{~m}, 4 \mathrm{H}, 10-\mathrm{H})$, 2.40-2.20 (m, 3H, 3-H, 5-H), 1.98-1.91 (m, 1H, 2- $\left.\mathrm{H}_{\mathrm{A}}\right), 1.90-1.57(\mathrm{~m}, 6 \mathrm{H}, 6-\mathrm{H}, 8-\mathrm{H}, 9-\mathrm{H}), 1.45$ (app t, $\left.{ }^{2} J_{2 \mathrm{~B} / 2 \mathrm{~A}}={ }^{3} J_{2 \mathrm{~B} / 3}=13.1 \mathrm{~Hz}, 1 \mathrm{H}, 2-\mathrm{H}_{\mathrm{B}}\right), 0.87(\mathrm{~s}, 3 \mathrm{H}, 18-\mathrm{H}) .{ }^{13} \mathrm{C} \mathrm{NMR}\left(100 \mathrm{MHz}, \mathrm{CDCl}_{3}\right) \delta 216.44(\mathrm{C}-4), 109.56$ (C1), 64.62 (C-10), 64.33 (C-10'), 57.34 (C-3), 38.72 (C-7), 35.93 (C-5, C-6, C-8), 35.88 (C-5, C-6, C-8), 35.39 (C-5, C-6, C-8), 31.70 (C-9), 29.86 (C-2), 17.03 (C-18). IR (Diamond-ATR, neat) $\tilde{v}_{\text {max }}: 2959$ (m), 2882 $(\mathrm{m}), 1737(\mathrm{~s}), 1459(\mathrm{~m}), 1354(\mathrm{~m}), 1287(\mathrm{~m}), 1194(\mathrm{~m}), 1110(\mathrm{~s}), 1080(\mathrm{~m}), 1016(\mathrm{~m}) \mathrm{cm}^{-1}$. HRMS (ESI): calcd for $\left([\mathrm{M}+\mathrm{H}], \mathrm{C}_{12} \mathrm{H}_{19} \mathrm{O}_{3}\right)^{+}: 211.13342$, found: $211.13297 .[\alpha]_{D}^{20}=-92.6^{\circ}\left(\mathrm{c}=1.00, \mathrm{CH}_{2} \mathrm{Cl}_{2}\right.$ ). 


\subsection{Synthesis of trans-hydrindane 10}

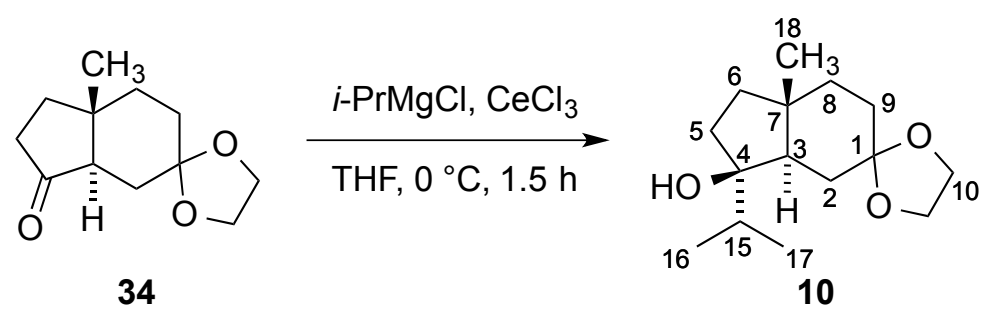

Based on a slightly modified literature procedure, ${ }^{7}$ a solution of hydrindanone $34(6.31 \mathrm{~g}, 30 \mathrm{mmol}$, 1 equiv) in tetrahydrofuran $(250 \mathrm{~mL})$ was treated with anhydrous cerium(III) trichloride $(11.1 \mathrm{~g}, 45 \mathrm{mmol}$, 1.50 equiv) at $23{ }^{\circ} \mathrm{C}$. After $1.5 \mathrm{~h}$, the colourless suspension was cooled to $0{ }^{\circ} \mathrm{C}$, whereupon a solution of isopropylmagnesium chloride ( $2 \mathrm{M}$ in THF, $33.8 \mathrm{~mL}, 67.5 \mathrm{mmol}, 2.25$ equiv) was added over a period of $10 \mathrm{~min}$. After $1.5 \mathrm{~h}$, the mixture was diluted with $\mathrm{pH} 7$ buffer solution $(300 \mathrm{~mL})$ and diethyl ether $(100 \mathrm{~mL})$. The layers were separated, the aqueous layer was extracted with diethyl ether $(3 \times 70 \mathrm{~mL})$ and the combined organic extracts were washed with saturated aqueous sodium chloride solution (100 $\mathrm{mL}$ ). The washed solution was dried over sodium sulfate. The dried solution was filtered through a pad of Celite and the filtrate was concentrated to provide trans-hydrindane $\mathbf{1 0}(8.10 \mathrm{~g})$ as a brownish oil which was used in the next step without further purification.

An analytically pure sample of trans-hydrindane $\mathbf{1 0}$ was obtained by flash-column chromatography on silica gel (30\% ethyl acetate in hexanes). The ${ }^{1} \mathrm{H}$ NMR, ${ }^{13} \mathrm{C} N M R, I R$ and HMRS data for trans-hydrindane 10 were in full agreement with values previously reported for the racemic compound. ${ }^{7}$

TLC (30\% ethyl acetate in hexanes): $\mathrm{R}_{f}=0.37$ (CAM). ${ }^{1} \mathrm{H}$ NMR $\left(400 \mathrm{MHz}, \mathrm{CDCl}_{3}\right) \delta 3.98-3.91(\mathrm{~m}, 4 \mathrm{H}, 10-\mathrm{H})$, $2.00\left(\mathrm{dd},{ }^{2} J_{5 \mathrm{~A} / 5 \mathrm{~B}}=14.3 \mathrm{~Hz},{ }^{3} J_{5 \mathrm{~A} / 6 \mathrm{~B}}=9.6 \mathrm{~Hz}, 1 \mathrm{H}, 5-\mathrm{H}_{\mathrm{A}}\right), 1.86-1.52\left(\mathrm{~m}, 9 \mathrm{H}, 2-\mathrm{H}, 3-\mathrm{H}, 5-\mathrm{H}_{\mathrm{B}}, 6-\mathrm{H}_{\mathrm{A}}, 8-\mathrm{H}_{\mathrm{A}}, 9-\mathrm{H}\right.$, $15-\mathrm{H}), 1.38\left(\mathrm{app} \mathrm{td},{ }^{2} J_{8 \mathrm{~B} / 8 \mathrm{~A}}={ }^{3} J_{8 \mathrm{~B} / 9 \mathrm{~A}}=13.4 \mathrm{~Hz},{ }^{3} \mathrm{~J}_{8 \mathrm{~B} / 9 \mathrm{~B}}=4.3 \mathrm{~Hz}, 1 \mathrm{H}, 8-\mathrm{H}_{\mathrm{B}}\right), 1.16-1.05\left(\mathrm{~m}, 4 \mathrm{H}, 6-\mathrm{H}_{\mathrm{B}}, 18-\mathrm{H}\right)$, $1.04(\mathrm{~s}, 1 \mathrm{H}, 4-\mathrm{OH}), 0.95\left(\mathrm{~d},{ }^{3} \mathrm{~J}=6.8 \mathrm{~Hz}, 3 \mathrm{H}, 16-\mathrm{H}, 17-\mathrm{H}\right), 0.90\left(\mathrm{~d},{ }^{3} \mathrm{~J}=6.8 \mathrm{~Hz}, 3 \mathrm{H}, 16-\mathrm{H}, 17-\mathrm{H}\right) .{ }^{13} \mathrm{C}$ NMR $\left(100 \mathrm{MHz}, \mathrm{CDCl}_{3}\right) \delta 110.81$ (C-1), 83.30 (C-4), 64.47 (C-10), 64.29 (C-10'), 51.09 (C-3), 41.56 (C-7), 39.42 (C-6), 37.47 (C-15), 37.29 (C-8), 36.73 (C-5), 31.61 (C-2, C-9), 31.56 (C-2, C-9), 18.47 (C-16, C-17), 18.31 (C-18), 17.65 (C-16, C-17). IR (Diamond-ATR, neat) $\tilde{v}_{\text {max }}: 3508$ (br), 2953 (s), 2877 (s), 1459 (m), $1348(\mathrm{~m}), 1292(\mathrm{~m}), 1254(\mathrm{~m}), 1192(\mathrm{~m}), 1145(\mathrm{~s}), 1089(\mathrm{~s}), 1020(\mathrm{~m}) \mathrm{cm}^{-1}$. HRMS (EI): calcd for ([M], $\left.\mathrm{C}_{15} \mathrm{H}_{26} \mathrm{O}_{3}\right)^{+}: 254.1882$, found: 254.1876 . $[\alpha]_{D}^{20}=-12.6^{\circ}\left(\mathrm{c}=1.00, \mathrm{CH}_{2} \mathrm{Cl}_{2}\right.$ ). 


\subsection{Synthesis of benzyl ether 35}

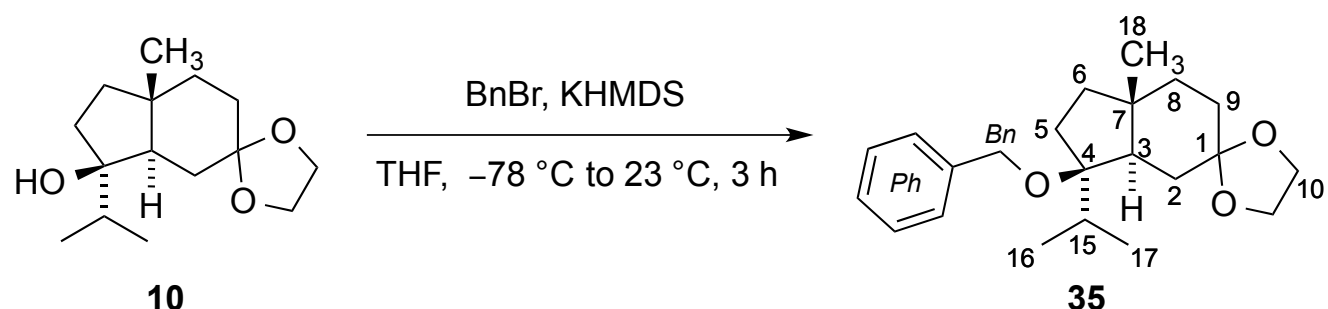

10

35

A solution of crude trans-hydrindane $10(6.50 \mathrm{~g}, 25.6 \mathrm{mmol}, 1$ equiv) in tetrahydrofuran ( $21 \mathrm{~mL}$ ) was treated sequentially with potassium bis(trimethylsilyl)amide solution $(1 \mathrm{M}$ in THF, $30.7 \mathrm{~mL}, 30.7 \mathrm{mmol}$, 1.20 equiv) and benzyl bromide $\left(4.59 \mathrm{~mL}, 38.3 \mathrm{mmol}, 1.50\right.$ equiv) at $-78^{\circ} \mathrm{C}$. After $5 \mathrm{~min}$, the cooling bath was removed and the mixture was allowed to warm to $23^{\circ} \mathrm{C}$. After $3 \mathrm{~h}$, the mixture was diluted with saturated aqueous ammonium chloride solution $(150 \mathrm{~mL})$ and diethyl ether $(50 \mathrm{~mL})$. The layers were separated, the aqueous layer was extracted with diethyl ether $(3 \times 50 \mathrm{~mL})$ and the combined organic extracts were dried over sodium sulfate. The dried solution was filtered through a pad of Celite and the filtrate was concentrated to provide benzyl ether $\mathbf{3 5}(8.82 \mathrm{~g})$ as a yellowish oil which was used in the next step without further purification.

An analytically pure sample of benzyl ether $\mathbf{3 5}$ was obtained by flash-column chromatography on silica gel $(5 \% \rightarrow 10 \%$ ethyl acetate in hexanes).

TLC (10\% ethyl acetate in hexanes): $\mathrm{R}_{f}=0.33$ (UV/CAM). ${ }^{1} \mathrm{H}$ NMR $\left(400 \mathrm{MHz}, \mathrm{CDCl}_{3}\right) \delta 7.36-7.19(\mathrm{~m}, 5 \mathrm{H}$, $P h), 4.45-4.37(\mathrm{~m}, 2 \mathrm{H}, B n), 3.98-3.92(\mathrm{~m}, 4 \mathrm{H}, 10-\mathrm{H}), 2.33-2.20(\mathrm{~m}, 1 \mathrm{H}, 15-\mathrm{H}), 2.22-2.11\left(\mathrm{~m}, 1 \mathrm{H}, 5-\mathrm{H}_{\mathrm{A}}\right)$, 1.95-1.75 (m, 5H, 2-H, 3-H, 5- $\left.\mathrm{H}_{B}, 9-\mathrm{H}_{\mathrm{A}}\right), 1.66-1.53\left(\mathrm{~m}, 3 \mathrm{H}, 6-\mathrm{H}_{\mathrm{A}}, 8-\mathrm{H}_{\mathrm{A}}, 9-\mathrm{H}_{\mathrm{B}}\right), 1.41\left(\mathrm{app} \mathrm{td},{ }^{2} \mathrm{~J}_{8 \mathrm{~B} / 8 \mathrm{~A}}={ }^{3} \mathrm{~J}_{8 \mathrm{~B} / 9 \mathrm{~A}}=\right.$ $\left.13.4 \mathrm{~Hz},{ }^{3} J_{8 \mathrm{~B} / 9 \mathrm{~B}}=4.5 \mathrm{~Hz}, 1 \mathrm{H}, 8-\mathrm{H}_{\mathrm{B}}\right), 1.20-1.09\left(\mathrm{~m}, 1 \mathrm{H}, 6-\mathrm{H}_{\mathrm{B}}\right), 1.07(\mathrm{~s}, 3 \mathrm{H}, 18-\mathrm{H}), 0.97\left(\mathrm{~d},{ }^{3} \mathrm{~J}=6.8 \mathrm{~Hz}, 3 \mathrm{H}\right.$, $16-\mathrm{H}, 17-\mathrm{H}), 0.95\left(\mathrm{~d},{ }^{3} \mathrm{~J}=6.8 \mathrm{~Hz}, 3 \mathrm{H}, 16-\mathrm{H}, 17-\mathrm{H}\right) .{ }^{13} \mathrm{C}$ NMR $\left(100 \mathrm{MHz}, \mathrm{CDCl}_{3}\right) \delta 140.34(P h), 128.23(P h)$, $126.79(P h), 126.68(P h), 111.05$ (C-1), 87.58 (C-4), 64.40 (C-10), 64.28 (C-10'), $62.43(B n), 48.33$ (C-3), 41.86 (C-7), 40.17 (C-6), 36.87 (C-8), 34.43 (C-5), 33.75 (C-2), 33.01 (C-15), 31.74 (C-9), 18.48 (C-16, C-17), 18.19 (C-18), 18.15 (C-16, C-17). IR (Diamond-ATR, neat) $\tilde{v}_{\text {max }}: 2955(\mathrm{~s}), 2877(\mathrm{~m}), 1454(\mathrm{~m}), 1385(\mathrm{~m})$, $1263(\mathrm{~m}), 1198(\mathrm{~m}), 1143(\mathrm{~m}), 1085$ (s), 1061 (s), $1040(\mathrm{~s}) \mathrm{cm}^{-1}$. HRMS (El): calcd for ([M], $\left.\mathrm{C}_{22} \mathrm{H}_{32} \mathrm{O}_{3}\right)^{+}$: 344.2351, found: $344.2338 .[\alpha]_{D}^{20}=+32.2^{\circ}\left(\mathrm{c}=1.06, \mathrm{CH}_{2} \mathrm{Cl}_{2}\right)$. 


\subsection{Synthesis of ketone 11}<smiles>CC(C)[C@@]12CCC3(C[C@H]1[C@](C)(O)CC2)OCCO3</smiles>

35

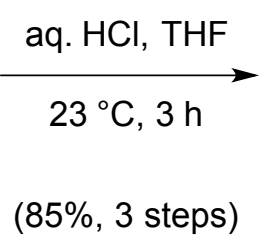

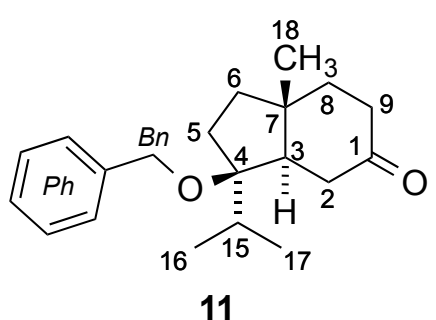

11

A solution of crude benzyl ether 35 ( $8.82 \mathrm{~g}, 25.6 \mathrm{mmol}, 1$ equiv) in tetrahydrofuran ( $100 \mathrm{~mL}$ ) was treated with aqueous $4 \mathrm{M}$ hydrogen chloride solution $(30 \mathrm{~mL}$ ) and the resulting mixture was stirred vigorously at $23{ }^{\circ} \mathrm{C}$. After $3 \mathrm{~h}$, the mixture was diluted with water $(50 \mathrm{~mL})$ and diethyl ether $(50 \mathrm{~mL})$. The layers were separated, the aqueous layer was extracted with diethyl ether $(3 \times 50 \mathrm{~mL})$ and the combined organic extracts were washed sequentially with saturated aqueous sodium hydrogen carbonate solution $(50 \mathrm{~mL})$ and saturated aqueous sodium chloride solution $(50 \mathrm{~mL})$. The washed solution was dried over sodium sulfate. The dried solution was filtered and the filtrate was concentrated. The residue was purified by flash-column chromatography on silica gel ( $10 \% \rightarrow 20 \%$ ethyl acetate in hexanes) to provide ketone $\mathbf{1 1}$ ( $6.51 \mathrm{~g}, 85 \%$ over three steps) as a yellowish oil which solidified upon standing.

TLC (10\% ethyl acetate in hexanes): $\mathrm{R}_{f}=0.29$ (UV/CAM). ${ }^{1} \mathrm{H}$ NMR $\left(600 \mathrm{MHz}, \mathrm{CDCl}_{3}\right) \delta 7.29-7.16(\mathrm{~m}, 5 \mathrm{H}$, $P h), 4.39-4.33(\mathrm{~m}, 2 \mathrm{H}, \mathrm{Bn}), 2.70\left(\mathrm{dd},{ }^{2} \mathrm{~J}_{2 \mathrm{~A} / 2 \mathrm{~B}}=16.1 \mathrm{~Hz},{ }^{3} \mathrm{~J}_{2 \mathrm{~A} / 3}=14.2 \mathrm{~Hz}, 1 \mathrm{H}, 2-\mathrm{H}_{\mathrm{A}}\right), 2.44-2.36\left(\mathrm{~m}, 2 \mathrm{H}, 2-\mathrm{H}_{\mathrm{B}}\right.$, $\left.9-\mathrm{H}_{\mathrm{A}}\right), 2.33-2.28\left(\mathrm{~m}, 1 \mathrm{H}, 9-\mathrm{H}_{\mathrm{B}}\right), 2.28-2.16\left(\mathrm{~m}, 2 \mathrm{H}, 5-\mathrm{H}_{\mathrm{A}}, 15-\mathrm{H}\right), 1.85\left(\mathrm{dd},{ }^{2} J_{5 \mathrm{~B} / 5 \mathrm{~A}}=13.8 \mathrm{~Hz},{ }^{3} \mathrm{~J}_{5 \mathrm{~B} / 6 \mathrm{~B}}=8.1 \mathrm{~Hz}\right.$, $\left.1 \mathrm{H}, 5-\mathrm{H}_{\mathrm{B}}\right), 1.82-1.75\left(\mathrm{~m}, 2 \mathrm{H}, 3-\mathrm{H}, 8-\mathrm{H}_{\mathrm{A}}\right), 1.65\left(\mathrm{dd},{ }^{2} \mathrm{~J}_{6 \mathrm{~A} / 6 \mathrm{~B}}=12.0 \mathrm{~Hz},{ }^{3} \mathrm{~J}_{6 \mathrm{~A} / 5 \mathrm{~A}}=7.9 \mathrm{~Hz}, 1 \mathrm{H}, 6-\mathrm{H}_{\mathrm{A}}\right), 1.55-1.48(\mathrm{~m}$, $\left.1 \mathrm{H}, 8-\mathrm{H}_{\mathrm{B}}\right), 1.20-1.13\left(\mathrm{~m}, 4 \mathrm{H}, 6-\mathrm{H}_{\mathrm{B}}, 18-\mathrm{H}\right), 0.89\left(\mathrm{~d},{ }^{3} \mathrm{~J}=6.8 \mathrm{~Hz}, 3 \mathrm{H}, 16-\mathrm{H}, 17-\mathrm{H}\right), 0.86\left(\mathrm{~d},{ }^{3} \mathrm{~J}=6.8 \mathrm{~Hz}, 3 \mathrm{H}\right.$, 16-H, 17-H). ${ }^{13} \mathrm{C}$ NMR (150 MHz, CDCl $)$ ठ $213.61(\mathrm{C}-1), 139.84(P h), 128.34(P h), 127.05(P h), 126.84(P h)$, 87.40 (C-4), $62.61(B n), 49.63$ (C-3), 41.74 (C-7), 41.55 (C-2), 39.94 (C-6), 37.72 (C-9), 37.29 (C-8), 35.08 (C-5), 33.06 (C-15), 18.26 (C-16, C-17), 18.15 (C-18), 17.78 (C-16, C-17). IR (Diamond-ATR, neat) $\tilde{V}_{\text {max }}: 2958(\mathrm{~m}), 2876(\mathrm{~m}), 1706(\mathrm{~s}), 1454(\mathrm{~m}), 1387(\mathrm{~m}), 1220(\mathrm{w}), 1111(\mathrm{~m}), 1085(\mathrm{~m}), 1056(\mathrm{~s}), 1028(\mathrm{~m})$ $\mathrm{cm}^{-1}$. HRMS (ESI): calcd for $\left(\left[\mathrm{M}+\mathrm{NH}_{4}\right], \mathrm{C}_{20} \mathrm{H}_{32} \mathrm{O}_{2} \mathrm{~N}\right)^{+}: 318.24330$, found: $318.24334 .[\boldsymbol{\alpha}]_{\boldsymbol{D}}^{\mathbf{2 0}}=+15.6^{\circ}$ ( $c=1.05, \mathrm{CH}_{2} \mathrm{Cl}_{2}$ ). 


\subsection{Synthesis of silyl ether 36}

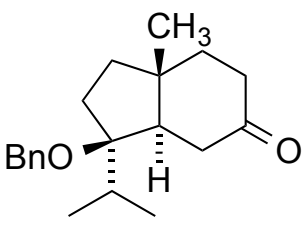

11

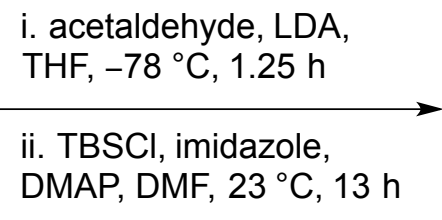

DMAP, DMF, $23^{\circ} \mathrm{C}, 13 \mathrm{~h}$

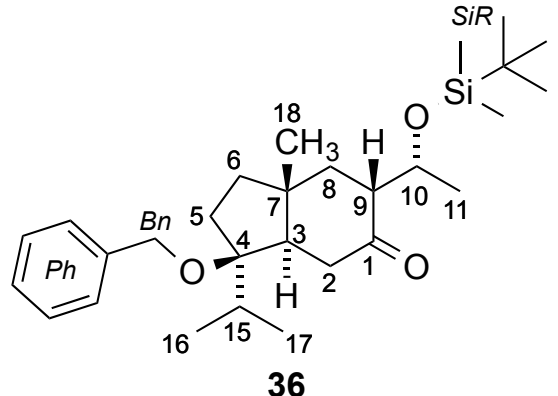

36

A solution of ketone 11 (1.28 g, $4.26 \mathrm{mmol}, 1$ equiv) in tetrahydrofuran (12 mL) was treated with a freshly prepared solution of lithium diisopropylamide (1 M in THF, $4.69 \mathrm{~mL}, 4.69 \mathrm{mmol}, 1.10$ equiv) at $-78{ }^{\circ} \mathrm{C}$ (addition to the inner wall of the reaction flask, such that the solution was cooled before reaching the reaction mixture). After $45 \mathrm{~min}$, a solution of acetaldehyde $(0.29 \mathrm{~mL}, 5.11 \mathrm{mmol}, 1.20$ equiv) in tetrahydrofuran $(4 \mathrm{~mL}$ ) was added dropwise over a period of $5 \mathrm{~min}$. After $40 \mathrm{~min}$, TLC analysis indicated incomplete conversion of ketone 11, and therefore a further portion of acetaldehyde $(71.7 \mu \mathrm{L}$, $1.28 \mathrm{mmol}, 0.30$ equiv) in tetrahydrofuran $(0.5 \mathrm{~mL})$ was added dropwise over a period of $2 \mathrm{~min}$. After further $30 \mathrm{~min}$, the mixture was diluted with $\mathrm{pH} 7$ buffer solution $(50 \mathrm{~mL})$, saturated aqueous sodium chloride solution $(20 \mathrm{~mL})$ and diethyl ether $(40 \mathrm{~mL})$. The layers were separated, the aqueous layer was extracted with diethyl ether $(3 \times 30 \mathrm{~mL})$ and the combined organic extracts were dried over sodium sulfate. The combined organic extracts were dried over sodium sulfate, the dried solution was filtered and the filtrate was concentrated to provide the aldol product which was used in the next step without further purification.

A solution of the crude aldol product (assuming $4.26 \mathrm{mmol}$ ) in dimethyl formamide $(8.5 \mathrm{~mL}$ ) was treated sequentially with imidazole $(0.93 \mathrm{~g}, 13.6 \mathrm{mmol}, 3.20$ equiv), 4-dimethylaminopyridine $(52.0 \mathrm{mg}$, $0.43 \mathrm{mmol}, 0.10$ equiv) and tert-butyldimethylchlorosilane $\left(0.90 \mathrm{~g}, 5.96 \mathrm{mmol}, 1.40\right.$ equiv) at $0{ }^{\circ} \mathrm{C}$. After $5 \mathrm{~min}$, the cooling bath was removed and the mixture was allowed to warm to $23^{\circ} \mathrm{C}$. After $13 \mathrm{~h}$, the mixture was diluted with saturated aqueous sodium hydrogen carbonate solution $(40 \mathrm{~mL})$, water $(20 \mathrm{~mL})$ and diethyl ether $(20 \mathrm{~mL})$. The layers were separated, the aqueous layer was extracted with diethyl ether $(3 \times 30 \mathrm{~mL})$ and the combined organic extracts were washed with saturated aqueous sodium chloride solution $(40 \mathrm{~mL})$. The washed solution was dried over sodium sulfate. The dried solution was filtered and the filtrate was concentrated. The residue was purified by flash-column chromatography on silica gel ( $5 \%$ ethyl acetate in hexanes) to provide silyl ether 36 (1.57 g, 80\% over two steps, inconsequential 10:1 d.r. at C-10) as a colourless oil which solidified upon standing.

TLC (5\% ethyl acetate in hexanes): $\mathrm{R}_{f}=0.43$ (CAM). Note: Traces of the minor $\mathrm{C}-10$ diastereomer are visible in the ${ }^{1} \mathrm{H}$ and ${ }^{13} \mathrm{C} N M R$ spectra, but solely the resonances of the major diastereomer are listed below. ${ }^{1} \mathrm{H}$ NMR $\left(800 \mathrm{MHz}, \mathrm{C}_{6} \mathrm{D}_{6}\right) \delta 7.31-7.28(\mathrm{~m}, 2 \mathrm{H}, \mathrm{Ph}), 7.26-7.23(\mathrm{~m}, 2 \mathrm{H}, \mathrm{Ph}), 7.15-7.13(\mathrm{~m}, 1 \mathrm{H}, P h)$, 4.7-4.67 (m, 1H, 10-H), 4.17-4.09 (m, 2H, Bn), 2.75-2.69 (m, 1H, 2- $\left.\mathrm{H}_{\mathrm{A}}\right), 2.66-2.61(\mathrm{~m}, 1 \mathrm{H}, 9-\mathrm{H}), 2.60$ (dd, $\left.{ }^{2} J_{2 \mathrm{~B} / 2 \mathrm{~A}}=15.5 \mathrm{~Hz},{ }^{3} J_{2 \mathrm{~B} / 3}=3.9 \mathrm{~Hz}, 1 \mathrm{H}, 2-\mathrm{H}_{\mathrm{B}}\right), 2.09\left(\mathrm{dd},{ }^{2} \mathrm{~J}_{8 \mathrm{~A} / 8 \mathrm{~B}}=12.6 \mathrm{~Hz},{ }^{3} J_{8 \mathrm{~A} / 9}=6.5 \mathrm{~Hz}, 1 \mathrm{H}, 8-\mathrm{H}_{\mathrm{A}}\right), 2.05-1.99$ $\left(\mathrm{m}, 1 \mathrm{H}, 5-\mathrm{H}_{\mathrm{A}}\right), 1.84\left(\mathrm{~h},{ }^{3} \mathrm{~J}_{15 / 16-17}=6.9 \mathrm{~Hz}, 1 \mathrm{H}, 15-\mathrm{H}\right), 1.67\left(\mathrm{dd},{ }^{3} J_{3 / 2 \mathrm{~A}}=14.3 \mathrm{~Hz},{ }^{3} J_{3 / 2 \mathrm{~B}}=3.9 \mathrm{~Hz}, 1 \mathrm{H}, 3-\mathrm{H}\right)$, 1.57-1.49 (m, 2H, 5- $\left.\mathrm{H}_{\mathrm{B}}, 6-\mathrm{H}_{\mathrm{A}}\right), 1.43\left(\mathrm{app} \mathrm{t},{ }^{2} \mathrm{~J}_{8 \mathrm{~B} / 8 \mathrm{~A}}={ }^{3} \mathrm{~J}_{8 \mathrm{~B} / 9}=12.6 \mathrm{~Hz}, 1 \mathrm{H}, 8-\mathrm{H}_{\mathrm{B}}\right), 1.25\left(\mathrm{~d},{ }^{3} \mathrm{~J}_{11 / 10}=6.2 \mathrm{~Hz}, 3 \mathrm{H}\right.$, $11-\mathrm{H}), 1.11(\mathrm{~s}, 3 \mathrm{H}, 18-\mathrm{H}), 1.08-1.02\left(\mathrm{~m}, 1 \mathrm{H}, 6-\mathrm{H}_{\mathrm{B}}\right), 0.99(\mathrm{~s}, 9 \mathrm{H}, \operatorname{SiR}), 0.78\left(\mathrm{~d},{ }^{3} \mathrm{~J}=6.8 \mathrm{~Hz}, 3 \mathrm{H}, 16-\mathrm{H}, 17-\mathrm{H}\right)$, $0.68\left(\mathrm{~d},{ }^{3} \mathrm{~J}=6.8 \mathrm{~Hz}, 3 \mathrm{H}, 16-\mathrm{H}, 17-\mathrm{H}\right), 0.10(\mathrm{~s}, 6 \mathrm{H}, \mathrm{SiR}) .{ }^{13} \mathrm{C} \mathrm{NMR}\left(200 \mathrm{MHz}, \mathrm{C}_{6} \mathrm{D}_{6}\right) \delta 210.42(\mathrm{C}-1)$, $140.13(P h), 128.57(P h), 127.33(P h), 127.17(P h), 87.58(\mathrm{C}-4), 67.51(\mathrm{C}-10), 62.84(B n), 52.95(\mathrm{C}-9)$, 50.46 (C-3), 41.94 (C-7), 41.90 (C-2), 40.38 (C-6), 38.07 (C-8), 34.86 (C-5), 32.90 (C-15), 26.15 (SiR), 19.85 (C-11), 18.85 (C-18), 18.32 (SiR), 18.10 (C-16, C-17), 17.72 (C-16, C-17), -4.36 (SiR), -4.76 (SiR). IR (Diamond-ATR, neat) $\tilde{v}_{\text {max }}: 2955$ (s), 2930 (s), 2857 (m), 1700 (s), $1471(\mathrm{~m}), 1387$ (m), 1254 (s), 1109 (s), 
1066 (s), 1043 (s), $1006(\mathrm{~m}) \mathrm{cm}^{-1}$. HRMS (EI): calcd for ([M-tBu], $\left.\mathrm{C}_{24} \mathrm{H}_{37} \mathrm{O}_{3} \mathrm{Si}\right)^{+}:$401.2512, found: 401.2516. $[\alpha]_{D}^{20}=+26.4^{\circ}\left(\mathrm{c}=0.33, \mathrm{CH}_{2} \mathrm{Cl}_{2}\right)$. 


\subsection{Synthesis of allylic alcohol 12}
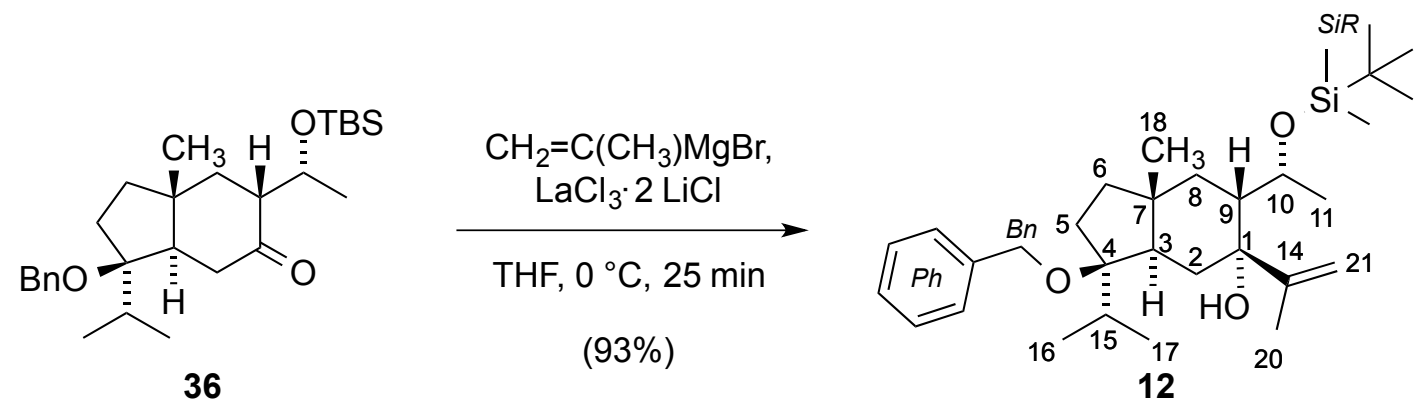

A solution of silyl ether 36 (1.56 g, $3.40 \mathrm{mmol}, 1$ equiv, 10:1 d.r. at C-10) in tetrahydrofuran (3.5 mL) was treated with lanthanum(III) chloride bis(lithium chloride) complex solution (0.46 M in THF, $7.39 \mathrm{~mL}$, $3.40 \mathrm{mmol}, 1.00$ equiv) at $23{ }^{\circ} \mathrm{C}$. After $1 \mathrm{~h}$, the brownish solution was cooled to $0{ }^{\circ} \mathrm{C}$, whereupon isopropenylmagnesium bromide solution ( $0.5 \mathrm{M}$ in THF, $20.4 \mathrm{~mL}, 10.2 \mathrm{mmol}, 3.00$ equiv) was added dropwise over a period of $20 \mathrm{~min}$ (syringe pump). After complete addition, stirring was continued for $5 \mathrm{~min}$, before the mixture was diluted with $\mathrm{pH} 7$ buffer solution $(50 \mathrm{~mL})$, saturated aqueous sodium chloride solution $(20 \mathrm{~mL}$ ) and diethyl ether $(40 \mathrm{~mL})$. The layers were separated, the aqueous layer was extracted with diethyl ether $(5 \times 40 \mathrm{~mL})$ and the combined organic extracts were dried over sodium sulfate. The dried solution was filtered and the filtrate was concentrated. The residue was purified by flash-column chromatography on silica gel (5\% ethyl acetate in hexanes) to provide allylic alcohol $12(1.58 \mathrm{~g}, 93 \%)$ as a colourless oil.

TLC (5\% ethyl acetate in hexanes): $\mathrm{R}_{f}=0.32$ (CAM). ${ }^{1} \mathrm{H}$ NMR $\left(400 \mathrm{MHz}, \mathrm{C}_{6} \mathrm{D}_{6}\right) \delta 7.41-7.37(\mathrm{~m}, 2 \mathrm{H}, P h)$, 7.25-7.19 (m, 2H, Ph), 7.13-7.06 (m, 1H,Ph), $5.50\left(\mathrm{~d},{ }^{2} \mathrm{~J}_{21 \mathrm{~A} / 21 \mathrm{~B}}=2.2 \mathrm{~Hz}, 1 \mathrm{H}, 21-\mathrm{H}_{\mathrm{A}}\right), 4.93-4.88(\mathrm{~m}, 1 \mathrm{H}$, $\left.21-\mathrm{H}_{\mathrm{B}}\right), 4.33-4.22(\mathrm{~m}, 2 \mathrm{H}, \mathrm{Bn}), 3.81-3.72(\mathrm{~m}, 1 \mathrm{H}, 10-\mathrm{H}), 3.59\left(\mathrm{~d},{ }^{4} \mathrm{~J}_{1 \mathrm{OH} / 2 \mathrm{~A}}=1.3 \mathrm{~Hz}, 1 \mathrm{H}, 1-\mathrm{OH}\right), 2.32-2.19(\mathrm{~m}$, $\left.2 \mathrm{H}, 2-\mathrm{H}_{\mathrm{A}}, 3-\mathrm{H}\right), 2.16-2.00\left(\mathrm{~m}, 2 \mathrm{H}, 5-\mathrm{H}_{\mathrm{A}}, 15-\mathrm{H}\right), 1.98-1.88\left(\mathrm{~m}, 2 \mathrm{H}, 8-\mathrm{H}_{\mathrm{A}}, 9-\mathrm{H}\right), 1.75(\mathrm{~s}, 3 \mathrm{H}, 20-\mathrm{H}), 1.72-1.61$ $\left(\mathrm{m}, 3 \mathrm{H}, 2-\mathrm{H}_{\mathrm{B}}, 5-\mathrm{H}_{\mathrm{B}}, 6-\mathrm{H}_{\mathrm{A}}\right), 1.53\left(\mathrm{~d},{ }^{2} \mathrm{~J}_{8 \mathrm{~B} / 8 \mathrm{~A}}=8.5 \mathrm{~Hz}, 1 \mathrm{H}, 8-\mathrm{H}_{\mathrm{B}}\right), 1.34-1.24\left(\mathrm{~m}, 4 \mathrm{H}, 6-\mathrm{H}_{\mathrm{B}}, 11-\mathrm{H}\right), 1.21(\mathrm{~s}, 3 \mathrm{H}$, $18-\mathrm{H}), 1.01\left(\mathrm{~d},{ }^{3} \mathrm{~J}=6.8 \mathrm{~Hz}, 3 \mathrm{H}, 16-\mathrm{H}, 17-\mathrm{H}\right), 0.99-0.92(\mathrm{~m}, 12 \mathrm{H}, 16-\mathrm{H}, 17-\mathrm{H}, \mathrm{SiR}), 0.04(\mathrm{~s}, 3 \mathrm{H}, \mathrm{SiR}), 0.00(\mathrm{~s}$, 3H, SiR). ${ }^{13} \mathrm{C}$ NMR (100 MHz, $\left.\mathrm{C}_{6} \mathrm{D}_{6}\right) \delta 153.45(\mathrm{C}-14), 140.70(P h), 128.52(P h), 127.33(P h), 127.14(P h)$, 110.27 (C-21), 88.71 (C-4), 78.59 (C-1), 74.41 (C-10), 63.20 (Bn), 45.17 (C-3), 42.50 (C-9), 42.28 (C-7), 41.66 (C-8), 41.34 (C-6), 38.01 (C-2), 34.25 (C-5), 33.19 (C-15), 25.99 (SiR), 22.55 (C-11), 20.24 (C-20), 19.11 (C-18), 18.53 (C-16, C-17), 18.34 (C-16, C-17), 18.13 (SiR), -4.11 (SiR), -4.87 (SiR). IR (Diamond-ATR, neat) $\tilde{v}_{\text {max }} 3461(\mathrm{br}), 2954(\mathrm{~s}), 2930(\mathrm{~s}), 2858(\mathrm{~m}), 1454(\mathrm{~m}), 1386(\mathrm{~s}), 1254(\mathrm{~s}), 1118(\mathrm{~m}), 1060(\mathrm{~s}), 1028$ (m) $\mathrm{cm}^{-1}$. HRMS (ESI): calcd for $\left([\mathrm{M}+\mathrm{H}], \mathrm{C}_{31} \mathrm{H}_{53} \mathrm{O}_{3} \mathrm{Si}\right)^{+}:$501.37640, found: 501.37617. $[\boldsymbol{\alpha}]_{\boldsymbol{D}}^{\mathbf{2 0}}=+20.0^{\circ}$ (c $=0.20, \mathrm{CH}_{2} \mathrm{Cl}_{2}$ ). 


\subsection{Synthesis of $\beta$-hydroxyketone 13}

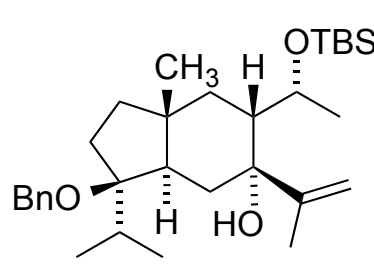

12
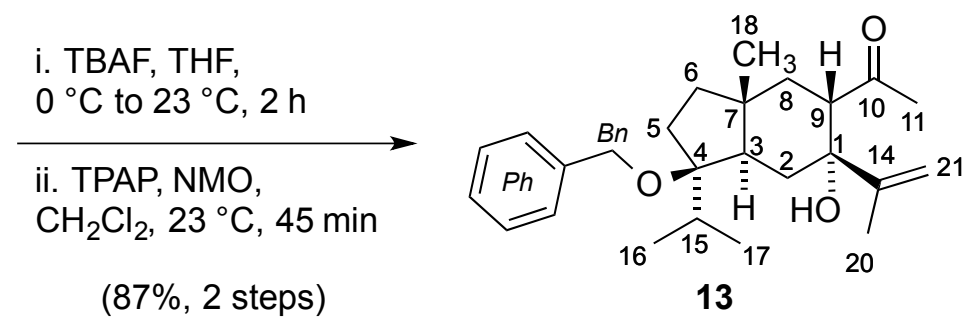

A solution of allylic alcohol 12 (1.57 g, $3.13 \mathrm{mmol}, 1$ equiv) in tetrahydrofuran ( $30 \mathrm{~mL}$ ) was treated with tetrabutylammonium fluoride solution ( $1 \mathrm{M}$ in THF, $4.70 \mathrm{~mL}, 4.70 \mathrm{mmol}, 1.50$ equiv) at $0{ }^{\circ} \mathrm{C}$. After $1.5 \mathrm{~h}$, the cooling bath was removed and the mixture was allowed to warm to $23^{\circ} \mathrm{C}$. After $30 \mathrm{~min}$, the mixture was diluted with saturated aqueous sodium hydrogen carbonate solution $(80 \mathrm{~mL})$ and diethyl ether $(20 \mathrm{~mL})$. The layers were separated, the aqueous layer was extracted with diethyl ether $(3 \times 40 \mathrm{~mL})$ and the combined organic extracts were dried over sodium sulfate. The dried solution was filtered and the filtrate was concentrated to provide the crude diol which was used in the next step without further purification.

A solution of the crude diol (assuming $3.13 \mathrm{mmol}$ ) in dichloromethane $(24 \mathrm{~mL}$ ) was treated sequentially with $4 \AA$ molecular sieves $(600 \mathrm{mg})$, 4-methylmorpholine $\mathrm{N}$-oxide $(0.55 \mathrm{~g}, 4.70 \mathrm{mmol}, 1.50$ equiv) and tetrapropylammonium perruthenate $\left(77.0 \mathrm{mg}, 0.21 \mathrm{mmol}, 0.07\right.$ equiv) at $23^{\circ} \mathrm{C}$. After $45 \mathrm{~min}$, the mixture was concentrated to a small volume and the black oily residue was purified by flash-column chromatography on silica gel (10\% ethyl acetate in hexanes) to provide $\beta$-hydroxyketone 13 (1.05 g, $87 \%$ over two steps) as a yellowish oil which solidified upon standing.

TLC (10\% ethyl acetate in hexanes): $\mathrm{R}_{f}=0.33$ (CAM). ${ }^{1} \mathrm{H}$ NMR $\left(400 \mathrm{MHz}, \mathrm{C}_{6} \mathrm{D}_{6}\right) \delta 7.38-7.33(\mathrm{~m}, 2 \mathrm{H}, \mathrm{Ph})$, 7.24-7.18 (m, 2H, Ph), 7.13-7.06 (m, 1H, Ph), 5.21-5.18 (m, 1H, 21- $\left.\mathrm{H}_{\mathrm{A}}\right), 4.91(\mathrm{br} \mathrm{s}, 1 \mathrm{H}, 1-\mathrm{OH}), 4.83-4.80$ $\left(\mathrm{m}, 1 \mathrm{H}, 21-\mathrm{H}_{\mathrm{B}}\right), 4.30-4.20(\mathrm{~m}, 2 \mathrm{H}, \mathrm{Bn}), 2.97\left(\mathrm{dd},{ }^{3} J_{9 / 8 \mathrm{~A}}=12.7 \mathrm{~Hz},{ }^{3} \mathrm{~J}_{9 / 8 \mathrm{~B}}=3.8 \mathrm{~Hz}, 1 \mathrm{H}, 9-\mathrm{H}\right), 2.43-2.35(\mathrm{~m}, 1 \mathrm{H}$, 3-H), 2.09-1.99 (m, 2H, 5- $\left.\mathrm{H}_{\mathrm{A}}, 15-\mathrm{H}\right), 1.98-1.93(\mathrm{~m}, 2 \mathrm{H}, 2-\mathrm{H}), 1.69-1.67(\mathrm{~m}, 3 \mathrm{H}, 20-\mathrm{H}), 1.67-1.57(\mathrm{~m}, 5 \mathrm{H}$, $\left.5-\mathrm{H}_{\mathrm{B}}, 8-\mathrm{H}_{\mathrm{A}}, 11-\mathrm{H}\right), 1.50\left(\mathrm{dd},{ }^{2} J_{6 \mathrm{~A} / 6 \mathrm{~B}}=11.6 \mathrm{~Hz},{ }^{3} J_{6 \mathrm{~A} / 5 \mathrm{~A}}=7.9 \mathrm{~Hz}, 1 \mathrm{H}, 6-\mathrm{H}_{\mathrm{A}}\right), 1.38\left(\mathrm{dd},{ }^{2} J_{8 \mathrm{~B} / 8 \mathrm{~A}}=12.2 \mathrm{~Hz}\right.$, $\left.{ }^{3} J_{8 \mathrm{~B} / 9}=3.8 \mathrm{~Hz}, 1 \mathrm{H}, 8-\mathrm{H}_{\mathrm{B}}\right), 1.18-1.08\left(\mathrm{~m}, 1 \mathrm{H}, 6-\mathrm{H}_{\mathrm{B}}\right), 1.07(\mathrm{~s}, 3 \mathrm{H}, 18-\mathrm{H}), 1.05\left(\mathrm{~d},{ }^{3} \mathrm{~J}=6.8 \mathrm{~Hz}, 3 \mathrm{H}, 16-\mathrm{H}, 17-\mathrm{H}\right)$, $0.98\left(\mathrm{~d},{ }^{3} \mathrm{~J}=6.8 \mathrm{~Hz}, 3 \mathrm{H}, 16-\mathrm{H}, 17-\mathrm{H}\right) .{ }^{13} \mathrm{C}$ NMR $\left(100 \mathrm{MHz}, \mathrm{C}_{6} \mathrm{D}_{6}\right) \delta 215.29(\mathrm{C}-10), 152.16(\mathrm{C}-14), 140.91(P h)$, $128.91(P h), 127.72(P h), 127.58(P h), 110.84$ (C-21), 88.90 (C-4), 77.24 (C-1), 63.56 (Bn), 50.80 (C-9), 45.54 (C-3), 42.21 (C-7), 40.81 (C-6), 40.34 (C-8), 36.02 (C-2), 34.50 (C-5), 33.47 (C-15), 30.69 (C-11), 20.15 (C-20), 19.18 (C-18), 18.87 (C-16, C-17), 18.62 (C-16, C-17). IR (Diamond-ATR, neat) $\tilde{v}_{\max }: 3462$ (br), 2958 (s), 1695 (s), 1639 (w), 1453 (s), $1386(\mathrm{~s}), 1182(\mathrm{~m}), 1163(\mathrm{~m}), 1086(\mathrm{~m}), 1058(\mathrm{~s}), 1027(\mathrm{~s}) \mathrm{cm}^{-1}$. HRMS (ESI): calcd for ([M+CH $\left.\left.\mathrm{CH}_{3} \mathrm{COO}\right], \mathrm{C}_{27} \mathrm{H}_{39} \mathrm{O}_{5}\right)^{-}: 443.27975$, found: 443.28067 . $[\alpha]_{D}^{20}=+15.6^{\circ}(\mathrm{c}=0.50$, $\mathrm{CH}_{2} \mathrm{Cl}_{2}$ ). 


\subsection{Synthesis of diene 37}

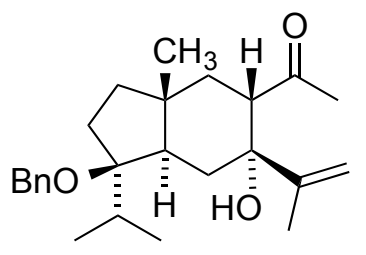

13

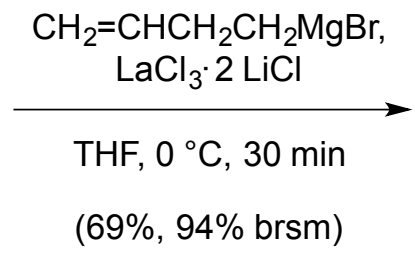

$(69 \%, 94 \%$ brsm $)$

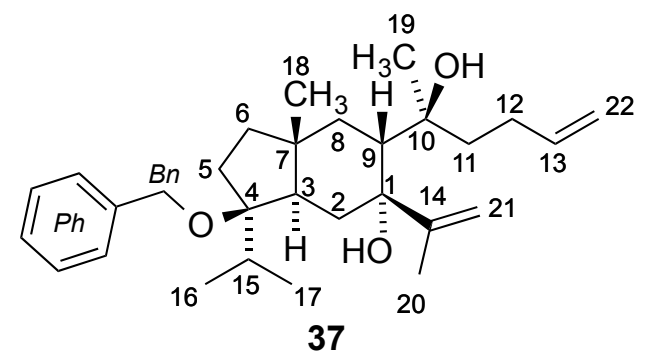

Grignard stock solution preparation: A suspension of magnesium turnings ( $0.32 \mathrm{~g}, 13.0 \mathrm{mmol}, 1$ equiv) in tetrahydrofuran $(8 \mathrm{~mL})$ was treated dropwise with a solution of 4-bromo-1-butene $(1.32 \mathrm{~mL}, 13.0 \mathrm{mmol}$, 1.00 equiv) in tetrahydrofuran $(5 \mathrm{~mL})$ over a period of $10 \mathrm{~min}$ so as to maintain a gentle reflux. After complete addition, stirring was continued at $23{ }^{\circ} \mathrm{C}$ for $1 \mathrm{~h}$, before the mixture was filtered (argon atmosphere) and used immediately in the following reaction.

In a separate flask, a solution of $\beta$-hydroxyketone $13(1.00 \mathrm{~g}, 2.60 \mathrm{mmol}, 1$ equiv) in tetrahydrofuran $(3 \mathrm{~mL}$ ) was treated with lanthanum(III) chloride bis(lithium chloride) complex solution (0.46 M in THF, $5.65 \mathrm{~mL}, 2.60 \mathrm{mmol}, 1.00$ equiv) at $23^{\circ} \mathrm{C}$. After $1 \mathrm{~h}$, the brownish solution was cooled to $0{ }^{\circ} \mathrm{C}$, whereupon freshly prepared 3-butenylmagnesium bromide solution (assuming $0.70 \mathrm{M}$ in THF, $11.1 \mathrm{~mL}, 7.80 \mathrm{mmol}$, 3.00 equiv) was added dropwise over a period of $20 \mathrm{~min}$ (syringe pump). After end of the addition, stirring was continued for $10 \mathrm{~min}$, before the mixture was diluted with $\mathrm{pH} 7$ buffer solution $(70 \mathrm{~mL})$, saturated aqueous sodium chloride solution $(30 \mathrm{~mL})$ and diethyl ether $(40 \mathrm{~mL})$. The layers were separated, the aqueous layer was extracted with diethyl ether $(5 \times 40 \mathrm{~mL})$ and the combined organic extracts were dried over sodium sulfate. The dried solution was filtered and the filtrate was concentrated. The residue was purified by flash-column chromatography on silica gel $(15 \% \rightarrow 20 \%$ ethyl acetate in hexanes) to provide recovered $\beta$-hydroxyketone $13(0.27 \mathrm{~g}, 27 \%)$ as a yellowish oil and diene $37(0.79 \mathrm{~g}, 69 \%, 94 \% \mathrm{brsm})$ as a yellowish foam.

TLC (10\% ethyl acetate in hexanes): $\mathrm{R}_{f}=0.20$ (CAM). Note: diene 37 shows signal broadening in both the ${ }^{1} \mathrm{H}$ and ${ }^{13} \mathrm{C}$ NMR spectra due to hindered rotation of the sidechains. ${ }^{1} \mathrm{H}$ NMR $\left(400 \mathrm{MHz}, \mathrm{C}_{6} \mathrm{D}_{6}\right) \delta 7.39-7.34$ $(\mathrm{m}, 2 \mathrm{H}, \mathrm{Ph}), 7.25-7.18(\mathrm{~m}, 2 \mathrm{H}, \mathrm{Ph}), 7.14-7.07(\mathrm{~m}, 1 \mathrm{H}, \mathrm{Ph}), 5.78-5.56\left(\mathrm{~m}, 2 \mathrm{H}, 13-\mathrm{H}, 21-\mathrm{H}_{\mathrm{A}}\right), 5.08-4.90(\mathrm{~m}$, $2 \mathrm{H}, 22-\mathrm{H}), 4.84\left(\mathrm{br} \mathrm{s}, 1 \mathrm{H}, 21-\mathrm{H}_{\mathrm{B}}\right), 4.34-4.19(\mathrm{~m}, 2 \mathrm{H}, \mathrm{Bn}), 4.13$ (br s, 1H, 1-OH, 10-OH), 2.34-2.13 (m, 2H, $\left.2-\mathrm{H}_{\mathrm{A}}, 3-\mathrm{H}\right), 2.13-1.93\left(\mathrm{~m}, 3 \mathrm{H}, 5-\mathrm{H}_{\mathrm{A}}, 9-\mathrm{H}, 15-\mathrm{H}\right), 1.93-1.83(\mathrm{~m}, 2 \mathrm{H}, 12-\mathrm{H}), 1.78-1.51\left(\mathrm{~m}, 9 \mathrm{H}, 2-\mathrm{H}_{B}, 5-\mathrm{H}_{B}\right.$, $\left.6-\mathrm{H}_{\mathrm{A}}, 8-\mathrm{H}, 11-\mathrm{H}_{\mathrm{A}}, 20-\mathrm{H}\right), 1.34-1.18\left(\mathrm{~m}, 5 \mathrm{H}, 6-\mathrm{H}_{B}, 11-\mathrm{H}_{\mathrm{B}}, 19-\mathrm{H}\right), 1.11$ (br s, 3H, 18-H), 1.02 (br d, ${ }^{3} \mathrm{~J}=6.8 \mathrm{~Hz}$, $3 \mathrm{H}, 16-\mathrm{H}, 17-\mathrm{H}), 0.98\left(\mathrm{~d},{ }^{3} \mathrm{~J}=6.8 \mathrm{~Hz}, 3 \mathrm{H}, 16-\mathrm{H}, 17-\mathrm{H}\right) .{ }^{13} \mathrm{C} N M R\left(100 \mathrm{MHz}, \mathrm{C}_{6} \mathrm{D}_{6}\right) \delta 155.66(\mathrm{C}-14)$, $140.66(P h), 138.78(\mathrm{C}-13), 128.50(P h), 127.27(P h), 127.13(P h), 114.63(\mathrm{C}-22), 109.81(\mathrm{C}-21)$, 88.78 (C-4), 79.82 (C-1), 77.52 (C-10), 63.17 (Bn), 44.92 (C-3), 42.41 (C-6, C-7, C-9, C-11), 42.18 (C-6, C-7, C-9, C-11), 41.35 (C-6, C-7, C-9, C-11), 41.18 (C-6, C-7, C-9, C-11), 39.48 (C-2), 38.18 (C-8), 34.20 (C-5), 33.23 (C-15), 29.18 (C-12), 26.47 (C-19), 20.59 (C-20), 19.02 (C-18), 18.55 (C-16, C-17), 18.30 (C-16, C-17). IR (Diamond-ATR, neat) $\tilde{v}_{\text {max }} 3366$ (br), 2944 (s), 2873 (s), 1639 (m), 1453 (s), 1385 (s), 1345 (m), $1142(\mathrm{~m}), 1059(\mathrm{~s}), 906$ (s) $\mathrm{cm}^{-1}$. HRMS (ESI): calcd for ([M+ $\left.\left.\mathrm{CH}_{3} \mathrm{COO}\right], \mathrm{C}_{31} \mathrm{H}_{47} \mathrm{O}_{5}\right)^{-}:$499.34235, found: 499.34308. $[\alpha]_{D}^{20}=+11.3^{\circ}\left(\mathrm{c}=0.30, \mathrm{CH}_{2} \mathrm{Cl}_{2}\right)$. 


\subsection{Synthesis of tricycle 14}

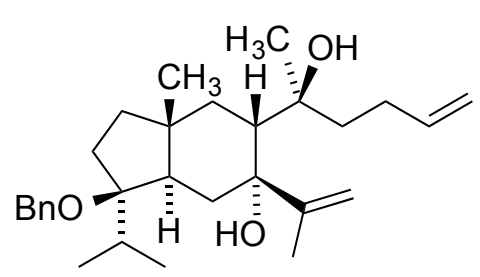

37

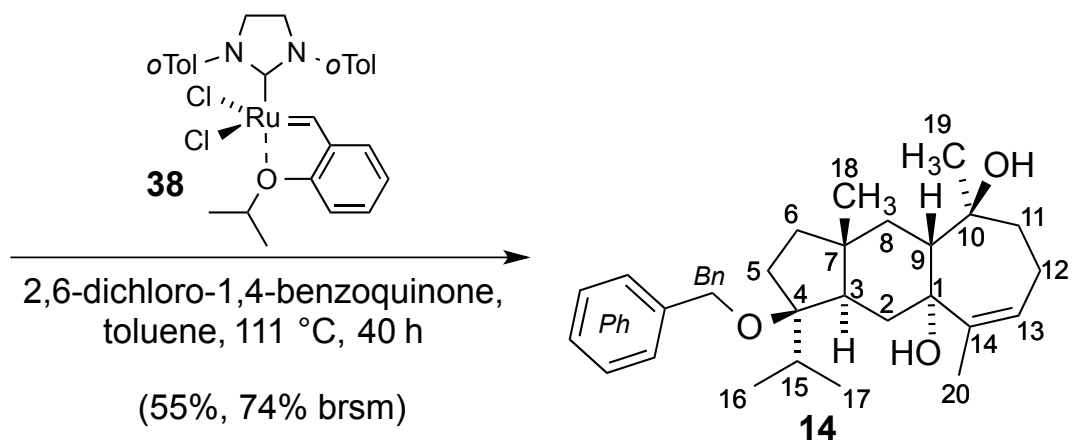

A solution of diene 37 ( $0.42 \mathrm{~g}, 0.95 \mathrm{mmol}, 1$ equiv) in degassed (sparged with argon for 60 min prior to use) toluene $(900 \mathrm{~mL}$ ) was treated with 2,6,-dichloro-1-4-benzoquinone ( $33.7 \mathrm{mg}, 0.19 \mathrm{mmol}, 0.20$ equiv) and Stewart-Grubbs catalyst 38 (109.0 $\mathrm{mg}, 0.19 \mathrm{mmol}, 0.20$ equiv). The resulting orange mixture was then heated to $111^{\circ} \mathrm{C}$, whereupon it turned dark brown. After $24 \mathrm{~h}$, a further portion of Stewart-Grubbs catalyst $38\left(27.2 \mathrm{mg}, 47.7 \mu \mathrm{mol}, 0.05\right.$ equiv) was added and stirring was continued at $111{ }^{\circ} \mathrm{C}$. After further $16 \mathrm{~h}$, the mixture was allowed to cool to $23^{\circ} \mathrm{C}$ and then was concentrated in vacuo. The residue was purified by flash-column chromatography on silica gel $(25 \% \rightarrow 30 \%$ ethyl acetate in hexanes) to provide recovered diene 37 (106 mg, 25\%) as a yellow oil and tricycle $14(0.22 \mathrm{~g}, 55 \%, 74 \% \mathrm{brsm})$ as an off-white foam.

TLC (20\% ethyl acetate in hexanes): $\mathrm{R}_{f}=0.15$ (CAM). ${ }^{1} \mathrm{H}$ NMR $\left(800 \mathrm{MHz}, \mathrm{C}_{6} \mathrm{D}_{6}\right) \delta 7.38-7.32(\mathrm{~m}, 2 \mathrm{H}, P h)$, 7.22-7.18 $(\mathrm{m}, 2 \mathrm{H}, P h), 7.11-7.07(\mathrm{~m}, 1 \mathrm{H}, \mathrm{Ph}), 5.60-5.56(\mathrm{~m}, 1 \mathrm{H}, 13-\mathrm{H}), 4.30-4.23(\mathrm{~m}, 2 \mathrm{H}, B n)$, $2.37-2.30\left(\mathrm{~m}, 1 \mathrm{H}, 12-\mathrm{H}_{\mathrm{A}}\right), 2.24\left(\mathrm{dd},{ }^{3} J_{9 / 8 \mathrm{~B}}=12.6 \mathrm{~Hz},{ }^{3} J_{9 / 8 \mathrm{~A}}=3.8 \mathrm{~Hz}, 1 \mathrm{H}, 9-\mathrm{H}\right), 2.16\left(\mathrm{~d},{ }^{2} \mathrm{~J}_{2 \mathrm{~A} / 2 \mathrm{~B}}=12.5 \mathrm{~Hz}, 1 \mathrm{H}\right.$, $\left.2-\mathrm{H}_{\mathrm{A}}\right), 2.08-2.01\left(\mathrm{~m}, 3 \mathrm{H}, 5-\mathrm{H}_{\mathrm{A}}, 8-\mathrm{H}_{\mathrm{A}}, 15-\mathrm{H}\right), 1.93-1.85\left(\mathrm{~m}, 2 \mathrm{H}, 2-\mathrm{H}_{B}, 3-\mathrm{H}\right), 1.74-1.57\left(\mathrm{~m}, 9 \mathrm{H}, 5-\mathrm{H}_{B}, 6-\mathrm{H}_{\mathrm{A}}\right.$, $\left.8-\mathrm{H}_{\mathrm{B}}, 11-\mathrm{H}, 12-\mathrm{H}_{\mathrm{B}}, 20-\mathrm{H}\right), 1.27(\mathrm{~s}, 3 \mathrm{H}, 19-\mathrm{H}), 1.22-1.17\left(\mathrm{~m}, 1 \mathrm{H}, 6-\mathrm{H}_{\mathrm{B}}\right), 1.13(\mathrm{~s}, 3 \mathrm{H}, 18-\mathrm{H}), 1.03(\mathrm{~d}$, $\left.{ }^{3} \mathrm{~J}=6.8 \mathrm{~Hz}, 3 \mathrm{H}, 16-\mathrm{H}, 17-\mathrm{H}\right), 0.93\left(\mathrm{~d},{ }^{3} \mathrm{~J}=6.8 \mathrm{~Hz}, 3 \mathrm{H}, 16-\mathrm{H}, 17-\mathrm{H}\right), 0.82(\mathrm{br} \mathrm{s}, 1 \mathrm{H}, 1-\mathrm{OH}), 0.75$ (br s, $1 \mathrm{H}$, 10-OH). ${ }^{13} \mathrm{C}$ NMR $\left(200 \mathrm{MHz}, \mathrm{C}_{6} \mathrm{D}_{6}\right) \delta 141.17(\mathrm{C}-14), 140.59(P h), 131.05(\mathrm{C}-13), 128.52(P h), 127.16(P h)$, $127.11(P h), 88.69$ (C-4), 76.60 (C-1), 74.78 (C-10), 63.08 (Bn), 46.53 (C-9), 46.08 (C-11), 45.88 (C-3), 41.56 (C-7), 41.42 (C-6), 39.52 (C-8), 38.25 (C-2), 34.26 (C-5), 33.22 (C-15), 28.12 (C-19), 22.28 (C-12), 21.39 (C-20), 18.61 (C-16, C-17), 18.53 (C-18), 18.36 (C-16, C-17). IR (Diamond-ATR, neat) $\tilde{v}_{\max }: 3462$ (br), $2955(\mathrm{~s}), 2873(\mathrm{~m}), 1453(\mathrm{~m}), 1386(\mathrm{~m}), 1306(\mathrm{~m}), 1090(\mathrm{~m}), 1063(\mathrm{~m}) \mathrm{cm}^{-1}$. HRMS (ESI): calcd for $\left(\left[\mathrm{M}+\mathrm{CH}_{3} \mathrm{COO}\right], \mathrm{C}_{29} \mathrm{H}_{43} \mathrm{O}_{5}\right)^{-}: 471.31105$, found: 471.31184. $[\alpha]_{D}^{20}=+17.3^{\circ}\left(\mathrm{c}=0.87, \mathrm{CH}_{2} \mathrm{Cl}_{2}\right.$ ). 


\subsection{Synthesis of epoxide 15}
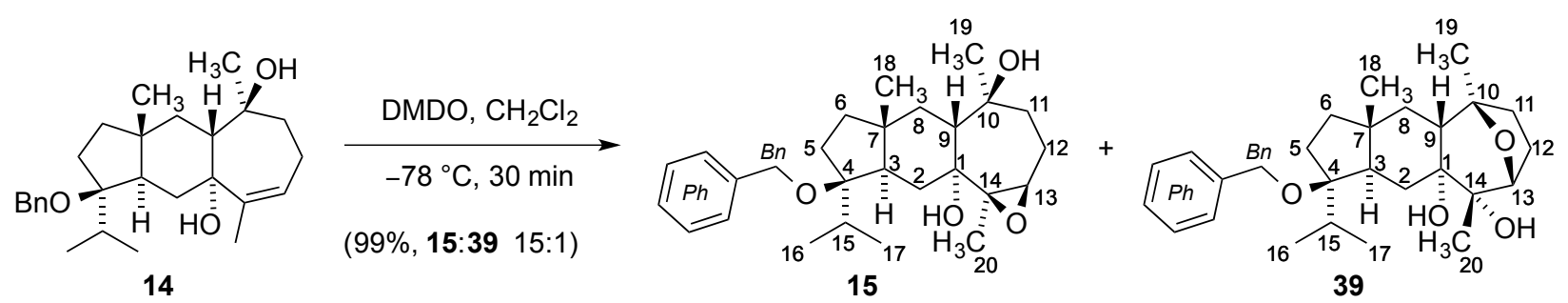

A solution of tricycle 14 (100 mg, $0.24 \mathrm{mmol}, 1$ equiv) in dichloromethane $(4 \mathrm{~mL}$ ) was treated dropwise over a period of $5 \mathrm{~min}$ with freshly prepared dimethyldioxirane solution $(0.064 \mathrm{M}$ in acetone, $5.68 \mathrm{~mL}$, $0.36 \mathrm{mmol}, 1.50$ equiv) at $-78{ }^{\circ} \mathrm{C}$. After $15 \mathrm{~min}$, more dimethyldioxirane solution ( $0.064 \mathrm{M}$ in acetone, $5.68 \mathrm{~mL}, 0.36 \mathrm{mmol}, 1.50$ equiv) was added. After $15 \mathrm{~min}$, the mixture was allowed to warm to $23^{\circ} \mathrm{C}$, and then was concentrated. Residual water was removed by azeotropic distillation using benzene

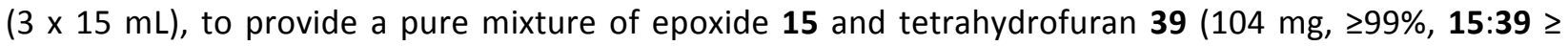
$15: 1)^{8}$ as an off-white foam which was used in the next step without further purification.

A small quantity of this mixture was purified by flash-column chromatography on silica gel (30\% ethyl acetate in hexanes) to provide analytically pure samples of epoxide $\mathbf{1 5}$ and tetrahydrofuran $\mathbf{3 9}$.

Epoxide 15: TLC (30\% ethyl acetate in hexanes): $\mathrm{R}_{f}=0.16$ (CAM). ${ }^{1} \mathbf{H}$ NMR $\left(800 \mathrm{MHz}, \mathrm{C}_{6} \mathrm{D}_{6}\right) \delta 7.37-7.34$ $(\mathrm{m}, 2 \mathrm{H}, P h), 7.15-7.13(\mathrm{~m}, 2 \mathrm{H}, P h), 7.07-7.03(\mathrm{~m}, 1 \mathrm{H}, P h), 4.31-4.24(\mathrm{~m}, 2 \mathrm{H}, \mathrm{Bn}), 2.90$ (dd, $\left.{ }^{3} J_{13 / 12 \mathrm{~A}}=8.4 \mathrm{~Hz},{ }^{3} J_{13 / 12 \mathrm{~B}}=3.9 \mathrm{~Hz}, 1 \mathrm{H}, 13-\mathrm{H}\right), 2.49\left(\mathrm{app} \mathrm{t},{ }^{2} J_{2 \mathrm{~A} / 2 \mathrm{~B}}={ }^{3} J_{2 \mathrm{~A} / 3}=14.1 \mathrm{~Hz}, 1 \mathrm{H}, 2-\mathrm{H}_{\mathrm{A}}\right), 2.12(\mathrm{dd}$, $\left.{ }^{3} J_{9 / 8 B}=12.7 \mathrm{~Hz},{ }^{3} J_{9 / 8 \mathrm{~A}}=4.3 \mathrm{~Hz}, 1 \mathrm{H}, 9-\mathrm{H}\right), 2.06-1.99\left(\mathrm{~m}, 4 \mathrm{H}, 2-\mathrm{H}_{B}, 5-\mathrm{H}_{\mathrm{A}}, 8-\mathrm{H}_{\mathrm{A}}, 15-\mathrm{H}\right), 1.95-1.89(\mathrm{~m}, 1 \mathrm{H}$, $\left.12-\mathrm{H}_{\mathrm{A}}\right), 1.72-1.67\left(\mathrm{~m}, 1 \mathrm{H}, 11-\mathrm{H}_{\mathrm{A}}\right), 1.66-1.54\left(\mathrm{~m}, 4 \mathrm{H}, 3-\mathrm{H}, 5-\mathrm{H}_{B}, 6-\mathrm{H}_{\mathrm{A}}, 12-\mathrm{H}_{B}\right), 1.46$ (app $\mathrm{t}$, $\left.{ }^{2} J_{8 \mathrm{~B} / 8 \mathrm{~A}}={ }^{3} J_{8 \mathrm{~B} / 9}=12.7 \mathrm{~Hz}, 1 \mathrm{H}, 8-\mathrm{H}_{\mathrm{B}}\right), 1.40-1.36\left(\mathrm{~m}, 1 \mathrm{H}, 11-\mathrm{H}_{\mathrm{B}}\right), 1.23(\mathrm{~s}, 3 \mathrm{H}, 19-\mathrm{H}), 1.17(\mathrm{~s}, 6 \mathrm{H}, 18-\mathrm{H}, 20-\mathrm{H})$, $1.16-1.11\left(\mathrm{~m}, 1 \mathrm{H}, 6-\mathrm{H}_{\mathrm{B}}\right), 0.97\left(\mathrm{~d},{ }^{3} \mathrm{~J}=6.8 \mathrm{~Hz}, 3 \mathrm{H}, 16-\mathrm{H}, 17-\mathrm{H}\right), 0.88\left(\mathrm{~d},{ }^{3} \mathrm{~J}=6.8 \mathrm{~Hz}, 3 \mathrm{H}, 16-\mathrm{H}, 17-\mathrm{H}\right)$. ${ }^{13} \mathrm{C}$ NMR $\left(100 \mathrm{MHz}, \mathrm{C}_{6} \mathrm{D}_{6}\right) \delta 140.37(P h), 128.55(P h), 127.08(P h), 126.93(P h), 88.51(\mathrm{C}-4), 77.36(\mathrm{C}-1)$, 74.75 (C-10), 69.25 (C-13), 63.88 (C-14), 63.15 (Bn), 45.72 (C-3), 41.83 (C-7), 41.37 (C-11), 41.21 (C-6), 41.14 (C-9), 38.89 (C-8), 36.47 (C-2), 34.25 (C-5), 33.26 (C-15), 27.52 (C-19), 21.74 (C-12), 19.50 (C-18, C-20), 18.60 (C-16, C-17), 18.44 (C-16, C-17), 18.23 (C-18, C-20). IR (Diamond-ATR, neat) $\tilde{v}_{\text {max }} 3484$ (br), $2943(\mathrm{~m}), 2876(\mathrm{~m}), 1456(\mathrm{~s}), 1387(\mathrm{~s}), 1201(\mathrm{~m}), 1139(\mathrm{~m}), 1088(\mathrm{~m}), 1062(\mathrm{~s}), 1002(\mathrm{~s}) \mathrm{cm}^{-1}$. HRMS (ESI): calcd for $\left(\left[\mathrm{M}+\mathrm{CH}_{3} \mathrm{COO}\right], \mathrm{C}_{29} \mathrm{H}_{43} \mathrm{O}_{6}\right)^{-}: 487.30596$, found: 487.30672 . $[\alpha]_{D}^{20}=-16.6^{\circ}\left(\mathrm{c}=0.18, \mathrm{CH}_{2} \mathrm{Cl}_{2}\right)$.

Tetrahyodrofuran 39: TLC (30\% ethyl acetate in hexanes): $\mathrm{R}_{f}=0.42$ (CAM). ${ }^{1} \mathbf{H}$ NMR (800 MHz, $\mathrm{C}_{6} \mathrm{D}_{6}$ ) 6 7.40-7.36 (m, 2H, Ph), 7.25-7.22 (m, 2H, Ph), 7.12-7.08 (m, 1H,Ph), 4.31-4.27 (m, 2H, Bn), 3.73 $\left(\mathrm{d},{ }^{3} J_{13 / 12 \mathrm{~B}}=7.6 \mathrm{~Hz}, 1 \mathrm{H}, 13-\mathrm{H}\right), 2.56-2.51\left(\mathrm{~m}, 2 \mathrm{H}, 11-\mathrm{H}_{\mathrm{A}}, 14-\mathrm{OH}\right), 2.44-2.38\left(\mathrm{~m}, 1 \mathrm{H}, 12-\mathrm{H}_{\mathrm{A}}\right), 2.33$ (dd, $\left.{ }^{3} J_{3 / 2 \mathrm{~A}}=12.4 \mathrm{~Hz}, 3_{3 / 2 \mathrm{~B}}^{3}=2.8 \mathrm{~Hz}, 1 \mathrm{H}, 3-\mathrm{H}\right), 2.11-2.03\left(\mathrm{~m}, 2 \mathrm{H}, 5-\mathrm{H}_{\mathrm{A}}, 15-\mathrm{H}\right), 1.87$ (dd, ${ }^{3} J_{9 / 8 \mathrm{~A}}=13.1 \mathrm{~Hz}$, $\left.{ }^{3} J_{9 / 8 B}=3.2 \mathrm{~Hz}, 1 \mathrm{H}, 9-\mathrm{H}\right), 1.86-1.80\left(\mathrm{~m}, 1 \mathrm{H}, 12-\mathrm{H}_{B}\right), 1.69-1.63\left(\mathrm{~m}, 2 \mathrm{H}, 2-\mathrm{H}_{\mathrm{A}}, 5-\mathrm{H}_{\mathrm{B}}\right), 1.60-1.53\left(\mathrm{~m}, 3 \mathrm{H}, 2-\mathrm{H}_{B}\right.$, $\left.6-\mathrm{H}_{\mathrm{A}}, 8-\mathrm{H}_{\mathrm{A}}\right), 1.40-1.30\left(\mathrm{~m}, 2 \mathrm{H}, 8-\mathrm{H}_{\mathrm{B}}, 11-\mathrm{H}_{\mathrm{B}}\right), 1.27(\mathrm{~s}, 3 \mathrm{H}, 19-\mathrm{H}), 1.25-1.19\left(\mathrm{~m}, 1 \mathrm{H}, 6-\mathrm{H}_{\mathrm{B}}\right), 1.12(\mathrm{~s}, 3 \mathrm{H}, 18-\mathrm{H})$, $1.10(\mathrm{~s}, 3 \mathrm{H}, 20-\mathrm{H}), 1.01\left(\mathrm{~d},{ }^{3} \mathrm{~J}=6.8 \mathrm{~Hz}, 3 \mathrm{H}, 16-\mathrm{H}, 17-\mathrm{H}\right), 0.98\left(\mathrm{~d},{ }^{3} \mathrm{~J}=6.8 \mathrm{~Hz}, 3 \mathrm{H}, 16-\mathrm{H}, 17-\mathrm{H}\right) .{ }^{13} \mathrm{C}$ NMR $\left(100 \mathrm{MHz}, \mathrm{C}_{6} \mathrm{D}_{6}\right) \delta 140.64(P h), 128.52(P h), 127.31(P h), 127.19(P h), 88.53(\mathrm{C}-4), 83.89(\mathrm{C}-13), 83.23$ (C-10), 75.61 (C-1), 72.98 (C-14), 63.18 (Bn), 46.63 (C-9), 45.02 (C-3), 42.60 (C-7), 40.87 (C-6), 37.69 (C-8), 33.86 (C-5), 32.95 (C-15), 32.42 (C-2), 32.18 (C-11), 28.08 (C-12), 25.64 (C-19), 24.62 (C-20), 19.71 (C-18), 18.71 (C-16, C-17), 18.65 (C-16, C-17). IR (Diamond-ATR, neat) $\tilde{v}_{\text {max }}: 3404$ (br), 2935 (s), 2869 (m), $1712(\mathrm{w}), 1453(\mathrm{~s}), 1378(\mathrm{~s}), 1256(\mathrm{~m}), 1113(\mathrm{~m}), 1048(\mathrm{~m}), 1030(\mathrm{~m}) \mathrm{cm}^{-1}$. HRMS (ESI): calcd for $\left(\left[\mathrm{M}+\mathrm{CH}_{3} \mathrm{COO}\right], \mathrm{C}_{29} \mathrm{H}_{43} \mathrm{O}_{6}\right)^{-}: 487.30596$, found: 487.30674 . $[\alpha]_{D}^{20}=+18.8^{\circ}\left(\mathrm{c}=0.23, \mathrm{CH}_{2} \mathrm{Cl}_{2}\right.$ ). 


\subsection{Synthesis of epoxide 16}<smiles>CC(C)[C@]1(O)CC[C@]2(C)C[C@]3(C)[C@H](O)CC[C@@H]4O[C@]4(C)[C@]3(O)C[C@@]21C</smiles>

15

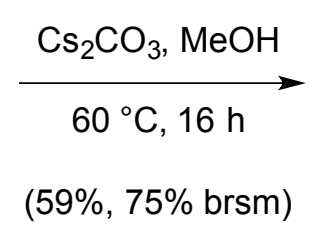

$(59 \%, 75 \%$ brsm $)$

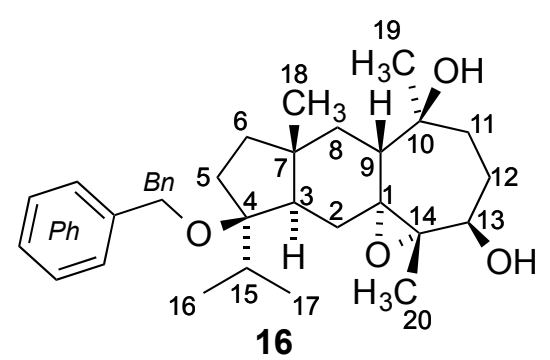

16

A solution of epoxide 15 (94.0 mg, $0.22 \mathrm{mmol}, 1$ equiv) in methanol (12 mL) was treated with cesium carbonate $\left(1.79 \mathrm{~g}, 5.48 \mathrm{mmol}, 25.0\right.$ equiv) and the resulting mixture was heated to $60^{\circ} \mathrm{C}$. After $16 \mathrm{~h}$, the mixture was allowed to cool to $23{ }^{\circ} \mathrm{C}$ and then was diluted with $\mathrm{pH} 7$ buffer solution $(30 \mathrm{~mL}$ ) and ethyl acetate $(25 \mathrm{~mL})$. The layers were separated, the aqueous layer was extracted with ethyl acetate $(3 \times 20 \mathrm{~mL})$ and the combined organic extracts were dried over sodium sulfate. The dried solution was filtered and the filtrate was concentrated. The residue was purified by flash-column chromatography on silica gel (50\% ethyl acetate in hexanes) to provide recovered epoxide 15 (21.0 $\mathrm{mg}, 22 \%)$ as a colourless oil and epoxide 16 (55.0 mg, 59\%, 75\% brsm) as a colourless oil.

TLC (50\% ethyl acetate in hexanes): $\mathrm{R}_{f}=0.15$ (CAM). ${ }^{1} \mathrm{H}$ NMR $\left(400 \mathrm{MHz}, \mathrm{C}_{6} \mathrm{D}_{6}\right) \delta 7.38-7.33(\mathrm{~m}, 2 \mathrm{H}, \mathrm{Ph})$, 7.27-7.21 (m, 2H, Ph), 7.15-7.10 (m, 1H, Ph), 4.27-4.18 (m, 2H, Bn), 3.92-3.88 (m, 1H, 13-H), 2.29 (dd, $\left.{ }^{2} J_{8 \mathrm{~A} / 8 \mathrm{~B}}=12.4 \mathrm{~Hz},{ }^{3} \mathrm{~J}_{8 \mathrm{~A} / 9}=4.1 \mathrm{~Hz}, 1 \mathrm{H}, 8-\mathrm{H}_{\mathrm{A}}\right), 2.23-2.13\left(\mathrm{~m}, 2 \mathrm{H}, 2-\mathrm{H}_{\mathrm{A}}, 9-\mathrm{H}\right), 2.08-1.95\left(\mathrm{~m}, 2 \mathrm{H}, 5-\mathrm{H}_{\mathrm{A}}, 15-\mathrm{H}\right)$, 1.86-1.77 (m, 3H, 2- $\left.\mathrm{H}_{B}, 3-\mathrm{H}, 11-\mathrm{H}_{\mathrm{A}}\right), 1.74-1.65\left(\mathrm{~m}, 1 \mathrm{H}, 12-\mathrm{H}_{\mathrm{A}}\right), 1.65-1.51\left(\mathrm{~m}, 3 \mathrm{H}, 5-\mathrm{H}_{B}, 6-\mathrm{H}_{\mathrm{A}}, 8-\mathrm{H}_{\mathrm{B}}\right), 1.47(\mathrm{~s}$, $3 \mathrm{H}, 20-\mathrm{H}), 1.44-1.35\left(\mathrm{~m}, 2 \mathrm{H}, 11-\mathrm{H}_{\mathrm{B}}, 12-\mathrm{H}_{\mathrm{B}}\right), 1.27(\mathrm{~s}, 3 \mathrm{H}, 19-\mathrm{H}), 1.23-1.14\left(\mathrm{~m}, 1 \mathrm{H}, 6-\mathrm{H}_{\mathrm{B}}\right), 1.12(\mathrm{~s}, 3 \mathrm{H}, 18-\mathrm{H})$, $0.98\left(\mathrm{~d},{ }^{3} \mathrm{~J}=6.8 \mathrm{~Hz}, 3 \mathrm{H}, 16-\mathrm{H}, 17-\mathrm{H}\right), 0.85\left(\mathrm{~d},{ }^{3} \mathrm{~J}=6.8 \mathrm{~Hz}, 3 \mathrm{H}, 16-\mathrm{H}, 17-\mathrm{H}\right) .{ }^{13} \mathrm{C}$ NMR $\left(100 \mathrm{MHz}, \mathrm{C}_{6} \mathrm{D}_{6}\right)$ $\delta 140.55(P h), 128.54(P h), 127.24(P h), 127.19(P h), 88.26$ (C-4), 76.33 (C-13), 74.70 (C-10), 67.17 (C-14), 66.39 (C-1), 63.03 (Bn), 48.63 (C-3), 46.83 (C-9), 41.83 (C-7, C-8), 41.55 (C-7, C-8), 41.19 (C-6), 36.95 (C-11), 34.40 (C-5), 33.57 (C-2), 33.09 (C-15), 27.67 (C-12), 25.53 (C-19), 23.19 (C-20), 18.85 (C-18), 18.33 (C-16, C-17), 17.99 (C-16, C-17). IR (Diamond-ATR, neat) $\tilde{v}_{\max }: 3383$ (br), 2954 (s), 2929 (s), $1451(\mathrm{~m}), 1382(\mathrm{~m}), 1346$ (w), 1091 (s), 1059 (s), 1030 (s) cm ${ }^{-1}$. HRMS (ESI): calcd for ([M+CH $\mathrm{COO}_{3}$, $\left.\mathrm{C}_{29} \mathrm{H}_{43} \mathrm{O}_{6}\right)^{-}: 487.30596$, found: 487.30682 . $[\alpha]_{D}^{20}=+35.0^{\circ}\left(\mathrm{c}=0.20, \mathrm{CH}_{2} \mathrm{Cl}_{2}\right.$ ). 


\subsection{Synthesis of triol 40}<smiles>CC(C)[C@]1(O)CC[C@]2(C)C[C@]3(C)[C@H](O)CC[C@@H](O)[C@]3(C)C[C@@]21C</smiles>

16

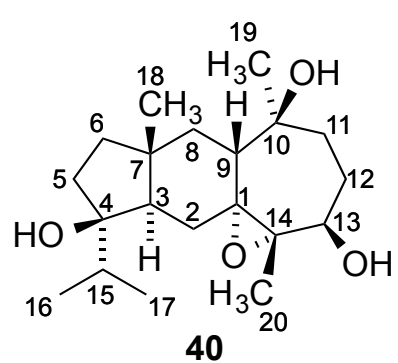

40

A solution of epoxide $16(7.5 \mathrm{mg}, 17.5 \mu \mathrm{mol}, 1$ equiv) in tetrahydrofuran $(2.5 \mathrm{~mL})$ was treated with palladium on carbon ( $10 \mathrm{wt} . \%, 46.6 \mathrm{mg}, 43.7 \mu \mathrm{mol}, 2.50$ equiv) at $23^{\circ} \mathrm{C}$. An atmosphere of hydrogen was maintained by sparging the mixture with a stream of hydrogen gas using a stainless steel needle for $5 \mathrm{~min}$ and vigorous stirring of the suspension was then continued under hydrogen atmosphere at $23^{\circ} \mathrm{C}$. After $1 \mathrm{~h}$, the mixture was diluted with ethyl acetate $(10 \mathrm{~mL})$ and filtered through a short pad of Celite. The filtrate was concentrated and the residue was purified by flash-column chromatography on silica gel (9\% methanol in dichloromethane) to provide triol $\mathbf{4 0}(4.7 \mathrm{mg}, 80 \%)$ as a colourless solid.

Recrystallization (ethyl acetate/hexanes) of the product gave crystals suitable for X-ray diffraction. $\operatorname{TLC}$ (9\% methanol in dichloromethane): $\mathrm{R}_{f}=0.15$ (CAM). ${ }^{1} \mathrm{H}$ NMR (800 MHz, $\left.\mathrm{CD}_{2} \mathrm{Cl}_{2}\right) \delta 4.21$ (dd, $\left.{ }^{3} J_{13 / 12 \mathrm{~B}}=4.8 \mathrm{~Hz},{ }^{3} J_{13 / 12 \mathrm{~A}}=2.5 \mathrm{~Hz}, 1 \mathrm{H}, 13-\mathrm{H}\right), 2.17-2.13\left(\mathrm{~m}, 2 \mathrm{H}, 8-\mathrm{H}_{\mathrm{A}}, 9-\mathrm{H}\right), 2.04-2.00\left(\mathrm{~m}, 1 \mathrm{H}, 5-\mathrm{H}_{\mathrm{A}}\right)$, 1.87-1.80 (m, 2H, 2- $\left.\mathrm{H}_{\mathrm{A}}, 11-\mathrm{H}_{\mathrm{A}}\right), 1.76-1.61\left(\mathrm{~m}, 5 \mathrm{H}, 5-\mathrm{H}_{\mathrm{B}}, 6-\mathrm{H}_{\mathrm{A}}, 12-\mathrm{H}, 15-\mathrm{H}\right), 1.53-1.48\left(\mathrm{~m}, 5 \mathrm{H}, 3-\mathrm{H}, 11-\mathrm{H}_{\mathrm{B}}\right.$, $20-\mathrm{H}), 1.46\left(\mathrm{dd},{ }^{2} \mathrm{~J}_{2 \mathrm{~B} / 2 \mathrm{~A}}=14.0 \mathrm{~Hz},{ }^{3} \mathrm{~J}_{2 \mathrm{~B} / 3}=3.2 \mathrm{~Hz}, 1 \mathrm{H}, 2-\mathrm{H}_{\mathrm{B}}\right), 1.41\left(\mathrm{app} \mathrm{t},{ }^{2} \mathrm{~J}_{8 \mathrm{~B} / 8 \mathrm{~A}}={ }^{3} \mathrm{~J}_{8 \mathrm{~B} / 9}=13.3 \mathrm{~Hz}, 1 \mathrm{H}, 8-\mathrm{H}_{\mathrm{B}}\right)$, $1.20(\mathrm{~s}, 3 \mathrm{H}, 19-\mathrm{H}), 1.18-1.11\left(\mathrm{~m}, 1 \mathrm{H}, 6-\mathrm{H}_{\mathrm{B}}\right), 1.05(\mathrm{~s}, 3 \mathrm{H}, 18-\mathrm{H}), 0.91(\mathrm{~d}, 3 \mathrm{~J}=6.8 \mathrm{~Hz}, 3 \mathrm{H}, 16-\mathrm{H}, 17-\mathrm{H})$, $0.88\left(\mathrm{~d},{ }^{3} \mathrm{~J}=6.8 \mathrm{~Hz}, 3 \mathrm{H}, 16-\mathrm{H}, 17-\mathrm{H}\right) .{ }^{13} \mathrm{C}$ NMR $\left(200 \mathrm{MHz}, \mathrm{CD}_{2} \mathrm{Cl}_{2}\right)$ \& 83.97 (C-4), 76.79 (C-13), 75.36 (C-10), 67.30 (C-14), 66.40 (C-1), 51.39 (C-3), 46.85 (C-9), 42.09 (C-8), 41.33 (C-7), 40.40 (C-6), 37.94 (C-15), 37.21 (C-11), 37.15 (C-5), 31.69 (C-2), 27.76 (C-12), 25.34 (C-19), 23.15 (C-20), 18.90 (C-18), 18.55 (C-16, C-17), 17.78 (C-16, C-17). IR (Diamond-ATR, neat) $\tilde{v}_{\max }: 3424$ (br), 2957 (s), 2924 (s), 2867 (m), 1455 (m), $1382(\mathrm{~m}), 1261(\mathrm{~m}), 1095(\mathrm{~s}), 1005(\mathrm{~s}) \mathrm{cm}^{-1}$. HRMS (ESI): calcd for $\left(\left[\mathrm{M}+\mathrm{CH}_{3} \mathrm{COO}\right], \mathrm{C}_{22} \mathrm{H}_{37} \mathrm{O}_{6}\right)^{-}: 397.25901$, found: $397.26046 .[\alpha]_{D}^{20}=+9.4^{\circ}(c=0.11, \mathrm{MeOH})$. 


\subsection{Synthesis of 1,10,13,14-tetra-epi dictyoxetane (tetra-epi-1)}

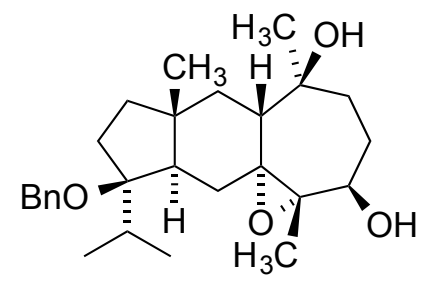

16

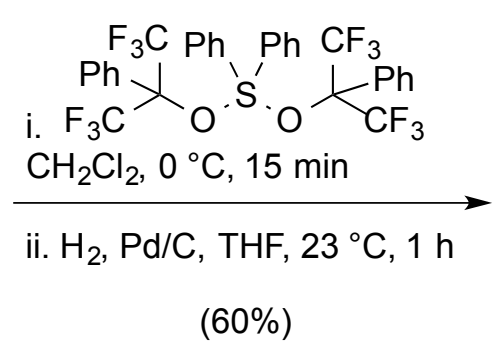

$(60 \%)$

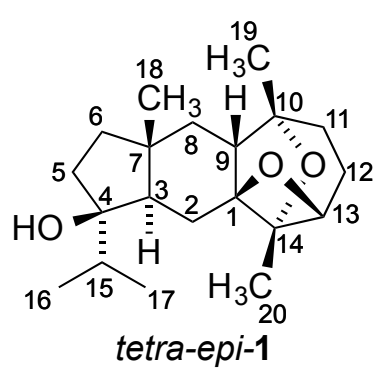

A solution of epoxide $16(5.0 \mathrm{mg}, 11.7 \mu \mathrm{mol}, 1$ equiv) in dichloromethane $(1.2 \mathrm{~mL})$ was treated with Martin sulfurane $\left(7.9 \mathrm{mg}, 11.7 \mu \mathrm{mol}, 1.00\right.$ equiv) at $0{ }^{\circ} \mathrm{C}$. After $15 \mathrm{~min}$, the mixture was diluted with $\mathrm{pH} 7$ buffer solution $(10 \mathrm{~mL})$ and diethyl ether $(10 \mathrm{~mL})$. The layers were separated, the aqueous layer was extracted with diethyl ether $(3 \times 10 \mathrm{~mL})$ and the combined organic extracts were dried over sodium sulfate. The dried solution was filtered and the filtrate was concentrated. The residue was filtered through a pad of silica gel (10\% ethyl acetate in hexanes) to provide dioxatricycle $17^{9}$ which was used in the next step without further purification.

A solution of the crude dioxatricycle 17 (assuming $11.7 \mu \mathrm{mol}$ ) in tetrahydrofuran $(2.5 \mathrm{~mL}$ ) was treated with palladium on carbon (10 wt.\%, $24.9 \mathrm{mg}, 23.4 \mu \mathrm{mol}, 2.00$ equiv) at $23{ }^{\circ} \mathrm{C}$. An atmosphere of hydrogen was maintained by sparging the mixture with a stream of hydrogen gas using a stainless steel needle for $5 \mathrm{~min}$ and vigorous stirring of the suspension was then continued under hydrogen atmosphere at $23^{\circ} \mathrm{C}$. After $1 \mathrm{~h}$, the mixture was diluted with ethyl acetate $(10 \mathrm{~mL})$ and filtered through a short pad of Celite. The filtrate was concentrated and the residue was purified by flash-column chromatography on silica gel (35\% ethyl acetate in hexanes) to provide 1,10,13,14-tetra-epi dictyoxetane (tetra-epi-1) (2.3 mg, 60\% over two steps) as a colourless solid.

Recrystallization (ethyl acetate/hexanes) of the product gave crystals suitable for X-ray diffraction.

$\operatorname{TLC}$ (40\% ethyl acetate in hexanes): $\mathrm{R}_{f}=0.26$ (CAM). ${ }^{1} \mathrm{H} \mathbf{N M R}\left(800 \mathrm{MHz}, \mathrm{C}_{6} \mathrm{D}_{6}\right) \delta 4.29$ (br d, $\left.{ }^{3} J_{13 / 12 \mathrm{~A}}=3.6 \mathrm{~Hz}, 1 \mathrm{H}, 13-\mathrm{H}\right), 2.08-2.02\left(\mathrm{~m}, 2 \mathrm{H}, 2-\mathrm{H}_{\mathrm{A}}, 9-\mathrm{H}\right), 2.02-1.96\left(\mathrm{~m}, 2 \mathrm{H}, 2-\mathrm{H}_{\mathrm{B}}, 11-\mathrm{H}_{\mathrm{A}}\right), 1.86-1.78(\mathrm{~m}$, $\left.2 \mathrm{H}, 5-\mathrm{H}_{\mathrm{A}}, 12-\mathrm{H}_{\mathrm{A}}\right), 1.74-1.61\left(\mathrm{~m}, 3 \mathrm{H}, 5-\mathrm{H}_{\mathrm{B}}, 11-\mathrm{H}_{\mathrm{B}}, 12-\mathrm{H}_{\mathrm{B}}\right), 1.56\left(\mathrm{dd},{ }^{2} \mathrm{~J}_{8 \mathrm{~A} / 8 \mathrm{~B}}=12.6 \mathrm{~Hz},{ }^{3} \mathrm{~J}_{8 \mathrm{~A} / 9}=6.5 \mathrm{~Hz}, 1 \mathrm{H}\right.$, 8- $\left.\mathrm{H}_{\mathrm{A}}\right), 1.49-1.43\left(\mathrm{~m}, 2 \mathrm{H}, 6-\mathrm{H}_{\mathrm{A}}, 15-\mathrm{H}\right), 1.30(\mathrm{~s}, 3 \mathrm{H}, 20-\mathrm{H}), 1.19(\mathrm{~s}, 3 \mathrm{H}, 19-\mathrm{H}), 1.09(\mathrm{~s}, 3 \mathrm{H}, 18-\mathrm{H}), 1.02$ (dd, $\left.{ }^{3} J_{3 / 2 \mathrm{~B}}=14.0 \mathrm{~Hz},{ }^{3} \mathrm{~J}_{3 / 2 \mathrm{~A}}=3.6 \mathrm{~Hz}, 1 \mathrm{H}, 3-\mathrm{H}\right), 0.92-0.85\left(\mathrm{~m}, 5 \mathrm{H}, 6-\mathrm{H}_{\mathrm{B}}, 8-\mathrm{H}_{\mathrm{B}}, 16-\mathrm{H}, 17-\mathrm{H}\right), 0.78\left(\mathrm{~d},{ }^{3} \mathrm{~J}=6.8 \mathrm{~Hz}, 3 \mathrm{H}\right.$, 16-H, 17-H), 0.69 (br s, 1H, 4-OH). ${ }^{13} \mathrm{C}$ NMR (100 MHz, C $\left.6 \mathrm{D}_{6}\right) \delta 96.98$ (C-1), 82.90 (C-4), 82.37 (C-14), 81.52 (C-13), 81.15 (C-10), 51.95 (C-9), 50.37 (C-3), 41.47 (C-7), 41.15 (C-8), 39.80 (C-6), 37.65 (C-15), 36.83 (C-5), 33.06 (C-11), 28.72 (C-2), 24.18 (C-12), 24.11 (C-19), 18.53 (C-18), 18.49 (C-16, C-17), 17.80 (C-16, C-17), 17.29 (C-20). IR (Diamond-ATR, neat) $\tilde{v}_{\text {max }}: 3455(\mathrm{~m}), 2958(\mathrm{~s}), 2932$ (s), 2852 (m), $1454(\mathrm{~m}), 1384(\mathrm{~m}), 1275(\mathrm{~m}), 1189(\mathrm{~m}), 1121(\mathrm{~m}), 1062(\mathrm{~m}), 1020(\mathrm{~m}), 974(\mathrm{~m}) \mathrm{cm}^{-1}$. HRMS (El): calcd for $\left([\mathrm{M}], \mathrm{C}_{20} \mathrm{H}_{32} \mathrm{O}_{3}\right)^{+}: 320.2351$, found: $320.2351 .[\alpha]_{D}^{20}=-90.0^{\circ}\left(\mathrm{c}=0.07, \mathrm{CH}_{2} \mathrm{Cl}_{2}\right.$ ). 


\subsection{Synthesis of bromohydrin 20}

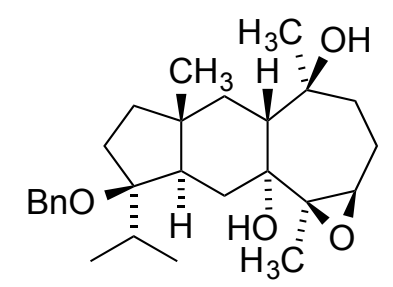

15

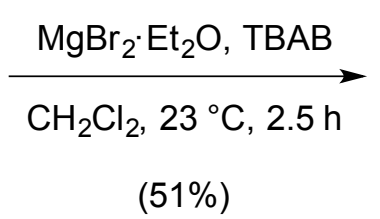

$(51 \%)$

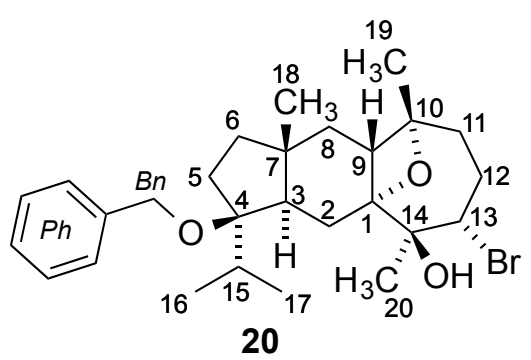

20

A solution of epoxide $15(25.0 \mathrm{mg}, 58.3 \mu \mathrm{mol}, 1$ equiv) in dichloromethane $(3 \mathrm{~mL})$ was treated sequentially with magnesium bromide ethyl etherate $(90.4 \mathrm{mg}, 350 \mu \mathrm{mol}, 6.00$ equiv) and tetrabutylammonium bromide ( $113 \mathrm{mg}, 350 \mu \mathrm{mol}, 6.00$ equiv) at $0{ }^{\circ} \mathrm{C}$. After $10 \mathrm{~min}$, the mixture was warmed to $23{ }^{\circ} \mathrm{C} .{ }^{10}$ After $2.5 \mathrm{~h}$, the mixture was diluted with $\mathrm{pH} 7$ buffer solution $(15 \mathrm{~mL})$ and diethyl ether $(10 \mathrm{~mL})$. The layers were separated, the aqueous layer was extracted with diethyl ether $(3 \times 10 \mathrm{~mL})$ and the combined organic extracts were dried over sodium sulfate. The dried solution was filtered and the filtrate was concentrated. The residue was purified by flash-column chromatography on silica gel (8\% ethyl acetate in hexanes) to provide bromohydrine $20(14.5 \mathrm{mg}, 51 \%)$ as a colourless oil.

TLC (10\% ethyl acetate in hexanes): $\mathrm{R}_{f}=0.39$ (CAM). ${ }^{1} \mathrm{H}$ NMR $\left(800 \mathrm{MHz}, \mathrm{C}_{6} \mathrm{D}_{6}\right) \delta 7.45-7.42(\mathrm{~m}, 2 \mathrm{H}, P h)$, 7.27-7.23 (m, 2H, Ph), 7.11-7.08 (m, 1H,Ph), $4.68\left(\mathrm{~d},{ }^{3} \mathrm{~J}_{13 / 12 \mathrm{~A}}=13.2 \mathrm{~Hz}, 1 \mathrm{H}, 13-\mathrm{H}\right), 4.37-4.27(\mathrm{~m}, 2 \mathrm{H}, \mathrm{Bn})$, $2.65\left(\right.$ app t, $\left.{ }^{2} J_{2 \mathrm{~A} / 2 \mathrm{~B}}={ }^{3} J_{2 \mathrm{~A} / 3}=13.9 \mathrm{~Hz}, 1 \mathrm{H}, 2-\mathrm{H}_{\mathrm{A}}\right), 2.42-2.35\left(\mathrm{~m}, 1 \mathrm{H}, 12-\mathrm{H}_{\mathrm{A}}\right), 2.33\left(\mathrm{dd},{ }^{3} \mathrm{~J}_{9 / 8 \mathrm{~A}}=10.5 \mathrm{~Hz}\right.$, $\left.{ }^{3} J_{9 / 8 \mathrm{~B}}=6.6 \mathrm{~Hz}, 1 \mathrm{H}, 9-\mathrm{H}\right), 2.11-2.04\left(\mathrm{~m}, 3 \mathrm{H}, 5-\mathrm{H}_{\mathrm{A}}, 12-\mathrm{H}_{\mathrm{B}}, 15-\mathrm{H}\right), 2.00\left(\mathrm{dd},{ }^{3} 3_{3 / 2 \mathrm{~A}}=13.9 \mathrm{~Hz},{ }^{3} J_{3 / 2 \mathrm{~B}}=4.1 \mathrm{~Hz}, 1 \mathrm{H}\right.$, $3-\mathrm{H}), 1.76-1.69\left(\mathrm{~m}, 3 \mathrm{H}, 2-\mathrm{H}_{\mathrm{B}}, 5-\mathrm{H}_{\mathrm{B}}, 14-\mathrm{OH}\right), 1.65\left(\mathrm{dd},{ }^{2} \mathrm{~J}_{6 \mathrm{~A} / 6 \mathrm{~B}}=11.9 \mathrm{~Hz},{ }^{3} J_{6 \mathrm{~A} / 5 \mathrm{~A}}=7.7 \mathrm{~Hz}, 1 \mathrm{H}, 6-\mathrm{H}_{\mathrm{A}}\right), 1.63-1.58$ $(\mathrm{m}, 2 \mathrm{H}, 8-\mathrm{H}), 1.36(\mathrm{~s}, 3 \mathrm{H}, 20-\mathrm{H}), 1.35-1.28\left(\mathrm{~m}, 1 \mathrm{H}, 11-\mathrm{H}_{\mathrm{A}}\right), 1.24-1.19\left(\mathrm{~m}, 4 \mathrm{H}, 6-\mathrm{H}_{\mathrm{B}}, 18-\mathrm{H}\right), 1.13-1.07(\mathrm{~m}$, $\left.1 \mathrm{H}, 11-\mathrm{H}_{\mathrm{B}}\right), 1.06(\mathrm{~s}, 3 \mathrm{H}, 19-\mathrm{H}), 1.03\left(\mathrm{~d},{ }^{3} \mathrm{~J}=6.8 \mathrm{~Hz}, 3 \mathrm{H}, 16-\mathrm{H}, 17-\mathrm{H}\right), 0.95\left(\mathrm{~d},{ }^{3} \mathrm{~J}=6.8 \mathrm{~Hz}, 3 \mathrm{H}, 16-\mathrm{H}, 17-\mathrm{H}\right)$. ${ }^{13} \mathrm{C}$ NMR (100 MHz, $\left.\mathrm{C}_{6} \mathrm{D}_{6}\right) \delta 140.73(P h), 128.58(P h), 127.19(P h), 127.08(P h), 88.25(\mathrm{C}-4), 84.37(\mathrm{C}-1)$, 81.33 (C-10), 76.97 (C-14), 69.48 (C-13), 63.38 (Bn), 44.71 (C-3), 41.71 (C-6), 40.53 (C-7), 40.49 (C-11), 38.90 (C-9), 37.35 (C-8), 35.61 (C-5), 33.77 (C-15), 32.83 (C-12), 30.56 (C-2), 25.65 (C-19), 20.21 (C-18), 18.40 (C-16, C-17), 17.76 (C-16, C-17), 17.48 (br, C-20). IR (Diamond-ATR, neat) $\tilde{v}_{\text {max }} 3432$ (br), 2952 (s), $2865(\mathrm{~m}), 1453(\mathrm{~m}), 1375(\mathrm{~s}), 1197(\mathrm{w}), 1129(\mathrm{~m}), 1098(\mathrm{~m}), 1060(\mathrm{~m}), 1027(\mathrm{w}) \mathrm{cm}^{-1}$. HRMS (ESI): calcd for ([M+CH $\left.\left.\mathrm{CH}_{3} \mathrm{COO}\right], \mathrm{C}_{29} \mathrm{H}_{42}{ }^{79} \mathrm{BrO}_{5}\right)^{-}:$549.22156, found: 549.22240 ; calcd for ([M+C $\left.\left.\mathrm{CH}_{3} \mathrm{COO}\right], \mathrm{C}_{29} \mathrm{H}_{42}{ }^{81} \mathrm{BrO}_{5}\right)^{-}$: 551.21952, found: 551.22035. $[\alpha]_{D}^{20}=-28.5^{\circ}\left(\mathrm{c}=0.71, \mathrm{CH}_{2} \mathrm{Cl}_{2}\right.$ ). 


\subsection{Synthesis of diol 21}

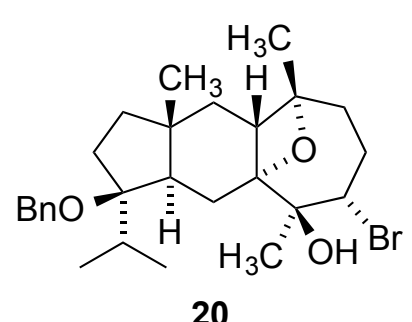

20

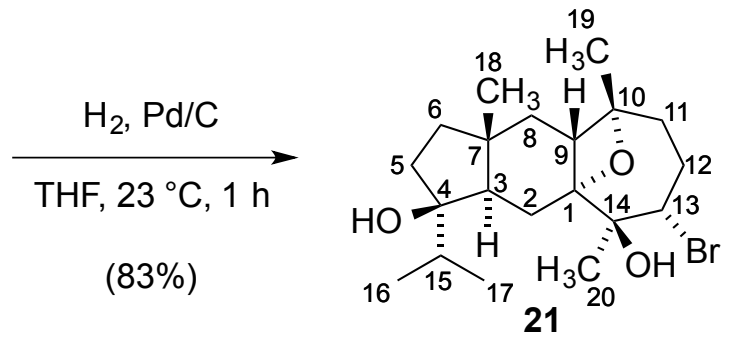

$(83 \%)$

A solution of bromohydrin 20 (4.0 mg, $8.14 \mu \mathrm{mol}, 1$ equiv) in tetrahydrofuran $(2.5 \mathrm{~mL})$ was treated with palladium on carbon ( $10 \mathrm{wt} . \%, 8.7 \mathrm{mg}, 8.14 \mu \mathrm{mol}, 1.00$ equiv) at $23^{\circ} \mathrm{C}$. An atmosphere of hydrogen was maintained by sparging the mixture with a stream of hydrogen gas using a stainless steel needle for $5 \mathrm{~min}$ and vigorous stirring of the suspension was then continued under hydrogen atmosphere at $23^{\circ} \mathrm{C}$. After $1 \mathrm{~h}$, the mixture was diluted with ethyl acetate $(10 \mathrm{~mL})$ and filtered through a short pad of Celite. The filtrate was concentrated and the residue was purified by flash-column chromatography on silica gel (20\% ethyl acetate in hexanes) to provide diol 21 (2.7 $\mathrm{mg}, \geq 99 \%)$ as a colourless solid.

Recrystallization (ethyl acetate/hexanes) of the product gave crystals suitable for X-ray diffraction.

$\operatorname{TLC}$ (30\% ethyl acetate in hexanes): $\mathrm{R}_{f}=0.35$ (CAM). ${ }^{1} \mathbf{H} \mathbf{N M R}\left(400 \mathrm{MHz}, \mathrm{C}_{6} \mathrm{D}_{6}\right) \delta 4.68$ (dd, $\left.{ }^{3} J_{13 / 12 \mathrm{~A}}=13.2 \mathrm{~Hz},{ }^{3} J_{13 / 12 \mathrm{~B}}=2.0 \mathrm{~Hz}, 1 \mathrm{H}, 13-\mathrm{H}\right), 2.47-2.33\left(\mathrm{~m}, 1 \mathrm{H}, 12-\mathrm{H}_{\mathrm{A}}\right), 2.33-2.23\left(\mathrm{~m}, 2 \mathrm{H}, 2-\mathrm{H}_{\mathrm{A}}, 9-\mathrm{H}\right)$, 2.11-2.04 (m, $\left.1 \mathrm{H}, 12-\mathrm{H}_{B}\right), 1.99-1.88\left(\mathrm{~m}, 2 \mathrm{H}, 5-\mathrm{H}_{\mathrm{A}}, 14-\mathrm{OH}\right), 1.79-1.64\left(\mathrm{~m}, 3 \mathrm{H}, 3-\mathrm{H}, 5-\mathrm{H}_{B}, 6-\mathrm{H}_{\mathrm{A}}\right)$, 1.57-1.42 (m, 7H, 2- $\left.\mathrm{H}_{\mathrm{B}}, 8-\mathrm{H}, 15-\mathrm{H}, 20-\mathrm{H}\right), 1.36-1.27\left(\mathrm{~m}, 1 \mathrm{H}, 11-\mathrm{H}_{\mathrm{A}}\right), 1.21(\mathrm{~s}, 3 \mathrm{H}, 18-\mathrm{H}), 1.17-1.03(\mathrm{~m}, 2 \mathrm{H}$, $\left.6-\mathrm{H}_{\mathrm{B}}, 11-\mathrm{H}_{\mathrm{B}}\right), 1.00(\mathrm{~s}, 3 \mathrm{H}, 19-\mathrm{H}), 0.91\left(\mathrm{~d},{ }^{3} \mathrm{~J}=6.8 \mathrm{~Hz}, 3 \mathrm{H}, 16-\mathrm{H}, 17-\mathrm{H}\right), 0.86\left(\mathrm{~d},{ }^{3} \mathrm{~J}=6.8 \mathrm{~Hz}, 3 \mathrm{H}, 16-\mathrm{H}, 17-\mathrm{H}\right)$, 0.71 (br s, $1 \mathrm{H}, 4-\mathrm{OH}) .{ }^{13} \mathrm{C}$ NMR (100 MHz, $\left.\mathrm{C}_{6} \mathrm{D}_{6}\right) \delta 84.05$ (C-1), 83.49 (C-4), 81.48 (C-10), 77.14 (C-14), 69.31 (C-13), 47.64 (C-3), 40.92 (C-6), 40.45 (C-11), 40.24 (C-7), 38.89 (C-9), 37.97 (C-8, C-15), 37.92 (C-8, C-15), 37.04 (C-5), 32.84 (C-12), 28.48 (C-2), 25.58 (C-19), 20.19 (C-18), 18.36 (C-16, C-17), 17.66 (2C, C-16, C-17, C-20). IR (Diamond-ATR, neat) $\tilde{v}_{\max }: 3460(\mathrm{br}), 2954(\mathrm{~s}), 2869(\mathrm{~m}), 1468(\mathrm{~m}), 1447(\mathrm{~m})$, $1374(\mathrm{~m}), 1129(\mathrm{~m}), 1094(\mathrm{~m}), 1074(\mathrm{~m}), 986(\mathrm{~m}) \mathrm{cm}^{-1}$. HRMS (ESI): calcd for $\left(\left[\mathrm{M}+\mathrm{CH}_{3} \mathrm{COO}\right]\right.$, $\left.\mathrm{C}_{22} \mathrm{H}_{36}{ }^{79} \mathrm{BrO}_{5}\right)^{-}:$:459.17461, found: 459.17549; calcd for ([M+CH $\left.\left.\mathrm{CH}_{3} \mathrm{COO}\right], \mathrm{C}_{22} \mathrm{H}_{36}{ }^{81} \mathrm{BrO}_{5}\right)^{-}: 461.17257$, found: 461.17345. $[\alpha]_{D}^{20}=-52.5^{\circ}\left(\mathrm{c}=0.35, \mathrm{CH}_{2} \mathrm{Cl}_{2}\right)$. 


\subsection{Synthesis of epoxide-oxetane 22}

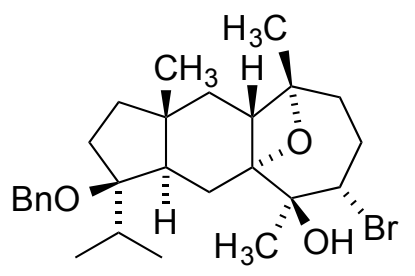

20

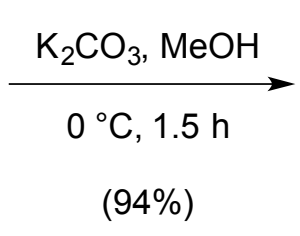

$(94 \%)$

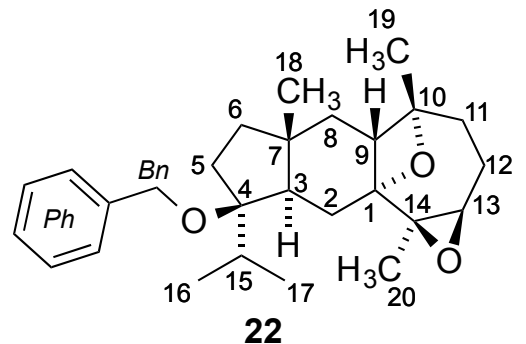

22

A solution of bromohydrin 20 (14.0 mg, $28.5 \mu \mathrm{mol}, 1$ equiv) in methanol $(1.5 \mathrm{~mL})$ was treated with potassium carbonate $\left(9.8 \mathrm{mg}, 71.2 \mu \mathrm{mol}, 2.50\right.$ equiv) at $0{ }^{\circ} \mathrm{C}$. After $1.5 \mathrm{~h}$, the mixture was diluted with $\mathrm{pH} 7$ buffer solution $(15 \mathrm{~mL})$ and diethyl ether $(10 \mathrm{~mL})$. The layers were separated, the aqueous layer was extracted with diethyl ether $(3 \times 10 \mathrm{~mL})$ and the combined organic extracts were dried over sodium sulfate. The dried solution was filtered and the filtrate was concentrated. The residue was purified by flash-column chromatography on silica gel (20\% ethyl acetate in hexanes) to provide epoxide-oxetane $\mathbf{2 2}$ (11.0 $\mathrm{mg}, 94 \%)$ as a colourless oil.

TLC (20\% ethyl acetate in hexanes): $\mathrm{R}_{f}=0.28$ (CAM). ${ }^{1} \mathrm{H}$ NMR $\left(400 \mathrm{MHz}, \mathrm{C}_{6} \mathrm{D}_{6}\right) \delta 7.41-7.35(\mathrm{~m}, 2 \mathrm{H}, \mathrm{Ph})$, 7.22-7.17 (m, 2H, Ph), 7.12-7.06 (m, 1H, Ph), 4.34-4.21 (m, 2H, Bn), 2.86-2.77 (m, 1H, 9-H), 2.73 (dd, $\left.{ }^{3} J_{13 / 12 \mathrm{~B}}=7.8 \mathrm{~Hz},{ }^{3} J_{13 / 12 \mathrm{~A}}=3.1 \mathrm{~Hz}, 1 \mathrm{H}, 13-\mathrm{H}\right), 2.45\left(\mathrm{dd},{ }^{2} J_{2 \mathrm{~A} / 2 \mathrm{~B}}=14.8 \mathrm{~Hz},{ }^{3} J_{2 \mathrm{~A} / 3}=13.0 \mathrm{~Hz}, 1 \mathrm{H}, 2-\mathrm{H}_{\mathrm{A}}\right)$, 2.08-1.95 (m, 4H, 3-H, 5- $\left.\mathrm{H}_{\mathrm{A}}, 12-\mathrm{H}_{\mathrm{A}}, 15-\mathrm{H}\right), 1.93-1.80\left(\mathrm{~m}, 3 \mathrm{H}, 2-\mathrm{H}_{B}, 11-\mathrm{H}_{\mathrm{A}}, 12-\mathrm{H}_{\mathrm{B}}\right), 1.72-1.62\left(\mathrm{~m}, 3 \mathrm{H}, 5-\mathrm{H}_{B}\right.$, 8-H), 1.62-1.54 (m, 1H, 6- $\left.\mathrm{H}_{\mathrm{A}}\right), 1.29(\mathrm{~s}, 3 \mathrm{H}, 20-\mathrm{H}), 1.28-1.17\left(\mathrm{~m}, 2 \mathrm{H}, 6-\mathrm{H}_{\mathrm{B}}, 11-\mathrm{H}_{\mathrm{B}}\right), 1.11(\mathrm{~s}, 3 \mathrm{H}, 19-\mathrm{H})$, $1.10(\mathrm{~s}, 3 \mathrm{H}, 18-\mathrm{H}), 1.02\left(\mathrm{~d},{ }^{3} \mathrm{~J}=6.8 \mathrm{~Hz}, 3 \mathrm{H}, 16-\mathrm{H}, 17-\mathrm{H}\right), 0.93\left(\mathrm{~d},{ }^{3} \mathrm{~J}=6.8 \mathrm{~Hz}, 3 \mathrm{H}, 16-\mathrm{H}, 17-\mathrm{H}\right) .{ }^{13} \mathrm{C}$ NMR $\left(100 \mathrm{MHz}, \mathrm{C}_{6} \mathrm{D}_{6}\right) \delta 140.61(P h), 128.61(P h), 127.14(P h), 126.90(P h), 88.18(\mathrm{C}-4), 83.25(\mathrm{C}-10), 82.82$ (C-1), 66.30 (C-14), 63.25 (Bn), 61.39 (C-13), 45.27 (C-3), 41.07 (C-6), 40.69 (C-7), 38.70 (C-11), 37.36 (C-8), 35.44 (C-5), 35.20 (C-9), 33.63 (C-15), 32.64 (C-2), 26.31 (C-19), 25.30 (C-12), 19.67 (C-18), 19.15 (C-20), 18.29 (C-16, C-17), 17.67 (C-16, C-17). IR (Diamond-ATR, neat) $\tilde{v}_{\max }: 2940$ (s), 2873 (m), $1496(\mathrm{~m}), 1453$ (s), 1376 (s), $1298(\mathrm{w}), 1149(\mathrm{~m}), 1089(\mathrm{~m}), 1055$ (s), $1028(\mathrm{~m}) \mathrm{cm}^{-1}$. HRMS (El): calcd for ([M], $\left.\mathrm{C}_{27} \mathrm{H}_{38} \mathrm{O}_{3}\right)^{+}:$410.2821, found: 410.2822. $[\alpha]_{D}^{20}=-9.3^{\circ}\left(\mathrm{c}=0.67, \mathrm{CH}_{2} \mathrm{Cl}_{2}\right.$ ). 


\subsection{Synthesis of dioxatricycle 17}

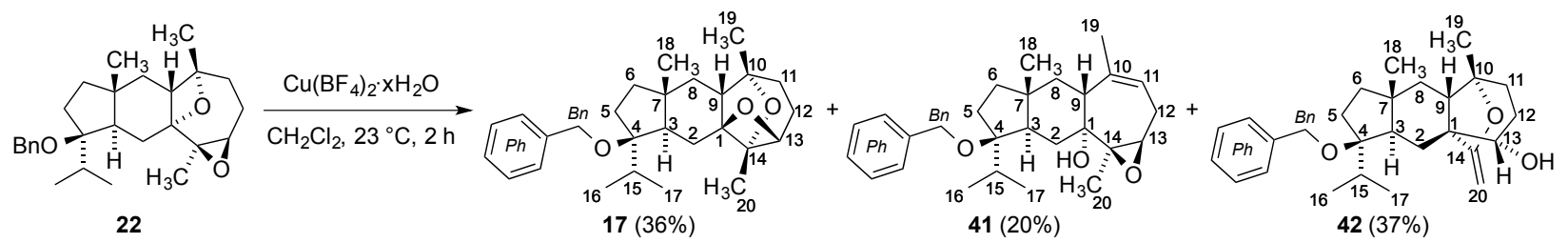

A solution of epoxide-oxetane $22(7.0 \mathrm{mg}, 17.0 \mu \mathrm{mol}, 1$ equiv) in dichloromethane $(1 \mathrm{~mL})$ was treated with copper(II) tetrafluoroborate hydrate $\left(4.4 \mathrm{mg}, 17.0 \mu \mathrm{mol}, 1.00\right.$ equiv) at $23^{\circ} \mathrm{C}$. After $2 \mathrm{~h}$, the yellow mixture was diluted with $\mathrm{pH} 7$ buffer solution $(15 \mathrm{~mL})$ and diethyl ether $(10 \mathrm{~mL})$. The layers were separated, the aqueous layer was extracted with diethyl ether $(3 \times 10 \mathrm{~mL})$ and the combined organic extracts were dried over sodium sulfate. The dried solution was filtered and the filtrate was concentrated. The residue was purified by flash-column chromatography on silica gel $(10 \% \rightarrow 20 \%$ ethyl acetate in hexanes) to provide dioxatricycle $17(2.5 \mathrm{mg}, 36 \%)$ as a colourless solid, olefin 41 (1.4 mg, $20 \%)$ as a colourless oil, and enol ether $\mathbf{4 2}(2.6 \mathrm{mg}, 37 \%)$ as a yellowish oil.

Dioxatricycle 17: TLC (20\% ethyl acetate in hexanes): $\mathrm{R}_{f}=0.45$ (CAM). ${ }^{1} \mathbf{H}$ NMR (400 MHz, $\mathrm{C}_{6} \mathrm{D}_{6}$ ) ס 7.40-7.34 (m, 2H, Ph), 7.24-7.18 (m, 2H, Ph), 7.14-7.08 (m, 1H, Ph), 4.31-4.17 (m, 3H, 13-H, Bn), 2.49-2.38 (m, 2H, 2-H), 2.10-1.91 (m, 4H, 5- $\left.\mathrm{H}_{\mathrm{A}}, 9-\mathrm{H}, 11-\mathrm{H}_{\mathrm{A}}, 15-\mathrm{H}\right), 1.87-1.79\left(\mathrm{~m}, 1 \mathrm{H}, 12-\mathrm{H}_{\mathrm{A}}\right), 1.76-1.62$ $\left(\mathrm{m}, 2 \mathrm{H}, 11-\mathrm{H}_{B}, 12-\mathrm{H}_{B}\right), 1.62-1.50\left(\mathrm{~m}, 2 \mathrm{H}, 5-\mathrm{H}_{B}, 8-\mathrm{H}_{\mathrm{A}}\right), 1.48-1.39\left(\mathrm{~m}, 1 \mathrm{H}, 6-\mathrm{H}_{\mathrm{A}}\right), 1.38(\mathrm{~s}, 3 \mathrm{H}, 20-\mathrm{H})$, 1.24-1.17 (m, 4H, 3-H, 19-H), $1.05(\mathrm{~s}, 3 \mathrm{H}, 18-\mathrm{H}), 1.02-0.92\left(\mathrm{~m}, 5 \mathrm{H}, 6-\mathrm{H}_{\mathrm{B}}, 8-\mathrm{H}_{\mathrm{B}}, 16-\mathrm{H}, 17-\mathrm{H}\right), 0.77(\mathrm{~d}$, $\left.{ }^{3} \mathrm{~J}=6.7 \mathrm{~Hz}, 3 \mathrm{H}, 16-\mathrm{H}, 17-\mathrm{H}\right) .{ }^{13} \mathrm{C}$ NMR $\left(100 \mathrm{MHz}, \mathrm{C}_{6} \mathrm{D}_{6}\right) \delta 140.43(P h), 128.58(P h), 127.15(P h)$, $126.96(P h), 97.31$ (C-1), 87.70 (C-4), 82.52 (C-14), 81.38 (C-13), 81.21 (C-10), 62.76 (Bn), 52.00 (C-9), 47.78 (C-3), 41.83 (C-7), 40.73 (C-6, C-8), 40.63 (C-6, C-8), 34.86 (C-5), 33.17 (C-11, C-15), 33.14 (C-11, C-15), 30.89 (C-2), 24.19 (C-19), 24.12 (C-12), 18.47 (C-16, C-17), 18.38 (C-18), 18.08 (C-16, C-17), 17.71 (C-20). IR (Diamond-ATR, neat) $\tilde{v}_{\max }: 2957$ (s), 2930 (s), $1454(\mathrm{~m}), 1385(\mathrm{~m}), 1273(\mathrm{w}), 1189(\mathrm{~m})$, $1089(\mathrm{~m}), 1057(\mathrm{~m}), 1027(\mathrm{~m}) \mathrm{cm}^{-1}$. HRMS (EI): calcd for ([M], $\left.\mathrm{C}_{27} \mathrm{H}_{38} \mathrm{O}_{3}\right)^{+}:$410.2821, found: 410.2816. $[\alpha]_{D}^{20}=-4.4^{\circ}\left(\mathrm{c}=0.36, \mathrm{CH}_{2} \mathrm{Cl}_{2}\right)$.

Olefin 41: TLC (20\% ethyl acetate in hexanes): $\mathrm{R}_{f}=0.38$ (CAM). ${ }^{1} \mathrm{H}$ NMR (400 MHz, $\left.\mathrm{C}_{6} \mathrm{D}_{6}\right) \delta 7.43-7.35$ (m, $2 \mathrm{H}, P h), 7.15-7.11(\mathrm{~m}, 2 \mathrm{H}, \mathrm{Ph}), 7.09-7.03(\mathrm{~m}, 1 \mathrm{H}, \mathrm{Ph}), 5.45-5.38(\mathrm{~m}, 1 \mathrm{H}, 11-\mathrm{H}), 4.37-4.24(\mathrm{~m}, 2 \mathrm{H}, B n)$, 2.85-2.76 (m, $2 \mathrm{H}, 9-\mathrm{H}, 13-\mathrm{H}), 2.50-2.36\left(\mathrm{~m}, 2 \mathrm{H}, 2-\mathrm{H}_{\mathrm{A}}, 12-\mathrm{H}_{\mathrm{A}}\right), 2.33\left(\mathrm{dd},{ }^{2} J_{2 \mathrm{~B} / 2 \mathrm{~A}}=14.1 \mathrm{~Hz},{ }^{3} J_{2 \mathrm{~B} / 3}=3.2 \mathrm{~Hz}, 1 \mathrm{H}\right.$, $\left.2-\mathrm{H}_{\mathrm{B}}\right), 2.16-1.97\left(\mathrm{~m}, 4 \mathrm{H}, 3-\mathrm{H}, 5-\mathrm{H}_{\mathrm{A}}, 12-\mathrm{H}_{\mathrm{B}}, 15-\mathrm{H}\right), 1.77\left(\mathrm{app} \mathrm{t},{ }^{2} \mathrm{~J}_{8 \mathrm{~A} / 8 \mathrm{~B}}={ }^{3} J_{8 \mathrm{~A} / 9}=12.5 \mathrm{~Hz}, 1 \mathrm{H}, 8-\mathrm{H}_{\mathrm{A}}\right), 1.69-1.60$ $\left(\mathrm{m}, 4 \mathrm{H}, 5-\mathrm{H}_{\mathrm{B}}, 19-\mathrm{H}\right), 1.60-1.53\left(\mathrm{~m}, 1 \mathrm{H}, 6-\mathrm{H}_{\mathrm{A}}\right), 1.49\left(\mathrm{dd},{ }^{2} \mathrm{~J}_{8 \mathrm{~B} / 8 \mathrm{~A}}=12.5 \mathrm{~Hz},{ }^{3} \mathrm{~J}_{8 \mathrm{~B} / 9}=4.9 \mathrm{~Hz}, 1 \mathrm{H}, 8-\mathrm{H}_{\mathrm{B}}\right), 1.22(\mathrm{~s}$, $3 \mathrm{H}, 20-\mathrm{H}), 1.22-1.17\left(\mathrm{~m}, 4 \mathrm{H}, 6-\mathrm{H}_{\mathrm{B}}, 18-\mathrm{H}\right), 1.08\left(\mathrm{~d},{ }^{3} \mathrm{~J}=6.8 \mathrm{~Hz}, 3 \mathrm{H}, 16-\mathrm{H}, 17-\mathrm{H}\right), 0.95\left(\mathrm{~d},{ }^{3} \mathrm{~J}=6.8 \mathrm{~Hz}, 3 \mathrm{H}\right.$, 16-H, 17-H). ${ }^{13} \mathrm{C}$ NMR (200 MHz, $\left.\mathrm{C}_{6} \mathrm{D}_{6}\right) \delta 140.53(\mathrm{C}-10, P h), 140.48(\mathrm{C}-10, P h), 128.54(P h), 127.04(P h)$, 127.00 (Ph), 124.86 (C-11), 88.59 (C-4), 75.15 (C-1), 65.40 (C-13), 63.49 (C-14), 63.18 (Bn), 44.91 (C-3), 41.95 (C-7), 41.19 (C-6), 40.94 (C-8), 37.88 (C-9), 34.25 (C-5), 33.33 (C-15), 32.69 (C-2), 27.47 (C-12), 22.33 (C-19), 19.95 (C-20), 18.56 (C-16, C-17), 18.50 (C-18), 18.33 (C-16, C-17). IR (Diamond-ATR, neat) $\tilde{v}_{\text {max }} 3474$ (br), $2958(\mathrm{~s}), 2933(\mathrm{~s}), 2857(\mathrm{~m}), 1453(\mathrm{~m}), 1385(\mathrm{~m}), 1198(\mathrm{w}), 1087(\mathrm{~m}), 1055$ (s), $1027(\mathrm{~m})$, $1000(\mathrm{~m}) \mathrm{cm}^{-1}$. HRMS (ESI): calcd for ([M+CH$\left.\left.{ }_{3} \mathrm{COO}\right], \mathrm{C}_{29} \mathrm{H}_{41} \mathrm{O}_{5}\right)^{-}$: 469.2954, found: 469.2962. $[\alpha]_{D}^{20}=-19.6^{\circ}\left(\mathrm{c}=0.21, \mathrm{CH}_{2} \mathrm{Cl}_{2}\right)$.

Enol ether 42: TLC (20\% ethyl acetate in hexanes): $\mathrm{R}_{f}=0.28$ (CAM). ${ }^{1} \mathrm{H}$ NMR $\left(400 \mathrm{MHz}, \mathrm{C}_{6} \mathrm{D}_{6}\right) \delta$ 7.45-7.39 (m, 2H, Ph), 7.26-7.21 (m, 2H, Ph), 7.14-7.08 (m, 1H, Ph), 4.57-4.55 (m, 1H, 20- $\left.\mathrm{H}_{\mathrm{A}}\right), 4.36-4.22$ $(\mathrm{m}, 2 \mathrm{H}, \mathrm{Bn}), 3.87\left(\mathrm{~d},{ }^{2} \mathrm{~J}_{20 \mathrm{~B} / 20 \mathrm{~A}}=1.3 \mathrm{~Hz}, 1 \mathrm{H}, 20-\mathrm{H}_{\mathrm{B}}\right), 3.23-3.13(\mathrm{~m}, 1 \mathrm{H}, 13-\mathrm{H}), 2.27\left(\mathrm{dd},{ }^{2} J_{2 \mathrm{~A} / 2 \mathrm{~B}}=14.5 \mathrm{~Hz}\right.$, $\left.{ }^{3} J_{2 \mathrm{~A} / 3}=3.1 \mathrm{~Hz}, 1 \mathrm{H}, 2-\mathrm{H}_{\mathrm{A}}\right), 2.09-1.98\left(\mathrm{~m}, 2 \mathrm{H}, 5-\mathrm{H}_{\mathrm{A}}, 15-\mathrm{H}\right), 1.93\left(\mathrm{dd},{ }^{2} J_{2 \mathrm{~B} / 2 \mathrm{~A}}=14.5 \mathrm{~Hz},{ }^{3} J_{2 \mathrm{~B} / 3}=12.9 \mathrm{~Hz}, 1 \mathrm{H}\right.$, 2- $\left.\mathrm{H}_{\mathrm{B}}\right), 1.79-1.71\left(\mathrm{~m}, 1 \mathrm{H}, 12-\mathrm{H}_{\mathrm{A}}\right), 1.69-1.63(\mathrm{~m}, 1 \mathrm{H}, 3-\mathrm{H}), 1.63-1.54\left(\mathrm{~m}, 1 \mathrm{H}, 5-\mathrm{H}_{\mathrm{B}}\right), 1.54-1.45\left(\mathrm{~m}, 3 \mathrm{H}, 6-\mathrm{H}_{\mathrm{A}}\right.$, $\left.11-\mathrm{H}_{\mathrm{A}}, 12-\mathrm{H}_{\mathrm{B}}\right), 1.40\left(\mathrm{dd},{ }^{2} \mathrm{~J}_{8 \mathrm{~A} / 8 \mathrm{~B}}=11.9 \mathrm{~Hz},{ }^{3} \mathrm{JAA}_{8 \mathrm{9}}=5.3 \mathrm{~Hz}, 1 \mathrm{H}, 8-\mathrm{H}_{\mathrm{A}}\right), 1.30\left(\mathrm{dd},{ }^{3} \mathrm{~J}_{9 / 8 \mathrm{~B}}=12.0 \mathrm{~Hz},{ }^{3} \mathrm{~J}_{9 / 8 \mathrm{~A}}=5.3 \mathrm{~Hz}\right.$, 
$1 \mathrm{H}, 9-\mathrm{H}), 1.18-1.08\left(\mathrm{~m}, 4 \mathrm{H}, 6-\mathrm{H}_{\mathrm{B}}, 8-\mathrm{H}_{\mathrm{B}}, 11-\mathrm{H}_{\mathrm{B}}, 13-\mathrm{OH}\right), 1.06\left(\mathrm{~d},{ }^{3} \mathrm{~J}=6.9 \mathrm{~Hz}, 3 \mathrm{H}, 16-\mathrm{H}, 17-\mathrm{H}\right), 1.02(\mathrm{~s}, 3 \mathrm{H}$, 19-H), $1.00(\mathrm{~s}, 3 \mathrm{H}, 18-\mathrm{H}), 0.89\left(\mathrm{~d},{ }^{3} \mathrm{~J}=6.7 \mathrm{~Hz}, 3 \mathrm{H}, 16-\mathrm{H}, 17-\mathrm{H}\right) .{ }^{13} \mathrm{C}$ NMR $\left(100 \mathrm{MHz}, \mathrm{C}_{6} \mathrm{D}_{6}\right) \delta 164.57(\mathrm{C}-14)$, $140.55(P h), 128.58(P h), 127.33(P h), 127.23(P h), 88.40$ (C-4), 83.11 (C-10), 78.75 (C-20), 74.36 (C-13), $63.15(B n), 55.24$ (C-1), 47.25 (C-9), 46.37 (C-3), 42.03 (C-7), 40.85 (C-6), 38.86 (C-8), 37.72 (C-11), 34.09 (C-5), 33.42 (C-15), 30.73 (C-12), 26.47 (C-2), 20.56 (C-19), 18.67 (C-18), 18.41 (C-16, C-17), 18.38 (C-16, C-17). IR (Diamond-ATR, neat) $\tilde{v}_{\max }: 3457$ (br), $2934(\mathrm{~s}), 2856(\mathrm{~m}), 1664(\mathrm{~m}), 1454(\mathrm{~m})$, $1379(\mathrm{~m}), 1219(\mathrm{w}), 1197(\mathrm{w}), 1087(\mathrm{~m}), 1060(\mathrm{~s}), 1028(\mathrm{~m}) \mathrm{cm}^{-1}$. HRMS (EI): calcd for ([M], $\left.\mathrm{C}_{27} \mathrm{H}_{38} \mathrm{O}_{3}\right)^{+}$: 410.2821, found: 410.2813 . $[\alpha]_{D}^{20}=-1.5^{\circ}\left(\mathrm{c}=0.27, \mathrm{CH}_{2} \mathrm{Cl}_{2}\right)$. 


\subsection{Synthesis of bromide 23}

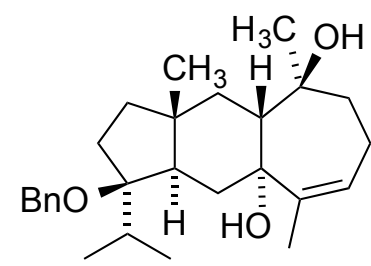

14

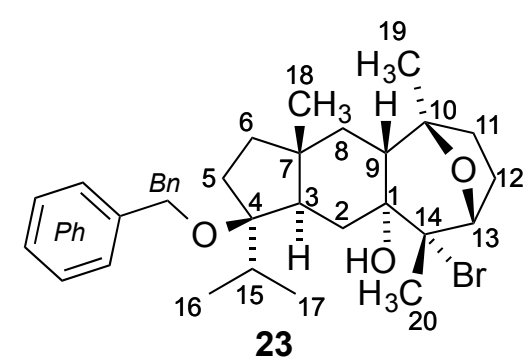

23

A solution of tricycle 14 (14.5 mg, $35.1 \mu \mathrm{mol}, 1$ equiv) in dichloromethane $(1.5 \mathrm{~mL})$ was treated with $\mathrm{N}$ bromosuccinimide $\left(6.9 \mathrm{mg}, 38.1 \mu \mathrm{mol}, 1.10\right.$ equiv) at $0{ }^{\circ} \mathrm{C}$. After $10 \mathrm{~min}$, the mixture was diluted with $\mathrm{pH} 7$ buffer solution $(15 \mathrm{~mL})$ and diethyl ether $(10 \mathrm{~mL})$. The layers were separated, the aqueous layer was extracted with diethyl ether $(3 \times 10 \mathrm{~mL})$ and the combined organic extracts were dried over sodium sulfate. The dried solution was filtered through a pad of Celite and the filtrate was concentrated to provide spectroscopically pure bromide $\mathbf{2 3}(17.3 \mathrm{mg}, \geq 99 \%)$ as a colourless oil.

TLC (5\% ethyl acetate in hexanes): $\mathrm{R}_{f}=0.34$ (CAM). ${ }^{1} \mathrm{H}$ NMR $\left(800 \mathrm{MHz}, \mathrm{C}_{6} \mathrm{D}_{6}\right) \delta 7.38-7.35(\mathrm{~m}, 2 \mathrm{H}, P h)$, 7.24-7.21 (m, 2H, Ph), 7.13-7.09 (m, 1H, Ph), 4.27-4.21 (m, 3H, 13-H, Bn), 2.78-2.73 (m, 1H, 12- $\left.\mathrm{H}_{\mathrm{A}}\right)$, 2.56-2.51 (m, $\left.1 \mathrm{H}, 11-\mathrm{H}_{\mathrm{A}}\right), 2.27\left(\mathrm{dd},{ }^{3} J_{3 / 2 \mathrm{~A}}=12.4 \mathrm{~Hz},{ }^{3} \mathrm{~J}_{3 / 2 \mathrm{~B}}=2.8 \mathrm{~Hz}, 1 \mathrm{H}, 3-\mathrm{H}\right), 2.10-2.07(\mathrm{~m}, 1 \mathrm{H}, 9-\mathrm{H})$, 2.06-2.00 (m, 2H, 5- $\left.\mathrm{H}_{\mathrm{A}}, 15-\mathrm{H}\right), 1.99-1.93\left(\mathrm{~m}, 1 \mathrm{H}, 12-\mathrm{H}_{\mathrm{B}}\right), 1.88\left(\mathrm{app} \mathrm{t},{ }^{2} J_{2 \mathrm{~A} / 2 \mathrm{~B}}={ }^{3} \mathrm{~J}_{2 \mathrm{~A} / 3}=12.8 \mathrm{~Hz}, 1 \mathrm{H}, 2-\mathrm{H}_{\mathrm{A}}\right)$, 1.81-1.78 (m, 4H, 2- $\left.\mathrm{H}_{\mathrm{B}}, 20-\mathrm{H}\right), 1.77\left(\mathrm{~d},{ }^{4} \mathrm{~J}_{1 \mathrm{OH} / 9}=1.9 \mathrm{~Hz}, 1 \mathrm{H}, 1-\mathrm{OH}\right), 1.65-1.60\left(\mathrm{~m}, 1 \mathrm{H}, 5-\mathrm{H}_{\mathrm{B}}\right), 1.59-1.51(\mathrm{~m}$, $\left.2 \mathrm{H}, 6-\mathrm{H}_{\mathrm{A}}, 8-\mathrm{H}_{\mathrm{A}}\right), 1.32-1.27\left(\mathrm{~m}, 2 \mathrm{H}, 8-\mathrm{H}_{\mathrm{B}}, 11-\mathrm{H}_{\mathrm{B}}\right), 1.19-1.13\left(\mathrm{~m}, 4 \mathrm{H}, 6-\mathrm{H}_{\mathrm{B}}, 19-\mathrm{H}\right), 1.06(\mathrm{~s}, 3 \mathrm{H}, 18-\mathrm{H}), 0.99(\mathrm{~d}$, $\left.{ }^{3} \mathrm{~J}=6.8 \mathrm{~Hz}, 3 \mathrm{H}, 16-\mathrm{H}, 17-\mathrm{H}\right), 0.93\left(\mathrm{~d},{ }^{3} \mathrm{~J}=6.8 \mathrm{~Hz}, 3 \mathrm{H}, 16-\mathrm{H}, 17-\mathrm{H}\right) .{ }^{13} \mathrm{C}$ NMR $\left(200 \mathrm{MHz}, \mathrm{C}_{6} \mathrm{D}_{6}\right) \delta 140.42(P h)$, $128.55(P h), 127.39(P h), 127.26(P h), 88.37$ (C-4), 85.99 (C-13), 83.99 (C-10, C-14), 83.89 (C-10, C-14), 76.48 (C-1), 63.27 (Bn), 47.53 (C-9), 44.58 (C-3), 42.74 (C-7), 40.65 (C-6), 38.14 (C-8), 34.01 (C-2), 33.89 (C-5), 32.97 (C-15), 31.87 (C-11), 30.28 (C-12), 28.93 (C-20), 25.54 (C-19), 19.69 (C-18), 18.67 (2C, 16-C, 17-C). IR (Diamond-ATR, neat) $\tilde{v}_{\text {max }}: 3547(\mathrm{~m}), 2937(\mathrm{~s}), 2869(\mathrm{~m}), 1452(\mathrm{~m}), 1377(\mathrm{~m}), 1276(\mathrm{~m})$, $1195(\mathrm{~m}), 1117(\mathrm{~m}), 1056(\mathrm{~s}), 1018(\mathrm{~s}) \mathrm{cm}^{-1}$. HRMS (ESI): calcd for ([M+CH $\left.\mathrm{CHOO}_{3}, \mathrm{C}_{29} \mathrm{H}_{42}{ }^{79} \mathrm{BrO}_{5}\right)^{-}$: 549.22156, found: 549.22233; calcd for $\left(\left[\mathrm{M}+\mathrm{CH}_{3} \mathrm{COO}\right], \mathrm{C}_{29} \mathrm{H}_{42}{ }^{81} \mathrm{BrO}_{5}\right)^{-}:$:551.21952, found: 551.22023. $[\alpha]_{D}^{20}=+62.3^{\circ}\left(\mathrm{c}=0.10, \mathrm{CH}_{2} \mathrm{Cl}_{2}\right)$. 


\subsection{Synthesis of nitrate ester 24}

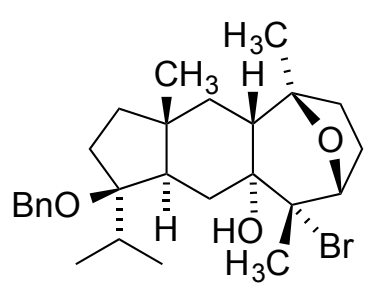

23

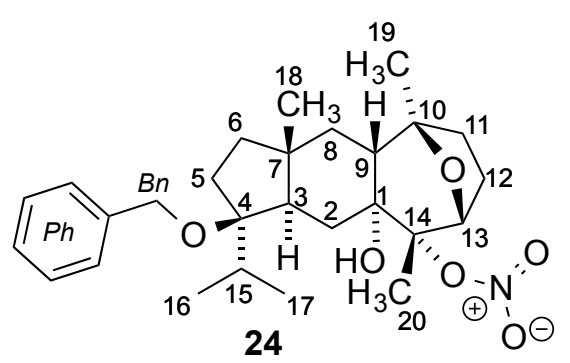

24

A solution of bromide 23 (17.3 mg, $35.1 \mu \mathrm{mol}, 1$ equiv) in acetone (1.5 mL) was treated with sodium hydrogen carbonate $(14.7 \mathrm{mg}, 175 \mu \mathrm{mol}, 5.00$ equiv) and silver nitrate $(17.9 \mathrm{mg}, 105 \mu \mathrm{mol}, 3.00$ equiv) at $23{ }^{\circ} \mathrm{C}$. Gradual formation of a greyish precipitate was observed. After $1 \mathrm{~h}$, the suspension was filtered through a pad of cotton wool and the filtrate was concentrated. The residue was purified by flash-column chromatography on silica gel (3\% ethyl acetate in hexanes) to provide nitrate ester 24 $(10.0 \mathrm{mg}, 60 \%)$ as a colourless oil.

TLC (5\% ethyl acetate in hexanes): $\mathrm{R}_{f}=0.20$ (CAM). ${ }^{1} \mathrm{H}$ NMR $\left(600 \mathrm{MHz}, \mathrm{C}_{6} \mathrm{D}_{6}\right) \delta 7.37-7.33(\mathrm{~m}, 2 \mathrm{H}, P h)$, 7.26-7.22 (m, 2H, Ph), 7.14-7.10 (m, $1 \mathrm{H}, P h), 4.87\left(\mathrm{~d},{ }^{3} J_{13 / 12 \mathrm{~B}}=7.7 \mathrm{~Hz}, 1 \mathrm{H}, 13-\mathrm{H}\right), 4.21$ (app s, 2H, Bn), 2.46-2.40 $\left(\mathrm{m}, 1 \mathrm{H}, 11-\mathrm{H}_{\mathrm{A}}\right), 2.39-2.33\left(\mathrm{~m}, 1 \mathrm{H}, 12-\mathrm{H}_{\mathrm{A}}\right), 2.07\left(\mathrm{dd},{ }^{3} J_{3 / 2 \mathrm{~A}}=12.5 \mathrm{~Hz},{ }_{3}^{3} J_{3 / 2 B}=2.8 \mathrm{~Hz}, 1 \mathrm{H}, 3-\mathrm{H}\right)$, 2.04-1.95 (m, 2H, 5- $\left.\mathrm{H}_{\mathrm{A}}, 15-\mathrm{H}\right), 1.84\left(\mathrm{dd},{ }^{3} \mathrm{~J}_{9 / 8 \mathrm{~A}}=13.0 \mathrm{~Hz},{ }^{3} \mathrm{~J}_{9 / 8 \mathrm{~B}}=3.3 \mathrm{~Hz}, 1 \mathrm{H}, 9-\mathrm{H}\right), 1.80-1.71\left(\mathrm{~m}, 2 \mathrm{H}, 2-\mathrm{H}_{\mathrm{A}}\right.$, $\left.12-\mathrm{H}_{\mathrm{B}}\right), 1.62\left(\mathrm{dd},{ }^{2} \mathrm{~J}_{2 \mathrm{~B} / 2 \mathrm{~A}}=13.5 \mathrm{~Hz},{ }^{3} \mathrm{~J}_{2 \mathrm{~B} / 3}=2.8 \mathrm{~Hz}, 1 \mathrm{H}, 2-\mathrm{H}_{\mathrm{B}}\right), 1.60-1.54\left(\mathrm{~m}, 1 \mathrm{H}, 5-\mathrm{H}_{\mathrm{B}}\right), 1.49-1.43(\mathrm{~m}, 2 \mathrm{H}$, $\left.6-\mathrm{H}_{\mathrm{A}}, 8-\mathrm{H}_{\mathrm{A}}\right), 1.39(\mathrm{br} \mathrm{s}, 1 \mathrm{H}, 1-\mathrm{OH}), 1.38(\mathrm{~s}, 3 \mathrm{H}, 20-\mathrm{H}), 1.31-1.24\left(\mathrm{~m}, 1 \mathrm{H}, 11-\mathrm{H}_{\mathrm{B}}\right), 1.22\left(\mathrm{dd},{ }^{2} \mathrm{~J}_{8 \mathrm{~B} / 8 \mathrm{~A}}=11.8 \mathrm{~Hz}\right.$, $\left.{ }^{3} J_{8 \mathrm{~B} / 9}=3.3 \mathrm{~Hz}, 1 \mathrm{H}, 8-\mathrm{H}_{\mathrm{B}}\right), 1.15(\mathrm{~s}, 3 \mathrm{H}, 19-\mathrm{H}), 1.12-1.05\left(\mathrm{~m}, 1 \mathrm{H}, 6-\mathrm{H}_{\mathrm{B}}\right), 1.00(\mathrm{~s}, 3 \mathrm{H}, 18-\mathrm{H}), 0.92\left(\mathrm{~d},{ }^{3} \mathrm{~J}=6.8 \mathrm{~Hz}\right.$, $3 \mathrm{H}, 16-\mathrm{H}, 17-\mathrm{H}), 0.87\left(\mathrm{~d},{ }^{3} \mathrm{~J}=6.8 \mathrm{~Hz}, 3 \mathrm{H}, 16-\mathrm{H}, 17-\mathrm{H}\right) .{ }^{13} \mathrm{C} \mathrm{NMR}\left(100 \mathrm{MHz}, \mathrm{C}_{6} \mathrm{D}_{6}\right) \delta 140.29(P h), 128.57(P h)$, $127.48(P h), 127.36(P h), 92.15$ (C-14), 88.21 (C-4), 83.26 (C-10), 79.65 (C-13), 76.77 (C-1), $63.18(B n)$, 47.12 (C-9), 44.34 (C-3), 42.30 (C-7), 40.57 (C-6), 36.92 (C-8), 33.79 (C-5), 32.97 (C-15), 32.44 (C-11), 32.36 (C-2), 28.17 (C-12), 25.27 (C-19), 20.28 (C-20), 19.51 (C-18), 18.60 (C-16, C-17), 18.56 (C-16, C-17). IR (Diamond-ATR, neat) $\tilde{v}_{\text {max }}: 3603(\mathrm{w}), 2937(\mathrm{~m}), 2865(\mathrm{~m}), 1627(\mathrm{~s}), 1453(\mathrm{~m}), 1381(\mathrm{~m}), 1290(\mathrm{~s})$, $1197(\mathrm{~m}), 1117(\mathrm{~m}), 1060(\mathrm{~m}) \mathrm{cm}^{-1}$. HRMS (ESI): calcd for ([M+CH$\left.\left.{ }_{3} \mathrm{COO}\right], \mathrm{C}_{29} \mathrm{H}_{42} \mathrm{NO}_{8}\right)^{-}:$532.29104, found: 532.29170. $[\alpha]_{D}^{20}=+42.0^{\circ}\left(\mathrm{c}=0.33, \mathrm{CH}_{2} \mathrm{Cl}_{2}\right)$. 


\subsection{Synthesis of mesylate 43}<smiles>CC(C)[C@]1(O)CC[C@]2(C)C[C@H]3[C@H](C)CC[C@@H](O)[C@H](C)[C@@]3(O)C[C@@]21C</smiles>

16

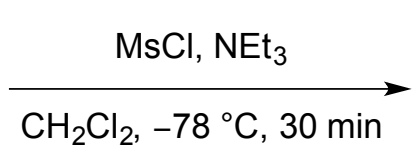

$(72 \%)$

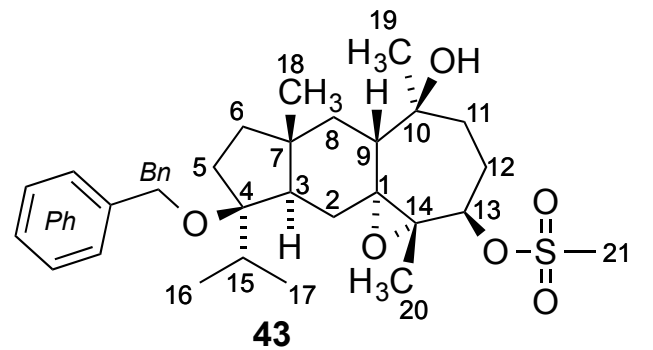

43

A solution of epoxide 16 (18.0 mg, $42.0 \mu \mathrm{mol}, 1$ equiv) in dichloromethane $(1.2 \mathrm{~mL})$ was treated sequentially with triethylamine $(23.3 \mu \mathrm{L}, 0.17 \mathrm{mmol}, 4.00$ equiv) and methanesulfonyl chloride $(6.5 \mu \mathrm{L}$, $84.0 \mu \mathrm{mol}, 2.00$ equiv) at $-78^{\circ} \mathrm{C}$. After $30 \mathrm{~min}$, the cooling bath was removed and the mixture was immediately diluted with $\mathrm{pH} 7$ buffer solution $(10 \mathrm{~mL})$ and ethyl acetate $(15 \mathrm{~mL})$. The layers were separated, the aqueous layer was extracted with ethyl acetate $(3 \times 15 \mathrm{~mL})$ and the combined organic extracts were dried over sodium sulfate. The dried solution was filtered and the filtrate was concentrated. The residue was purified by flash-column chromatography on silica gel $(50 \%$ ethyl acetate in hexanes) to provide mesylate $\mathbf{4 3}$ (15.3 $\mathrm{mg}, \mathbf{7 2} \%)$ as a colourless oil.

TLC (50\% ethyl acetate in hexanes): $\mathrm{R}_{f}=0.25$ (CAM). ${ }^{1} \mathrm{H}$ NMR $\left(800 \mathrm{MHz}, \mathrm{C}_{6} \mathrm{D}_{6}\right) \delta 7.33-7.30(\mathrm{~m}, 2 \mathrm{H}, \mathrm{Ph})$, 7.21-7.18 (m, 2H, Ph), 7.11-7.07 (m, 1H, Ph), $5.06\left(\mathrm{dd},{ }^{3} J_{13 / 12 \mathrm{~A}}=5.8 \mathrm{~Hz},{ }^{3} J_{13 / 12 \mathrm{~B}}=1.5 \mathrm{~Hz}, 1 \mathrm{H}, 13-\mathrm{H}\right)$, 4.23-4.16 (m, $2 \mathrm{H}, B n), 2.41\left(\mathrm{dd},{ }^{2} \mathrm{JAA}_{8 \mathrm{~B}}=12.8 \mathrm{~Hz},{ }^{3} \mathrm{~J}_{8 \mathrm{~A} / 9}=4.8 \mathrm{~Hz}, 1 \mathrm{H}, 8-\mathrm{H}_{\mathrm{A}}\right), 2.29-2.22\left(\mathrm{~m}, 2 \mathrm{H}, 2-\mathrm{H}_{\mathrm{A}}, 9-\mathrm{H}\right)$, $2.13(\mathrm{~s}, 3 \mathrm{H}, 21-\mathrm{H}), 2.04-1.94\left(\mathrm{~m}, 2 \mathrm{H}, 5-\mathrm{H}_{\mathrm{A}}, 15-\mathrm{H}\right), 1.94-1.84\left(\mathrm{~m}, 2 \mathrm{H}, 11-\mathrm{H}_{\mathrm{A}}, 12-\mathrm{H}_{\mathrm{A}}\right), 1.77$ (dd, $\left.{ }^{2} J_{2 \mathrm{~B} / 2 \mathrm{~A}}=14.9 \mathrm{~Hz},{ }^{3} \mathrm{~J}_{2 \mathrm{~B} / 3}=3.2 \mathrm{~Hz}, 1 \mathrm{H}, 2-\mathrm{H}_{\mathrm{B}}\right), 1.67\left(\mathrm{dd},{ }^{3} \mathrm{~J}_{3 / 2 \mathrm{~A}}=13.6 \mathrm{~Hz},{ }^{3} J_{3 / 2 \mathrm{~B}}=3.2 \mathrm{~Hz}, 1 \mathrm{H}, 3-\mathrm{H}\right), 1.62-1.57(\mathrm{~m}$, $\left.2 \mathrm{H}, 5-\mathrm{H}_{\mathrm{B}}, 6-\mathrm{H}_{\mathrm{A}}\right), 1.56-1.49\left(\mathrm{~m}, 2 \mathrm{H}, 8-\mathrm{H}_{\mathrm{B}}, 12-\mathrm{H}_{\mathrm{B}}\right), 1.40(\mathrm{~s}, 3 \mathrm{H}, 20-\mathrm{H}), 1.32-1.28\left(\mathrm{~m}, 1 \mathrm{H}, 11-\mathrm{H}_{\mathrm{B}}\right), 1.24(\mathrm{~s}, 3 \mathrm{H}$, $19-\mathrm{H}), 1.16-1.10\left(\mathrm{~m}, 1 \mathrm{H}, 6-\mathrm{H}_{\mathrm{B}}\right), 1.08(\mathrm{~s}, 3 \mathrm{H}, 18-\mathrm{H}), 0.93\left(\mathrm{~d},{ }^{3} \mathrm{~J}=6.8 \mathrm{~Hz}, 3 \mathrm{H}, 16-\mathrm{H}, 17-\mathrm{H}\right), 0.82\left(\mathrm{~d},{ }^{3} \mathrm{~J}=6.8 \mathrm{~Hz}\right.$, $3 \mathrm{H}, 16-\mathrm{H}, 17-\mathrm{H}) .{ }^{13} \mathrm{C}$ NMR $\left(200 \mathrm{MHz}, \mathrm{C}_{6} \mathrm{D}_{6}\right) \delta 140.32(P h), 128.58(P h), 127.31(P h), 127.26(P h)$, 88.27 (C-4), 87.56 (C-13), 74.28 (C-10), 66.18 (C-1), 63.82 (C-14), 63.19 (Bn), 48.20 (C-3), 45.64 (C-9), 41.72 (C-8), 41.34 (C-6), 41.12 (C-7), 38.23 (C-21), 38.15 (C-11), 34.52 (C-2), 33.61 (C-5), 33.20 (C-15), 25.43 (C-12), 23.90 (C-19), 22.36 (C-20), 18.50 (C-18), 18.26 (C-16, C-17), 17.84 (C16, C-17). IR (Diamond-ATR, neat) $\tilde{v}_{\max }: 3517$ (br), 2957 (s), 2929 (s), 1454 (m), 1353 (s), 1173 (s), 1087 (m), $1064(\mathrm{~m}), 970(\mathrm{w}), 898$ (s) $\mathrm{cm}^{-1}$. HRMS (ESI): calcd for ([M+ $\left.\left.\mathrm{CH}_{3} \mathrm{COO}\right], \mathrm{C}_{30} \mathrm{H}_{45} \mathrm{O}{ }_{8} \mathrm{~S}\right)^{-}:$565.28351, found: 565.28434. $[\alpha]_{D}^{20}=-9.4^{\circ}\left(\mathrm{c}=0.23, \mathrm{CH}_{2} \mathrm{Cl}_{2}\right)$. 


\subsection{Synthesis of epoxide-tetrahydrofuran 25}<smiles>CO[C@H]1CC[C@@](C)(O)[C@H]2C[C@]3(C)CC[C@](O)(C(C)C)[C@@H]3C[C@]2(O)[C@H]1C</smiles>

43

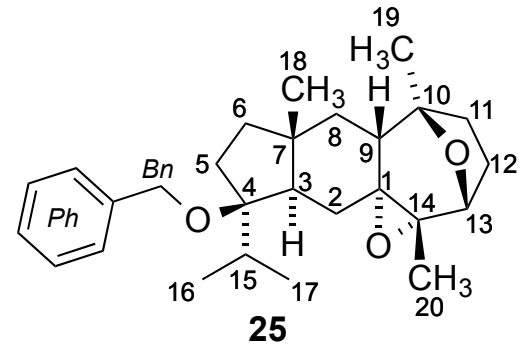

25

A solution of mesylate $43(2.0 \mathrm{mg}, 4.0 \mu \mathrm{mol}, 1$ equiv) in dimethyl formamide $(0.7 \mathrm{~mL})$ was treated sequentially with lithium bromide $(24.0 \mathrm{mg}, 0.28 \mathrm{mmol}, 70.0$ equiv) and lithium carbonate $(4.4 \mathrm{mg}$, $59.2 \mu \mathrm{mol}, 15.0$ equiv), and the resulting mixture was heated to $70{ }^{\circ} \mathrm{C}$. After $30 \mathrm{~min}$, the mixture was allowed to cool to $23{ }^{\circ} \mathrm{C}$, whereupon it was diluted with saturated aqueous sodium chloride solution $(10 \mathrm{~mL})$ and diethyl ether $(10 \mathrm{~mL})$. The layers were separated, the aqueous layer was extracted with diethyl ether $(3 \times 10 \mathrm{~mL})$ and the combined organic extracts were dried over sodium sulfate. The dried solution was filtered and the filtrate was concentrated. The residue was purified by flash-column chromatography on silica gel (10\% ethyl acetate in hexanes) to provide epoxide-tetrahydrofuran $\mathbf{2 5}$ (1.3 $\mathrm{mg}, 80 \%)$ as a colourless oil.

TLC (10\% ethyl acetate in hexanes): $\mathrm{R}_{f}=0.20$ (CAM). ${ }^{1} \mathrm{H}$ NMR (800 MHz, $\left.\mathrm{C}_{6} \mathrm{D}_{6}\right) \delta 7.39-7.36(\mathrm{~m}, 2 \mathrm{H}, P h)$, 7.30-7.27 (m, 2H, Ph), 7.16-7.15 (m, 1H, Ph), 4.27-4.21 (m, 2H, Bn), $4.17\left(\mathrm{~d},{ }^{3} J_{13 / 12 \mathrm{~B}}=6.4 \mathrm{~Hz}, 1 \mathrm{H}, 13-\mathrm{H}\right)$, 2.16-2.08 (m, 3H, 9-H, 11- $\left.\mathrm{H}_{\mathrm{A}}, 12-\mathrm{H}_{\mathrm{A}}\right), 2.05-1.96\left(\mathrm{~m}, 4 \mathrm{H}, 2-\mathrm{H}_{\mathrm{A}}, 3-\mathrm{H}, 5-\mathrm{H}_{\mathrm{A}}, 15-\mathrm{H}\right), 1.76-1.68\left(\mathrm{~m}, 1 \mathrm{H}, 12-\mathrm{H}_{\mathrm{B}}\right)$, $1.65-1.62\left(\mathrm{~m}, 1 \mathrm{H}, 2-\mathrm{H}_{\mathrm{B}}\right), 1.61-1.57\left(\mathrm{~m}, 1 \mathrm{H}, 5-\mathrm{H}_{\mathrm{B}}\right), 1.54\left(\mathrm{dd},{ }^{2} \mathrm{~J}_{6 \mathrm{~A} / 6 \mathrm{~B}}=11.6 \mathrm{~Hz},{ }^{3} \mathrm{~J}_{6 \mathrm{~A} / 5 \mathrm{~A}}=7.8 \mathrm{~Hz}, 1 \mathrm{H}, 6-\mathrm{H}_{\mathrm{A}}\right)$, $1.46\left(\mathrm{dd},{ }^{2} J_{8 \mathrm{~A} / 8 \mathrm{~B}}=11.8 \mathrm{~Hz},{ }^{3} \mathrm{~J}_{8 \mathrm{~A} / 9}=3.5 \mathrm{~Hz}, 1 \mathrm{H}, 8-\mathrm{H}_{\mathrm{A}}\right), 1.35-1.30\left(\mathrm{~m}, 1 \mathrm{H}, 8-\mathrm{H}_{\mathrm{B}}\right), 1.28(\mathrm{~s}, 3 \mathrm{H}, 19-\mathrm{H})$, 1.20-1.15 (m, 5H, 6- $\left.\mathrm{H}_{\mathrm{B}}, 11-\mathrm{H}_{\mathrm{B}}, 20-\mathrm{H}\right), 1.10(\mathrm{~s}, 3 \mathrm{H}, 18-\mathrm{H}), 0.96\left(\mathrm{~d},{ }^{3} \mathrm{~J}=6.8 \mathrm{~Hz}, 3 \mathrm{H}, 16-\mathrm{H}, 17-\mathrm{H}\right), 0.91(\mathrm{~d}$, $\left.{ }^{3} \mathrm{~J}=6.8 \mathrm{~Hz}, 3 \mathrm{H}, 16-\mathrm{H}, 17-\mathrm{H}\right) .{ }^{13} \mathrm{C}$ NMR $\left(200 \mathrm{MHz}, \mathrm{C}_{6} \mathrm{D}_{6}\right) \delta 140.56(P h), 128.50(P h), 127.19(P h)$, $127.13(P h), 87.68$ (C-4), 80.71 (C-10), 80.05 (C-13), 68.36 (C-14), 67.23 (C-1), 62.85 (Bn), 49.16 (C-3), 45.26 (C-9), 43.14 (C-7), 40.82 (C-6), 39.38 (C-8), 33.97 (C-5), 33.08 (C-15), 30.91 (C-11), 29.67 (C-12), 29.14 (C-2), 26.36 (C-19), 19.74 (C-18), 18.39 (C-16, C-17), 18.16 (C-16, C-17), 17.77 (C-20). IR (Diamond-ATR, neat) $\tilde{v}_{\text {max }} 2954(\mathrm{~s}), 1497(\mathrm{w}), 1453(\mathrm{~s}), 1376(\mathrm{~s}), 1263(\mathrm{~m}), 1199(\mathrm{~m}), 1137$ (m), $1085(\mathrm{~m}), 1061(\mathrm{~s}), 1036(\mathrm{~s}), 990(\mathrm{~m}) \mathrm{cm}^{-1}$. HRMS (EI): calcd for ([M-iPr], $\left.\mathrm{C}_{24} \mathrm{H}_{31} \mathrm{O}_{3}\right)^{+}: 367.2273$, found: 367.2264. $[\alpha]_{D}^{20}=+54.0^{\circ}\left(\mathrm{c}=0.10, \mathrm{CH}_{2} \mathrm{Cl}_{2}\right)$. 


\subsection{Synthesis of 5-6-6 tricycle 26}

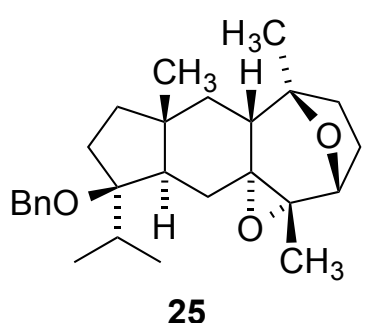

25 i. $\mathrm{Cu}\left(\mathrm{BF}_{4}\right)_{2} \cdot \mathrm{xH}_{2} \mathrm{O}$ $\mathrm{CH}_{2} \mathrm{Cl}_{2}, 40^{\circ} \mathrm{C}, 2 \mathrm{~h}$

ii. $\mathrm{H}_{2}, \mathrm{Pd} / \mathrm{C}$

$\mathrm{THF}, 23^{\circ} \mathrm{C}, 2 \mathrm{~h}$

$(90 \%)$

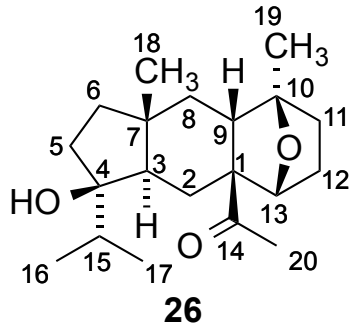

26

A solution of epoxide-tetrahydrofuran 25 (1.0 mg, $2.44 \mu \mathrm{mol}, 1$ equiv) in dichloromethane ( $1 \mathrm{~mL}$ ) was treated with copper(II) tetrafluoroborate hydrate $(1.2 \mathrm{mg}, 4.87 \mu \mathrm{mol}, 2.00$ equiv) and the resulting mixture was heated to $40{ }^{\circ} \mathrm{C}$. After $2 \mathrm{~h}$, the mixture was allowed to cool to $23{ }^{\circ} \mathrm{C}$, and was then diluted with $\mathrm{pH} 7$ buffer solution $(10 \mathrm{~mL})$ and diethyl ether $(10 \mathrm{~mL})$. The layers were separated, the aqueous layer was extracted with diethyl ether $(3 \times 10 \mathrm{~mL})$ and the combined organic extracts were dried over sodium sulfate. The dried solution was filtered and the filtrate was concentrated to provide the benzyl protected 5-6-6 tricycle which was used in the next step without further purification.

A solution of the benzyl ether (assuming $2.44 \mu \mathrm{mol})$ in tetrahydrofuran $(2 \mathrm{~mL})$ was treated with palladium on carbon ( $10 \mathrm{wt} . \%, 5.2 \mathrm{mg}, 4.87 \mu \mathrm{mol}, 2.00$ equiv) at $23^{\circ} \mathrm{C}$. An atmosphere of hydrogen was maintained by sparging the mixture with a stream of hydrogen gas using a stainless steel needle for $5 \mathrm{~min}$ and vigorous stirring of the suspension was then continued under hydrogen atmosphere at $23^{\circ} \mathrm{C}$. After $2 \mathrm{~h}$, the mixture was diluted with ethyl acetate $(10 \mathrm{~mL})$ and filtered through a short pad of Celite. The filtrate was concentrated and the residue was purified by flash-column chromatography on silica gel ( $40 \%$ ethyl acetate in hexanes) to provide 5-6-6 tricycle $\mathbf{2 6}(0.7 \mathrm{mg}, 90 \%$ over two steps) as a colourless oil.

TLC (40\% ethyl acetate in hexanes): $\mathrm{R}_{f}=0.22$ (CAM). ${ }^{1} \mathrm{H}$ NMR $\left(800 \mathrm{MHz}, \mathrm{C}_{6} \mathrm{D}_{6}\right) \delta 4.22\left(\mathrm{~d},{ }^{3} \mathrm{~J}_{13 / 12}=5.3 \mathrm{~Hz}\right.$, $1 \mathrm{H}, 13-\mathrm{H}), 3.29-3.25(\mathrm{~m}, 1 \mathrm{H}, 9-\mathrm{H}), 1.91(\mathrm{~s}, 3 \mathrm{H}, 20-\mathrm{H}), 1.85-1.77\left(\mathrm{~m}, 2 \mathrm{H}, 5-\mathrm{H}_{\mathrm{A}}, 11-\mathrm{H}_{\mathrm{A}}\right), 1.69$ (dd, $\left.{ }^{2} J_{8 \mathrm{~A} / 8 \mathrm{~B}}=12.8 \mathrm{~Hz},{ }^{3} J_{8 \mathrm{~A} / 9}=10.4 \mathrm{~Hz}, 1 \mathrm{H}, 8-\mathrm{H}_{\mathrm{A}}\right), 1.63-1.56\left(\mathrm{~m}, 3 \mathrm{H}, 2-\mathrm{H}_{\mathrm{A}}, 12-\mathrm{H}\right), 1.50-1.44\left(\mathrm{~m}, 2 \mathrm{H}, 5-\mathrm{H}_{\mathrm{B}}, 6-\mathrm{H}_{\mathrm{A}}\right)$, 1.43-1.38 (m, 1H, 15-H), 1.38-1.35 (m, $\left.1 \mathrm{H}, 2-\mathrm{H}_{\mathrm{B}}\right), 1.34(\mathrm{~s}, 3 \mathrm{H}, 19-\mathrm{H}), 1.17-1.08\left(\mathrm{~m}, 2 \mathrm{H}, 3-\mathrm{H}, 11-\mathrm{H}_{\mathrm{B}}\right)$, $1.00(\mathrm{~s}, 3 \mathrm{H}, 18-\mathrm{H}), 0.94-0.88\left(\mathrm{~m}, 1 \mathrm{H}, 6-\mathrm{H}_{\mathrm{B}}\right), 0.84\left(\mathrm{~d},{ }^{3} \mathrm{~J}=6.8 \mathrm{~Hz}, 3 \mathrm{H}, 16-\mathrm{H}, 17-\mathrm{H}\right), 0.83-0.80\left(\mathrm{~m}, 1 \mathrm{H}, 8-\mathrm{H}_{\mathrm{B}}\right)$, $0.78\left(\mathrm{~d},{ }^{3} \mathrm{~J}=6.8 \mathrm{~Hz}, 3 \mathrm{H}, 16-\mathrm{H}, 17-\mathrm{H}\right), 0.52$ (br s, $\left.1 \mathrm{H}, 4-\mathrm{OH}\right) .{ }^{13} \mathrm{C}$ NMR $\left(200 \mathrm{MHz}, \mathrm{C}_{6} \mathrm{D}_{6}\right) \delta 206.79(\mathrm{C}-14)$, 88.78 (C-10), 82.99 (C-13), 82.69 (C-4), 59.57 (C-1), 49.25 (C-3), 41.92 (C-7), 41.15 (C-9), 40.50 (C-6), 38.96 (C-8), 37.90 (C-15), 37.64 (C-5), 30.51 (C-11), 26.02 (C-12), 24.86 (C-20), 23.86 (C-2), 20.89 (C-19), 19.69 (C-18), 18.40 (C-16, C-17), 17.80 (C-16, C-17). IR (Diamond-ATR, neat) $\tilde{v}_{\max }: 3499$ (br), 2958 (s), $2872(\mathrm{~m}), 1705(\mathrm{~s}), 1469(\mathrm{~m}), 1382(\mathrm{~m}), 1281(\mathrm{w}), 1197(\mathrm{~m}), 998(\mathrm{~m}) \mathrm{cm}^{-1}$. HRMS (ESI): calcd for ([M+H], $\left.\mathrm{C}_{20} \mathrm{H}_{33} \mathrm{O}_{3}\right)^{+}: 321.24297$, found: 321.24329 .

$[\alpha]_{D}^{20}=-29.1^{\circ}\left(\mathrm{c}=0.12, \mathrm{CH}_{2} \mathrm{Cl}_{2}\right)$. 


\subsection{Synthesis of silyl ether 44}
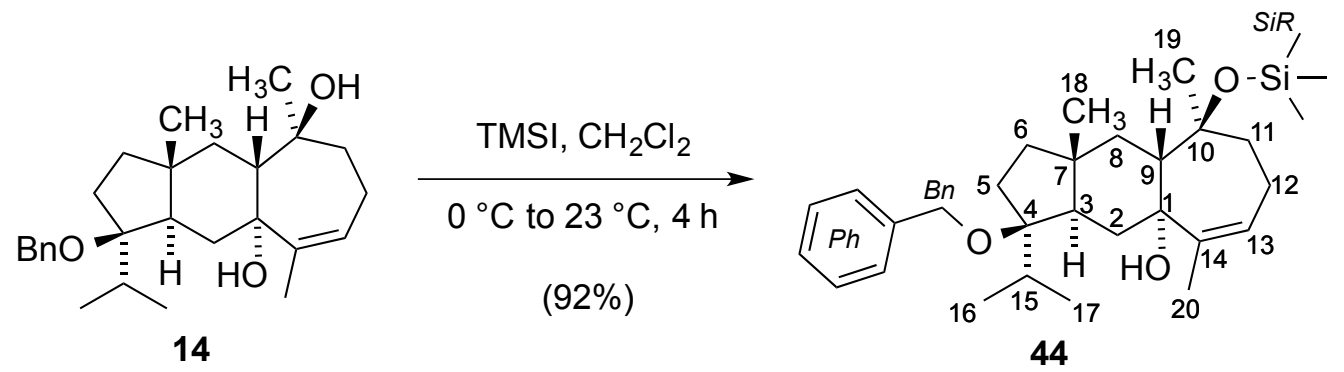

A solution of tricycle 14 (140 mg, $339 \mu \mathrm{mol}, 1$ equiv) in dichloromethane $(1.5 \mathrm{~mL})$ was treated with $\mathrm{N}$-trimethylsilylimidazole $\left(0.50 \mathrm{~mL}, 3.39 \mathrm{mmol}, 10.0\right.$ equiv) at $0{ }^{\circ} \mathrm{C}$. After $5 \mathrm{~min}$, the mixture was warmed to $23{ }^{\circ} \mathrm{C}$. After $4 \mathrm{~h}$, the mixture was diluted with $\mathrm{pH} 7$ buffer solution $(35 \mathrm{~mL})$ and diethyl ether $(20 \mathrm{~mL})$. The layers were separated, the aqueous layer was extracted with diethyl ether $(3 \times 15 \mathrm{~mL})$ and the combined organic extracts were dried over sodium sulfate. The dried solution was filtered and the filtrate was concentrated. The residue was purified by flash-column chromatography on silica gel (3\% ethyl acetate in hexanes) to provide silyl ether $\mathbf{4 4}(151 \mathrm{mg}, 92 \%)$ as a colourless oil.

TLC (10\% ethyl acetate in hexanes): $\mathrm{R}_{f}=0.49$ (CAM). ${ }^{1} \mathrm{H}$ NMR (400 MHz, $\left.\mathrm{C}_{6} \mathrm{D}_{6}\right) \delta 7.37-7.31(\mathrm{~m}, 2 \mathrm{H}, \mathrm{Ph})$, 7.22-7.17 (m, 2H, Ph), 7.12-7.06 (m, 1H, Ph), 5.66-5.60 (m, 1H, 13-H), 4.30-4.21 (m, 2H, Bn), $2.55-2.43\left(\mathrm{~m}, 1 \mathrm{H}, 12-\mathrm{H}_{\mathrm{A}}\right), 2.34\left(\mathrm{dd},{ }^{3} \mathrm{~J}_{9 / 8 \mathrm{~B}}=12.5 \mathrm{~Hz},{ }^{3} \mathrm{~J}_{9 / 8 \mathrm{~A}}=3.8 \mathrm{~Hz}, 1 \mathrm{H}, 9-\mathrm{H}\right), 2.23\left(\mathrm{dd},{ }^{2} \mathrm{~J}_{8 \mathrm{~A} / 8 \mathrm{~B}}=12.2 \mathrm{~Hz}\right.$, $\left.{ }^{3} J_{8 \mathrm{~A} / 9}=3.8 \mathrm{~Hz}, 1 \mathrm{H}, 8-\mathrm{H}_{\mathrm{A}}\right), 2.17\left(\mathrm{~d}^{2} \mathrm{~J}_{2 \mathrm{~A} / 2 \mathrm{~B}}=11.5 \mathrm{~Hz}, 1 \mathrm{H}, 2-\mathrm{H}_{\mathrm{A}}\right), 2.10-1.99\left(\mathrm{~m}, 2 \mathrm{H}, 5-\mathrm{H}_{\mathrm{A}}, 15-\mathrm{H}\right), 1.95-1.86(\mathrm{~m}$, $\left.3 \mathrm{H}, 2-\mathrm{H}_{B}, 3-\mathrm{H}, 11-\mathrm{H}_{A}\right), 1.81-1.57\left(\mathrm{~m}, 8 \mathrm{H}, 5-\mathrm{H}_{B}, 6-\mathrm{H}_{A}, 8-\mathrm{H}_{B}, 11-\mathrm{H}_{B}, 12-\mathrm{H}_{B}, 20-\mathrm{H}\right), 1.46(\mathrm{~s}, 3 \mathrm{H}, 19-\mathrm{H})$, $1.32-1.20\left(\mathrm{~m}, 1 \mathrm{H}, 6-\mathrm{H}_{\mathrm{B}}\right), 1.18(\mathrm{~s}, 3 \mathrm{H}, 18-\mathrm{H}), 1.03\left(\mathrm{~d},{ }^{3} \mathrm{~J}=6.8 \mathrm{~Hz}, 3 \mathrm{H}, 16-\mathrm{H}, 17-\mathrm{H}\right), 0.94\left(\mathrm{~d},{ }^{3} \mathrm{~J}=6.7 \mathrm{~Hz}, 3 \mathrm{H}\right.$, 16-H, 17-H), 0.86 (br s, 1H, 1-OH), 0.17 (s, 9H, SiR). $\left.{ }^{13} \mathrm{C} \mathrm{NMR} \mathrm{(100} \mathrm{MHz,} \mathrm{C}_{6} \mathrm{D}_{6}\right) \delta 141.15(\mathrm{C}-14), 140.62(\mathrm{Ph})$, 131.16 (C-13), $128.49(P h), 127.11(P h), 127.08(P h), 88.69$ (C-4), 79.83 (C-10), 76.72 (C-1), $63.03(B n)$, 47.81 (C-9), 45.96 (C-3), 45.83 (C-11), 41.66 (C-6, C-17), 41.53 (C-6, C-7), 39.78 (C-8), 38.52 (C-2), 34.25 (C-5), 33.21 (C-15), 28.43 (C-19), 22.42 (C-12), 21.39 (C-20), 18.70 (C-18), 18.63 (C-16, C-17), 18.39 (C-16, C-17), 2.86 (SiR). IR (Diamond-ATR, neat) $\tilde{v}_{\max }: 3464$ (br), 2955 (s), 2857 (m), 1453 (m), $1379(\mathrm{~m}), 1249(\mathrm{~m}), 1151(\mathrm{w}), 1105(\mathrm{~m}), 1086(\mathrm{~m}), 1027$ (s) $\mathrm{cm}^{-1}$. HRMS (EI): calcd for ([M- $\left.\mathrm{H}_{2} \mathrm{O}\right]$, $\left.\mathrm{C}_{30} \mathrm{H}_{46} \mathrm{O}_{2} \mathrm{Si}\right)^{+}: 466.3267$, found: 466.3249 . $[\alpha]_{D}^{20}=+12.8^{\circ}$ (c $=0.86, \mathrm{CH}_{2} \mathrm{Cl}_{2}$ ). 


\subsection{Synthesis of allylic alcohol 28}

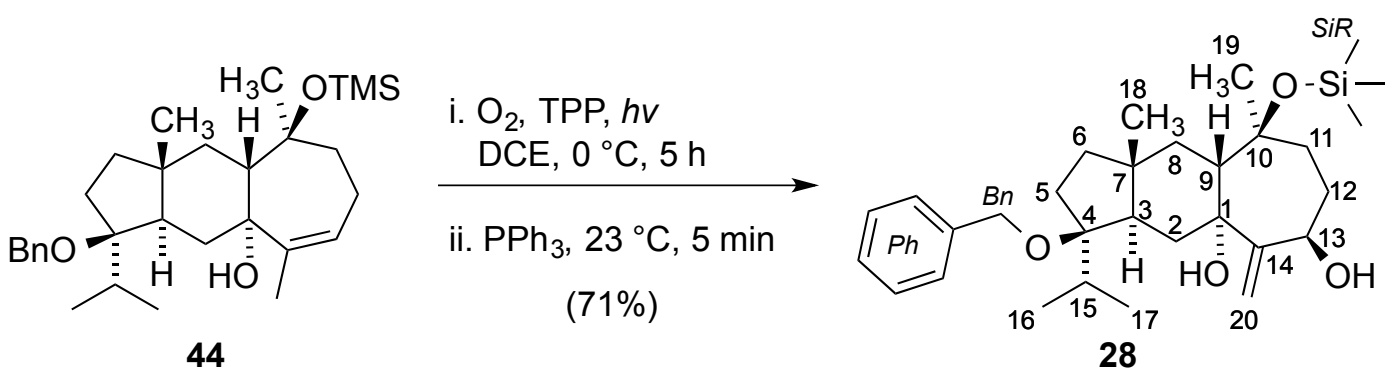

A stream of pure oxygen gas was bubbled below the liquid surface of a solution of silyl ether $\mathbf{4 4}$ (75.0 $\mathrm{mg}$, $155 \mu \mathrm{mol}, 1$ equiv) in dichloroethane $(20 \mathrm{~mL})$ containing a catalytic amount of tetraphenylporphyrin (TPP, tip of a spatula) at $0{ }^{\circ} \mathrm{C}$. After $3 \mathrm{~min}$, the mixture was irradiated with a Replux Belgium RL $160 \mathrm{~W}$ (225-235 Volts) lamp. After $5 \mathrm{~h}$, TLC analysis (20\% ethyl acetate in hexanes) indicated complete conversion of silyl ether $44\left(R_{f}=0.80\right)$ to the hydroperoxide intermediate $\left(R_{f}=0.29\right)$. Sparging with oxygen and irradiation was discontinued, triphenylphosphine $(81.2 \mathrm{mg}, 309 \mu \mathrm{mol}, 2.00$ equiv) was added and the mixture was then allowed to warm to $23{ }^{\circ} \mathrm{C}$. After $5 \mathrm{~min}$, TLC analysis ( $20 \%$ ethyl acetate in hexanes) indicated complete reduction of the hydroperoxide intermediate and formation of allylic alcohol $28\left(R_{f}=0.23\right)$. The mixture was concentrated in vacuo, and the residue was purified by flashcolumn chromatography on silica gel $(10 \% \rightarrow 20 \%$ ethyl acetate in hexanes) to provide allylic alcohol 28 (55.0 $\mathrm{mg}, 71 \%)$ as a colourless foam.

TLC (20\% ethyl acetate in hexanes): $\mathrm{R}_{f}=0.23$ (CAM). ${ }^{1} \mathrm{H}$ NMR $\left(800 \mathrm{MHz}, \mathrm{C}_{6} \mathrm{D}_{6}\right) \delta 7.39-7.36(\mathrm{~m}, 2 \mathrm{H}, \mathrm{Ph})$, 7.22-7.18 $(\mathrm{m}, 2 \mathrm{H}, P h), 7.10-7.07(\mathrm{~m}, 1 \mathrm{H}, \mathrm{Ph}), 5.17-5.11\left(\mathrm{~m}, 1 \mathrm{H}, 20-\mathrm{H}_{\mathrm{A}}\right), 4.74\left(\mathrm{br} s, 1 \mathrm{H}, 20-\mathrm{H}_{\mathrm{B}}\right)$, 4.29-4.23 (m, $2 \mathrm{H}, B n), 4.21-4.18(\mathrm{~m}, 1 \mathrm{H}, 13-\mathrm{H}), 2.64\left(\mathrm{br} \mathrm{d},{ }^{3} J_{9 / 8 \mathrm{~B}}=12.6 \mathrm{~Hz}, 1 \mathrm{H}, 9-\mathrm{H}\right), 2.40-2.35(\mathrm{~m}, 1 \mathrm{H}$, $\left.2-\mathrm{H}_{\mathrm{A}}\right), 2.12-2.03\left(\mathrm{~m}, 3 \mathrm{H}, 5-\mathrm{H}_{\mathrm{A}}, 8-\mathrm{H}_{\mathrm{A}}, 15-\mathrm{H}\right), 1.97\left(\mathrm{dd},{ }^{3} \mathrm{~J}_{3 / 2 \mathrm{~A}}=13.2 \mathrm{~Hz},{ }^{3} \mathrm{~J}_{3 / 2 \mathrm{~B}}=2.9 \mathrm{~Hz}, 1 \mathrm{H}, 3-\mathrm{H}\right), 1.93-1.89(\mathrm{~m}$, $2 \mathrm{H}, 12-\mathrm{H}), 1.70-1.57\left(\mathrm{~m}, 6 \mathrm{H}, 2-\mathrm{H}_{B}, 5-\mathrm{H}_{B}, 6-\mathrm{H}_{\mathrm{A}}, 8-\mathrm{H}_{\mathrm{B}}, 11-\mathrm{H}\right), 1.35(\mathrm{~s}, 3 \mathrm{H}, 19-\mathrm{H}), 1.32(\mathrm{~s}, 3 \mathrm{H}, 18-\mathrm{H})$, $1.28-1.22\left(\mathrm{~m}, 1 \mathrm{H}, 6-\mathrm{H}_{\mathrm{B}}\right), 1.06(\mathrm{br} \mathrm{s}, 1 \mathrm{H}, 1-\mathrm{OH}), 1.04\left(\mathrm{~d},{ }^{3} \mathrm{~J}=6.8 \mathrm{~Hz}, 3 \mathrm{H}, 16-\mathrm{H}, 17-\mathrm{H}\right), 0.95\left(\mathrm{~d},{ }^{3} \mathrm{~J}=6.7 \mathrm{~Hz}, 3 \mathrm{H}\right.$, $16-\mathrm{H}, 17-\mathrm{H}), 0.80-0.77(\mathrm{~m}, 1 \mathrm{H}, 13-\mathrm{OH}), 0.16(\mathrm{~s}, 9 \mathrm{H}, \mathrm{SiR}) .{ }^{13} \mathrm{C} N M R\left(200 \mathrm{MHz}, \mathrm{C}_{6} \mathrm{D}_{6}\right) \delta 160.21(\mathrm{C}-14)$, 140.49 (Ph), $128.52(P h), 127.65(P h), 127.22(P h), 111.37$ (C-20), 88.65 (C-4), 79.35 (C-10), 76.91 (C-1), 75.47 (br, C-13), 63.39 (Bn), 46.82 (2C, C-3, C-9), 43.68 (br, C-2), 42.81 (C-7), 41.16 (C-6), 39.29 (C-11), 37.85 (C-8), 34.08 (C-5), 33.23 (C-15), 29.82 (C-12), 26.99 (C-18), 19.42 (C-19), 18.72 (C-16, C-17), 18.59 (C-16, C-17), 2.91 (SiR). IR (Diamond-ATR, neat) $\tilde{v}_{\max }: 3463(\mathrm{br}), 2953(\mathrm{~s}), 2869$ (m), 1453 (m), $1385(\mathrm{~m}), 1248(\mathrm{~s}), 1118(\mathrm{~m}), 1041(\mathrm{~s}) \mathrm{cm}^{-1}$. HRMS (EI): calcd for ([M-iPr], $\left.\mathrm{C}_{27} \mathrm{H}_{41} \mathrm{O}_{4} \mathrm{Si}\right)^{+}:$457.2774, found: 457.2781. $[\alpha]_{D}^{20}=+28.5^{\circ}\left(\mathrm{c}=0.83, \mathrm{CH}_{2} \mathrm{Cl}_{2}\right.$ ). 


\subsection{Synthesis of oxetane 29}

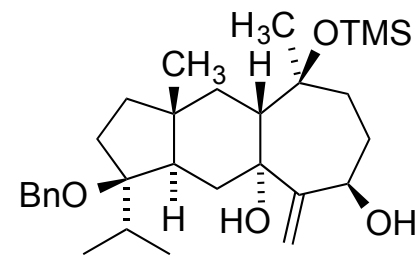

28 i. $\mathrm{MsCl}, \mathrm{NEt}_{3}$ $\mathrm{CH}_{2} \mathrm{Cl}_{2},-78^{\circ} \mathrm{C}, 1 \mathrm{~h}$

ii. $\mathrm{NaH}, \mathrm{THF}, 66^{\circ} \mathrm{C}, 1.5 \mathrm{~h}$

$(88 \%)$

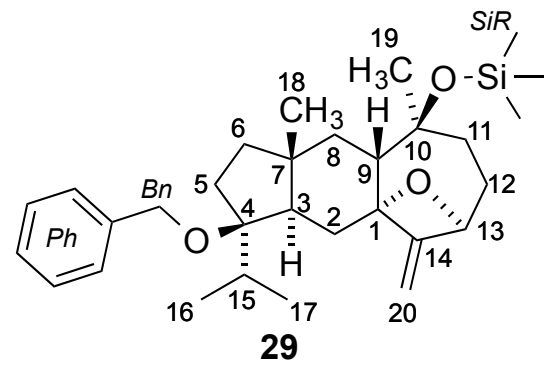

29

A solution of allylic alcohol $28(27.0 \mathrm{mg}, 53.9 \mu \mathrm{mol}, 1$ equiv) in dichloromethane $(2.5 \mathrm{~mL})$ was treated sequentially with triethylamine ( $172 \mu \mathrm{L}, 1.24 \mathrm{mmol}, 23.0$ equiv) and methanesulfonyl chloride ( $41.7 \mu \mathrm{L}$, $0.54 \mathrm{mmol}, 10.0$ equiv) at $-78{ }^{\circ} \mathrm{C}$. After $1 \mathrm{~h}$, the cooling bath was removed and the mixture was immediately diluted with saturated aqueous sodium hydrogen carbonate solution $(25 \mathrm{~mL})$ and diethyl ether $(15 \mathrm{~mL})$. The layers were separated, the aqueous layer was extracted with diethyl ether $(3 \times 15 \mathrm{~mL})$ and the combined organic extracts were dried over sodium sulfate. The dried solution was filtered and the filtrate was concentrated to provide the crude allylic mesylate which was used in the next step without further purification.

A solution of the crude allylic mesylate (assuming $53.9 \mu \mathrm{mol}$ ) in tetrahydrofuran $(4 \mathrm{~mL}$ ) was treated with sodium hydride ( $60 \mathrm{wt} . \%$ in mineral oil, $10.8 \mathrm{mg}, 0.27 \mathrm{mmol}, 5.00$ equiv) at $23{ }^{\circ} \mathrm{C}$. The resulting mixture was heated to $66^{\circ} \mathrm{C}$, whereupon a further portion of sodium hydride (60 wt.\% in mineral oil, $10.8 \mathrm{mg}$, $0.27 \mathrm{mmol}, 5.00$ equiv) was added. After $1.5 \mathrm{~h}$, the mixture was cooled to $0{ }^{\circ} \mathrm{C}$ and carefully diluted with saturated aqueous sodium hydrogen carbonate solution $(15 \mathrm{~mL})$ and diethyl ether $(15 \mathrm{~mL})$. The layers were separated, the aqueous layer was extracted with diethyl ether $(3 \times 15 \mathrm{~mL})$ and the combined organic extracts were dried over sodium sulfate. The dried solution was filtered and the filtrate was concentrated. The residue was purified by flash-column chromatography on silica gel (3\% ethyl acetate in hexanes) to provide oxetane $\mathbf{2 9}(23.0 \mathrm{mg}, 88 \%$ over two steps) as a colourless oil.

TLC (10\% ethyl acetate in hexanes): $\mathrm{R}_{f}=0.50$ (CAM). Note: Due to hindered rotation of the C10-OTMS group, oxetane 29 shows severe signal broadening in NMR spectra recorded at room temperature. Better resolved spectra could be obtained at $65^{\circ} \mathrm{C}$. However, some carbon atoms are still not visible in the ${ }^{13} \mathrm{C}$ NMR spectrum due to signal broadening. Most protons could therefore also not be assigned.

${ }^{1} \mathrm{H}$ NMR $\left(400 \mathrm{MHz}, \mathrm{C}_{6} \mathrm{D}_{6}, 65^{\circ} \mathrm{C}\right) \delta$ 7.37-7.33 (m, 2H, Ph), 7.22-7.17 (m, 2H, Ph), 7.11-7.05 (m, 1H, Ph), 4.96-4.91 (m, 1H, 13-H), $4.65\left(\mathrm{dd}, J=2.0 \mathrm{~Hz}, 1.2 \mathrm{~Hz}, 1 \mathrm{H}, 20-\mathrm{H}_{\mathrm{A}}\right), 4.47\left(\mathrm{app} \mathrm{t}, J=1.2 \mathrm{~Hz}, 1 \mathrm{H}, 20-\mathrm{H}_{\mathrm{B}}\right), 4.35-$ $4.26(\mathrm{~m}, 2 \mathrm{H}, B n), 2.33(\mathrm{dd}, J=14.1 \mathrm{~Hz}, 3.2 \mathrm{~Hz}, 1 \mathrm{H}), 2.26-2.17(\mathrm{~m}, 1 \mathrm{H}), 2.14-2.04(\mathrm{~m}, 4 \mathrm{H}), 2.00-1.82(\mathrm{~m}$, $3 \mathrm{H}), 1.72-1.50(\mathrm{~m}, 5 \mathrm{H}), 1.42(\mathrm{br} \mathrm{s}, 3 \mathrm{H}, 19-\mathrm{H}), 1.25-1.17(\mathrm{~m}, 1 \mathrm{H}), 1.15(\mathrm{~s}, 3 \mathrm{H}, 18-\mathrm{H}), 1.04\left(\mathrm{~d},{ }^{3} \mathrm{~J}=6.8 \mathrm{~Hz}\right.$, $3 \mathrm{H}, 16-\mathrm{H}, 17-\mathrm{H}), 0.93\left(\mathrm{~d},{ }^{3} \mathrm{~J}=6.8 \mathrm{~Hz}, 3 \mathrm{H}, 16-\mathrm{H}, 17-\mathrm{H}\right), 0.16(\mathrm{~s}, 9 \mathrm{H}, \mathrm{SiR}) .{ }^{13} \mathrm{C}$ NMR $\left(101 \mathrm{MHz}, \mathrm{C}_{6} \mathrm{D}_{6}, 65{ }^{\circ} \mathrm{C}\right) \delta$ 156.66, 140.66, 128.51, 127.47, 127.17, 99.29, 88.61, 84.25, 63.57, 51.60, 45.44, 42.29, 41.41, 39.61, 37.06, 34.14, 33.39, 29.93, 18.99, 18.40, 18.27, 2.98. IR (Diamond-ATR, neat) $\tilde{v}_{\text {max }} 2955$ (s), 1454 (m), $1374(\mathrm{~m}), 1349(\mathrm{~m}), 1248$ (s), 1159 (w), 1110 (s), 1084 (s), 1057 (s), 1027 (s) cm ${ }^{-1}$. HRMS (EI): calcd for ([M], $\left.\mathrm{C}_{30} \mathrm{H}_{46} \mathrm{O}_{3} \mathrm{Si}\right)^{+}: 482.3216$, found: 482.3211 . $[\alpha]_{D}^{20}=+4.2^{\circ}\left(\mathrm{c}=0.30, \mathrm{CH}_{2} \mathrm{Cl}_{2}\right.$ ). 


\subsection{Synthesis of iodide 30}

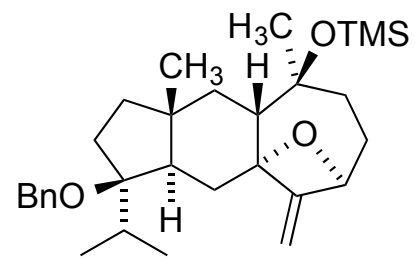

29

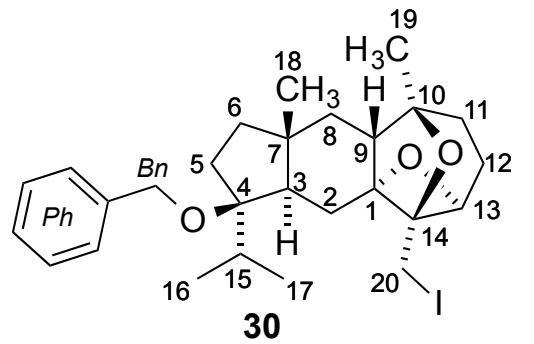

30

A solution of oxetane 29 ( $22.0 \mathrm{mg}, 45.6 \mu \mathrm{mol}, 1$ equiv) in dichloromethane $(2 \mathrm{~mL})$ was treated with $N$ iodosuccinimide ( $12.3 \mathrm{mg}, 54.7 \mu \mathrm{mol}, 1.20$ equiv) and the resulting pink mixture was stirred at $23{ }^{\circ} \mathrm{C}$ under exclusion of light. After $1.5 \mathrm{~h}, \mathrm{TLC}$ analysis indicated incomplete conversion of oxetane 29, and therefore a further portion of $\mathrm{N}$-iodosuccinimide ( $3.1 \mathrm{mg}, 13.7 \mu \mathrm{mol}, 0.30$ equiv) was added. After $1 \mathrm{~h}$, the mixture was diluted with saturated aqueous sodium hydrogen carbonate solution $(15 \mathrm{~mL})$ and diethyl ether $(10 \mathrm{~mL})$. The layers were separated, the aqueous layer was extracted with diethyl ether $(3 \times 10 \mathrm{~mL})$ and the combined organic extracts were dried over sodium sulfate. The dried solution was filtered through a pad of Celite and the filtrate was concentrated (under exclusion of light) to provide the crude iodide $\mathbf{3 0}$ which was used in the next step without further pruficiation.

An analytically pure sample of iodide $\mathbf{3 0}$ could be obtained by rapid purification by flash-column chromatography on silica gel (10\% ethyl acetate in hexanes) in the dark.

TLC (10\% ethyl acetate in hexanes): $\mathrm{R}_{f}=0.43$ (CAM). ${ }^{1} \mathrm{H}$ NMR $\left(800 \mathrm{MHz}, \mathrm{C}_{6} \mathrm{D}_{6}\right) \delta 7.42-7.38(\mathrm{~m}, 2 \mathrm{H}, \mathrm{Ph})$, 7.28-7.24 (m, 2H, Ph), 7.14-7.10 (m, 1H,Ph), 4.29-4.24 (m, 2H, Bn), $4.21\left(\mathrm{~d},{ }^{3} J_{13 / 12 \mathrm{~B}}=3.9 \mathrm{~Hz}, 1 \mathrm{H}, 13-\mathrm{H}\right)$, $3.04\left(\mathrm{~d},{ }^{2} J_{20 \mathrm{~A} / 20 \mathrm{~B}}=10.6 \mathrm{~Hz}, 1 \mathrm{H}, 20-\mathrm{H}_{\mathrm{A}}\right), 2.90\left(\mathrm{~d},{ }^{2} J_{20 \mathrm{~B} / 20 \mathrm{~A}}=10.6 \mathrm{~Hz}, 1 \mathrm{H}, 20-\mathrm{H}_{\mathrm{B}}\right), 2.15-2.12(\mathrm{~m}, 1 \mathrm{H}, 3-\mathrm{H})$, 2.12-2.07 (m, $\left.1 \mathrm{H}, 5-\mathrm{H}_{\mathrm{A}}\right), 2.06-2.02(\mathrm{~m}, 1 \mathrm{H}, 15-\mathrm{H}), 1.97\left(\mathrm{dd},{ }^{2} J_{2 \mathrm{~A} / 2 \mathrm{~B}}=12.5 \mathrm{~Hz},{ }^{3} J_{2 \mathrm{~A} / 3}=2.7 \mathrm{~Hz}, 1 \mathrm{H}, 2-\mathrm{H}_{\mathrm{A}}\right)$, $1.84\left(\right.$ app t, $\left.{ }^{2} J_{2 B / 2 A}={ }^{3} J_{2 B / 3}=12.5 \mathrm{~Hz}, 1 \mathrm{H}, 2-\mathrm{H}_{B}\right), 1.75-1.69\left(\mathrm{~m}, 1 \mathrm{H}, 11-\mathrm{H}_{\mathrm{A}}\right), 1.69-1.59\left(\mathrm{~m}, 4 \mathrm{H}, 5-\mathrm{H}_{\mathrm{B}}, 9-\mathrm{H}\right.$, $12-\mathrm{H}), 1.56-1.53\left(\mathrm{~m}, 1 \mathrm{H}, 8-\mathrm{H}_{\mathrm{A}}\right), 1.50\left(\mathrm{dd},{ }^{3} J_{6 \mathrm{~A} / 5 \mathrm{~A}}=11.7 \mathrm{~Hz},{ }^{2} J_{6 \mathrm{~A} / 6 \mathrm{~B}}=7.9 \mathrm{~Hz}, 1 \mathrm{H}, 6-\mathrm{H}_{\mathrm{A}}\right), 1.35(\mathrm{dd}$, $\left.{ }^{2} J_{11 \mathrm{~B} / 11 \mathrm{~A}}=13.0 \mathrm{~Hz},{ }^{3} J_{11 \mathrm{~B} / 12 \mathrm{~A}}=8.0 \mathrm{~Hz}, 1 \mathrm{H}, 11-\mathrm{H}_{\mathrm{B}}\right), 1.24-1.22\left(\mathrm{~m}, 1 \mathrm{H}, 8-\mathrm{H}_{\mathrm{B}}\right), 1.21(\mathrm{~s}, 3 \mathrm{H}, 19-\mathrm{H}), 1.19-1.14(\mathrm{~m}$, $\left.1 \mathrm{H}, 6-\mathrm{H}_{\mathrm{B}}\right), 1.09-1.06(\mathrm{~m}, 6 \mathrm{H}, 16-\mathrm{H}, 17-\mathrm{H}, 18-\mathrm{H}), 0.96\left(\mathrm{~d},{ }^{3} \mathrm{~J}=6.7 \mathrm{~Hz}, 3 \mathrm{H}, 16-\mathrm{H}, 17-\mathrm{H}\right) .{ }^{13} \mathrm{C}$ NMR $(200 \mathrm{MHz}$, $\left.\mathrm{C}_{6} \mathrm{D}_{6}\right) \delta 140.42(P h), 128.56(P h), 128.29(P h), 127.34(P h), 95.57(\mathrm{C}-1), 87.21(\mathrm{C}-4), 82.11(\mathrm{C}-14)$, 81.04 (C-13), 80.23 (C-10), $63.03(B n), 53.68$ (C-9), 45.70 (C-3), 42.99 (C-7), 40.14 (C-6), 35.08 (C-8), 34.50 (C-5), 33.07 (C-15), 30.81 (C-2), 26.77 (C-19), 24.90 (C-11), 24.19 (C-12), 19.88 (C-18), 18.46 (C-16, C-17), 18.42 (C-16, C-17), 5.45 (C-20). IR (Diamond-ATR, neat) $\tilde{v}_{\max }: 2956$ (s), $2926(\mathrm{~s}), 2857(\mathrm{~m}), 1729(\mathrm{w})$, $1453(\mathrm{~m}), 1378(\mathrm{~m}), 1348(\mathrm{~m}), 1191(\mathrm{~m}), 1147(\mathrm{~m}), 1022(\mathrm{~m}) \mathrm{cm}^{-1}$. HRMS (EI): calcd for ([M], $\left.\mathrm{C}_{27} \mathrm{H}_{37} \mathrm{O}_{3} \mathrm{l}\right)^{+}$: 536.1787, found: 536.1773 . 


\subsection{Synthesis of (+)-dictyoxetane (1)}

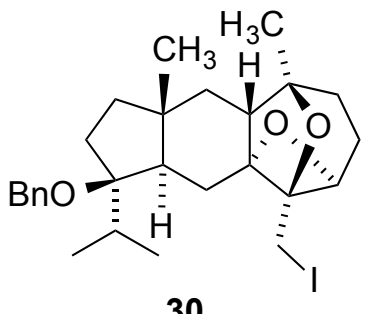

30

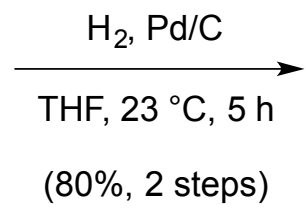

(80\%, 2 steps)

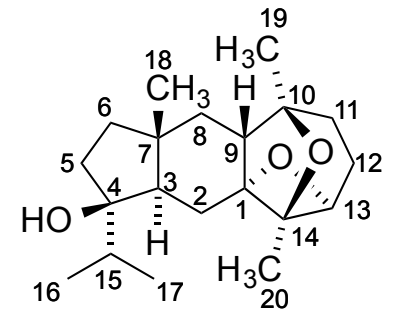

(+)-dictyoxetane (1)

A solution of the crude iodide $\mathbf{3 0}$ (assuming $45.6 \mu \mathrm{mol}, 1$ equiv) in tetrahydrofuran $(5 \mathrm{~mL}$ ) was treated with palladium on carbon (10 wt.\%, $97.1 \mathrm{mg}, 91.2 \mu \mathrm{mol}, 2.00$ equiv) at $23{ }^{\circ} \mathrm{C}$. An atmosphere of hydrogen was maintained by sparging the mixture with a stream of hydrogen gas using a stainless steel needle for $5 \mathrm{~min}$ and vigorous stirring of the suspension was then continued under hydrogen atmosphere at $23{ }^{\circ} \mathrm{C} .{ }^{11}$ After $5 \mathrm{~h}$, the mixture was diluted with ethyl acetate $(20 \mathrm{~mL})$ and filtered through a short pad of Celite. The filtrate was concentrated and the residue was purified by flash-column chromatography on silica gel (30\% ethyl acetate in hexanes) to provide (+)-dictyoxetane (1) (11.7 mg, $80 \%$ over two steps) as a colourless oil.

TLC (40\% ethyl acetate in hexanes): $R_{f}=0.43$ (CAM). ${ }^{1} \mathbf{H}$ NMR (400 MHz, $\left.\mathrm{C}_{6} \mathrm{D}_{6}\right) \delta 4.25$ (br d, $\left.{ }^{3} J_{13 / 12 \mathrm{~A}}=3.9 \mathrm{~Hz}, 1 \mathrm{H}, 13-\mathrm{H}\right), 2.04\left(\mathrm{dd},{ }^{3} J_{3 / 2 \mathrm{~B}}=12.9 \mathrm{~Hz},{ }^{3} J_{3 / 2 \mathrm{~A}}=2.8 \mathrm{~Hz}, 1 \mathrm{H}, 3-\mathrm{H}\right), 1.94-1.86\left(\mathrm{~m}, 1 \mathrm{H}, 5-\mathrm{H}_{\mathrm{A}}\right)$, 1.86-1.67 (m, 4H, 2- $\left.\mathrm{H}_{A}, 5-\mathrm{H}_{B}, 11-\mathrm{H}_{\mathrm{A}}, 12-\mathrm{H}_{\mathrm{A}}\right), 1.67-1.51\left(\mathrm{~m}, 5 \mathrm{H}, 6-\mathrm{H}_{\mathrm{A}}, 8-\mathrm{H}_{\mathrm{A}}, 9-\mathrm{H}, 12-\mathrm{H}_{B}, 15-\mathrm{H}\right)$, 1.48-1.39 (m, 2H, 2- $\left.\mathrm{H}_{\mathrm{B}}, 11-\mathrm{H}_{\mathrm{B}}\right), 1.30(\mathrm{~s}, 3 \mathrm{H}, 20-\mathrm{H}), 1.28(\mathrm{~s}, 3 \mathrm{H}, 19-\mathrm{H}), 1.27-1.24\left(\mathrm{~m}, 1 \mathrm{H}, 8-\mathrm{H}_{\mathrm{B}}\right), 1.12(\mathrm{~s}, 3 \mathrm{H}$, $18-\mathrm{H}), 1.10-1.05\left(\mathrm{~m}, 1 \mathrm{H}, 6-\mathrm{H}_{\mathrm{B}}\right), 0.95\left(\mathrm{~d},{ }^{3} \mathrm{~J}=6.8 \mathrm{~Hz}, 3 \mathrm{H}, 16-\mathrm{H}, 17-\mathrm{H}\right), 0.89\left(\mathrm{~d},{ }^{3} \mathrm{~J}=6.8 \mathrm{~Hz}, 3 \mathrm{H}, 16-\mathrm{H}, 17-\mathrm{H}\right)$, 0.54 (br s, 1H, 4-OH). ${ }^{13} \mathrm{C}$ NMR (100 MHz, $\left.\mathrm{C}_{6} \mathrm{D}_{6}\right) \delta 97.05$ (C-1), 82.45 (C-4), 81.17 (C-13), 80.77 (C-14), 79.78 (C-10), 53.30 (C-9), 47.89 (C-3), 42.67 (C-7), 39.72 (C-6), 36.68 (C-15), 35.43 (C-5), 35.08 (C-8), 28.00 (C-2), 27.06 (C-19), 25.13 (C-11), 24.03 (C-12), 20.05 (C-18), 18.58 (C-16, C-17), 17.68 (C-16, C-17), 16.49 (C-20). IR (Diamond-ATR, neat) $\tilde{v}_{\max }: 3484(\mathrm{br}), 2956(\mathrm{~s}), 2928(\mathrm{~s}), 2862(\mathrm{~m}), 1452(\mathrm{~m}), 1388(\mathrm{~m})$, $1375(\mathrm{~m}), 1292(\mathrm{w}), 1148(\mathrm{~m}), 1067(\mathrm{~m}), 1019(\mathrm{~m}) \mathrm{cm}^{-1}$. HRMS (EI): calcd for ([M], $\left.\mathrm{C}_{20} \mathrm{H}_{32} \mathrm{O}_{3}\right)^{+}: 320.2351$, found: 320.2349. $[\alpha]_{D}^{20}=+29.1^{\circ}\left(\mathrm{c}=1.00, \mathrm{CHCl}_{3}\right)$. (Lit.: $.^{12}[\alpha]_{D}^{20}=+35.0^{\circ}\left(\mathrm{c}=3.00, \mathrm{CHCl}_{3}\right)$.

${ }^{1} \mathbf{H}$ NMR $\left(800 \mathrm{MHz}, \mathrm{CDCl}_{3}\right) \delta 4.38\left(\mathrm{br} \mathrm{d},{ }^{3} J_{13 / 12 \mathrm{~A}}=3.3 \mathrm{~Hz}, 1 \mathrm{H}, 13-\mathrm{H}\right), 2.04\left(\mathrm{ddd},{ }^{2} J_{5 \mathrm{~A} / 5 \mathrm{~B}}=14.5 \mathrm{~Hz}\right.$, $\left.{ }^{3} J_{5 \mathrm{~A} / 6 \mathrm{~B}}=9.6 \mathrm{~Hz},{ }^{3} J_{5 \mathrm{~A} / 6 \mathrm{~A}}=1.1 \mathrm{~Hz}, 1 \mathrm{H}, 5-\mathrm{H}_{\mathrm{A}}\right), 1.86-1.79\left(\mathrm{~m}, 4 \mathrm{H}, 2-\mathrm{H}_{\mathrm{A}}, 3-\mathrm{H}, 11-\mathrm{H}_{\mathrm{A}}, 12-\mathrm{H}_{\mathrm{A}}\right), 1.77-1.71(\mathrm{~m}, 2 \mathrm{H}$, 5- $\left.\mathrm{H}_{B}, 15-\mathrm{H}\right), 1.69-1.59\left(\mathrm{~m}, 3 \mathrm{H}, 6-\mathrm{H}_{\mathrm{A}}, 9-\mathrm{H}, 12-\mathrm{H}_{B}\right), 1.54-1.47\left(\mathrm{~m}, 3 \mathrm{H}, 2-\mathrm{H}_{B}, 8-\mathrm{H}_{\mathrm{A}}, 11-\mathrm{H}_{B}\right), 1.40$ (dd, $\left.{ }^{2} J_{8 \mathrm{~B} / 8 \mathrm{~A}}=11.5 \mathrm{~Hz},{ }^{3} J_{8 \mathrm{~B} / 9}=3.4 \mathrm{~Hz}, 1 \mathrm{H}, 8-\mathrm{H}_{\mathrm{B}}\right), 1.37(\mathrm{~s}, 3 \mathrm{H}, 20-\mathrm{H}), 1.30(\mathrm{~s}, 3 \mathrm{H}, 19-\mathrm{H}), 1.21-1.15\left(\mathrm{~m}, 1 \mathrm{H}, 6-\mathrm{H}_{\mathrm{B}}\right)$, $1.09(\mathrm{~s}, 3 \mathrm{H}, 18-\mathrm{H}), 1.08(\mathrm{br} \mathrm{s}, 1 \mathrm{H}, 4-\mathrm{OH}), 0.97\left(\mathrm{~d},{ }^{3} \mathrm{~J}=6.8 \mathrm{~Hz}, 3 \mathrm{H}, 16-\mathrm{H}, 17-\mathrm{H}\right), 0.90\left(\mathrm{~d},{ }^{3} \mathrm{~J}=6.8 \mathrm{~Hz}, 3 \mathrm{H}, 16-\mathrm{H}\right.$, 17-H). ${ }^{13} \mathrm{C}$ NMR (200 MHz, CDCl $) \delta 97.41$ (C-1), 82.87 (C-4), 81.45 (C-13), 80.81 (C-14), 80.23 (C-10), 52.80 (C-9), 48.14 (C-3), 42.65 (C-7), 39.19 (C-6), 36.73 (C-15), 35.97 (C-5), 34.73 (C-8), 27.78 (C-2), 26.86 (C-19), 24.76 (C-11), 23.51 (C-12), 20.13 (C-18), 18.70 (C-16, C-17), 17.64 (C-16, C-17), 16.30 (C-20). 


\section{$3 \quad{ }^{1} \mathrm{H}$ and ${ }^{13} \mathrm{C}$ NMR comparison of natural ${ }^{12}$ and synthetic dictyoxetane}

\begin{tabular}{|c|c|c|c|}
\hline $\begin{array}{c}{ }^{1} \mathrm{H} \\
\text { Position } \\
\end{array}$ & $\begin{array}{c}\text { Natural } \\
\left(360 \mathrm{MHz}, \mathrm{C}_{6} \mathrm{D}_{6}\right)\end{array}$ & $\begin{array}{c}\text { Synthetic } \\
\left(400 \mathrm{MHz}, \mathrm{C}_{6} \mathrm{D}_{6}\right)\end{array}$ & $\Delta \delta(p p m)$ \\
\hline 3 & $2.03(d d, 13.0,2.5)$ & $2.04(\mathrm{dd}, 12.9,2.6)$ & 0.01 \\
\hline $5 a$ & $1.90(\mathrm{dd}, 14.0,9.5)$ & $1.90(\mathrm{dd}, 14.0,9.5)$ & 0 \\
\hline 13 & $4.25(b r d)$ & $4.25(\mathrm{brd})$ & 0 \\
\hline 16 & $0.95(d, 6.5)$ & $0.95(d, 6.8)$ & 0 \\
\hline 17 & $0.88(d, 6.5)$ & $0.89(d, 6.8)$ & 0.01 \\
\hline 18 & $1.11(\mathrm{~s}, 3 \mathrm{H})$ & $1.12(\mathrm{~s}, 3 \mathrm{H})$ & 0.01 \\
\hline 19 & $1.27(\mathrm{~s}, 3 \mathrm{H})$ & $1.28(\mathrm{~s}, 3 \mathrm{H})$ & 0.01 \\
\hline 20 & $1.29(\mathrm{~s}, 3 \mathrm{H})$ & $1.30(\mathrm{~s}, 3 \mathrm{H})$ & 0.01 \\
\hline
\end{tabular}

Resonances of all other protons occur as overlapping multiplets and are not listed in this table.

\begin{tabular}{|c|c|c|c|}
\hline $\begin{array}{c}{ }^{13} \mathrm{C} \\
\text { Position } \\
\end{array}$ & $\begin{array}{c}\text { Natural } \\
\left(50 \mathrm{MHz} \mathrm{CDCl}_{3}\right)\end{array}$ & $\begin{array}{c}\text { Synthetic } \\
\left(200 \mathrm{MHz}, \mathrm{CDCl}_{3}\right)\end{array}$ & $\Delta \delta(p p m)$ \\
\hline 1 & 97.2 & 97.4 & 0.2 \\
\hline 2 & 27.6 & 27.8 & 0.2 \\
\hline 3 & 48.0 & 48.1 & 0.1 \\
\hline 4 & 82.7 & 82.9 & 0.2 \\
\hline 5 & 35.9 & 36.0 & 0.1 \\
\hline 6 & 39.1 & 39.2 & 0.2 \\
\hline 7 & 42.5 & 42.6 & 0.1 \\
\hline 8 & 34.6 & 34.7 & 0.1 \\
\hline 9 & 52.6 & 52.8 & 0.2 \\
\hline 10 & 80.1 & 80.2 & 0.1 \\
\hline 11 & 24.6 & 24.8 & 0.2 \\
\hline 12 & 23.4 & 23.5 & 0.1 \\
\hline 13 & 81.3 & 81.4 & 0.1 \\
\hline 14 & 80.6 & 80.8 & 0.2 \\
\hline 15 & 36.6 & 36.7 & 0.1 \\
\hline 16 & 17.5 & 17.6 & 0.1 \\
\hline 17 & 18.5 & 18.7 & 0.2 \\
\hline 18 & 20.0 & 20.1 & 0.1 \\
\hline 19 & 26.7 & 26.9 & 0.2 \\
\hline 20 & 16.1 & 16.3 & 0.1 \\
\hline
\end{tabular}




\section{X-Ray Crystallographic Data}

The data collections were performed either on an Oxford Diffraction Xcalibur diffractometer, on a Bruker D8Quest diffractometer or on a Bruker D8Venture at $100 \mathrm{~K}$ or at $173 \mathrm{~K}$ using MoKa-radiation $(\lambda=$ $0.71073 \AA$, graphite monochromator). The CrysAlisPro software (version 1.171.33.41)[S8] was applied for the integration, scaling and multi-scan absorption correction of the data. The structures were solved by direct methods with SIR $97^{13}$ and refined by least-squares methods against $F 2$ with SHELXL-97. ${ }^{14}$ All nonhydrogen atoms were refined anisotropically. The hydrogen atoms were placed in ideal geometry riding on their parent atoms. Further details are summarized in the tables at the different sections.

\subsection{Triol $\mathbf{4 0}$}

CCDC 1473419 contains the supplementary crystallographic data for triol $\mathbf{4 0}$. These data can be obtained free of charge from The Cambridge Crystallographic Data Centre via www.ccdc.cam.ac.uk/data_request/cif.

Table 1. Triol 40

\begin{tabular}{|c|c|}
\hline net formula & $\mathrm{C}_{20} \mathrm{H}_{34} \mathrm{O}_{4}$ \\
\hline$M_{\mathrm{r}} / \mathrm{g} \mathrm{mol}^{-1}$ & 338.47 \\
\hline crystal size/mm & $0.100 \times 0.050 \times 0.040$ \\
\hline$T / \mathrm{K}$ & 100.(2) \\
\hline radiation & MoK $\alpha$ \\
\hline diffractometer & 'Bruker D8 Venture TXS' \\
\hline crystal system & orthorhombic \\
\hline space group & 'P 212121 ' \\
\hline$a / \AA$ & $8.5285(6)$ \\
\hline$b / \AA$ & $14.0549(9)$ \\
\hline$c / \AA$ & $15.1362(10)$ \\
\hline$\alpha /^{\circ}$ & 90 \\
\hline$\beta /^{\circ}$ & 90 \\
\hline$\gamma /{ }^{\circ}$ & 90 \\
\hline$V / \AA^{3}$ & $1814.3(2)$ \\
\hline$Z$ & 4 \\
\hline calc. density/g cm $\mathrm{cm}^{-3}$ & 1.239 \\
\hline$\mu / \mathrm{mm}^{-1}$ & 0.084 \\
\hline absorption correction & Multi-Scan \\
\hline transmission factor range & $0.8239-0.9580$ \\
\hline refls. measured & 7152 \\
\hline$R_{\text {int }}$ & 0.0244 \\
\hline mean $\sigma(I) / I$ & 0.0332 \\
\hline$\theta$ range & $3.101-25.364$ \\
\hline observed refls. & 2889 \\
\hline$x, y$ (weighting scheme) & $0.0354,0.6797$ \\
\hline hydrogen refinement & $\mathrm{C}-\mathrm{H}$ : constr, $\mathrm{O}-\mathrm{H}$ : refall \\
\hline Flack parameter & $-0.6(5)$ \\
\hline refls in refinement & 3234 \\
\hline parameters & 234 \\
\hline restraints & 0 \\
\hline$R\left(F_{\text {obs }}\right)$ & 0.0367 \\
\hline
\end{tabular}




\begin{tabular}{ll}
\hline$R_{\mathrm{w}}\left(F^{2}\right)$ & 0.0916 \\
$S$ & 1.101 \\
shift/error & 0.001 \\
max electron density/e $\AA^{-3}$ & 0.143 \\
min electron density/e $\AA^{-3}$ & -0.212 \\
\hline
\end{tabular}

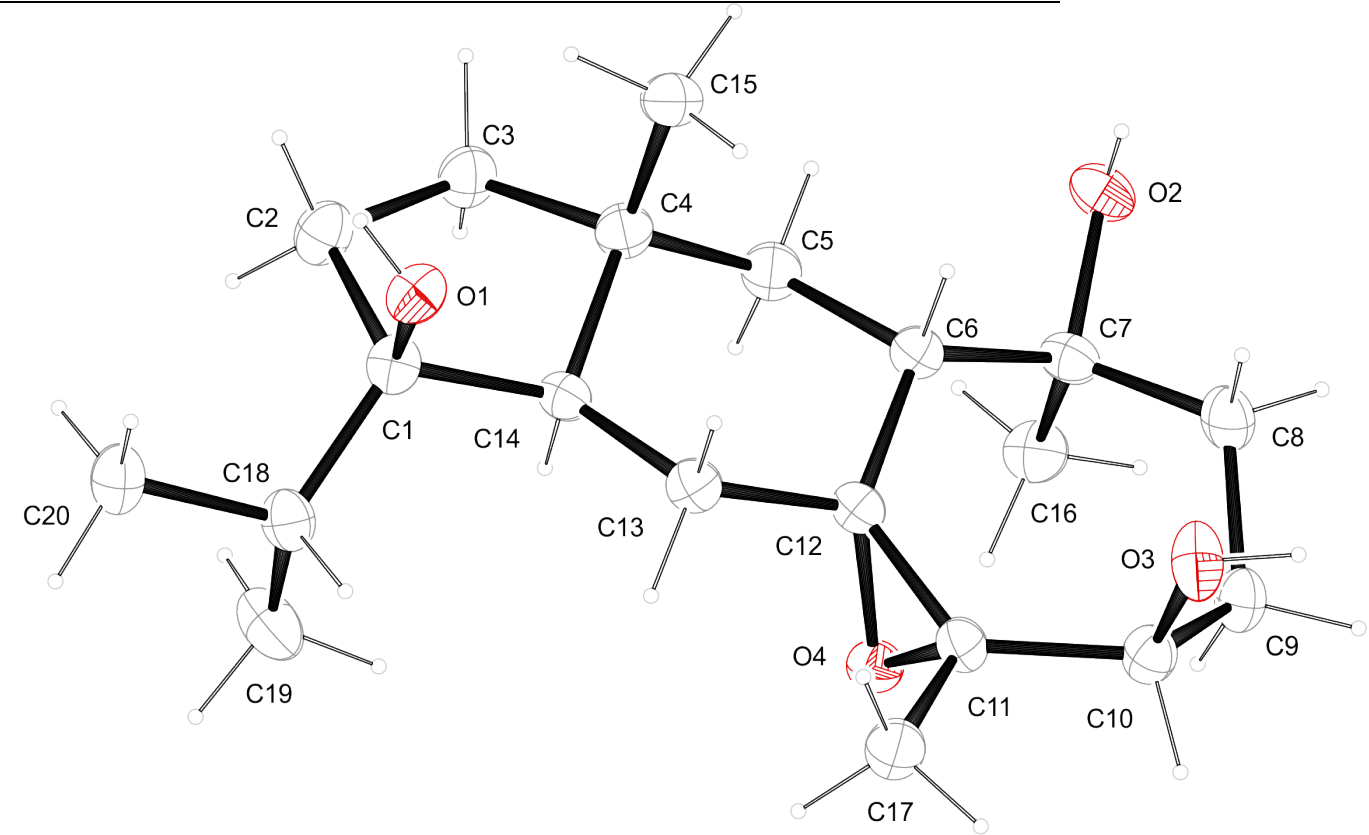

\subsection{1,10,13,14-tetra-epi dictyoxetane (tetra-epi-1)}

CCDC 1473420 contains the supplementary crystallographic data for 1,10,13,14-tetra-epi dictyoxetane (tetra-epi-1). These data can be obtained free of charge from The Cambridge Crystallographic Data Centre via www.ccdc.cam.ac.uk/data_request/cif.

Table 2. 1,10,13,14-tetra-epi dictyoxetane (tetra-epi-1)

\begin{tabular}{ll}
\hline net formula & $\mathrm{C}_{20} \mathrm{H}_{32} \mathrm{O}_{3}$ \\
$M_{\mathrm{r}} / \mathrm{g} \mathrm{mol}^{-1}$ & 320.45 \\
$\mathrm{crystal}$ size/mm & $0.100 \times 0.030 \times 0.020$ \\
$T / \mathrm{K}$ & $100 .(2)$ \\
radiation & MoKa \\
diffractometer & 'Bruker D8 Venture TXS' \\
crystal system & orthorhombic \\
space group & 'P $212121^{\prime}$ \\
$a / \AA$ & $6.3848(4)$ \\
$b / \AA$ & $9.4448(7)$ \\
$c / \AA$ & $28.5710(19)$ \\
$\alpha /{ }^{\circ}$ & 90 \\
$\beta /{ }^{\circ}$ & 90 \\
$\nu /^{\circ}$ & 90 \\
$V / \AA^{3}$ & $1722.9(2)$ \\
\hline
\end{tabular}




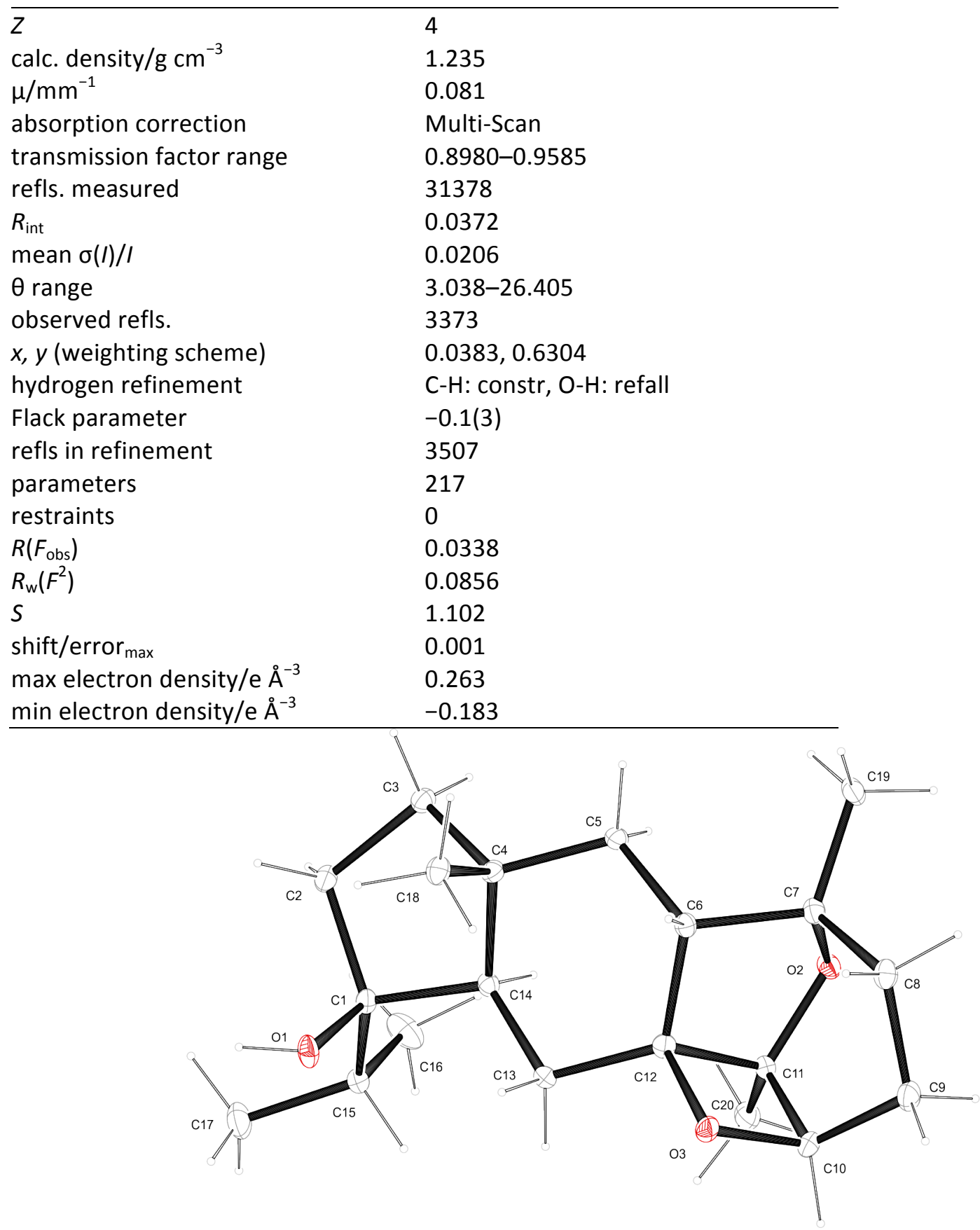

\subsection{Diol 21}

CCDC 1473421 contains the supplementary crystallographic data for diol 21. These data can be obtained free of charge from The Cambridge Crystallographic Data Centre via www.ccdc.cam.ac.uk/data_request/cif.

\section{Table 3. Diol 21}

net formula

$M_{\mathrm{r}} / \mathrm{g} \mathrm{mol}^{-1}$

crystal size/mm
$\mathrm{C}_{20} \mathrm{H}_{33} \mathrm{BrO}_{3}$

401.38

$0.070 \times 0.050 \times 0.030$ 


\begin{tabular}{|c|c|}
\hline$T / \mathrm{K}$ & 100.(2) \\
\hline radiation & MoKa \\
\hline diffractometer & 'Bruker D8 Venture TXS' \\
\hline crystal system & orthorhombic \\
\hline space group & 'P 212121 ' \\
\hline$a / \AA$ & $7.2444(5)$ \\
\hline$b / \AA$ & $10.8801(7)$ \\
\hline$c / \AA$ & $23.8676(15)$ \\
\hline$\alpha /^{\circ}$ & 90 \\
\hline$\beta /^{\circ}$ & 90 \\
\hline$\gamma /{ }^{\circ}$ & 90 \\
\hline$V / \AA^{3}$ & $1881.2(2)$ \\
\hline$Z$ & 4 \\
\hline calc. density/g cm ${ }^{-3}$ & 1.417 \\
\hline$\mu / \mathrm{mm}^{-1}$ & 2.200 \\
\hline absorption correction & Multi-Scan \\
\hline transmission factor range & $0.6527-0.6985$ \\
\hline refls. measured & 33734 \\
\hline$R_{\text {int }}$ & 0.0687 \\
\hline mean $\sigma(I) / I$ & 0.0441 \\
\hline$\theta$ range & $3.172-28.271$ \\
\hline observed refls. & 4453 \\
\hline$x, y$ (weighting scheme) & $0.0293,0.8784$ \\
\hline hydrogen refinement & $\mathrm{C}-\mathrm{H}$ : constr, $\mathrm{O}-\mathrm{H}$ : refall \\
\hline Flack parameter & $0.107(5)$ \\
\hline refls in refinement & 4655 \\
\hline parameters & 230 \\
\hline restraints & 0 \\
\hline$R\left(F_{\mathrm{obs}}\right)$ & 0.0301 \\
\hline$R_{\mathrm{w}}\left(F^{2}\right)$ & 0.0740 \\
\hline$S$ & 1.062 \\
\hline shift/error ${ }_{\max }$ & 0.001 \\
\hline max electron density/e $\AA^{-3}$ & 0.337 \\
\hline min electron density/e $\AA^{-3}$ & -0.499 \\
\hline net formula & $\mathrm{C}_{20} \mathrm{H}_{32} \mathrm{O}_{3}$ \\
\hline$M_{\mathrm{r}} / \mathrm{g} \mathrm{mol}^{-1}$ & 320.45 \\
\hline crystal size/mm & $0.100 \times 0.030 \times 0.020$ \\
\hline$T / \mathrm{K}$ & 100.(2) \\
\hline radiation & MoK $\alpha$ \\
\hline diffractometer & 'Bruker D8 Venture TXS' \\
\hline crystal system & orthorhombic \\
\hline space group & 'P 212121 ' \\
\hline$a / \AA ̊$ & $6.3848(4)$ \\
\hline$b / \AA$ & $9.4448(7)$ \\
\hline$c / \AA$ & $28.5710(19)$ \\
\hline$\alpha /^{\circ}$ & 90 \\
\hline$\beta /^{\circ}$ & 90 \\
\hline$\gamma /{ }^{\circ}$ & 90 \\
\hline$V / \AA^{3}$ & $1722.9(2)$ \\
\hline
\end{tabular}




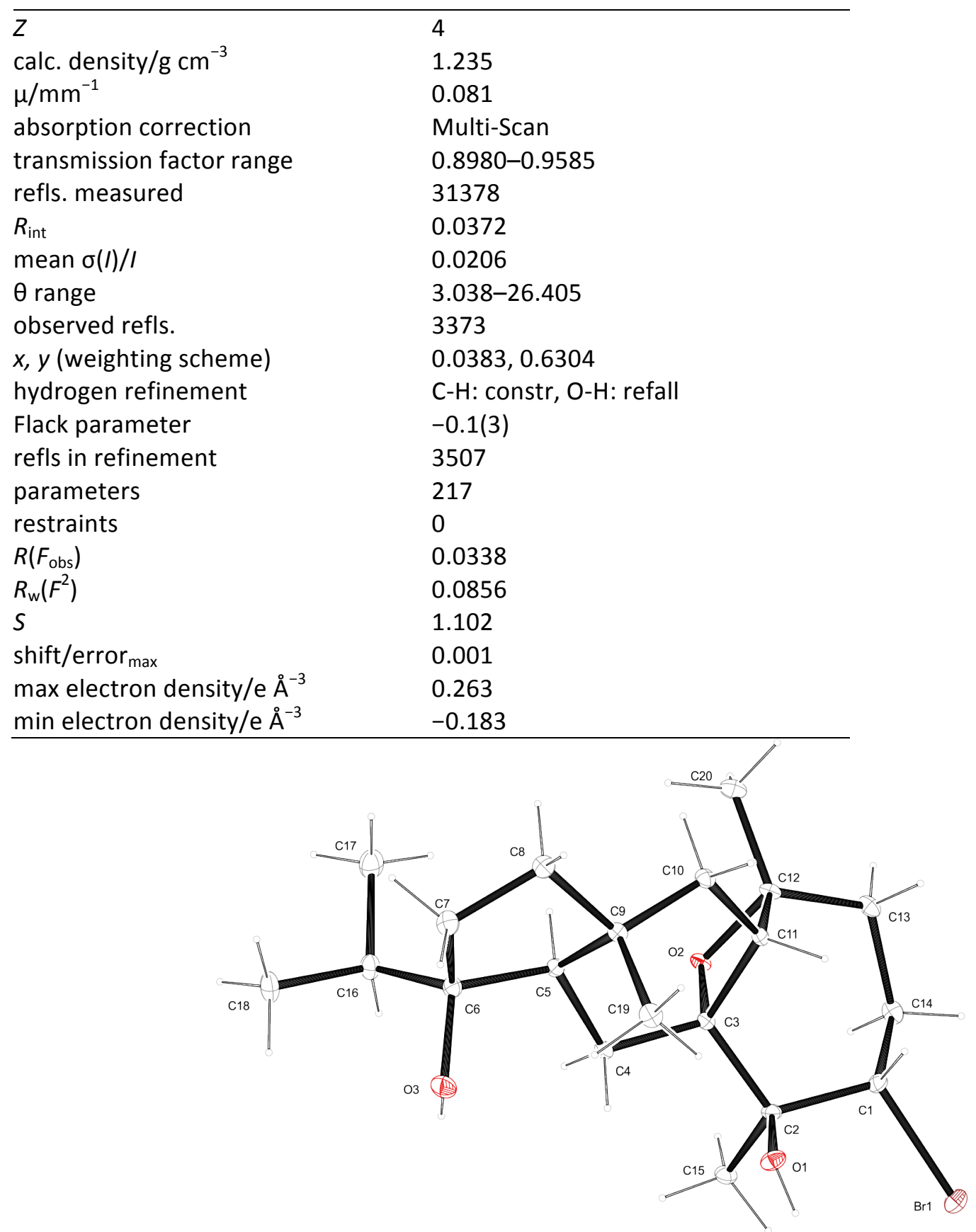




\section{$5 \quad{ }^{1} \mathrm{H}$ and ${ }^{13} \mathrm{C}$ NMR Spectra}




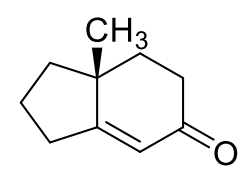

31

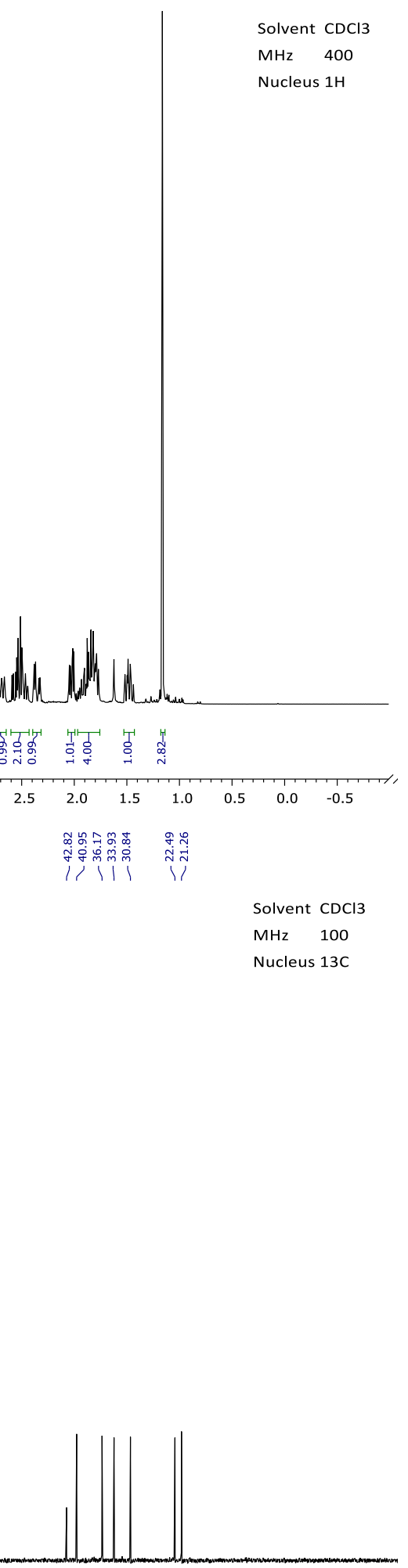

\begin{tabular}{lllllllllllllllllllllllllll}
\hline 210 & 200 & 190 & 180 & 170 & 160 & 150 & 140 & 130 & 120 & 110 & 100 & 90 & 80 & 70 & 60 & 50 & 40 & 30 & 20 & 10 & 0 & -10 &
\end{tabular}


<smiles>C[C@]12CCC=C1CC1(CC2)OCCO1</smiles>

Solvent $\mathrm{CDCl} 3$

$\mathrm{MHz} \quad 400$

32

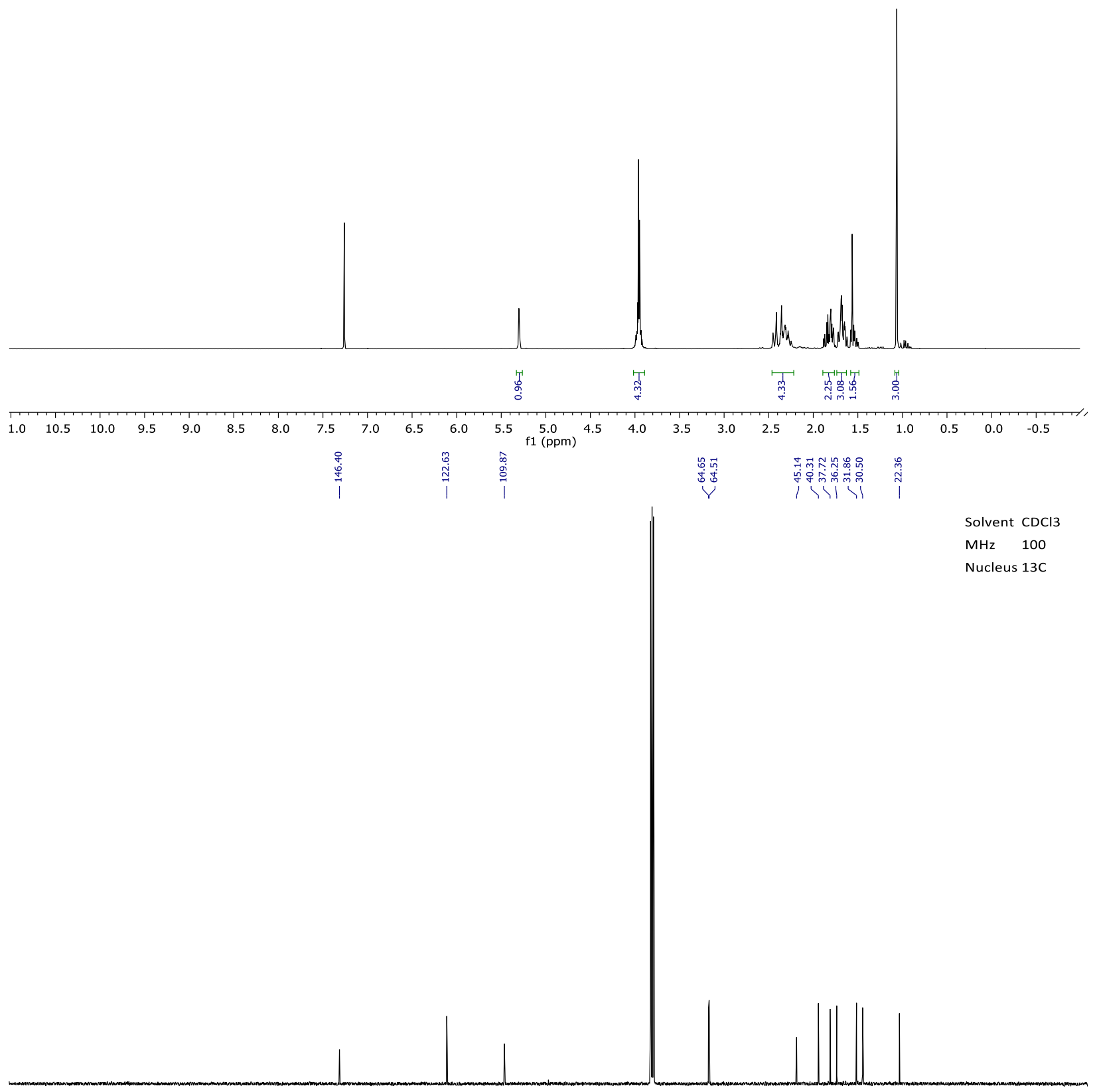

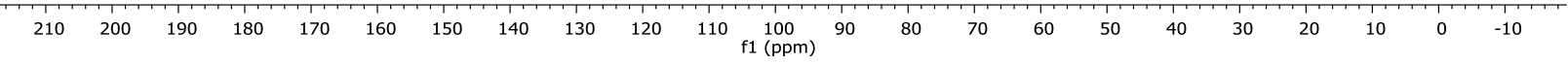




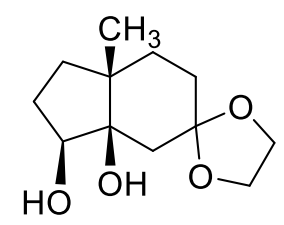

Solvent $\mathrm{CDCl} 3$

$\mathrm{MHz} \quad 400$

33

Nucleus $1 \mathrm{H}$
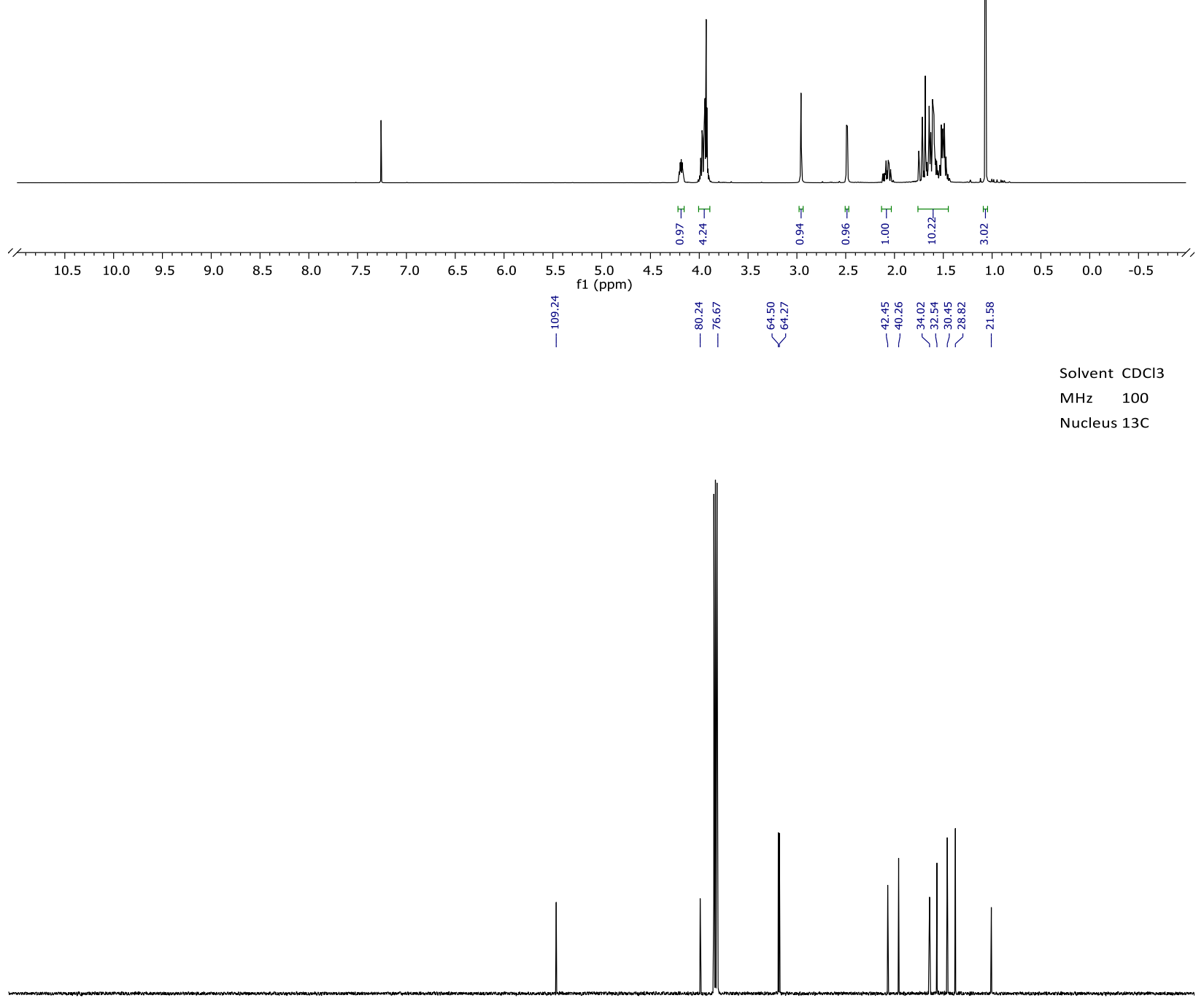

$\begin{array}{lllllllllllllllllllllllllllll}210 & 200 & 190 & 180 & 170 & 160 & 150 & 140 & 130 & 120 & 110 & 100 & 90 & 80 & 70 & 60 & 50 & 40 & 30 & 20 & 10 & 0 & -10 & \end{array}$ 
<smiles>C[C@]12CCC(=O)[C@@H]1CC1(CC2)OCCO1</smiles>

34
Solvent $\mathrm{CDCl} 3$

$\mathrm{MHz} \quad 400$

Nucleus $1 \mathrm{H}$

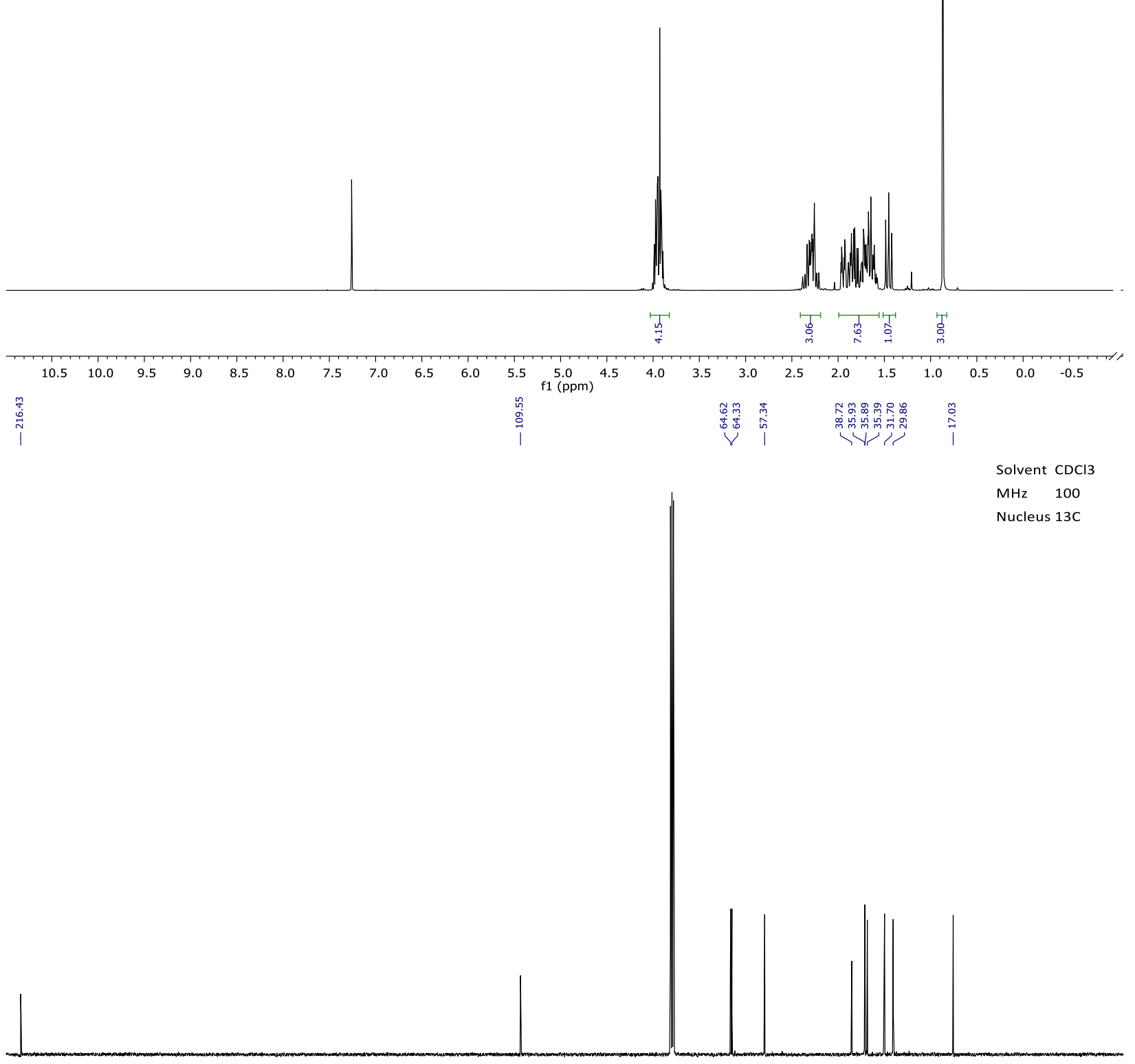

$\begin{array}{lllllllllllllllllllllllllllllllllll}210 & 200 & 190 & 180 & 170 & 160 & 150 & 140 & 130 & 120 & 110 & 100 & 90 & 80 & 70 & 60 & 50 & 40 & 30 & 20 & 10 & 0 & -10 & \end{array}$ 
<smiles>CC(C)[C@]1(O)CC[C@]2(C)CCC3(C[C@H]21)OCCO3</smiles>

Solvent $\mathrm{CDCl} 3$

$\mathrm{MHz} \quad 400$

Nucleus $1 \mathrm{H}$

10

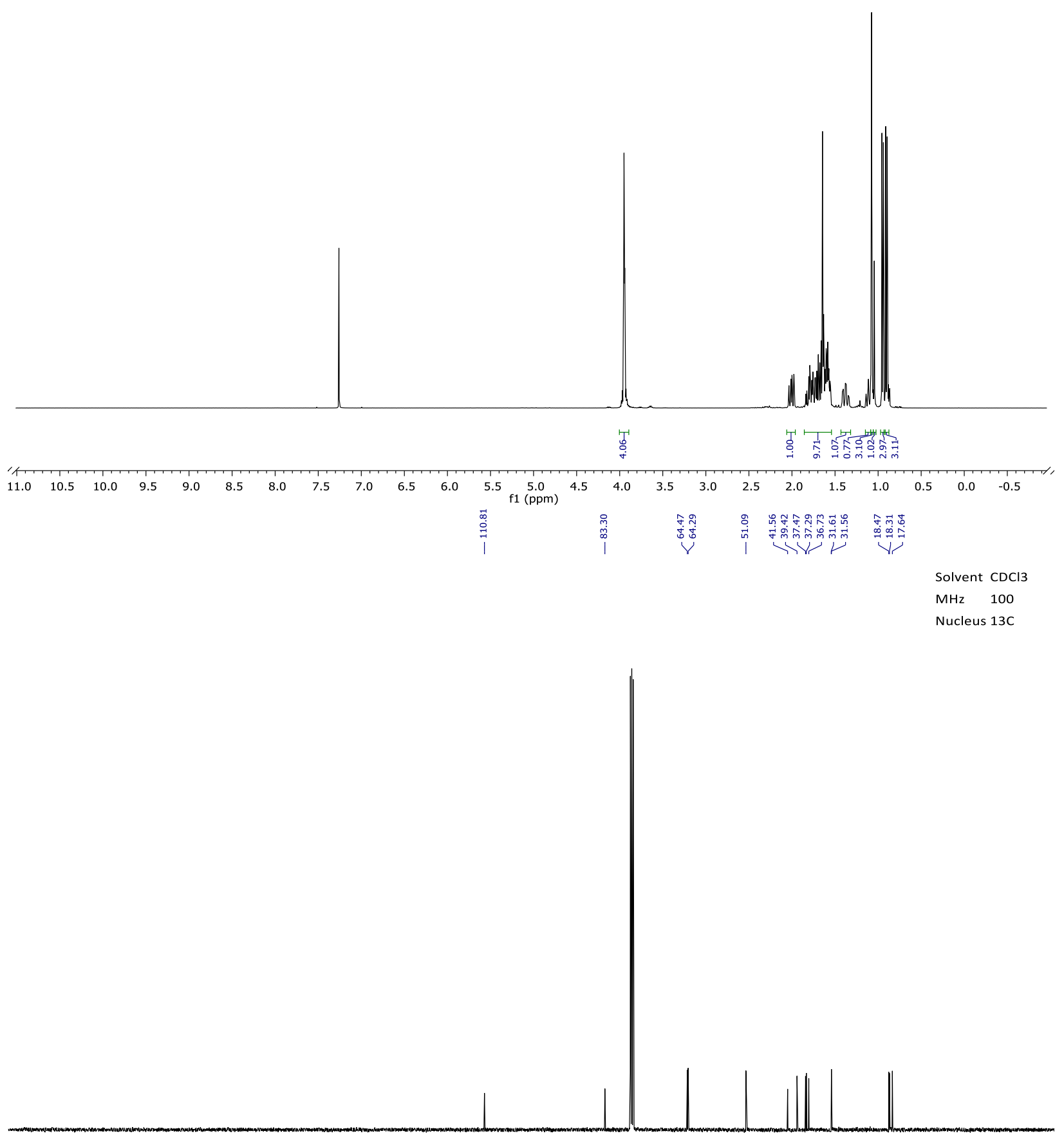

$\begin{array}{lllllllllllllllllllllllllllll}210 & 200 & 190 & 180 & 170 & 160 & 150 & 140 & 130 & 120 & 110 & 100 & 90 & 80 & 70 & 60 & 50 & 40 & 30 & 20 & 10 & 0 & -10 & \end{array}$


<smiles>CC(C)[C@]1(OCc2ccccc2)CC[C@]2(C)CCC3(C[C@@H]21)OCCO3</smiles>

Solvent $\mathrm{CDCl} 3$

$\mathrm{MHz} \quad 400$

Nucleus $1 \mathrm{H}$

35

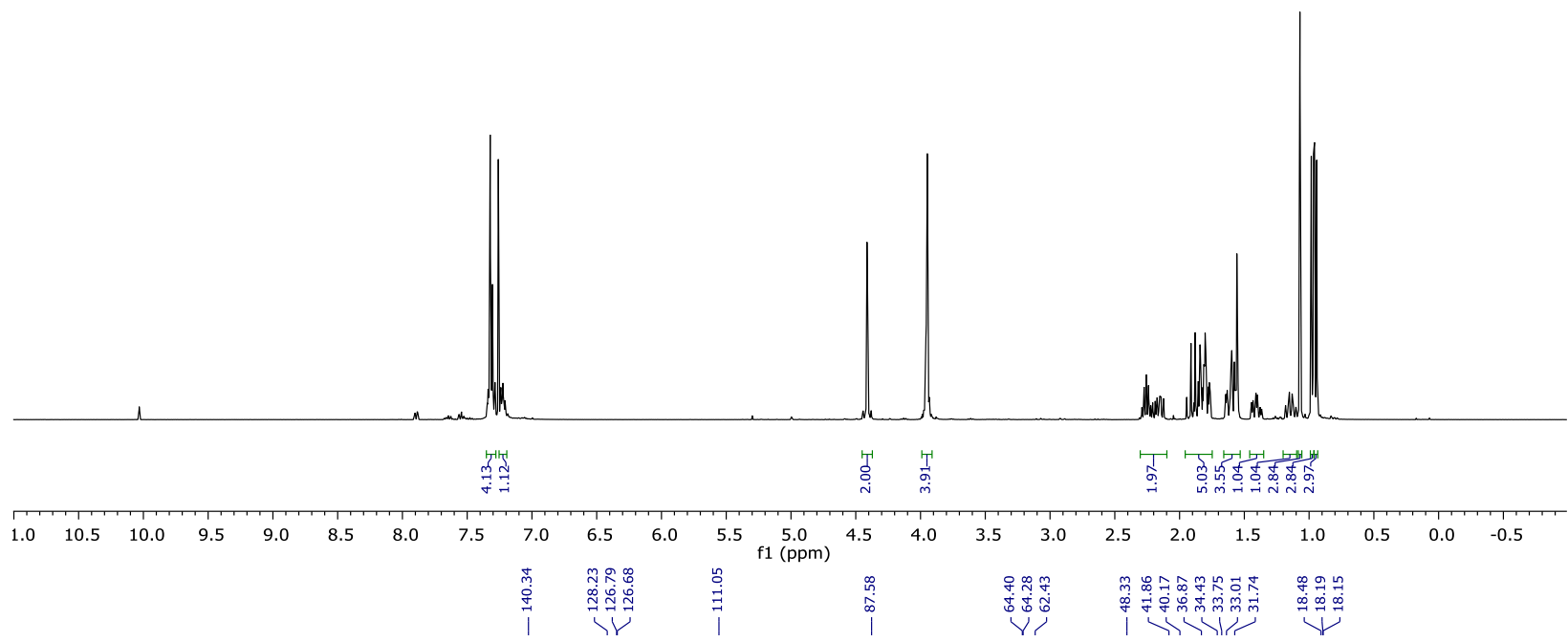

Solvent $\mathrm{CDCl} 3$

$\mathrm{MHz} \quad 100$

Nucleus $13 \mathrm{C}$

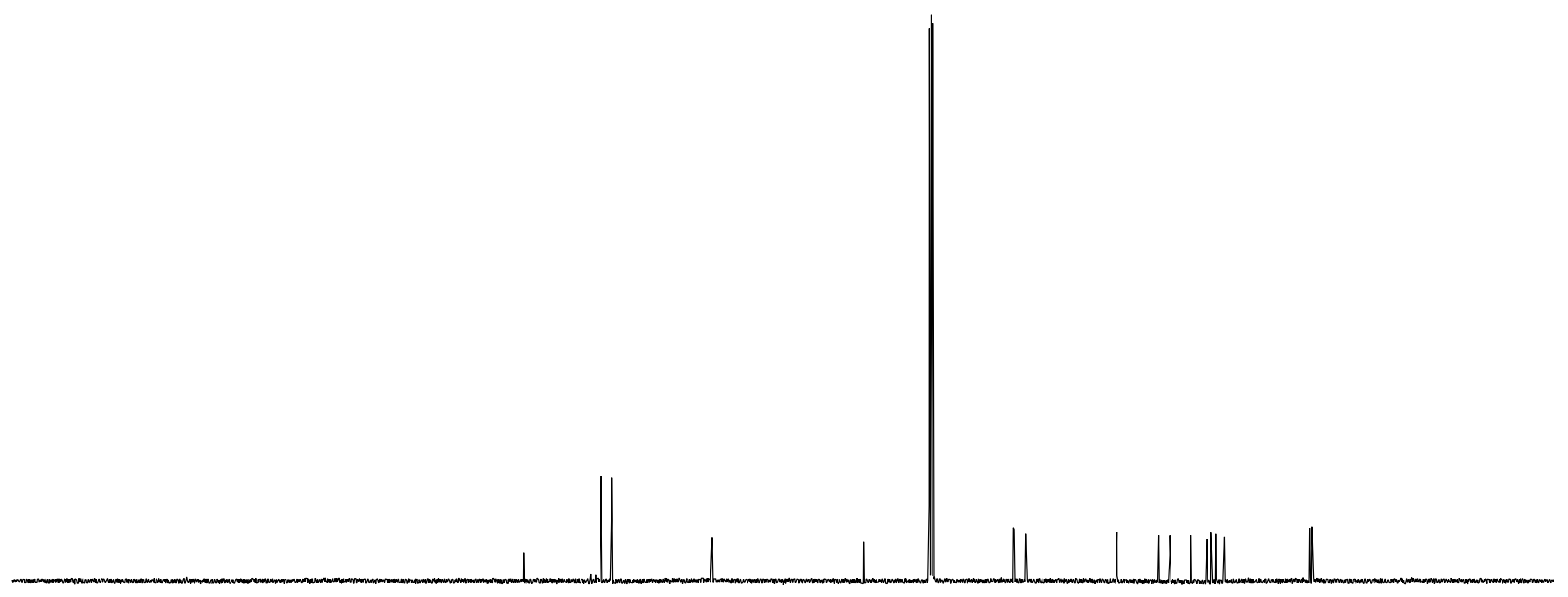


<smiles>CC(C)[C@]1(OCc2ccccc2)CC[C@]2(C)CCC(=O)C[C@@H]21</smiles>

11
Solvent $\mathrm{CDCl} 3$

$\mathrm{MHz} \quad 600$

Nucleus $1 \mathrm{H}$
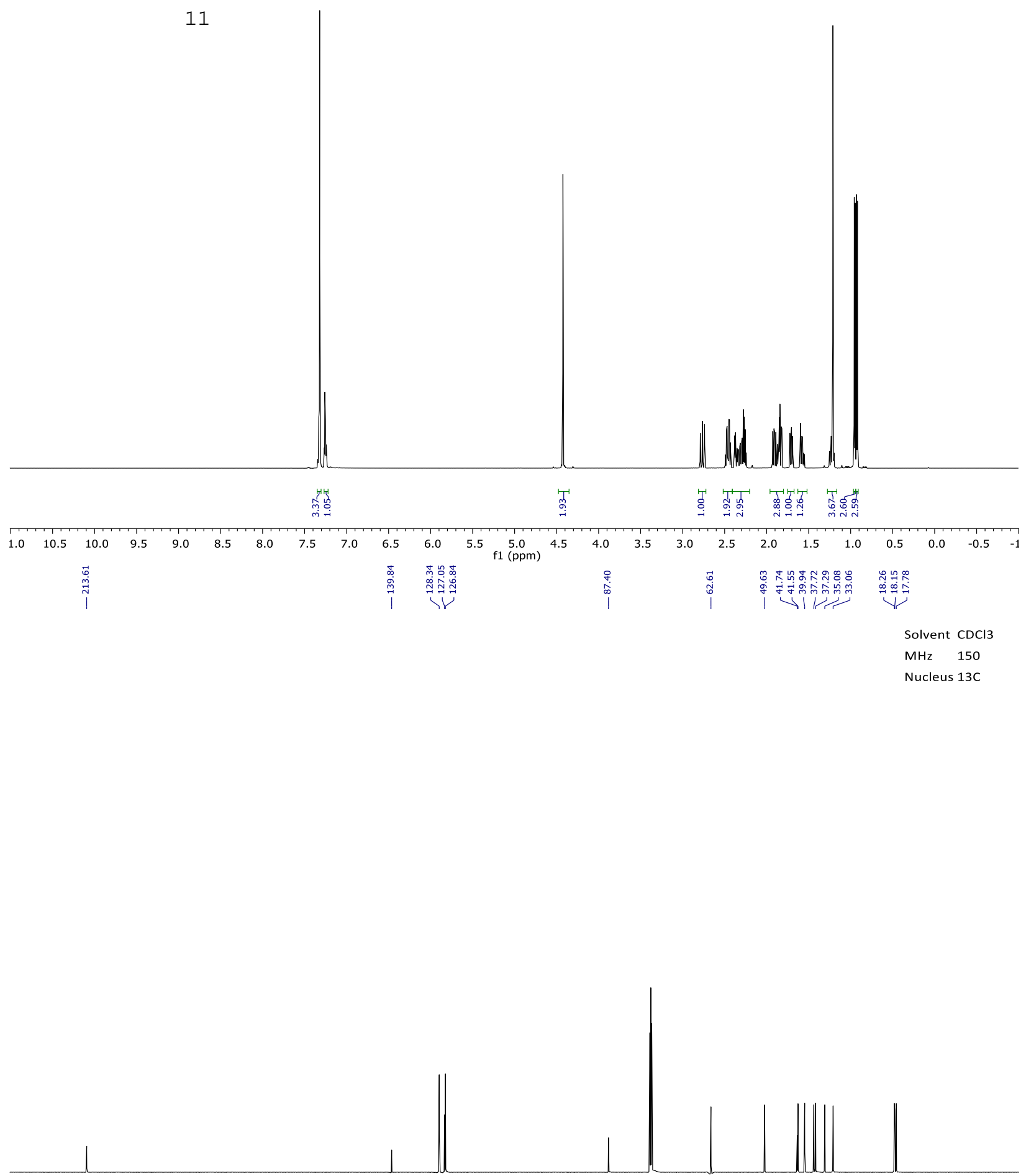

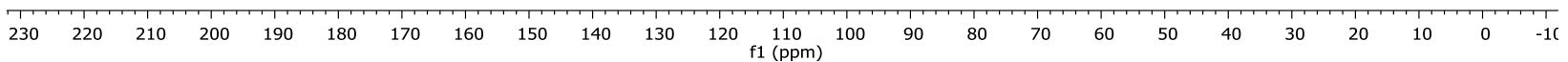


<smiles>CC(C)[C@]1(OCc2ccccc2)CC[C@]2(C)C[C@H]([C@H](C)O[Si](C)(C)C(C)(C)C)C(=O)C[C@@]21C</smiles>

Solvent C6D6

$\mathrm{MHz} \quad 800$

Nucleus $1 \mathrm{H}$

36
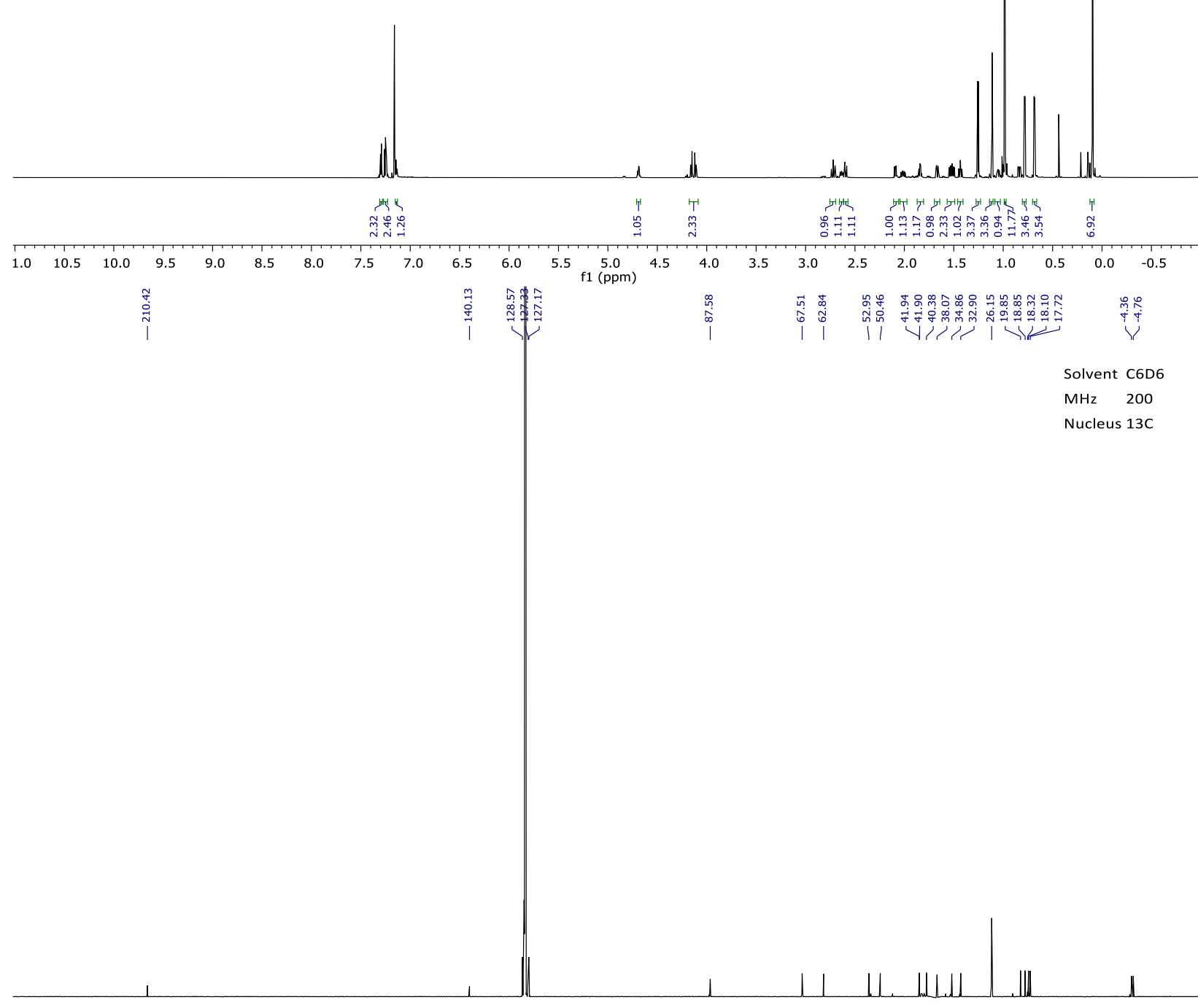

$\begin{array}{llllllllllllllllllllllllllllllllll}230 & 220 & 210 & 200 & 190 & 180 & 170 & 160 & 150 & 140 & 130 & 120 & 110 & 100 & 90 & 80 & 70 & 60 & 50 & 40 & 30 & 20 & 10 & 0 & -10\end{array}$




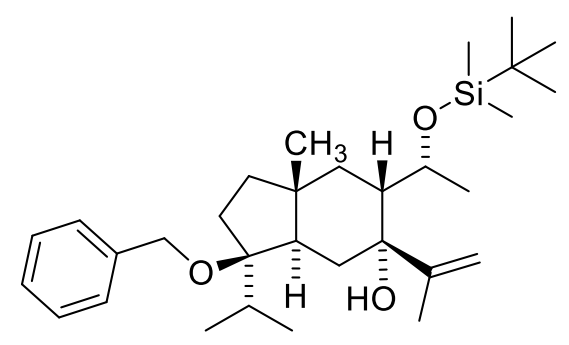

Solvent C6D6

$\mathrm{MHz} \quad 400$

Nucleus $1 \mathrm{H}$

12
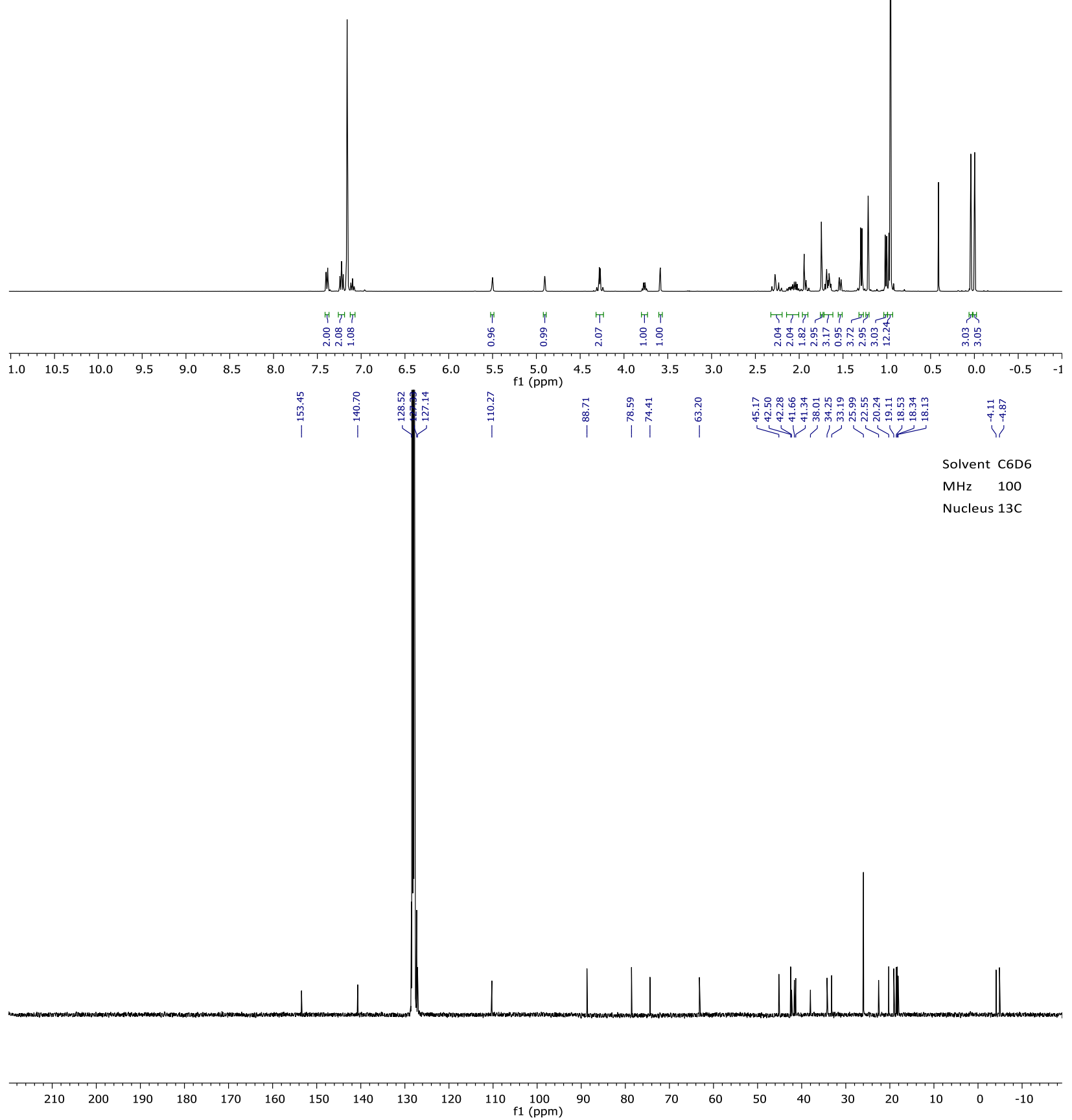


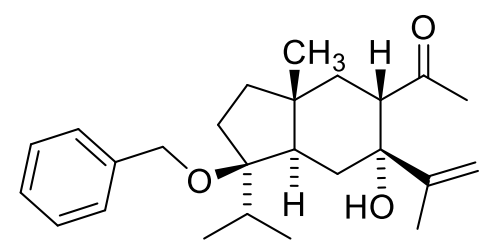

13

Solvent C6D6

$\mathrm{MHz} \quad 400$

Nucleus $1 \mathrm{H}$

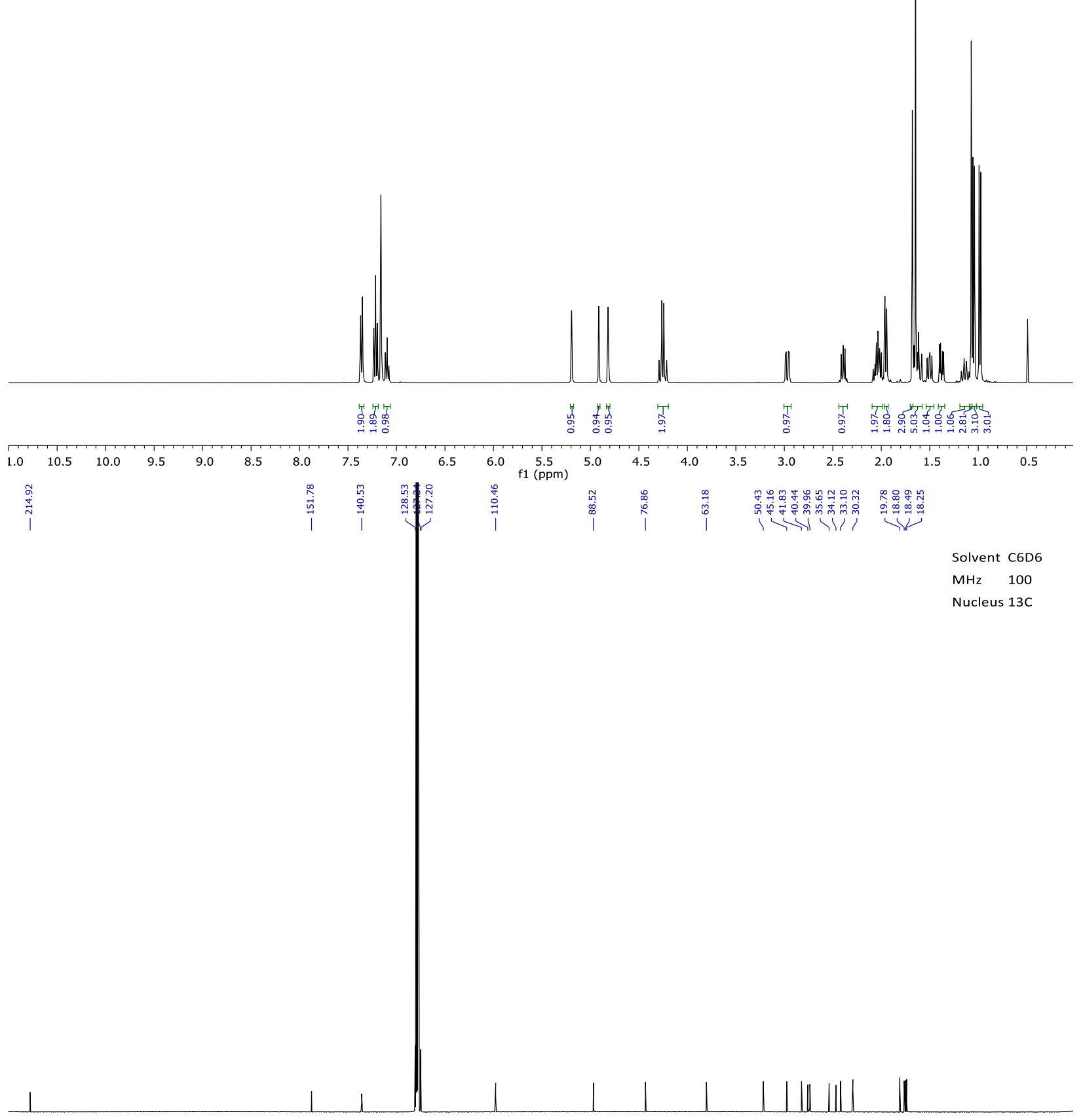

$\begin{array}{llllllllllllllllllllllll}210 & 200 & 190 & 180 & 170 & 160 & 150 & 140 & 130 & 120 & 110 & 100 & 90 & 80 & 70 & 60 & 50 & 40 & 30 & 20 & 10 & 0 & -10\end{array}$




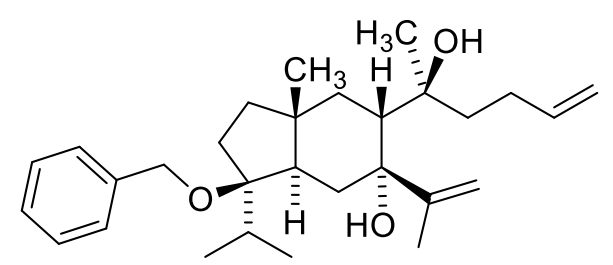

Solvent C6D6

$\mathrm{MHz} \quad 400$

Nucleus $1 \mathrm{H}$

37
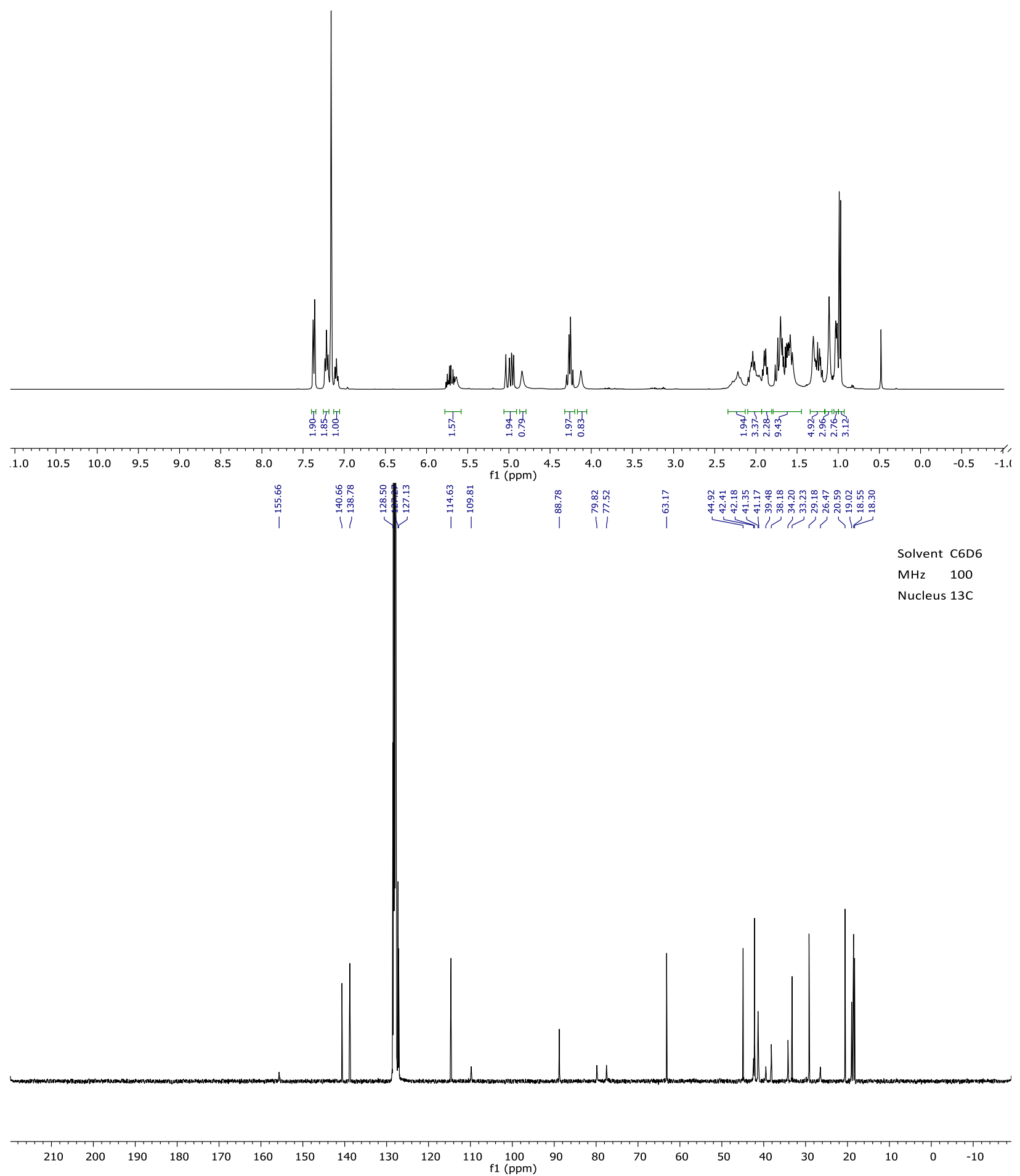


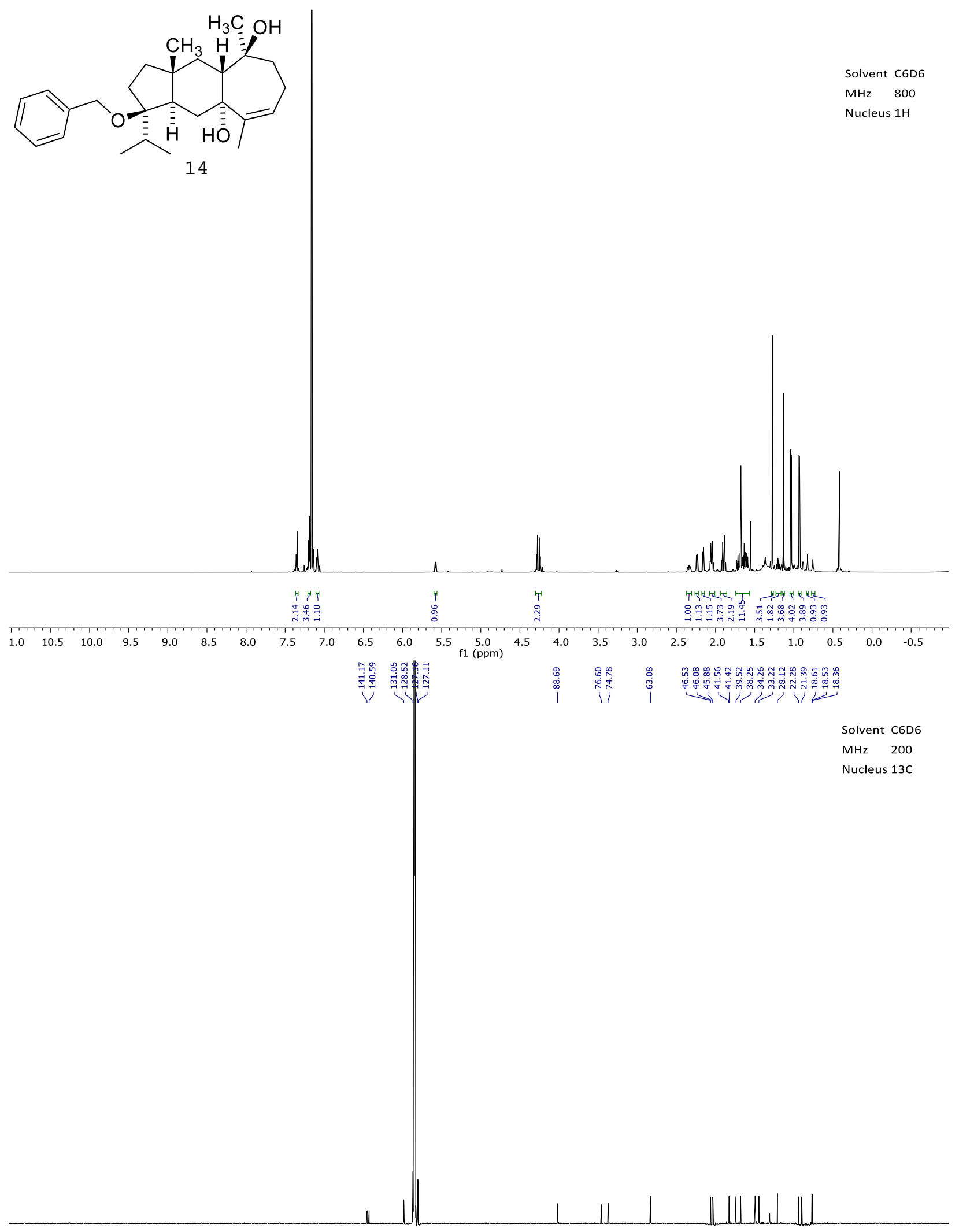

$\begin{array}{lllllllllllllllllllllllllllll}230 & 220 & 210 & 200 & 190 & 180 & 170 & 160 & 150 & 140 & 130 & 120 & 110 & 100 & 90 & 80 & 70 & 60 & 50 & 40 & 30 & 20 & 10 & 0 & -10\end{array}$




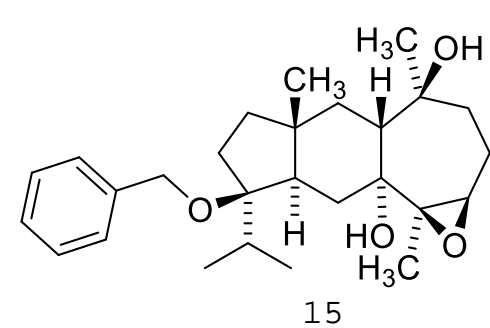

Solvent C6D6 $\mathrm{MHz} \quad 400$ Nucleus $1 \mathrm{H}$ 15
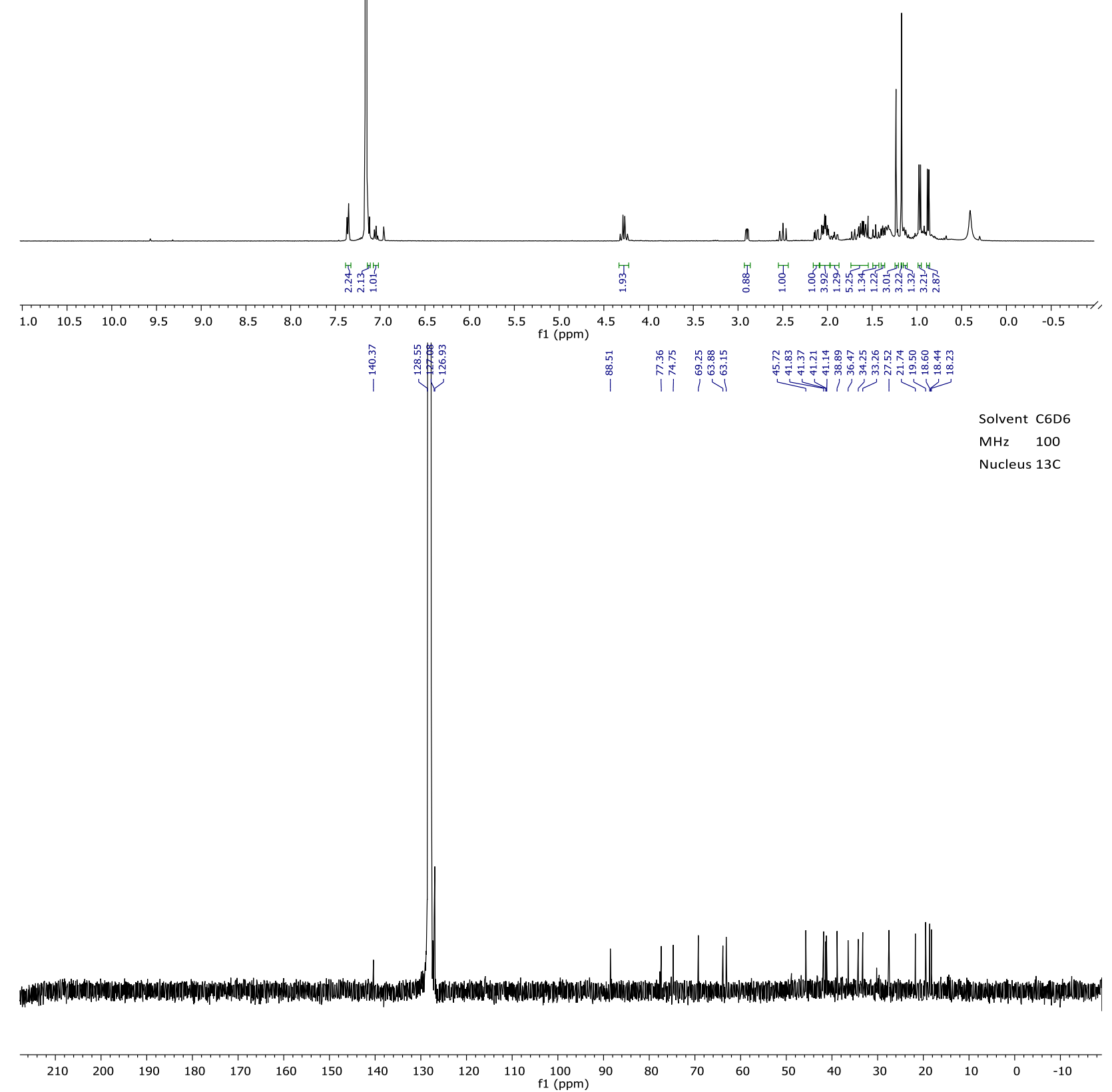


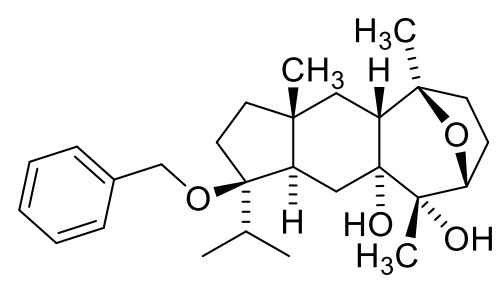

Solvent C6D6

$\mathrm{MHz} \quad 800$

Nucleus $1 \mathrm{H}$ 39

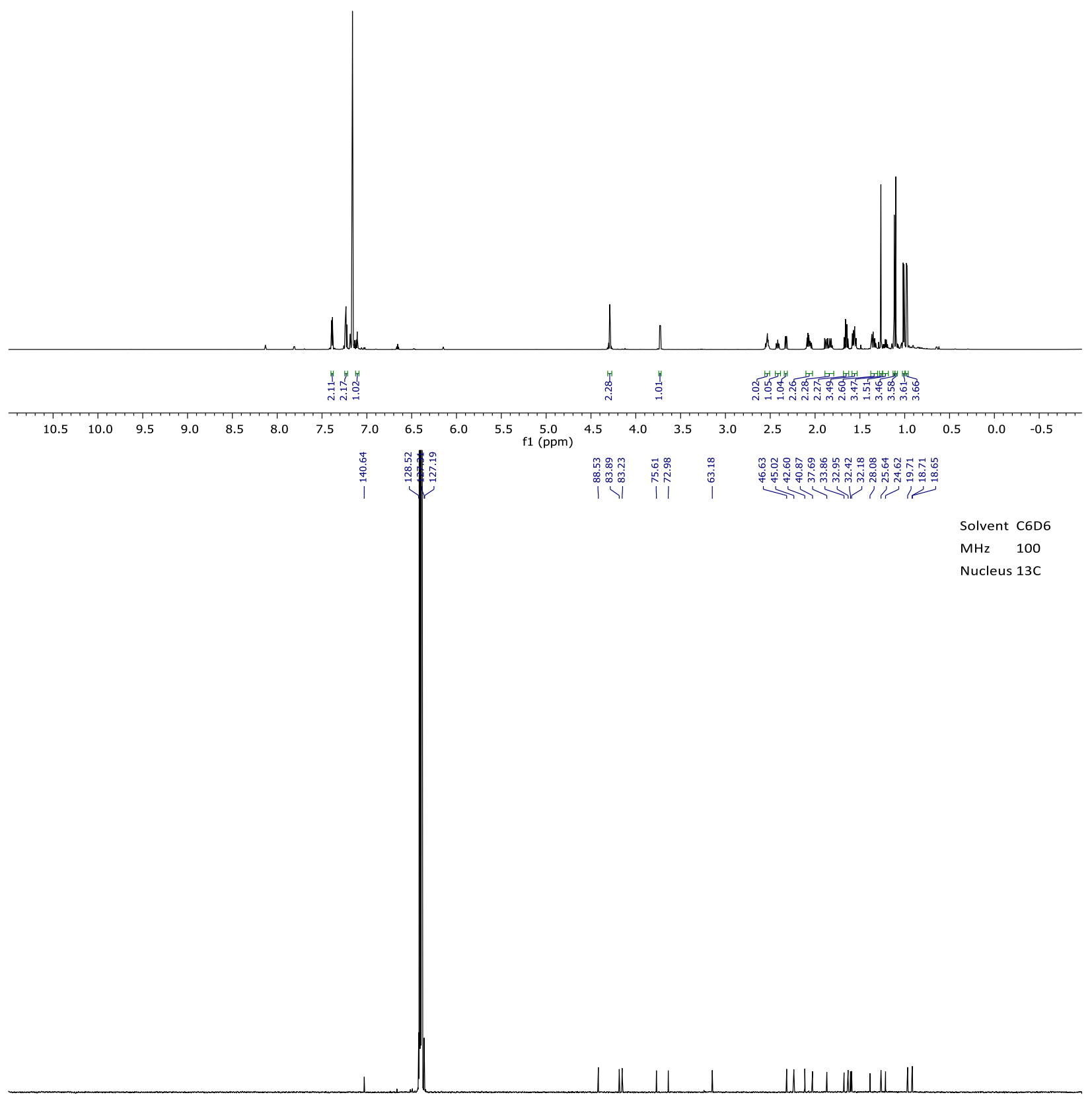

$\begin{array}{lllllllllllllllllllllllllll}210 & 200 & 190 & 180 & 170 & 160 & 150 & 140 & 130 & 120 & 110 & 100 & 90 & 80 & 70 & 60 & 50 & 40 & 30 & 20 & 10 & 0 & -10 & \end{array}$




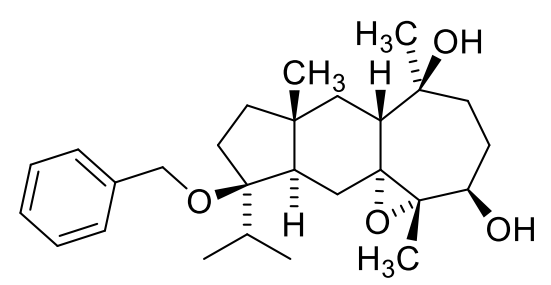

Solvent C6D6

$\mathrm{MHz} \quad 400$

Nucleus $1 \mathrm{H}$

16

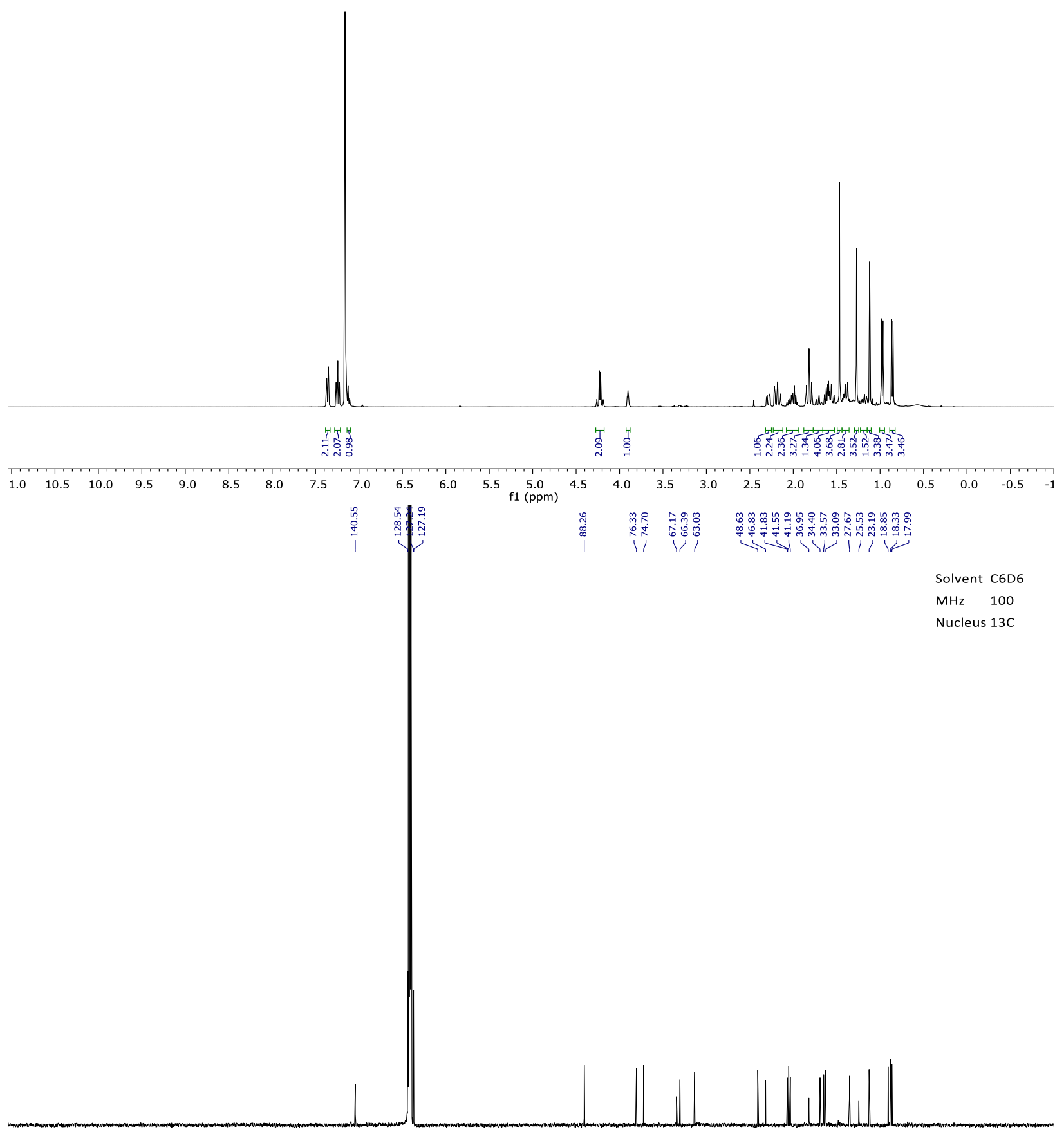

$\begin{array}{lllllllllllllllllllllll}210 & 200 & 190 & 180 & 170 & 160 & 150 & 140 & 130 & 120 & 110 & \begin{array}{l}100 \\ \mathrm{f} 1(\mathrm{ppm})\end{array} & 90 & 80 & 60 & 50 & 40 & 30 & 20 & 10 & 0 & -10 & \end{array}$


<smiles>CC(C)[C@]1(O)CC[C@]2(C)C[C@H]3[C@](C)(O)CC[C@@H](O)[C@@](C)(O)[C@]3(C)C[C@@H]21</smiles>

Solvent $\mathrm{CD} 2 \mathrm{Cl} 2$

$\mathrm{MHz} \quad 800$

Nucleus $1 \mathrm{H}$

40

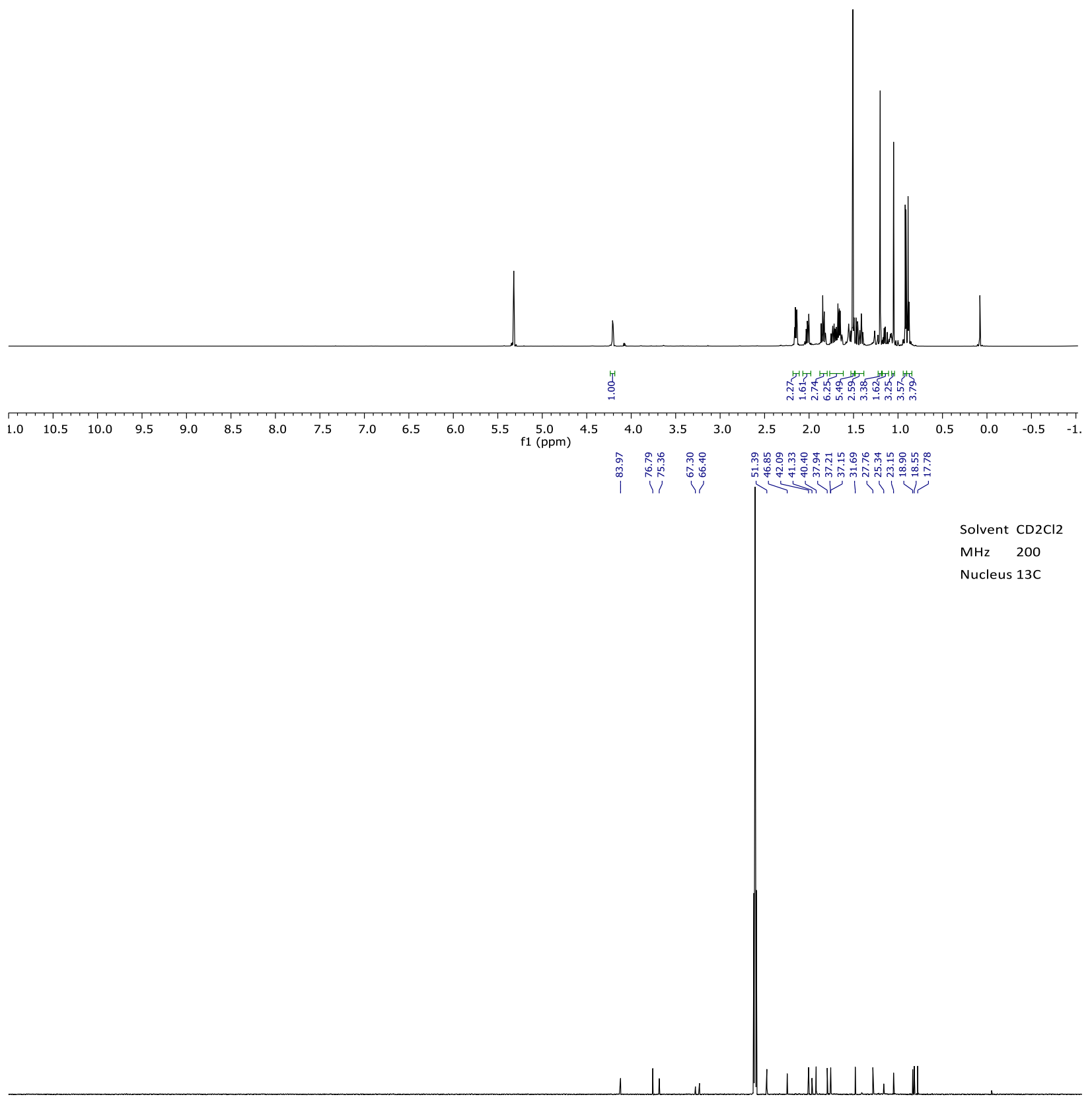

\begin{tabular}{lllllllllllllllllllllllllllllll}
\hline 20 & 210 & 200 & 190 & 180 & 170 & 160 & 150 & 140 & 130 & 120 & 110 & 100 & 90 & 80 & 70 & 60 & 50 & 40 & 30 & 20 & 10 & 0 & -10
\end{tabular} 


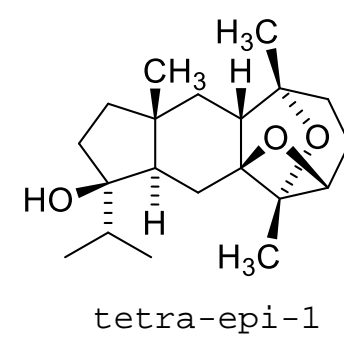

Solvent C6D6

$\mathrm{MHz} \quad 800$

Nucleus $1 \mathrm{H}$

tetra-epi-1
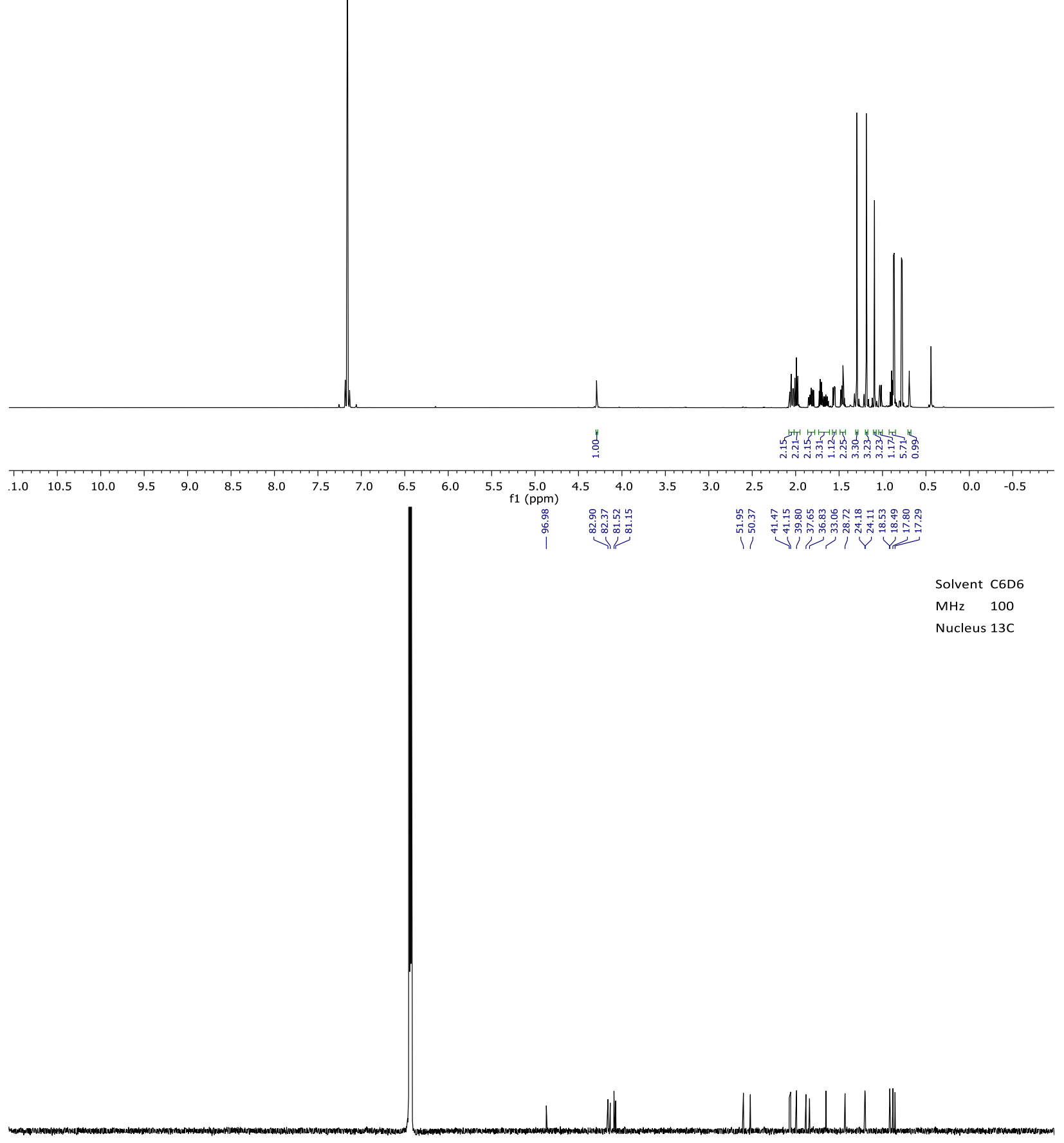

$\begin{array}{lllllllllllllllllllllllll}210 & 200 & 190 & 180 & 170 & 160 & 150 & 140 & 130 & 120 & 110 & 100 & 90 & 80 & 70 & 60 & 50 & 40 & 30 & 20 & 10 & 0 & -10\end{array}$



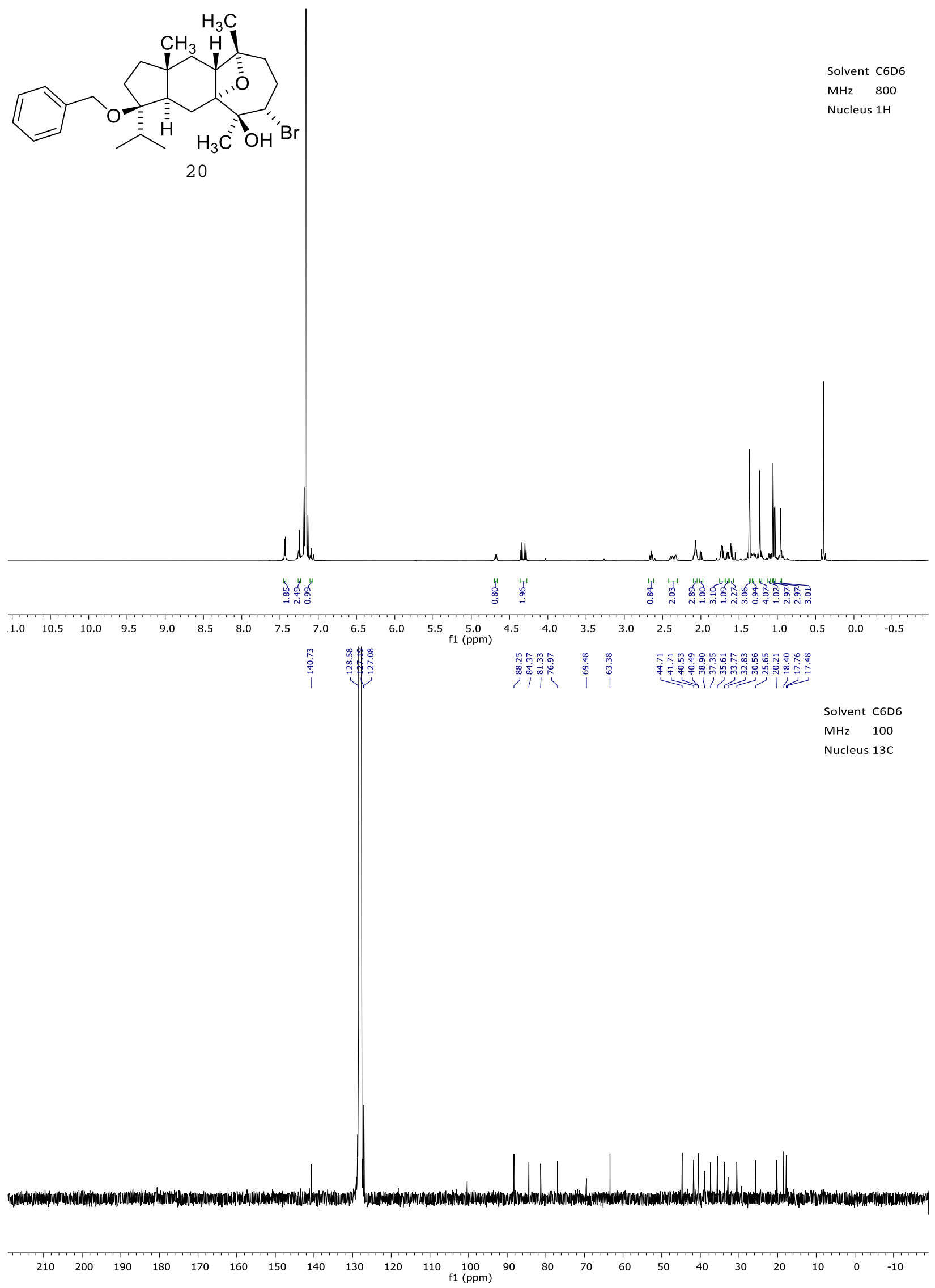


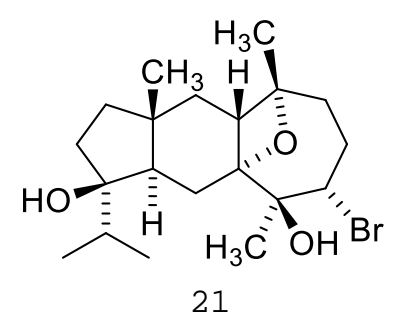

Solvent C6D6 $\mathrm{MHz} \quad 400$

Nucleus $1 \mathrm{H}$ 21

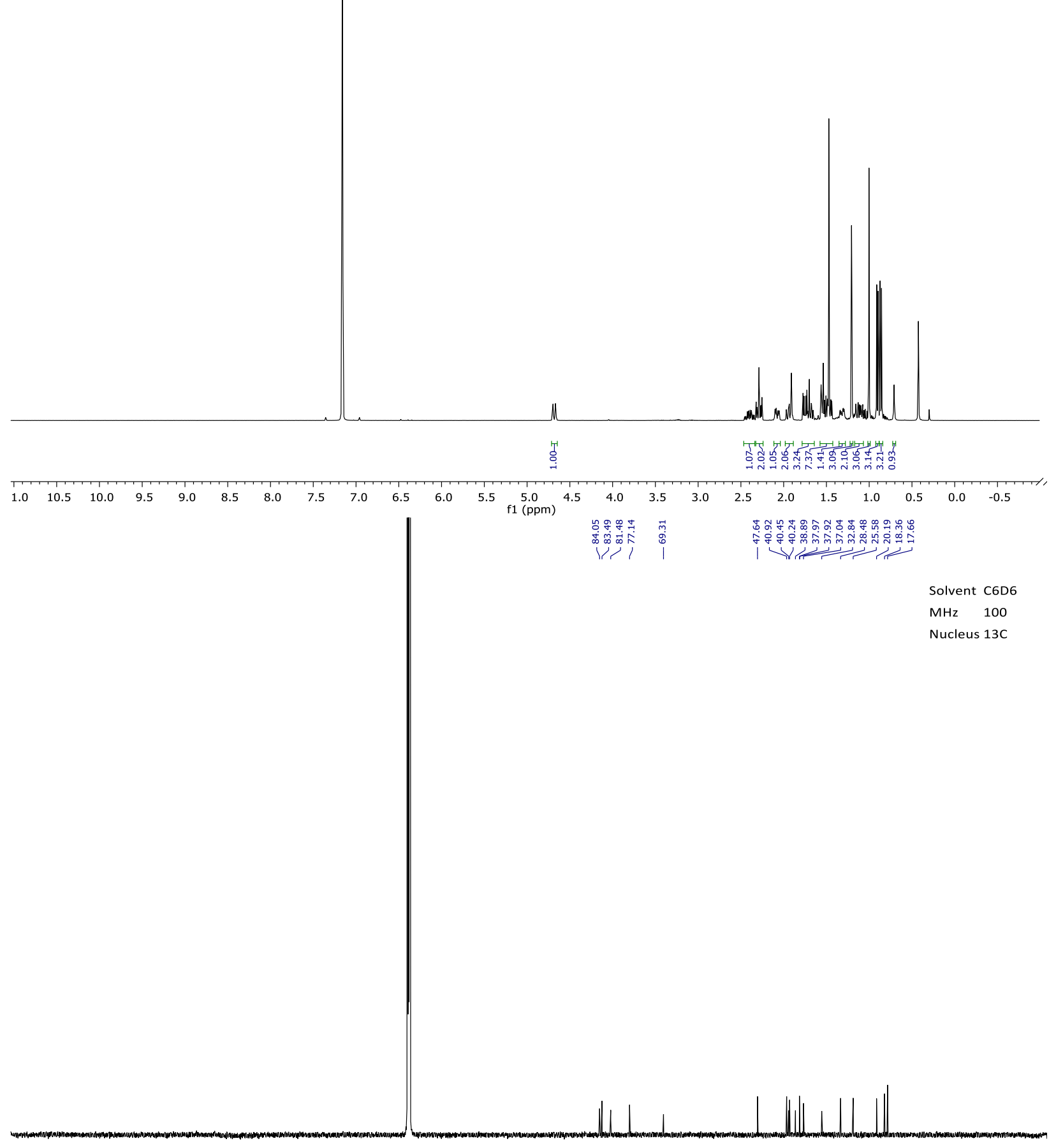

$\begin{array}{llllllllllllllllllllllll}210 & 200 & 190 & 180 & 170 & 160 & 150 & 140 & 130 & 120 & 110 & 100 & 90 & 80 & 70 & 60 & 50 & 40 & 30 & 20 & 10 & 0 & -10 & \end{array}$




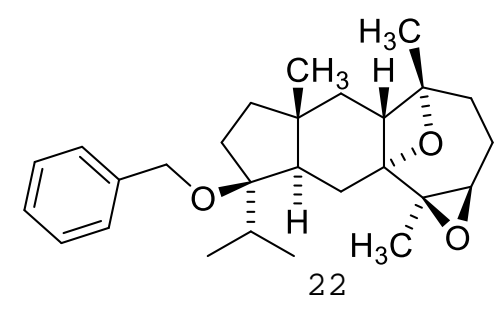

Solvent C6D6 $\mathrm{MHz} \quad 400$

Nucleus $1 \mathrm{H}$ 22

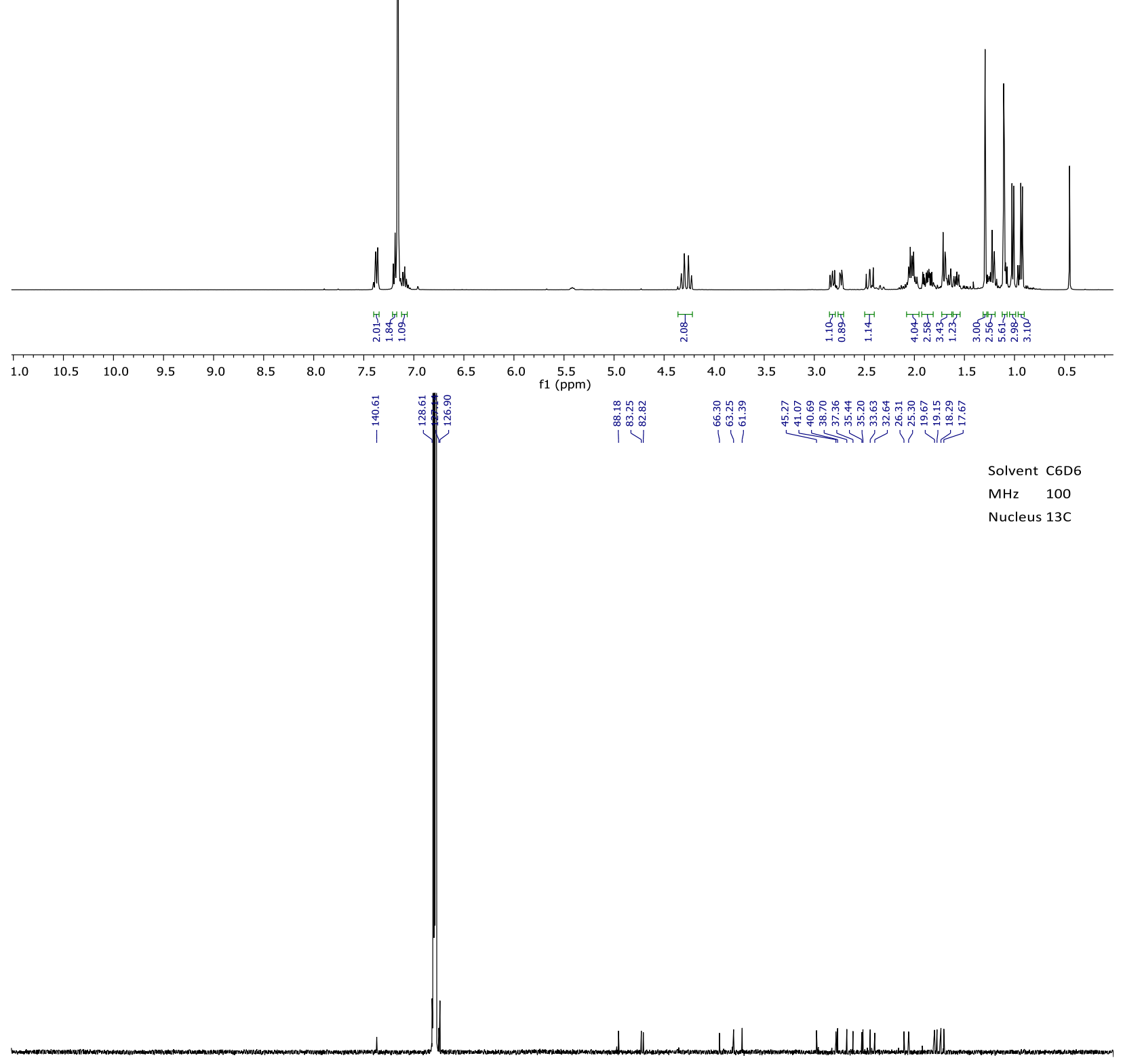

$\begin{array}{lllllllllllllllllllllllll}210 & 200 & 190 & 180 & 170 & 160 & 150 & 140 & 130 & 120 & 110 & 100 & 90 & 80 & 70 & 60 & 50 & 40 & 30 & 20 & 10 & 0 & -10\end{array}$




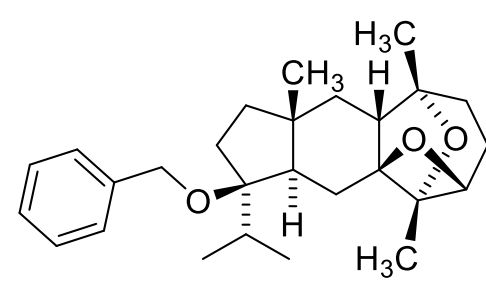

Solvent C6D6 $\mathrm{MHz} \quad 400$ Nucleus $1 \mathrm{H}$ 17

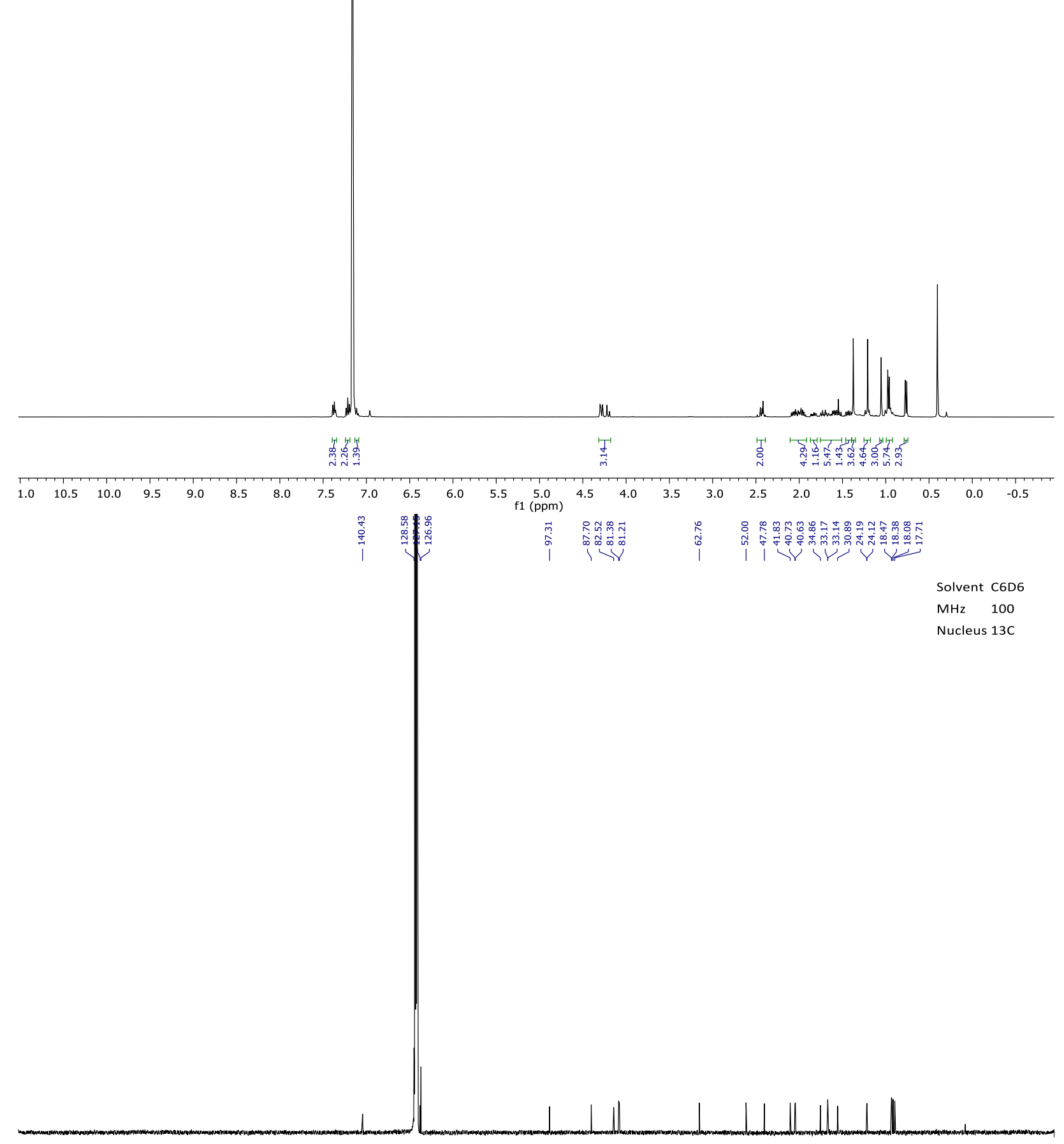

$\begin{array}{llllllllllllllllllllllllll}210 & 200 & 190 & 180 & 170 & 160 & 150 & 140 & 130 & 120 & 110 & 100 & 90 & 80 & 70 & 60 & 50 & 40 & 30 & 20 & 10 & 0 & -10 & \end{array}$




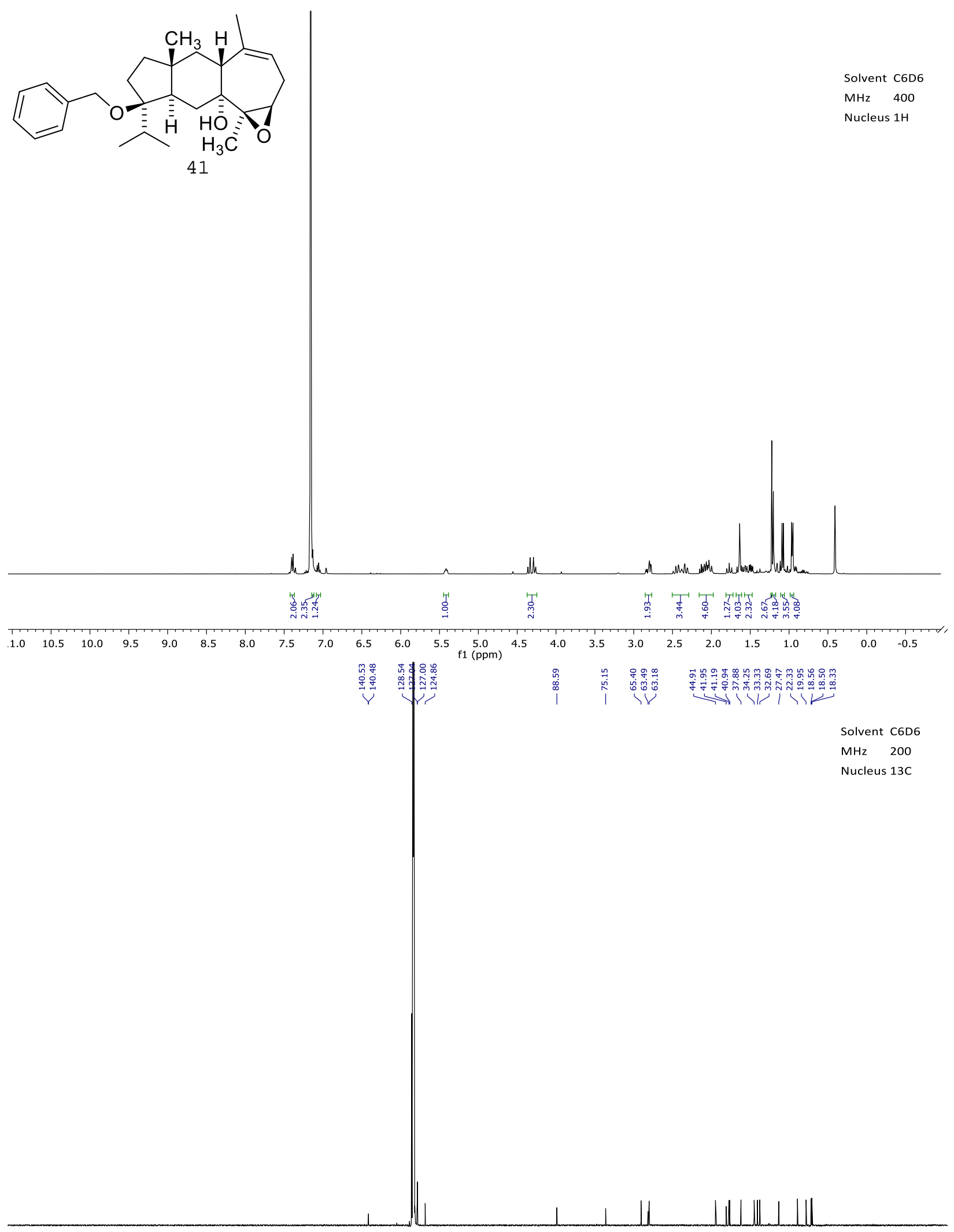

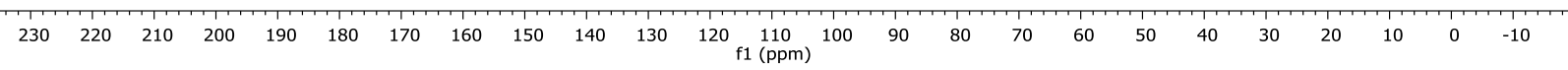




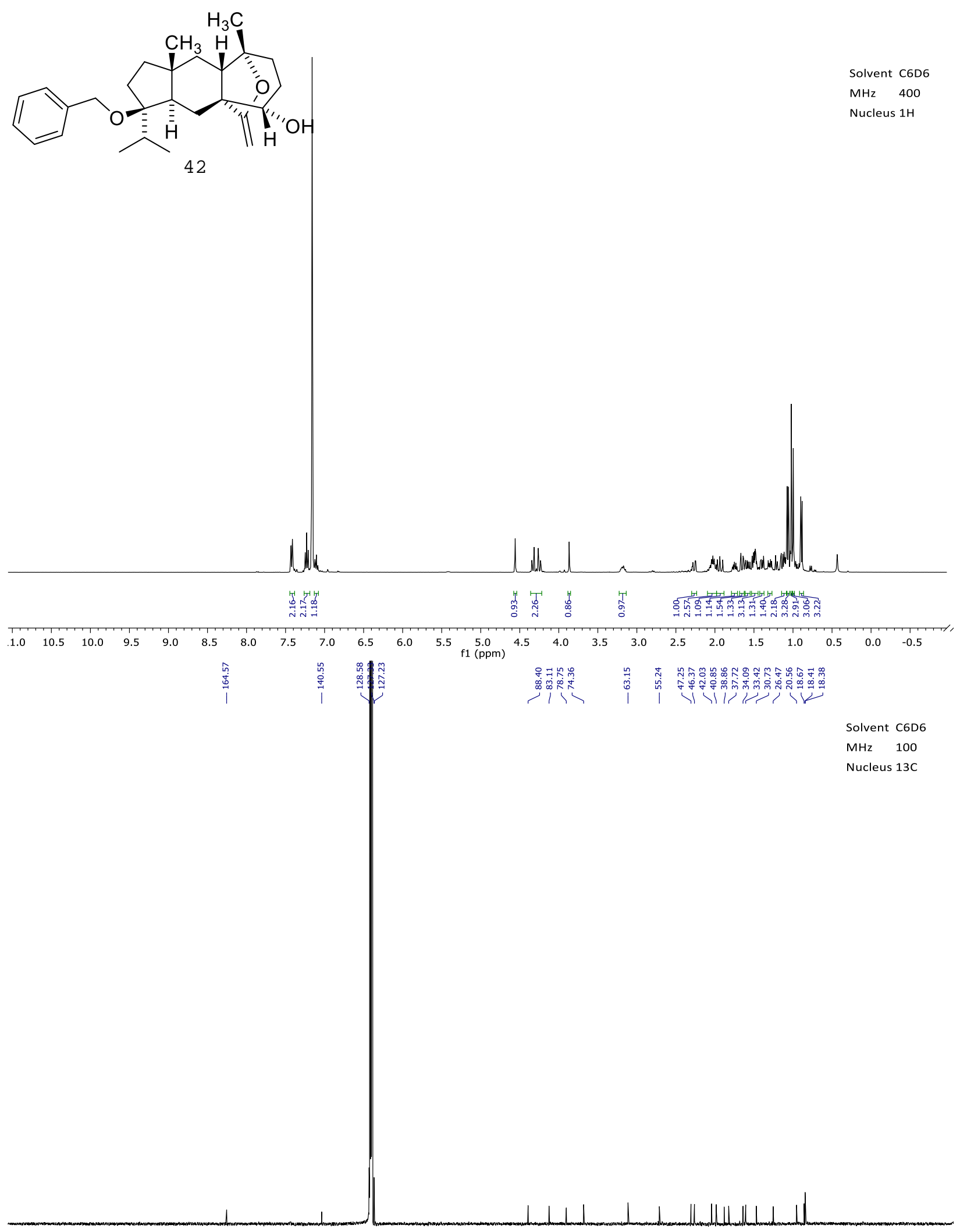

$\begin{array}{llllllllllllllllllllllll} & 210 & 200 & 190 & 180 & 170 & 160 & 150 & 140 & 130 & 120 & 110 & 100 & 90 & 80 & 70 & 60 & 50 & 40 & 30 & 20 & 10 & 0 & -10\end{array}$




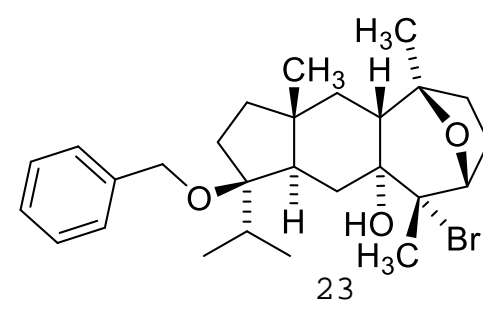

Solvent C6D6

$\mathrm{MHz} \quad 800$

Nucleus $1 \mathrm{H}$ 23

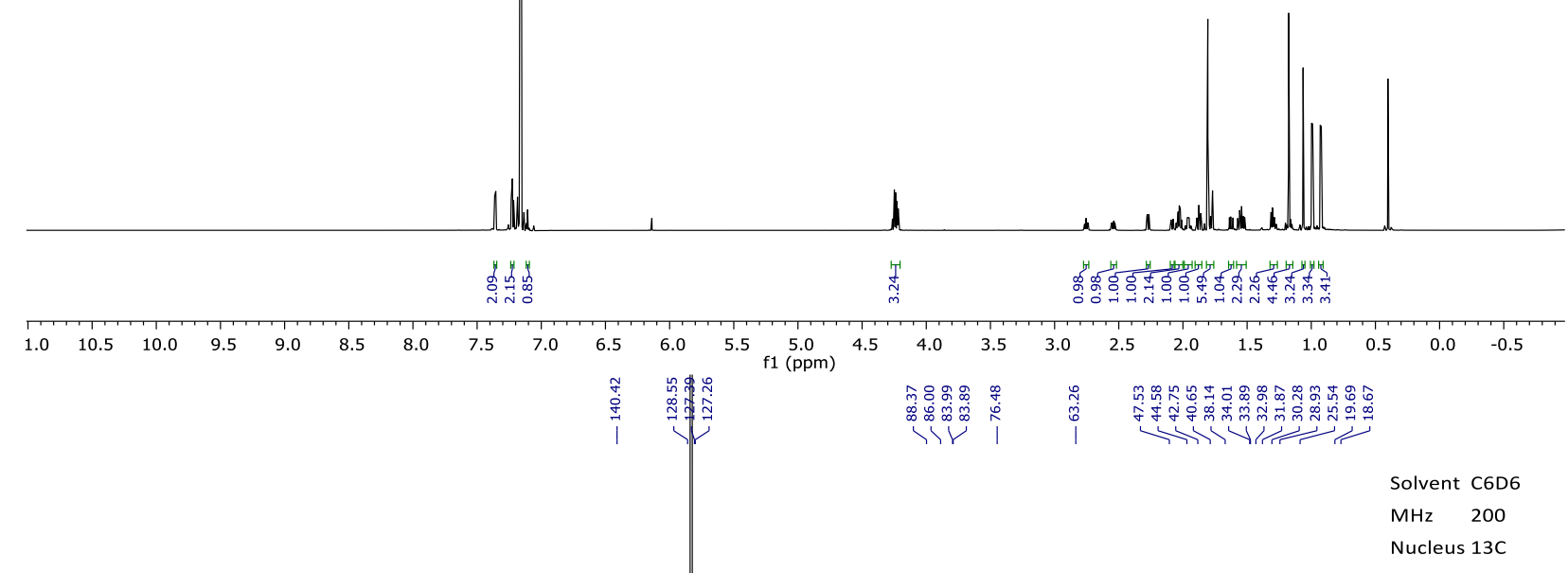

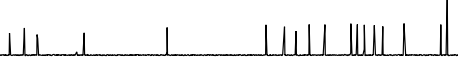




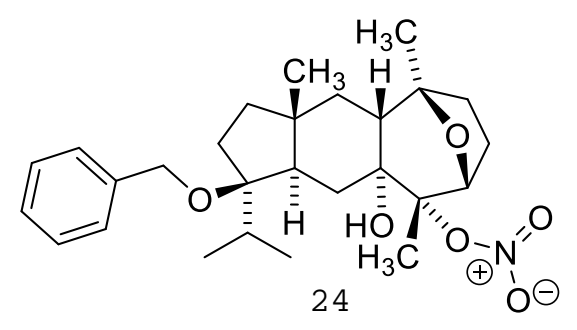

Solvent C6D6

$\mathrm{MHz} \quad 600$

Nucleus $1 \mathrm{H}$

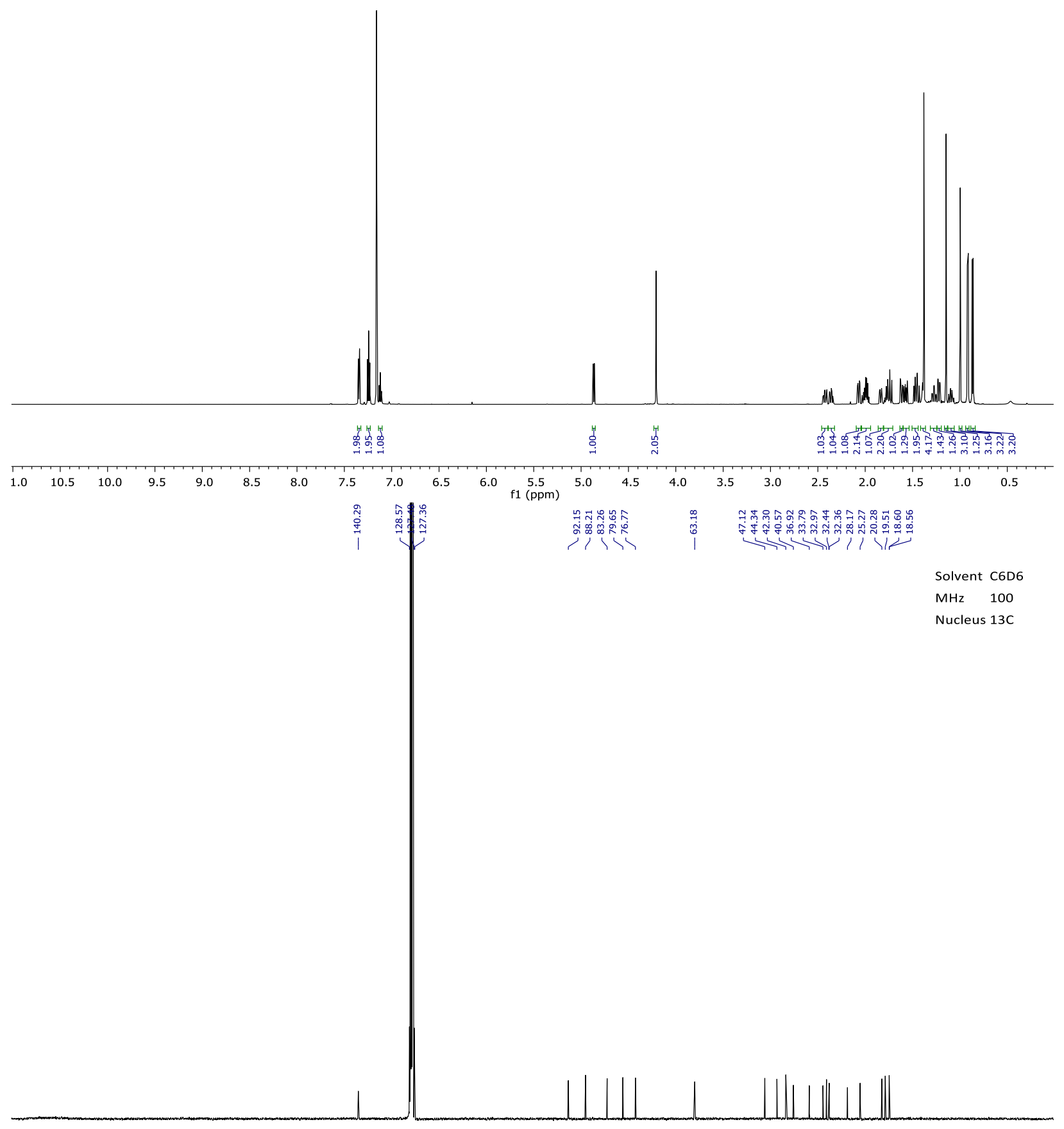

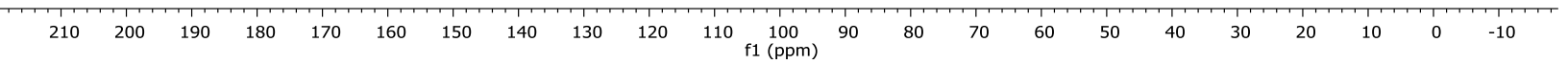




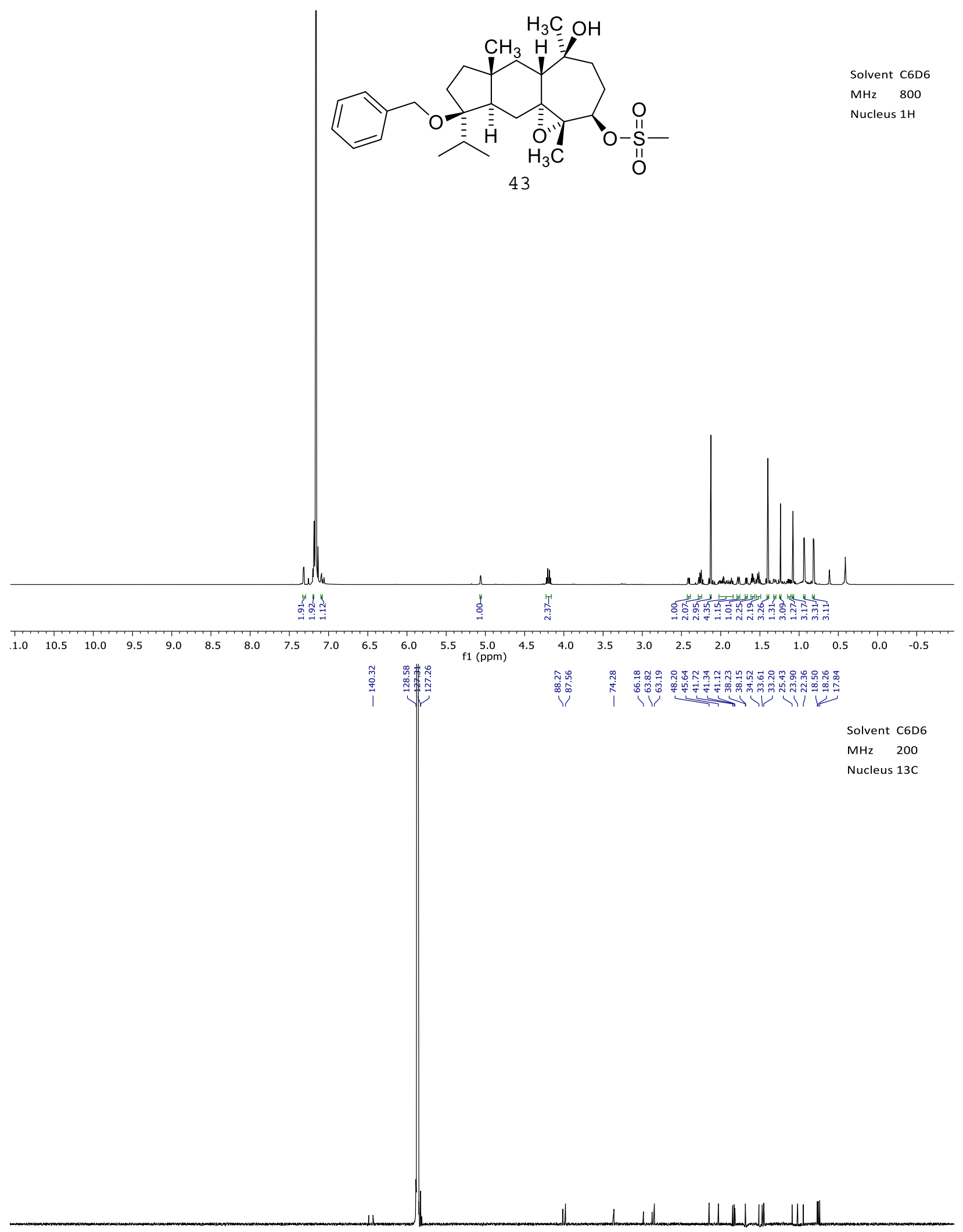

$\begin{array}{lllllllllllllllllllllllllllll}230 & 220 & 210 & 200 & 190 & 180 & 170 & 160 & 150 & 140 & 130 & 120 & 110 & 100 & 90 & 80 & 70 & 60 & 50 & 40 & 30 & 20 & 10 & 0 & -10\end{array}$



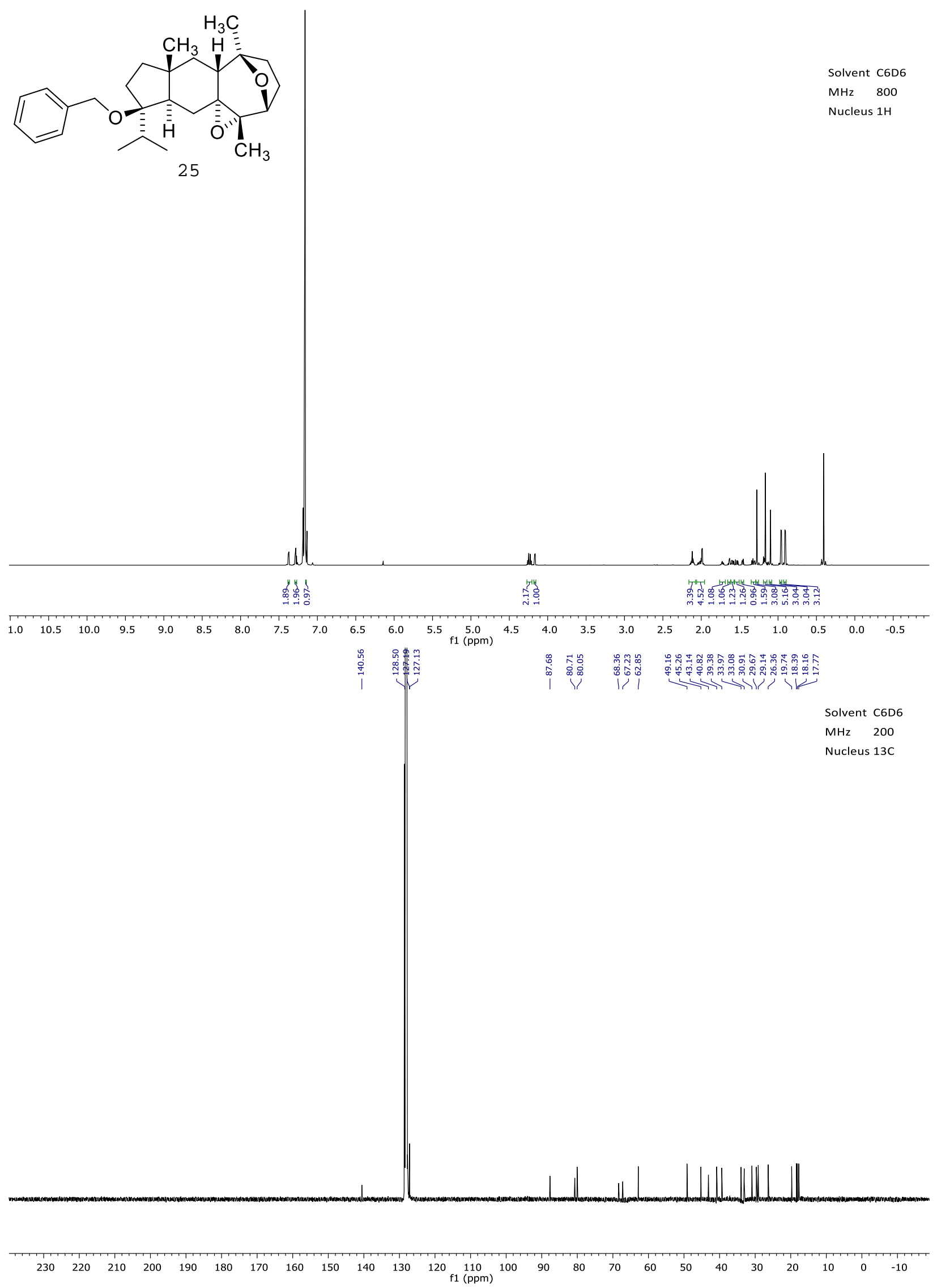
<smiles>CC(=O)C12C[C@]3(C)CC[C@](O)(C(C)C)[C@@]3(C)C[C@H]1[C@@H]1CC[C@]2(C)O1</smiles>

Solvent C6D6
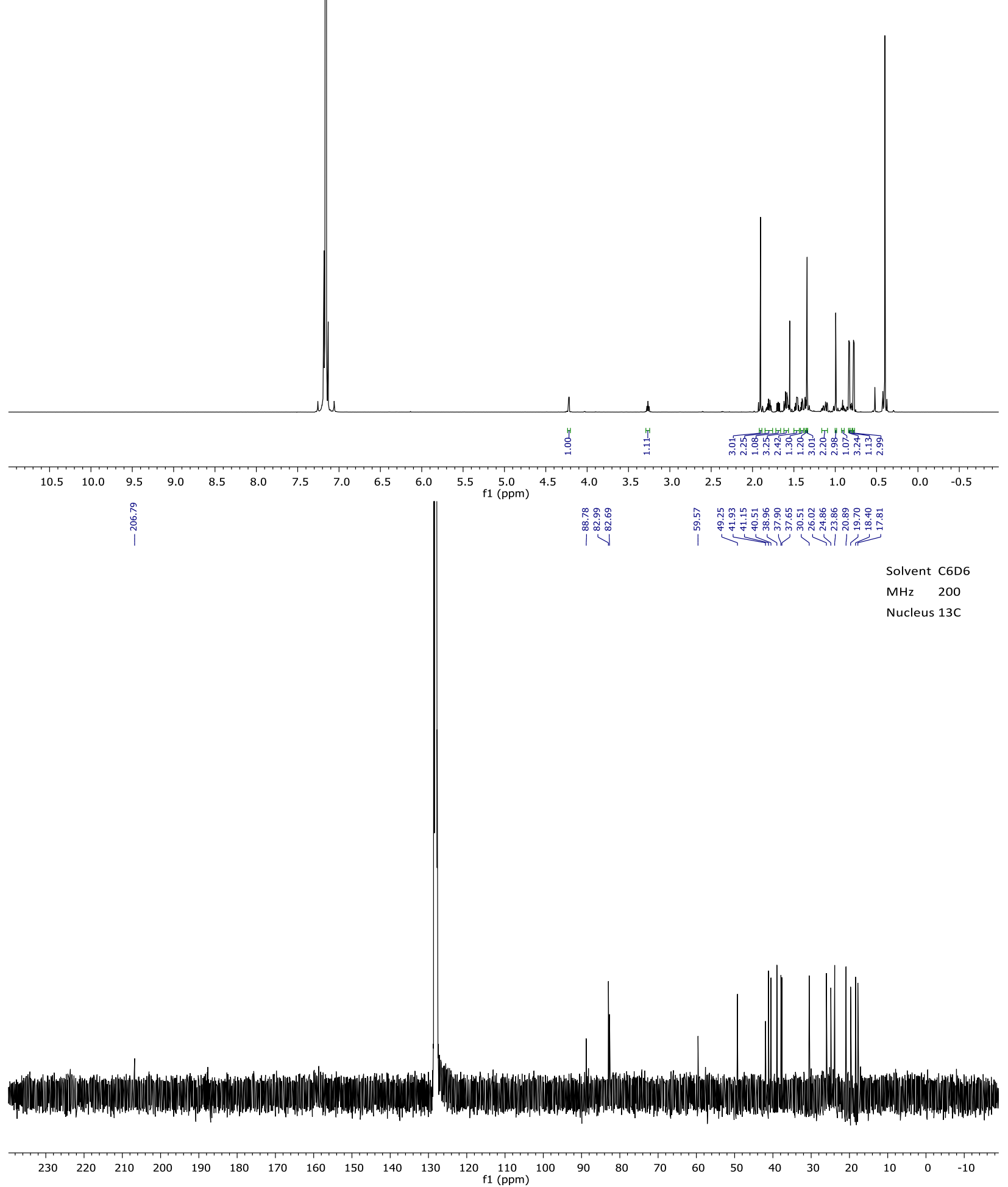


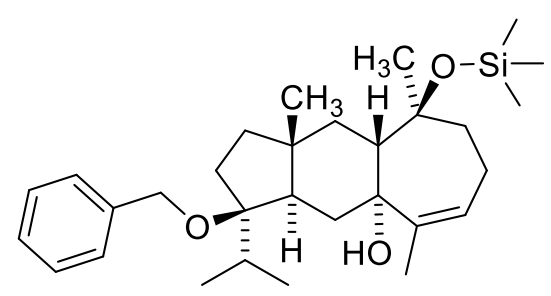

Solvent C6D6

$\mathrm{MHz} \quad 400$

Nucleus $1 \mathrm{H}$

44

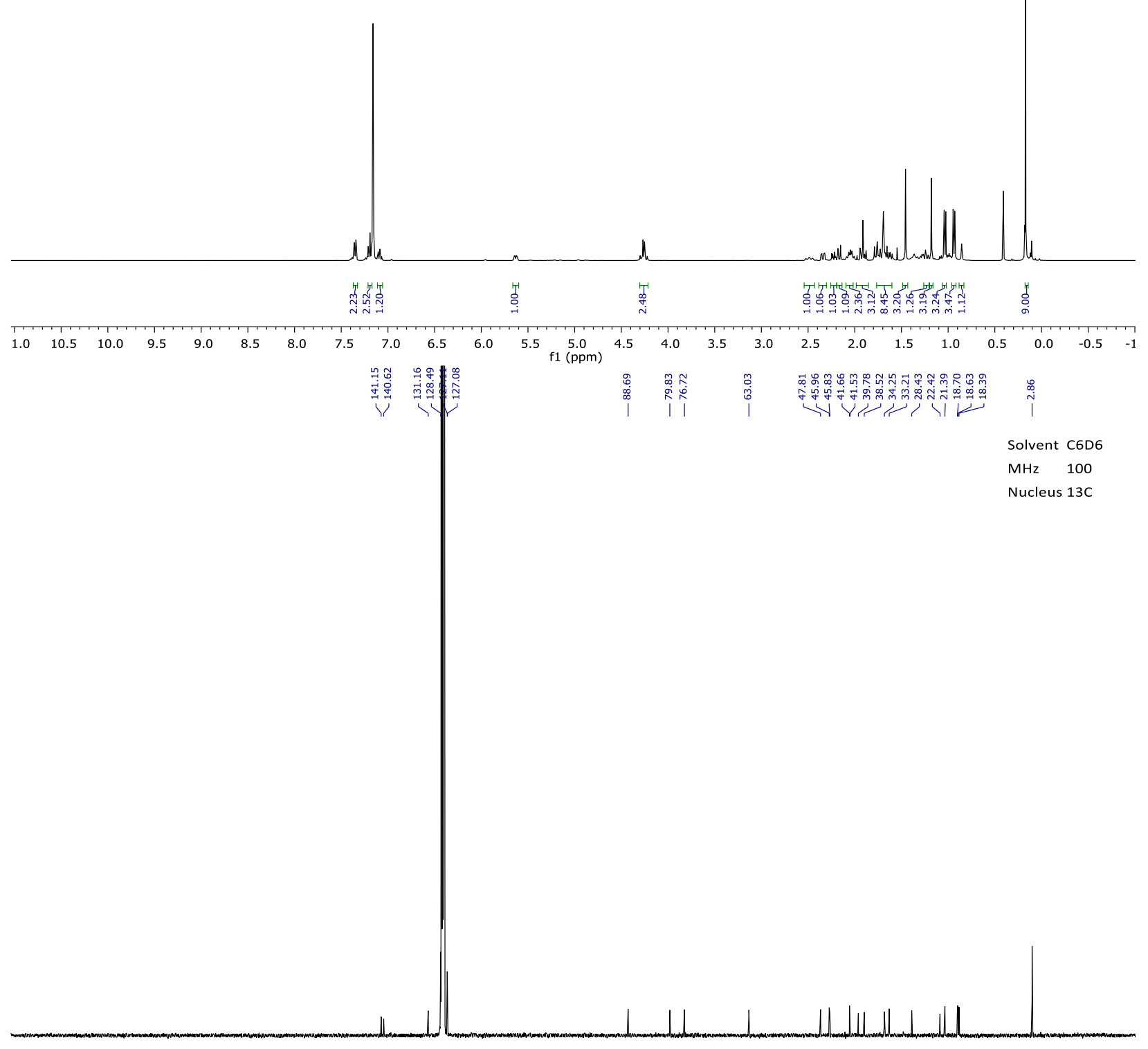

$\begin{array}{llllllllllllllllllllllll}210 & 200 & 190 & 180 & 170 & 160 & 150 & 140 & 130 & 120 & 110 & 100 & 90 & 80 & 70 & 60 & 50 & 40 & 30 & 20 & 10 & 0 & -10\end{array}$




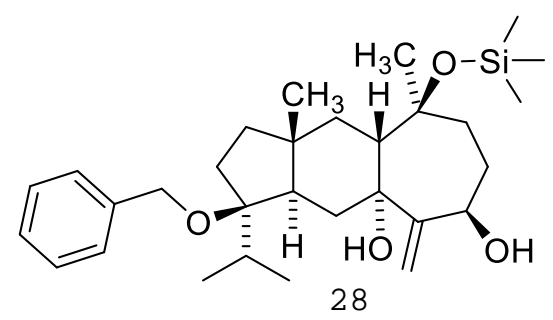

Solvent C6D6

$\mathrm{MHz} \quad 800$

Nucleus $1 \mathrm{H}$

28

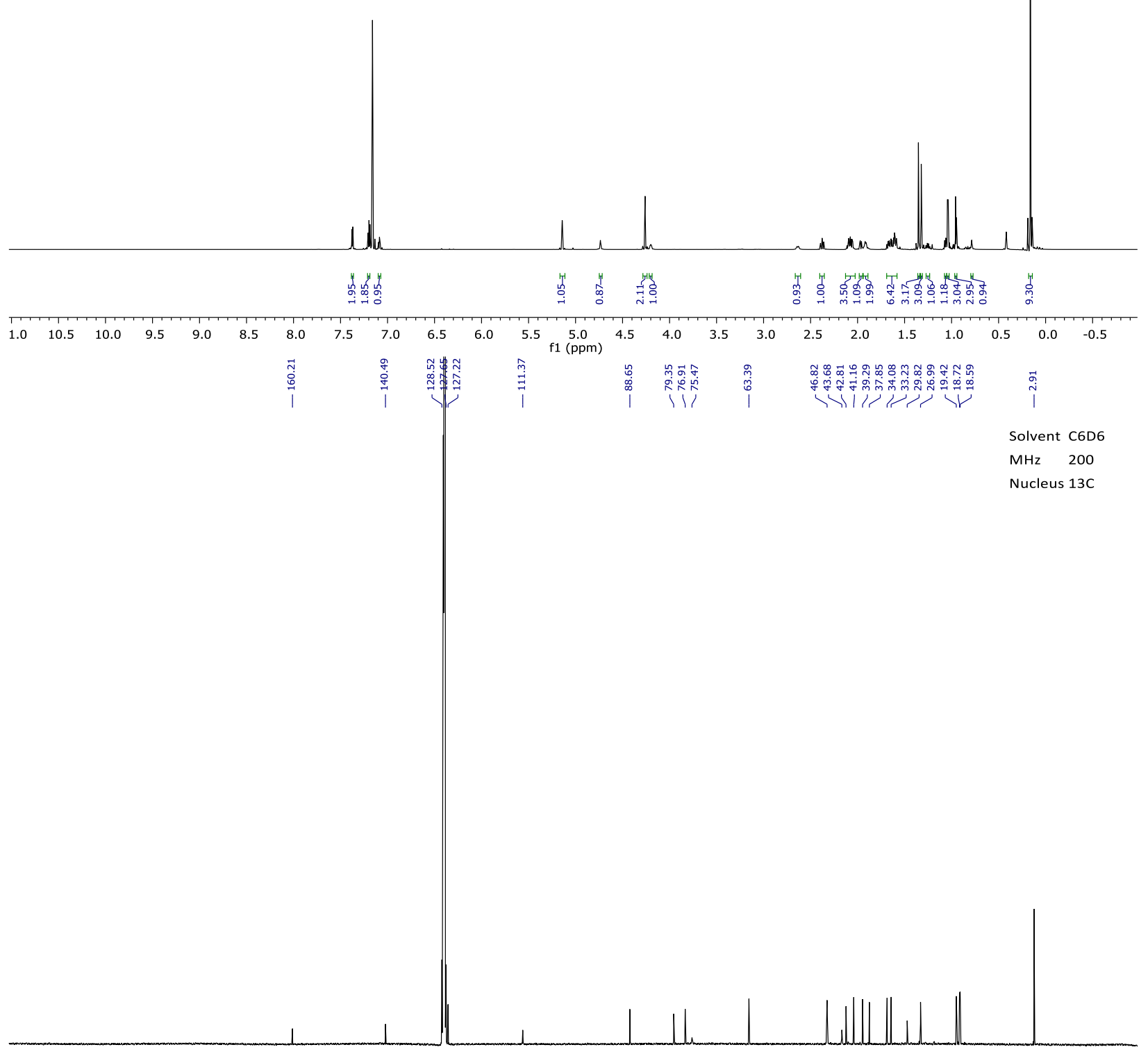

\begin{tabular}{llllllllllllllllllllllllllll}
\hline 20 & 210 & 200 & 190 & 180 & 170 & 160 & 150 & 140 & 130 & 120 & 110 & 100 & 90 & 80 & 70 & 60 & 50 & 40 & 30 & 20 & 10 & 0 & -10
\end{tabular} 


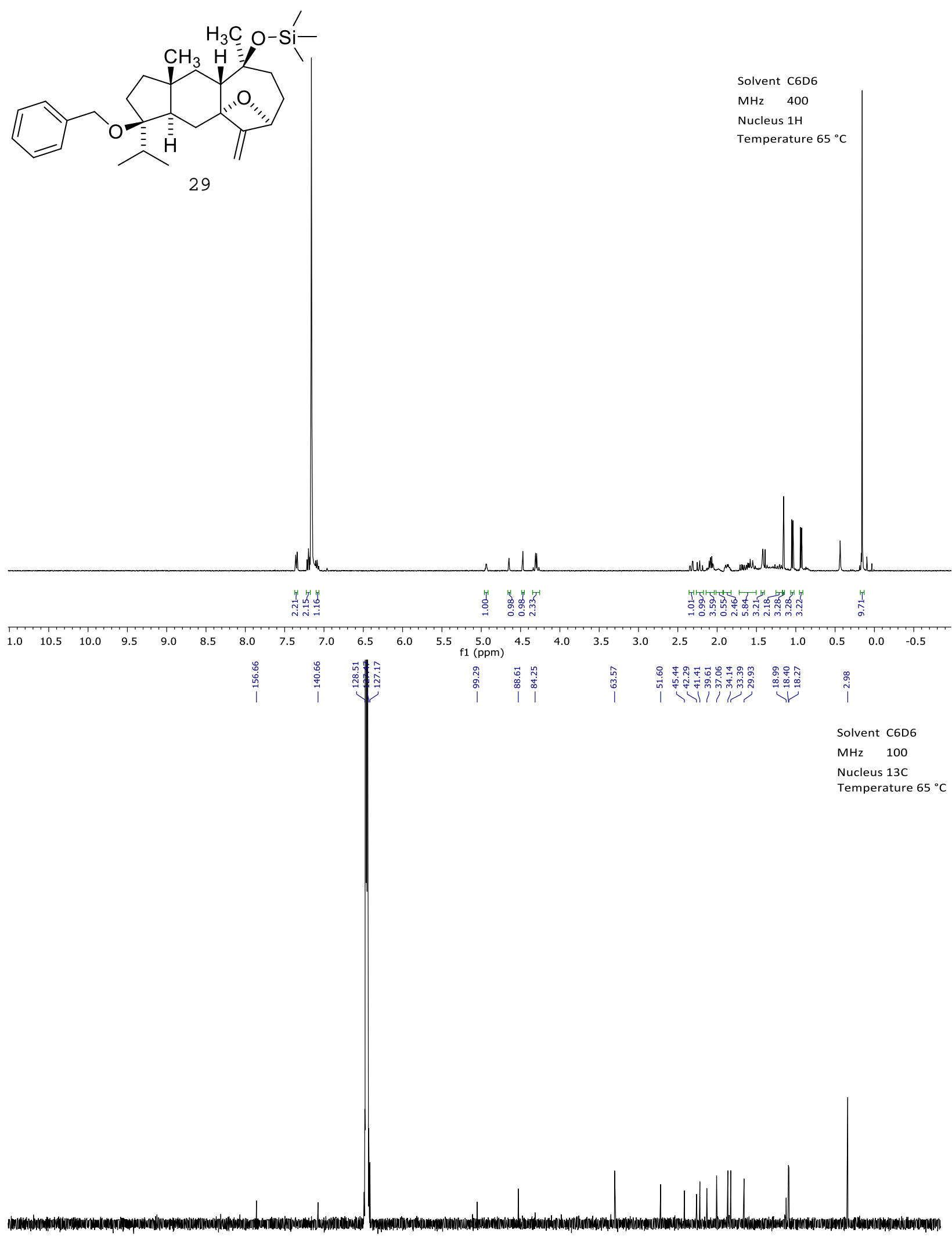

$\begin{array}{lllllllllllllllllllllllllll}220 & 210 & 200 & 190 & 180 & 170 & 160 & 150 & 140 & 130 & 120 & 110 & 100 & 90 & 80 & 70 & 60 & 50 & 40 & 30 & 20 & 10 & 0 & -10 & -20\end{array}$ 

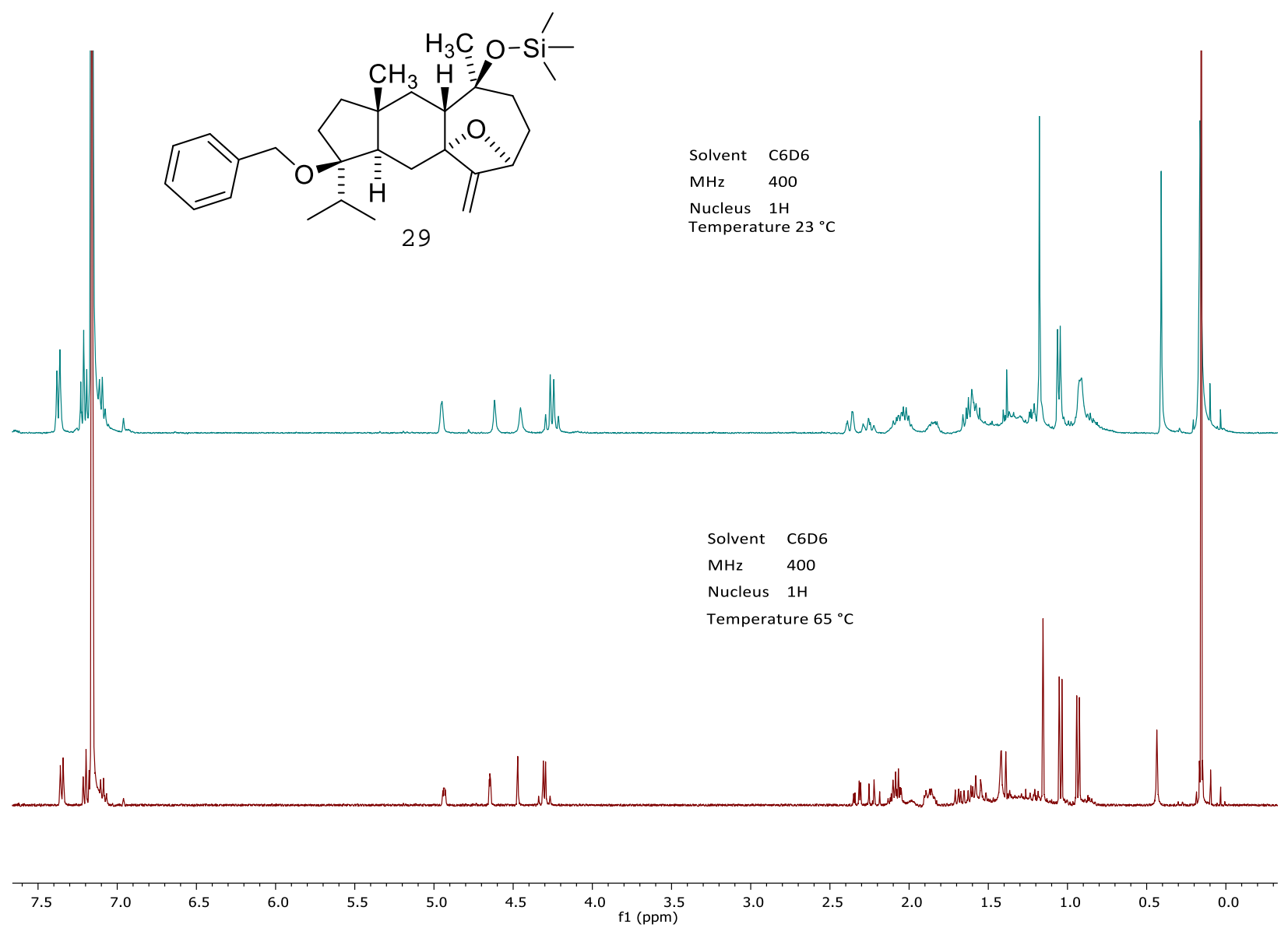


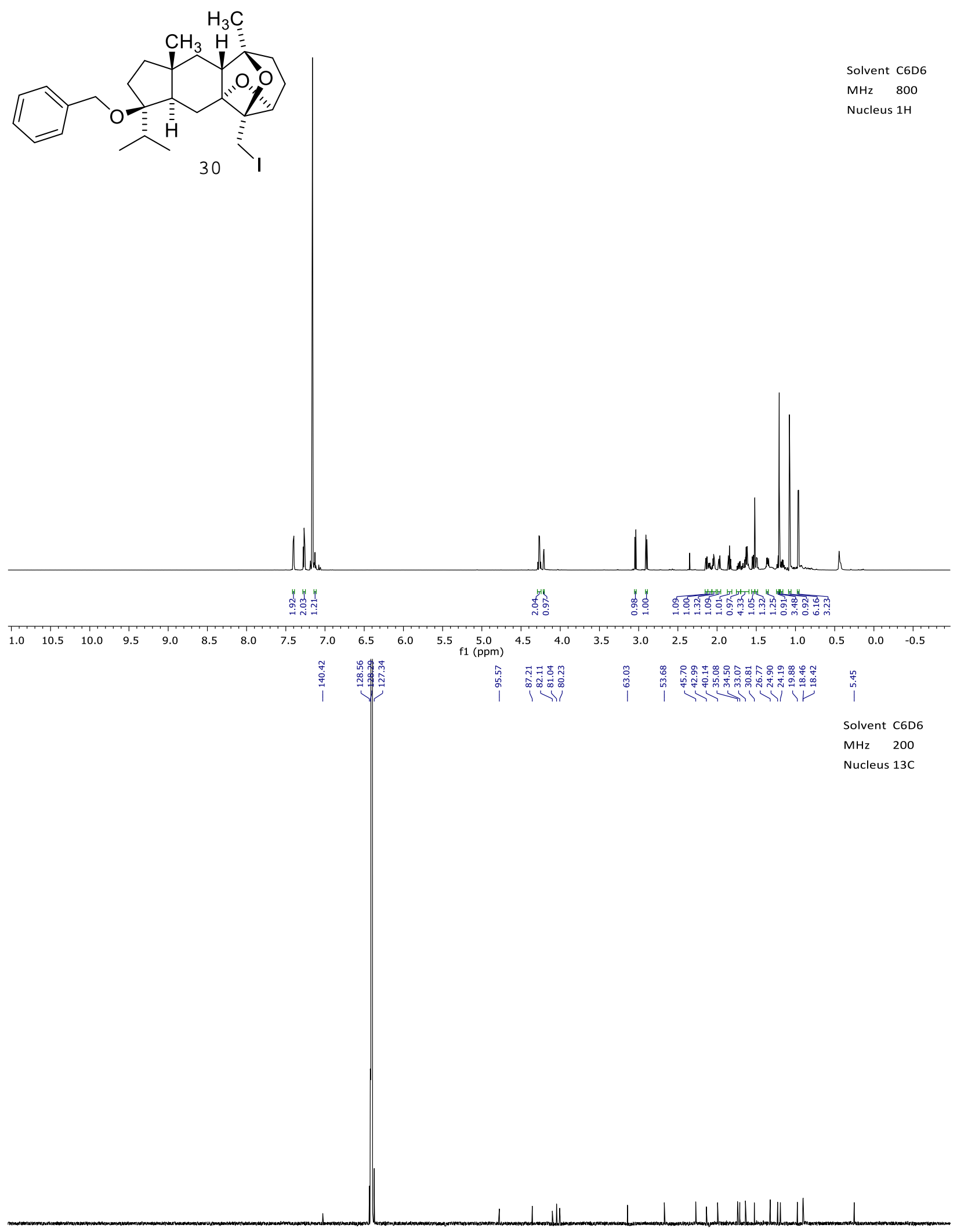

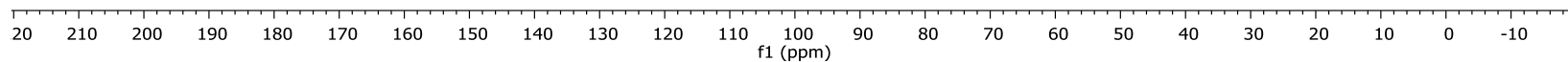




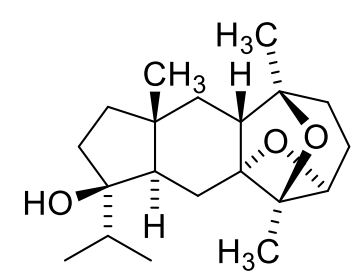

Solvent $\mathrm{CDCl} 3$

$\mathrm{MHz} \quad 800$

Nucleus $1 \mathrm{H}$

dictyoxetane (1)

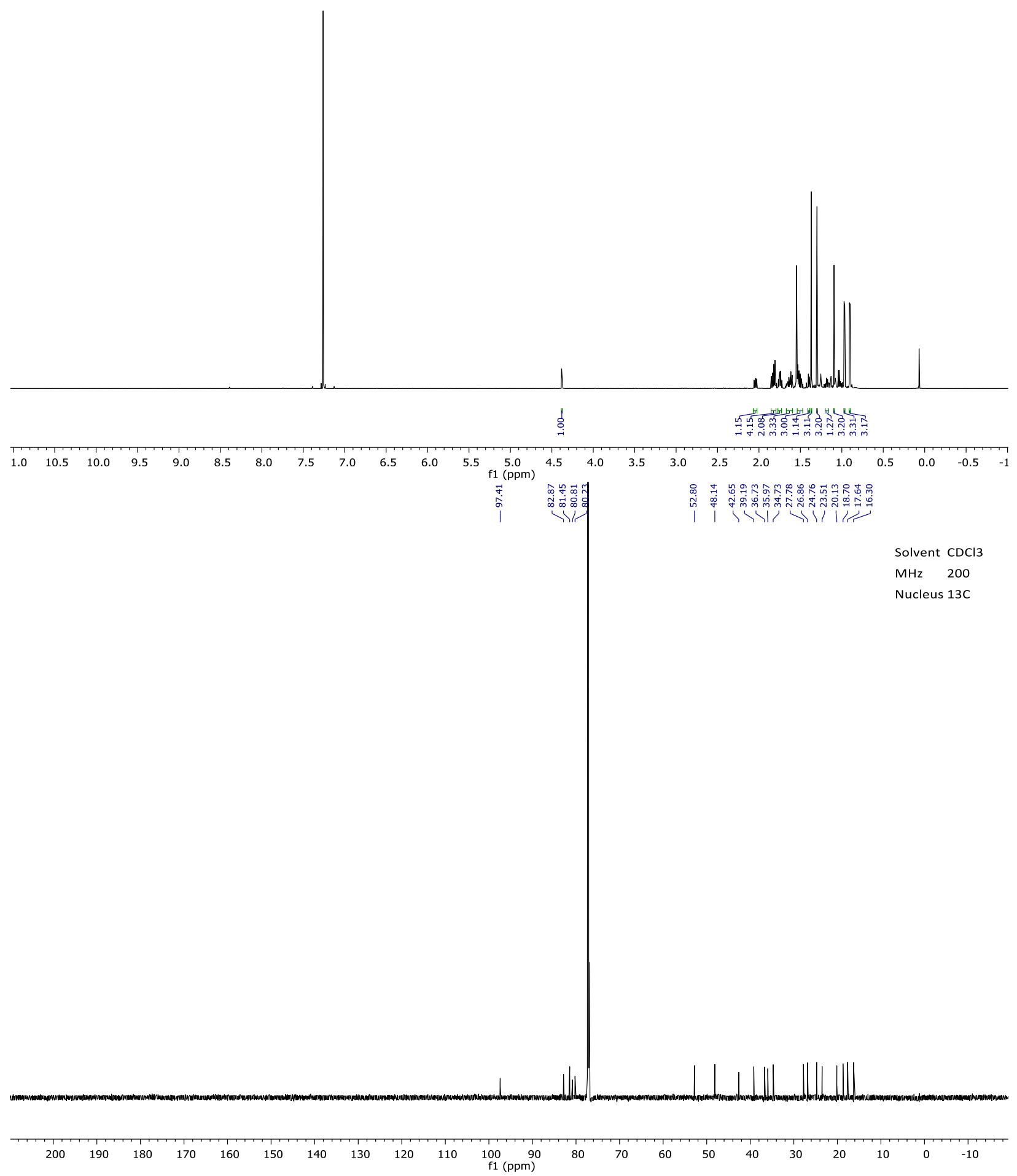




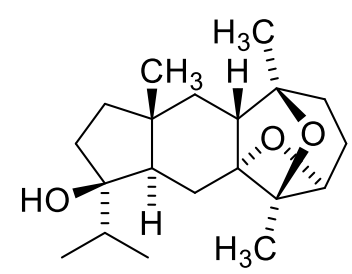

dictyoxetane (1)

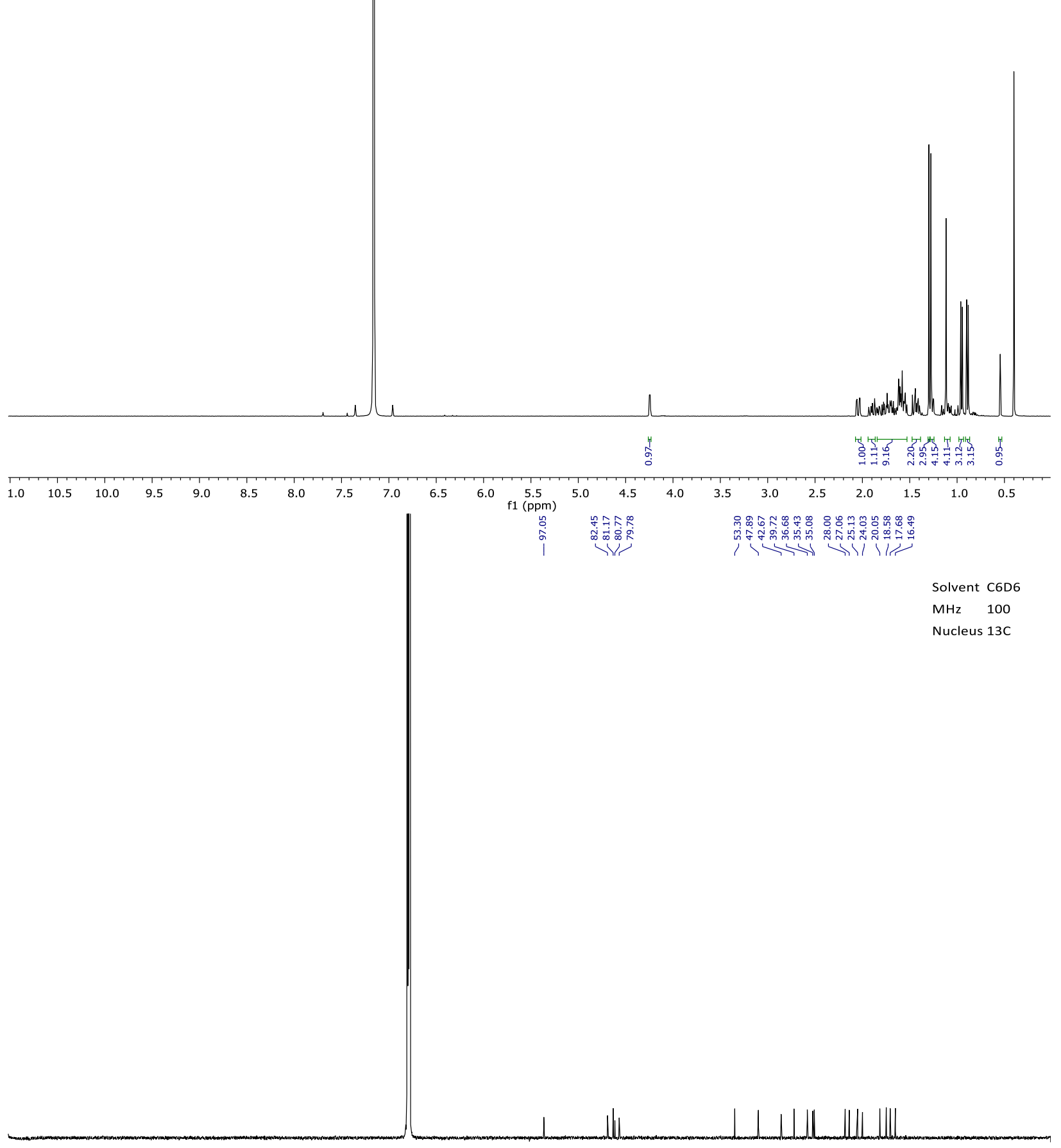

$\begin{array}{lllllllllllllllllllllllllllll}210 & 200 & 190 & 180 & 170 & 160 & 150 & 140 & 130 & 120 & 110 & 100 & 100 & 80 & 70 & 60 & 50 & 40 & 30 & 20 & 10 & 0 & -10\end{array}$




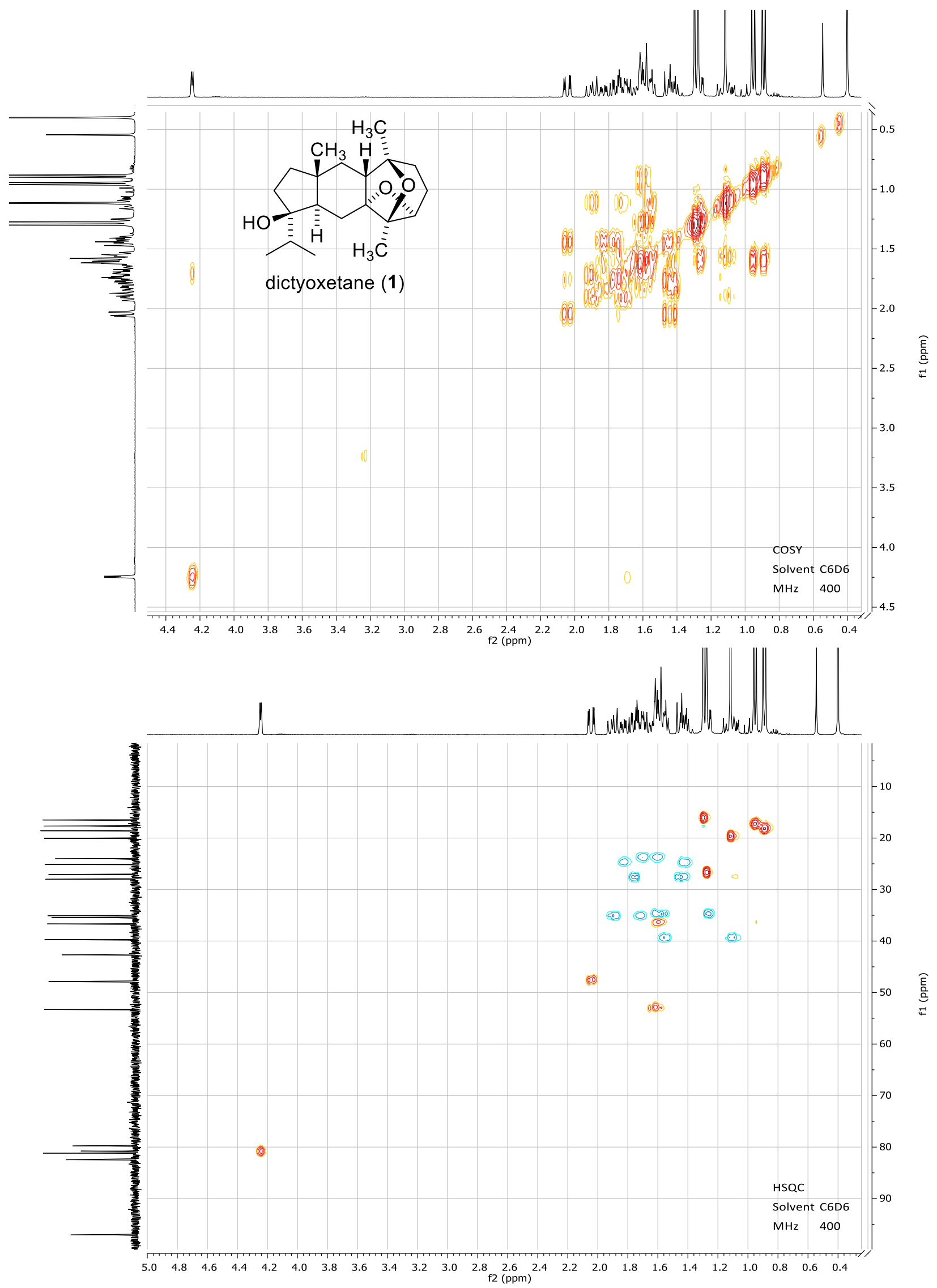



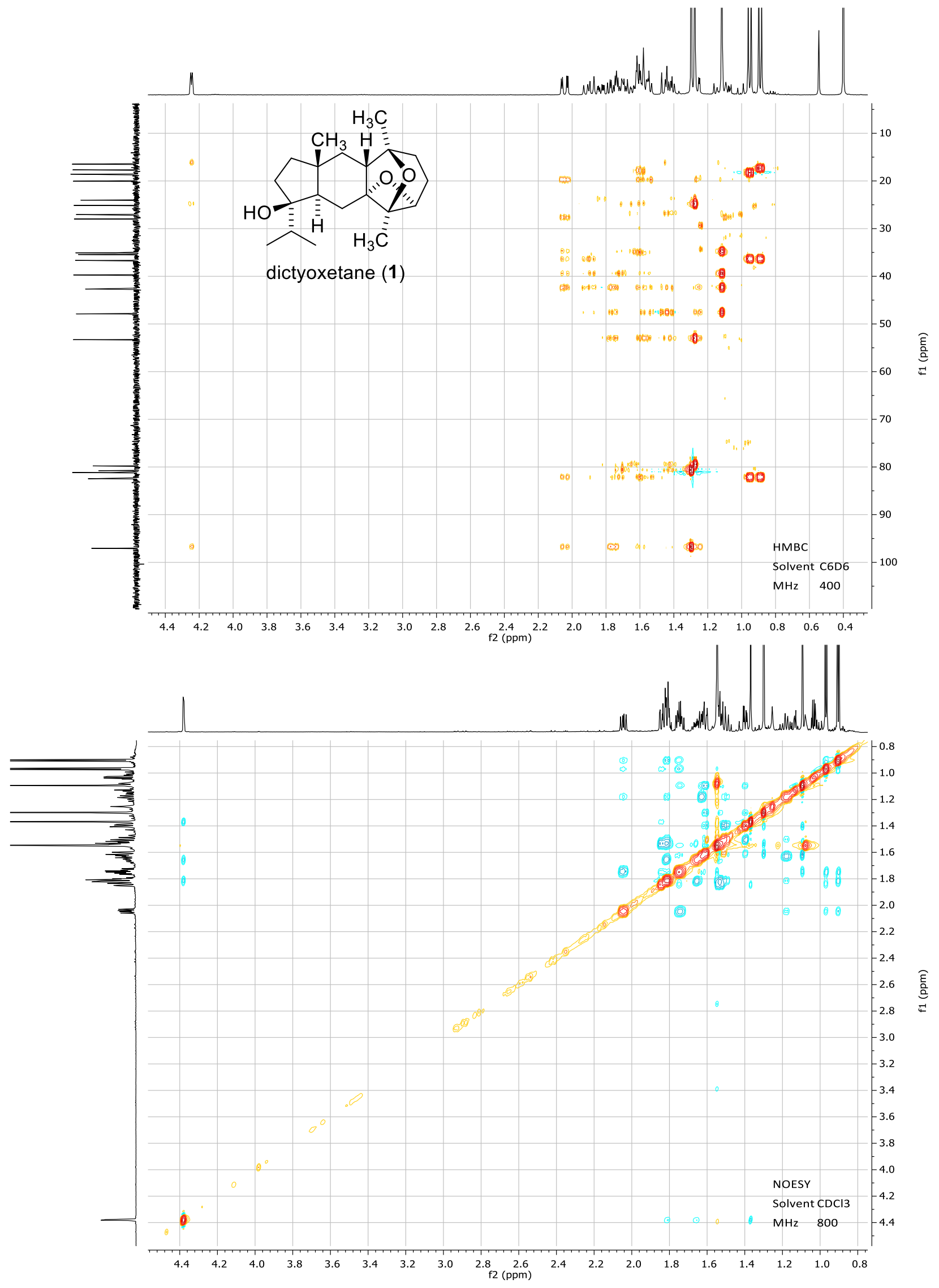
${ }^{1}$ W.C. Still, M. Kahn, A. J. Mitra, J. Org. Chem. 1978, 43, 2923.

${ }^{2}$ W. G. Kofron, L. M. Baclawski, J. Org. Chem. 1976, 41, 1879.

${ }^{3}$ Prepared according to: D. F. Taber, P. W. Dematteo, R. A. Hassan, Org. Synth. 2013, 90, 350.

4 a) M. Pfau, G. Revial, A. Guingant, J. d'Angelo, J. Am. Chem. Soc. 1985, 107, 273. b) G. Revial, M. Pfau, Org. Synth. $1992,70,35$.

${ }^{5}$ E. Canales, E. J. Corey, J. Am. Chem. Soc. 2007, 129, 12686.

${ }^{6}$ a) D. Becker, N. C. Brodsky, J. Kalo, J. Org. Chem. 1978, 43, 2557. b) D. Becker, J. Kalo, N. C. Brodsky, J. Org. Chem. 1978, 43, 2562.

${ }^{7}$ B. Defaut, T. B. Parsons, N. Spencer, L. Male, B. M. Kariuki, R. S. Grainger, Org. Biomol. Chem. 2012, $10,4926$.

${ }^{8}$ Epoxidation of tricycle 14 with $m$-CPBA $\left(\mathrm{CH}_{2} \mathrm{Cl}_{2},{ }^{\circ} \mathrm{C}\right)$ gave an unfavourable 2:3 mixture of epoxide 15 and tetrahydrofuran 39.

${ }^{9}$ This intermediate could also be obtained via the dyotropic rearrangement of epoxide-oxetane 22 , see " 2.20 Synthesis of dioxatricycle 17" for characterization of this product.

${ }^{10}$ Reaction monitoring by TLC analysis (30\% ethyl acetate in hexanes) showed gradual conversion of epoxide $15\left(R_{f}=0.15\right)$ to a putative bromo-triol intermediate $\left(R_{f}=0.30\right)$, which then reacted further to bromohydrin $\mathbf{2 0}\left(R_{f}=0.80\right)$.

${ }^{11}$ Reaction monitoring by TLC analysis (30\% ethyl acetate in hexanes) showed gradual conversion of iodide $\mathbf{3 0}\left(R_{f}=0.77\right)$ to the dehalogenated intermediate $\left(R_{f}=0.69\right)$ (usually within $\left.1-2 \mathrm{~h}\right)$, which then was further reduced to $(+)$-dictyoxetane $(1)\left(R_{f}=0.34\right)$. Occasionally the reaction was found to stall after complete dehalogenation. In these cases, the mixture was filtered through a pad of celite and the filtrate was concentrated. The residue was then resubmitted to the analogous hydrogenation conditions with fresh palladium on carbon.

12 a) K. C. Pullaiah, R. K. Surapaneni, C. B. Rao, K. F. Albizati, B. W. Sullivan, D. J. Faulkner, H. Cun-heng, J. Clardy, J. Org. Chem. 1985, 50, 3665. b) C. B. Rao, K. C. Pullaiah, R. K. Surapaneni, B. W. Sullivan, K. F. Albizati, D. J. Faulkner, H. Cunheng, J. Clardy, J. Org. Chem. 1986, 51, 2736.

${ }^{13}$ A. Altomare, M. C. Burla, M. Camalli, G. L. Cascarano, C. Giacovazzo, A. Guagliardi, A. G. G. Moliterni, G. Polidori, R. Spagna, J. Appl. Crystallogr. 1999, 32, 115.

${ }^{14}$ G. M. Sheldrick, Acta Crystallogr. 2008, A64, 112. 